\title{
Combination and integration to redirect NK cells for cancer immunotherapy
}

Citation for published version (APA):

Gong, Y. (2021). Combination and integration to redirect NK cells for cancer immunotherapy. [Doctoral Thesis, Maastricht University]. Maastricht University. https://doi.org/10.26481/dis.20211130yg

Document status and date:

Published: 01/01/2021

DOI:

10.26481/dis.20211130yg

Document Version:

Publisher's PDF, also known as Version of record

\section{Please check the document version of this publication:}

- A submitted manuscript is the version of the article upon submission and before peer-review. There can be important differences between the submitted version and the official published version of record.

People interested in the research are advised to contact the author for the final version of the publication, or visit the DOI to the publisher's website.

- The final author version and the galley proof are versions of the publication after peer review.

- The final published version features the final layout of the paper including the volume, issue and page numbers.

Link to publication

\footnotetext{
General rights rights.

- You may freely distribute the URL identifying the publication in the public portal. please follow below link for the End User Agreement:

www.umlib.nl/taverne-license

Take down policy

If you believe that this document breaches copyright please contact us at:

repository@maastrichtuniversity.nl

providing details and we will investigate your claim.
}

Copyright and moral rights for the publications made accessible in the public portal are retained by the authors and/or other copyright owners and it is a condition of accessing publications that users recognise and abide by the legal requirements associated with these

- Users may download and print one copy of any publication from the public portal for the purpose of private study or research.

- You may not further distribute the material or use it for any profit-making activity or commercial gain

If the publication is distributed under the terms of Article $25 \mathrm{fa}$ of the Dutch Copyright Act, indicated by the "Taverne" license above, 
Combination and Integration to Redirect NK cells for Cancer Immunotherapy 


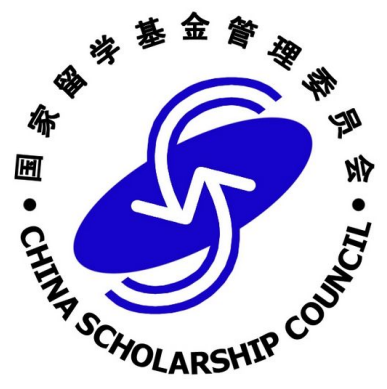

Maastricht University

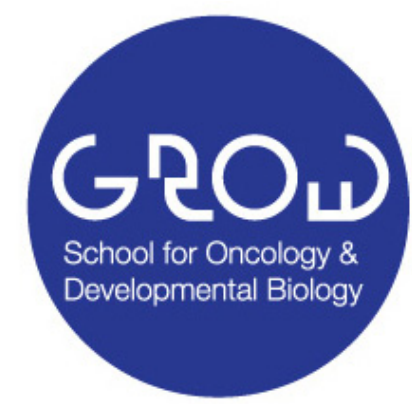

Maastricht UMC+

(c) Ying Gong, Maastricht 2021

All rights are reserved. For articles published, the copyright has been transferred to the respective publisher. No parts of this thesis may be reproduced, stored in a retrieval system or transmitted in any form or by any means, without prior permission from the author.

Cover design: Ying Gong

Layout: $\quad$ Ying Gong

Printed by: $\quad$ Ridderprint | www.ridderprint.nl.

ISBN: $\quad$ 978-94-6416-830-3 


\section{Combination and Integration to Redirect NK cells for Cancer Immunotherapy}

\section{DISSERTATION}

to obtain the degree of Doctor at Maastricht University, on the authority of the Rector Magnificus,

Prof. dr. Rianne M. Letschert, in accordance with the decision of the Board of Deans, to be defended in public on Tuesday 30 November 2021, at 13:00 hours

by

\section{Ying Gong}

\section{龚英}

Born on 06-July-1989

In Ganzhou, P.R. China 


\section{Supervisors}

Dr. Wilfred T.V. Germeraad

Prof. dr. Gerard M.J. Bos

\section{Co-supervisor}

Dr. Roel G.J. Klein Wolterink

\section{Assessment Committee}

Prof. dr. Dirk De Ruysscher (Chairman) (MAASTRO Clinic, Maastricht University

Medical Center+)

Prof. dr. Erik Beckers (Division of Hematology, Department of Internal Medicine, Maastricht University Medical Center+)

Dr. Ludwig Dubois (Department of Precision Medicine, GROW - School for Oncology, Maastricht University)

Prof. dr. Jürgen Kuball (Department of Medical Oncology, University Medical Center Utrecht)

Prof. dr. Tuna Mutis (Cancer Center Amsterdam, VU University Medical Center, Amsterdam UMC)

The research presented in this thesis was conducted at the Laboratory of Hematology/ Tumor immunology, Department of Internal Medicine, GROW - School for Oncology and Developmental Biology, Maastricht University, Maastricht, The Netherlands.

I thank the China Scholarship Council (CSC) (No. 201707720056) for fellowship support. Financial support by the Cancer Research Foundation Limburg (KOFL-2012-03) and GROW School of Maastricht University for the publication of this thesis is gratefully acknowledged. 


\section{Contents}

\section{Abbreviations}

Chapter 1 General Introduction

Chapter 2 Defucosylation of tumor-specific humanized anti-MUC1 monoclonal antibody enhances NK cell-mediated anti-tumor cell cytotoxicity

Chapter 3 Rosuvastatin enhances VSV-G lentiviral transduction of Natural Killer cells via upregulation of the Low-Density Lipoprotein receptor

Chapter 4 Chimeric Antigen Receptor Natural Killer (CAR-NK) cell design and engineering for cancer therapy

Chapter 5 NKG2A genetic deletion promotes human NK cell anti-tumor responses by reversing HLA-E-mediated inhibition

Chapter 6 General Discussion

Chapter 7 Summary 



\section{Abbreviations}

ACT

ADAM17

ADCC

ADCP

ADEPT

ALL

AML

APC

ATMP

BCMA

CAR

Cas9

CCR

CD

CDC

CLL

CML

CR

CRS

CRISPR

DLBCL

EGFR

EMA

FACS

FcyRIII

Fas L
Adoptive cellular therapy

ADAM Metallopeptidase Domain 17

Antibody-dependent cellular cytotoxicity

Antibody-dependent cellular phagocytosis

Antibody-directed enzyme prodrug therapy

Acute lymphoblastic leukemia

Acute myeloid leukemia

Antigen-presenting cell

Advanced therapeutic medicinal product

$B$ cell maturation antigen

Chimeric antigen receptor

CRISPR-associated system 9

CC chemokine receptor

Cluster of differentiation

Complement-dependent cytotoxicity

Chronic lymphocytic leukemia

Chronic myeloid leukaemia

Complete remission

Cytokine release syndrome

Clustered regularly interspaced short palindromic repeats

Diffuse large B cell lymphoma

Epidermal growth factor receptor

European Medicines Agency's

Fluorescence-Activated Cell Sorting

Fc gamma receptor type III (CD16)

Fas ligand 
FDA

GalNAc

GGPP

GM-CSF

GVHD

Her2

hESC

HLA

HSPC

HSCT

ICANS

IFN-Y

IL-21

iPSC

IS

ITAM

ITIM

KIR

LDLR

MACS

MDS

METC

$\mathrm{MHC}$

MoAb

MM

$\mathrm{MOI}$

MUC1

MUC1-Tn

MUC1-STn
Food and Drug Administration

$\mathrm{N}$-Acetylgalactoseamine

Geranylgeranyl-pyrophosphate

Granulocyte-macrophage colony-stimulating factor

Graft-versus-host disease

Human epidermal growth factor receptor 2

human embryonic stem cell

Human leukocyte antigen

Hematopoietic stem/progenitor cell

Hematopoietic stem cell transplantation

Immune cell-associated neurotoxicity syndrome

Interferon-y

Interleukin-21

Induced pluripotent stem cell

Immunologic synapse

Immunoreceptor tyrosine-based activation motif Immunoreceptor tyrosine-based inhibitory motif

Killer immuno-globulin receptor

Low density lipoprotein receptor

MACS ${ }^{\circledR}$ MicroBeads for column-based magnetic cell isolation

Myelodysplastic syndromes

Medical Ethical Review Committee

Major histocompatibility complex

Monoclonal antibody

Multiple myeloma

Multiplicity of infection

Mucin 1

MUC1 epitopes with carbohydrate antigens Tn

MUC1 epitopes with sialyl Tn (STn) antigens 


\begin{tabular}{ll} 
NCR & Natural cytotoxicity receptor \\
NK cell & Natural killer cell \\
NKG2A & Natural killer group 2 member A \\
NKG2D & Natural killer group 2 member D \\
NO & Nitric oxide \\
NSG mice & NOD SCID gamma mice \\
PB & Peripheral blood \\
PBMC & Peripheral Blood Mononuclear Cell \\
PBS & Phosphate-buffered saline \\
PD1 & Programmed cell death protein 1 \\
PGE2 & Prostaglandin E2 \\
PR & Partial remission \\
PSMA & Prostate-specific membrane antigen \\
PSCA & Prostate stem cell antigen \\
RAET1 & Retinoic acid early transcript 1 protein \\
RNPs & Ribonucleoprotein complexes \\
ROS & Reactive Oxygen Species \\
RT-PCR & Real-time PCR \\
sCFv & Single-chain antibody variable fragment \\
TAA & Tumor-associated antigen \\
TCR & T cell receptor \\
TGF- $\beta$ & Transforming growth factor-beta \\
TIGIT & T cell Immunoreceptor with lg and ITIM domains \\
TIM3 & T cell immunoglobulin and mucins domain-containing protein 3 \\
TME & Tumor microenvironment \\
TRAIL & TNF-related apoptosis-inducing ligand \\
TriKE & Tri-specific killer engager \\
UCB & Umbilical cord blood \\
VSV & Vesicular stomatitis virus \\
\hline
\end{tabular}





\section{Chapter 1}

General Introduction 
Cancer is one of the leading deadly diseases globally and requires great investigational efforts to understand the mechanism of tumorigenesis and find some more effective regimens to control it [1]. In 2020, the statistical data on global cancer diseases estimated about 19.3 million newly diagnosed cancer cases and 10 million cancer deaths [1]. Cancer cells are a group of heterogeneous cells derived from normal cells and express various atypical antigens [2]. Malignant cells show key hallmarks that include extensive growth potential, the ability to invade surrounding tissues and metastasize to distal organs [2,3]. Metastasis, a primary cause of mortality in cancer patients, is a complicated process and remains one of the least understood profiles of cancer biology [4].

Fortunately, scientists and physicians are engaged in developing novel therapeutic approaches to help patients suffering from cancer, and these approaches have achieved some breakthroughs and encouraging progress [5].

\section{Human Immune system}

Based on the speed and specificity of the immunoreaction, the immune system can be classified into two categories, including innate immunity and adaptive immunity [6].

The innate immune response is swift but sometimes causes injury to the normal healthy tissues since it is deficient in high specificity. Contrastingly, the adaptive response is accurate and takes several days to weeks to be generated [6,7]. The adaptive immune response can induce memory: consequent exposure with the same antigen a second time leads to a more rapid and robust response known as immune memory [8]. A brief overview of the classification of the immune system is shown in Figure 1.

Innate immunity includes physical barriers such as skin and mucosa, chemical, and microbiological barriers, as well as multiple immune cells, such as natural killer (NK) cells, monocytes, granulocytes, macrophages and immune mediators, such as the complement system and cytokines. Together, they provide immediate host defence towards pathogens or tumor cells [9]. In comparison, adaptive immunity is made up of antigen-specific B lymphocytes and T lymphocytes $[8,10]$. 


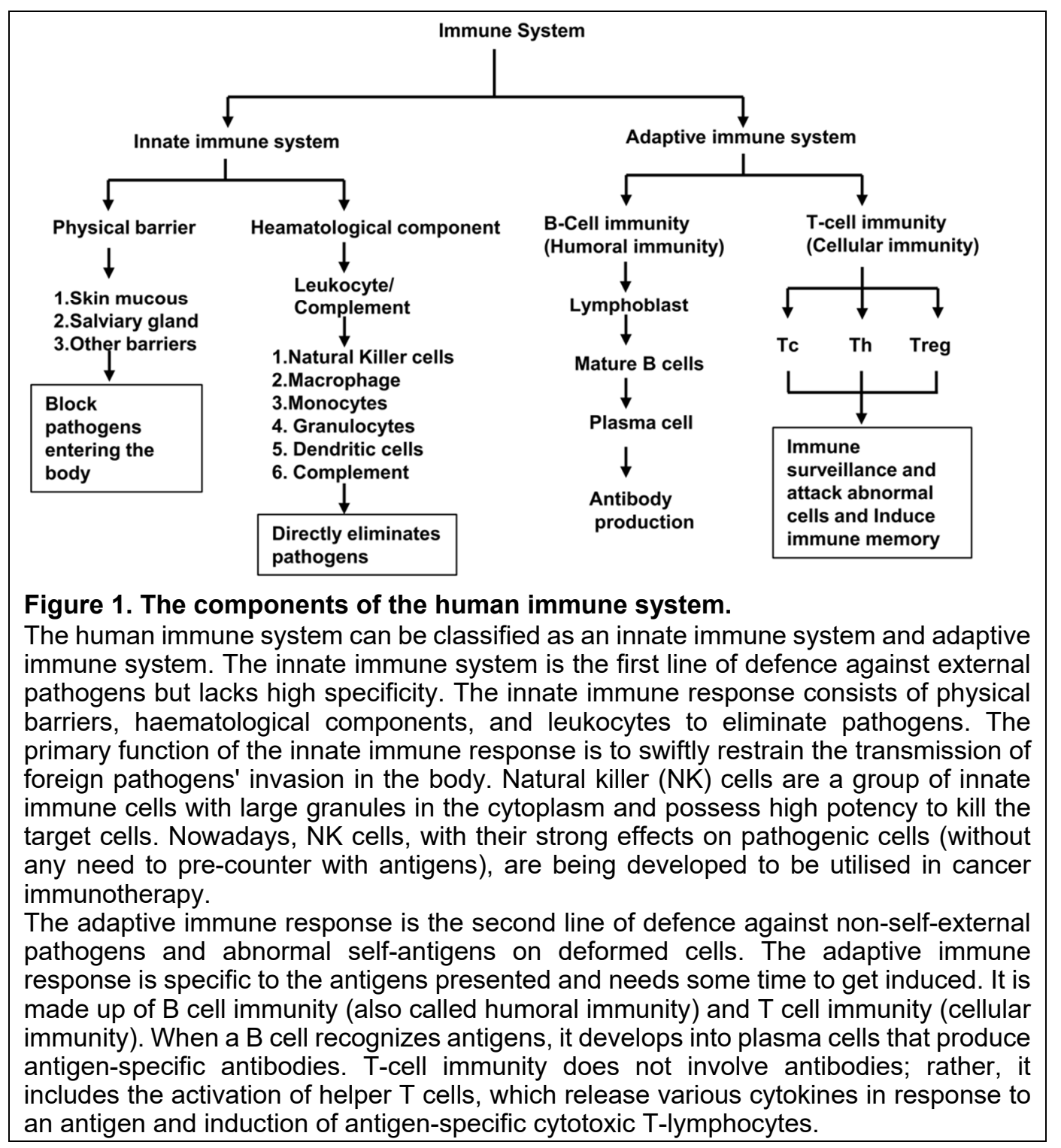

\section{Cancer immunotherapy}

Conventional treatments for tumors, like surgery, chemotherapy, and radiation, have been used for a long time in the clinical setting and have been considered to improve patients' survival and quality of life with malignant disease [11]. However, these conventional treatments possess one or more of the following limitations:

- Radio- and chemotherapy cannot precisely distinguish between normal and neoplastic cells; therefore they adversely also affect normal cells [12]. 
- Surgery and radiotherapy may not be suitable in patients with metastasis to the distal organs and multiple foci.

Therefore, there is an urgent need to develop novel cancer therapies that will target specific tumor cells.

In 2013, cancer immunotherapy has been considered a breakthrough of the year by the journal 'Science'. Immunotherapy holds the promise of high efficiency and specificity with minor to no effects on normal cells. Therefore, cancer immunotherapy has been broadly implicated in cancer treatment [13]. This method aims to boost the human immune system to eradicate tumor cells. The human immune system is a sophisticated network that protects the human body from detrimental risks, including external pathogens (such as bacteria, viruses, fungi, parasites, etc. derived from outside the body), abnormal or mutated endogenous cells (senescent cells, tumor cells and cell debris, originating from within the body), while protecting healthy cells and tissues from injury [6]. Figure 2 summarizes different approaches of for cancer immunotherapy, which have applied in the clinical setting. We will introduce some crucial methods for cancer immunotherapies.

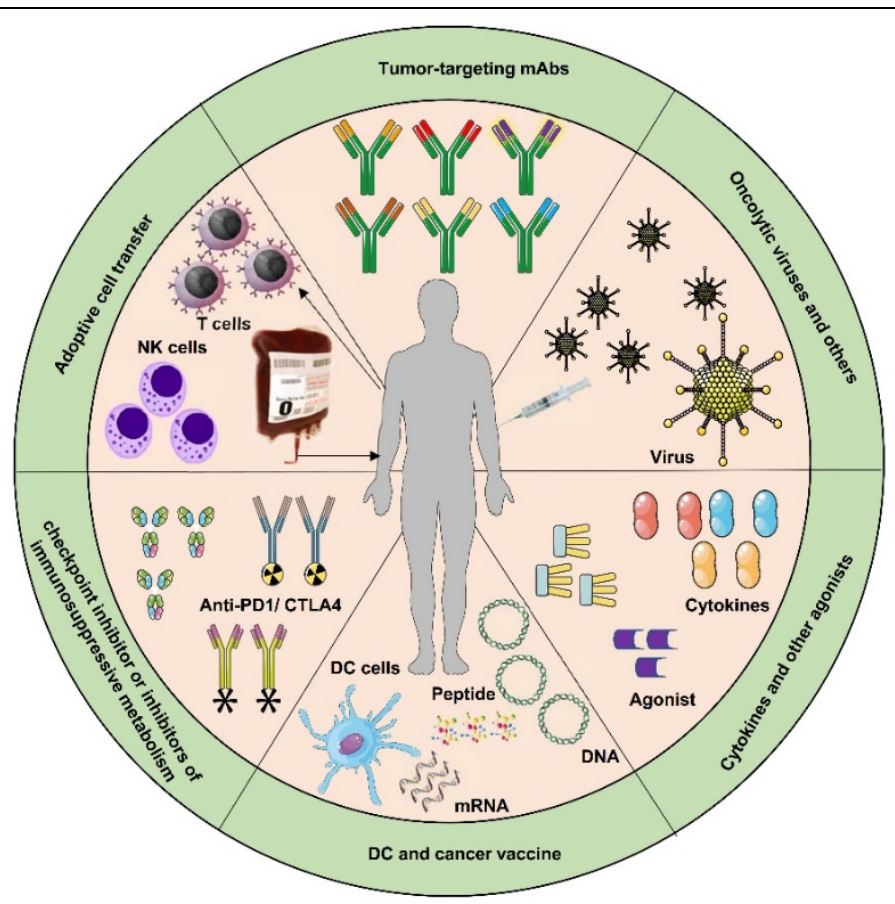

Figure 2. Approaches of anti-cancer Immunotherapy.

Cancer immunotherapy are treatment methods aiming to boost the human immune response against tumor cells. These approaches consist of from nucleotides, proteins, antibodies and cytokines to viral particles and cell products. 


\section{Adoptive cell therapy (ACT): engineered cells combining Chimeric Antigen Receptor (CAR) and Clustered Regularly Interspaced Short Palindromic Repeats (CRISPR) Cas9 technologies}

ACT is a "living cell" drug remedy that involves the procedure of obtaining anti-tumor effector cells (mostly T cell and NK cells [that will be introduced later in more detail]) from the patient itself (autologous) or from a donor (allogeneic). These cells can be expanded or engineered in vitro followed by infusion of immune effector cells back into patients suffering from malignant diseases (Figure 3) [14]. ACT was first reported in 1988, which used tumor-infiltrating lymphocytes (TIL) to melanoma patients and achieved complete regression (CR) [15]. However, obtaining a large number of TIL cells is very difficult for advanced solid tumor patients and not available for hematologic malignancies.

Cytotoxic T cells are found to be potent tumor killer cells that act by recognizing specific tumor antigens by TCR. This approach has been used in engineering for instance a Wilms' Tumor 1 (WT1)-specific TCR on T cells. This approach has demonstrated to be safe and persistent in patients with acute myeloid leukaemia (AML) and myelodysplastic syndromes (MDS) in a clinical trial [16]. Unfortunately, the genetically transferred TCR has been reported to exhibit a mismatch with the endogenous TCR, thus may induce a hazardous T cell mediated self - interaction, known as graft-versus-host disease (GVHD) $[17,18]$. Moreover, the TCR-T cell needs MHC molecules to present antigen, and the MHC molecules may exhibit reduced expression on tumor cells [18]. These disadvantages may restrain the outcome of TCR-T therapy. Therefore, currently, a safe and persistent T-cell-based therapy is urgently required.

Therefore, the introduction of a chimeric antigen receptor (CAR) in T cells (CAR-T) that allows $T$ cells to directly recognize the antigens independent of $\mathrm{MHC}$ molecules and simultaneously activate the $T$ cells, is a powerful alternative concept that gained tremendous attention over the last few years. The structure of a CAR is shown in Figure 4. A CAR contains three compartments: the extracellular antigen-binding part, the transmembrane domain, and the intracellular activation signal. In 2010, the first CAR-T clinical trial was reported, which targeted CD19+ B cell malignancy and achieved complete remission (CR) [19]. In 2017, two CAR-T products, axicabtagene ciloleucel (Yescarta, KITE) and tisagenlecleucel (Kymriah, Novartis) targeting CD19+ B cell lymphoma were approved by the FDA $[20,21]$. However, the treatment comes with severe side effects including the cytokine release syndrome (CRS) [22]. CRS has characteristic clinical presentations, including high fever, hypotension, fatigue, and respiratory distress [22]. As also Immune effector Cell-Associated Neurotoxicity Syndrome (ICANS) [23] and cytopenia [23] occur, novel therapies with fewer side effects are urgently required for $\mathrm{ACT}$ in tumor immunology.

A CAR cell can also be obtained starting from NK cells where the risk of GVHD is absent. In 2020, a phase I/II clinical trial demonstrated that a single infusion of cord blood-derived anti-CD19 CAR-NK cells in CD19+ $\mathrm{B}$ cell lymphoma patients is safe and feasible [24]. After 1 year follow-up, 7 out of 11 patients no CD19+ malignant cells could be detected in the body, which is called complete remission.

In vitro activation of the effector cells allows these cells to be released from the suppressive microenvironment existing in the tumor, boosts their cytotoxicity, and expands them to $10^{10}$ cell numbers [14]. During the expansion period, effector cells can be further tailored or customized towards specific tumors by genetic modification 
methods, including CRISPR/Cas9, viral transduction, and mRNA electroporation $[11,14,25,26]$. Compared to $T$ cells, the genetic modification and expansion of NK cells are more challenging $[27,28]$.

Recently, several study groups have found NK cells can undergo a large-scale expansion in vitro, including a CAR genetic modification (Figure 4) [29-31]. Thus, these unique features of NK cells make them an ideal candidate for ACT.

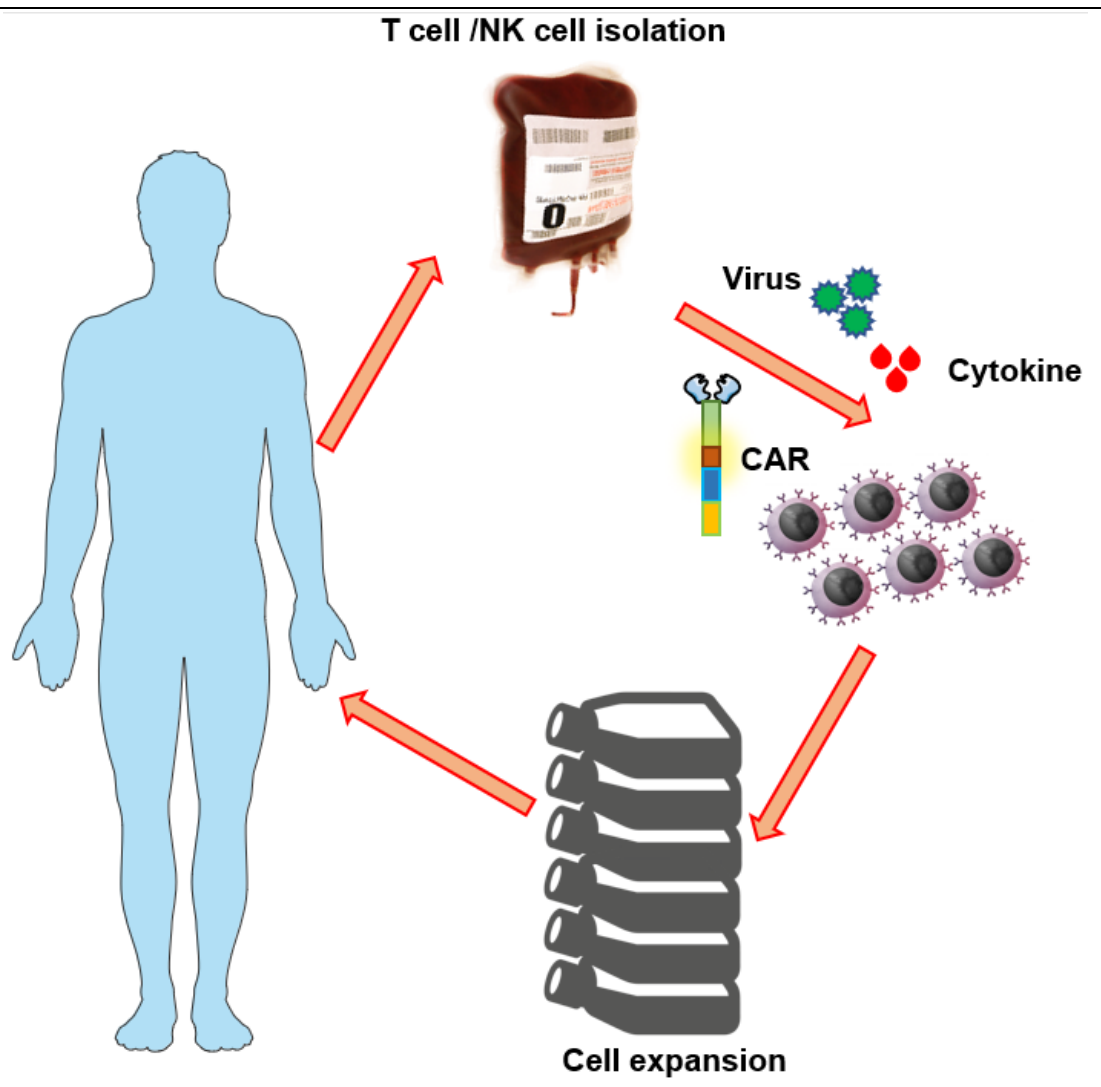

Figure 3. The procedure of adoptive cell therapy.

Adoptive cell therapy obtains adequate high potent effector cells from per example peripheral blood cells, including T cells, NK cells, and monocytes. After isolation, cells are cultured in a cytokine-enriched medium to get stimulated. In the CAR-T generation, genetic modification is also involved in the in vitro expansion procedure. After a few weeks, the effector cells are infused back into cancer patients. 


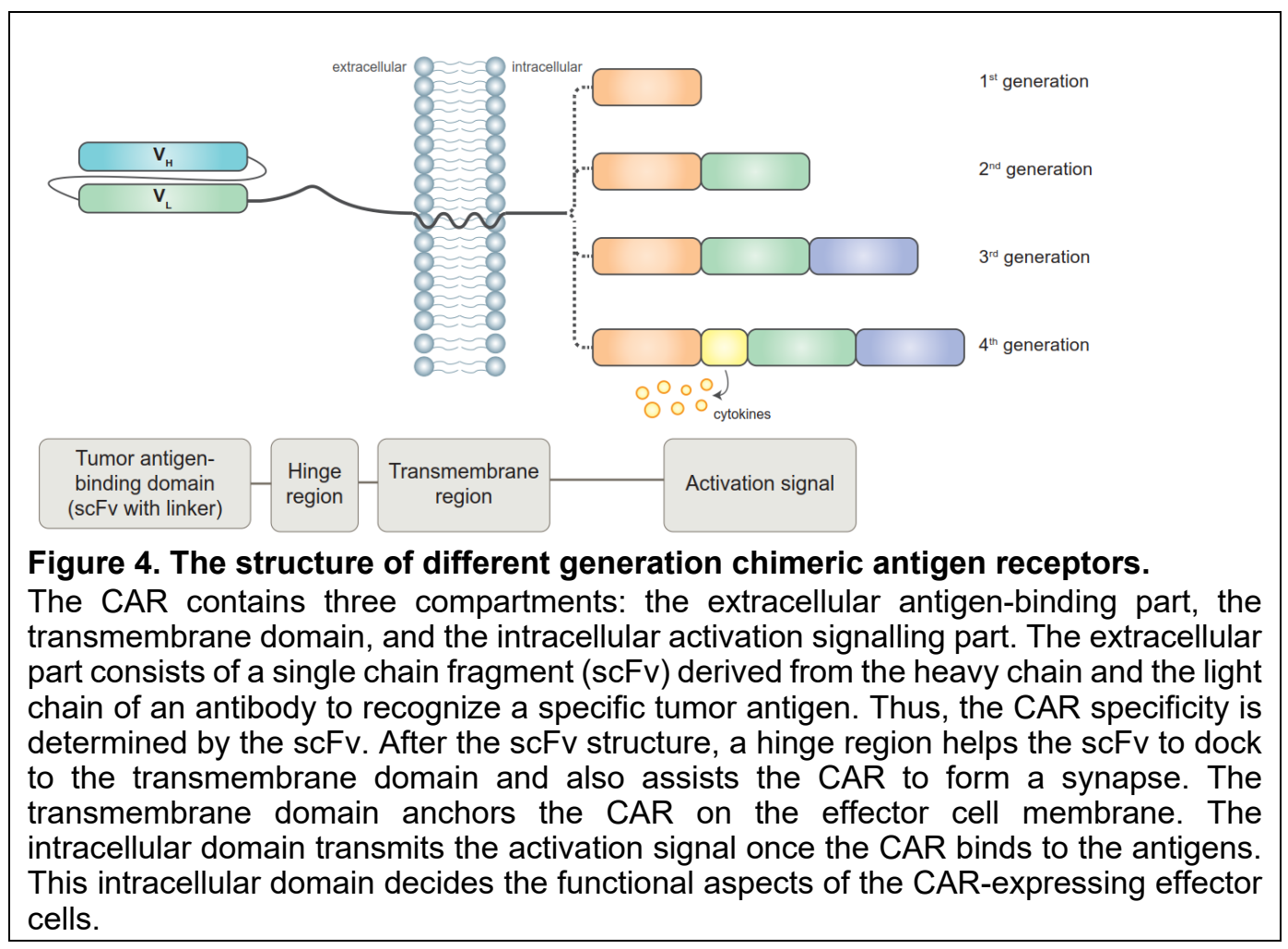

\section{Immunomodulatory mAbs}

As immune cells are dis-regulated in a tumor suppressive microenvironment, immunomodulatory mAbs have been applied to block the inhibitory signals or activate the co-stimulatory pathway, thereby aiming to enhance the persistence of immune cells. Immunomodulatory $\mathrm{mAb}$ is a type of antibody interacting with soluble or cellular components of the immune system.

By now, this therapy can be divided into four general strategies as follows:

(a) Blockade of immunosuppressive receptors expressed on NK cells or T cells (also known as Checkpoint blockade mAbs), such as the killer cell immunoglobulinlike receptor (KIR) family, NKG2A [32,33], CTLA4, PD-1, TIM3, and TIGIT [11,34,35].

(b) The expression of ligands of inhibitory receptors on tumor cells or stromal cells, like the PD-1 ligand CD274 (best known as PD-L1 or B7-H1) [36,37].

(c) The neutralization of $\mathrm{mAb}$ for the immunosuppressive cytokines released in the tumor microenvironment, for example, transforming growth factor (TGF) $\beta 1$, interleukin (IL-10) [11,38].

(d) Agonists of co-stimulatory receptors presented on immune effector cells like tumor necrosis factor receptor superfamily, member 4 (TNFRSF4) (OX40), TNFRSF9 (CD137 or 4-1BB), and TNFRSF18 (GITR) [11]. 
Thus, numerous novel approaches can be achieved by applying immunomodulatory mAbs by boosting the immune cells' anti-cancer ability.

\section{Oncolytic viruses}

Oncolytic viruses are non-pathogenic viral strains that can infect tumor cells and induce apoptosis and demise [39-41]. The anti-tumor potential of oncolytic viruses can be primitive and innately derived from the cytopathic effect, i.e., the deadly overwhelmed cellular metabolism originating from a productive viral infection [42]. These viruses can trigger oncolytic reactions (endogenous or exogenous) due to intracellular gene products (RNA, peptide or protein) that are potentially lethal to the host cells [43]. Since oncolytic viruses possess intrinsic anti-tumor ability, they are considered as passive immunotherapeutic [11]. Packiriswamy et al. demonstrated that oncolytic viral therapy could function as an antigen agnostic vaccine, increasing $\mathrm{CD}^{+}$cytotoxic T-lymphocyte responses against TAAs in patients with multiple myeloma, providing a basis for continued exploration of this modality in combination with immune checkpoint blockade [44].

\section{Cytokines}

Cytokines are polypeptides or glycoproteins that sustain maintenance, promote proliferation, induce differentiation and regulate (via endocrine circuits, paracrine or autocrine) all kinds of biological functions towards immune cells $[45,46]$.

Numerous clinical trials have been conducted to take advantage of cytokines to induce novel immune responses or revive anti-tumor immune responses of immune cells [47]. The treatment regimen of most of the immunostimulatory cytokines as independent therapeutic agents in neoplastic patients is associated with minor (or if any) clinical response [45] because of the presence of similar receptors on the regulatory $T$ cells (Treg) that diminish the stimulation [48]. Thus, conventional cytokines are generally administered as supplementary with other immunotherapeutic treatments.

\section{Dendritic cell-based immunotherapies}

Dendritic cells (DC) are specialized antigen-presenting cells and that present antigens to T cells via major histocompatibility complex (MHC) class I and II molecules. Antigen presentation via binding to specific $T$ cell receptors (TCR) present on $T$ cells and secretion of cytokines will activate antigen-specific $T$ cells. These activated $T$ cells then exert effector functions (i.e. secretion of cytokines to orchestrate the immune response or cytotoxicity) or develop into memory T cells [49].

Several types of DC-based immunotherapies have been developed, which involve the isolation of a patient's circulating monocytes followed by in vitro differentiation/expansion $[50,51]$. DCs are re-infused into patients suffering from a tumor after being primed with tumor-associated antigens (TAAs), in the form of (1) TAA peptides, (2) messenger RNAs (mRNAs) coding TAA, (3) TAA expressing plasmid vectors, (4) bulk cancer cell lysates or (5) bulk cancer cell-derived mRNA [52,53]. 
It has been reported that DC cells cross-talk with NK cells, which can be mutually beneficial and increases their anti-tumor activities [54-56]. Therefore, DC cell-based immunotherapy can be considered a powerful anti-cancer immunotherapy.

\section{Tumor antigen-specific antibody}

Monoclonal antibodies (mAb) have been widely applied in clinical cancer therapy for decades and have shown promising progress [57]. MAbs contain a Fab part and an Fc fragment [58]. The Fab part contains the antigen-binding domains, which can recognize specific epitopes, whereas the Fc tail conjugates with the Fc receptors, which are expressed on human NK cells, macrophages and monocytes $[59,60]$. When the effector cells bind to an antibody, which is specific towards the antigen expressed on target cells, the effector cells get activated and lyse the target cells. This process is called antibodydependent cellular cytotoxicity (ADCC) [61].

Both the American Food and Drug Administration (FDA) and European Medicine Agency (EMA) approved clinical mAbs are typically used as a combination of remedies in guiding cytotoxic effector cells, specifically towards tumor antigens expressed on neoplastic cells [57]. Tumor-specific antibodies interact with the components of the immune system through complement-dependent cytotoxicity (CDC) [62], ADCC [63], antibodydependent cellular phagocytosis (ADCP) and dampen signal transduction in the cancerous cell or eradicate antigens in vivo $[57,64,65]$. mAb can also be engineered with payloads (e.g., radioisotopes, drugs, or toxins) to specifically and efficiently kill tumor cells or to stimulate prodrugs precisely within the tumor foci (antibody-directed enzyme prodrug therapy, ADEPT) $[57,62]$.

$\mathrm{mAbs}$ can be conjugated with conventional chemotherapeutic drugs, which endows the specificity of the therapy without damaging the healthy cells [66]. Recently, engineered $\mathrm{mAb}$ with bi-specific antibodies or recombinant with cytokines, known as tri-specific killer engager (TriKE), have been found to exert strong anti-cancer immune responses $[27,67]$. The TriKE consists of two single-chain variable fragments (scFvs) at the two ends, one directing CD16 (also called Fc-gamma receptor) on NK cells and the other targeting antigen on tumor cells, linked by an interleukin-15 (IL-15) in the middle to enhance activation and proliferation of NK cell [68].

EMA and FDA-approved mAbs have exerted exciting progress in cancer remedies that render the scientists increased confidence to invent more mAbs targeting neoantigens $[57,65]$. However, there are a few limitations of antibody therapy, including the general need for continued administration - although in many cases only a temporarily administration is given - and its restrained efficacy when there is a high mutation in tumor antigens.

\section{NK cell biology}

NK cells are a group of bone marrow-derived innate lymphocytes present in most organs of the human body. A large proportion of NK cells exist in the peripheral blood where they make up about $10 \%$ of all immune cells [69]. NK cells are characterized phenotypically as $\mathrm{CD}^{-}{ }^{-} \mathrm{CD} 56^{+}$large granular lymphocytes in humans, which contain powerful lytic granules that were initially defined by their activity to kill tumor cells or 
abnormal cells without prior sensitization [70]. The functional role of NK cells has since been broadened to include the elimination of virally infected cells, senescent cells, and production of cytokines that intercede regulation and cross-talk of adjacent immune cells [71]. Throughout several decades, scientists have been fascinated by the mechanism by which NK cells can detect and kill tumorigenic or virally-infected cells while tolerating healthy self-tissue [27]. In the early 1990s, Kärre initialized the "missing self" theory that hypothesized that NK cells are capable of identifying reduced or missing in surface expression of MHC class I proteins [human leukocyte antigens (HLA) class I in human] inducing recognition as 'non-self' [72]. This hypothesis was based on studies involved in the discoveries of suppressive receptors (i.e., NKG2A, KIR) that can bind with the $\mathrm{MHC}$ I molecules and restrain the killing capacity of NK cells; thus, protecting the healthy cells [73]. At the same time, cancer cells often down regulate HLA molecules to avoid detection and recognition by T cells, but the NK cells are sensitive to the reduced or lacked HLA on tumor cells. Thus, NK cells are regarded as important anti-cancer immune cells [74]. Like T cell maturation, matured NK cells also pass through a selftolerance process called NK cell education to get the ability to distinguish non-selfantigens from self-antigens. The function of mature NK cells is determined by the signals from activating or inhibitory receptors (Figure 5).

\section{Education and Licensing of NK cells}

NK cell education is a quantitative procedure in which NK cells obtain their effective capacity through frequent and consistent interaction with self-MHC class I. This interaction occurs through its inhibitory receptors (KIR family, NKG2A/CD94, and others.) [75].

Education of NK cells include licensing, disarming, rheostat and tuning. After the education process, NK cells become potent killer cells towards foreign pathogens [76]. Licensing, which is one of the most important education processes, requires a positive role for MHC-dependent inhibitory receptors on NK cells and involves the intracellular inhibitory motif (ITIM) in effector responses [77,78]. It results in two kinds of self-tolerant NK cells, which include licensed (armed) and unlicensed (disarmed) cells, and may provide new concepts for investigating NK cells in immunotherapy [79]. Mature NK cells express KIR receptors (licensed NK cells) that will restrain its function when engaged with MHC molecules to avoid attacking self-tissue [80]. These licensed NK cells exert a stronger killing capacity against target cells when compared to unlicensed NK cells. The licensing influence is less remarkable in pre-activated NK cells, indicating that licensing requirements could be alleviated under some particular surroundings [81]. Unlicensed NK cells may be involved in response to pro-inflammatory cytokines during virus infections [78]. The whole process of NK cell education is not entirely understood; further investigation is still required $[76,82]$.

\section{Activation receptors}

NK cells contain various receptors that can promote their effector activities when they act alone or in cross-talk with other immune cells [83]. Cytokine receptors are abundantly expressed on NK cells, which deliver the activation signal to trigger proliferation and functional activities of NK cells [84]. NKG2D (also known as CD314 and KLRK1), 
cytokine receptors, natural cytotoxicity receptors (NCRs), and CD16 are the activating NK cell receptors engaged in immune responses against tumor [85]. The type II transmembrane protein, NKG2D, has unique characteristics, including binding to a broad range of ligands on the target cells [86]. In humans, NKG2D ligands (NKG2DLs) include $\mathrm{MHC}$ class I polypeptide-related sequence (MIC): MICA and MICB, retinoic acid early transcript 1 protein (RAET1) family protein: RAET1E, RAET1G, RAET1H, RAET1I, RAET1L, and RAET1N (alternative name ULBP1-ULBP6) [86]. These NKG2DLs are not expressed on the normal cell surface but can be presented on senescent cells, virusinfected cells, deformed cells, and cancer cells [86].

NCRs are another family of receptors that give activation signals and contain three receptors: NKp30 (also known as CD337 or NCR3), NKp44 (CD336 or NCR2), and NKp46 (CD335 or NCR1). NKp46 and NKp30 are expressed constitutively on human NK cells, while NKp44 is only presented after the activation of NK cells [67,74].

An extensive array of NCR ligands has been discovered, including ligands encoded by genes from microorganisms like viruses, bacteria, and parasites, but most of them are not well known [71]. NCR ligands have been proven to be expressed in various cancer cells and minor healthy tissues [71]. A potent activator of NK cells is CD16 (also known as Fc region gamma receptor IIIA, FcyRIIla), which is expressed predominantly on the CD56dim subpopulation of NK cells in humans [87]. This receptor can bind with the constant region $(\mathrm{Fc})$ of $\lg \mathrm{G}$ antibodies, mostly with $\lg \mathrm{G} 1$ in humans, and engage in ADCC [70]. These activating receptors can help the NK cells to detect tumor cells and cause cytotoxicity, thus eliminating cancer cells (Figure 5). 


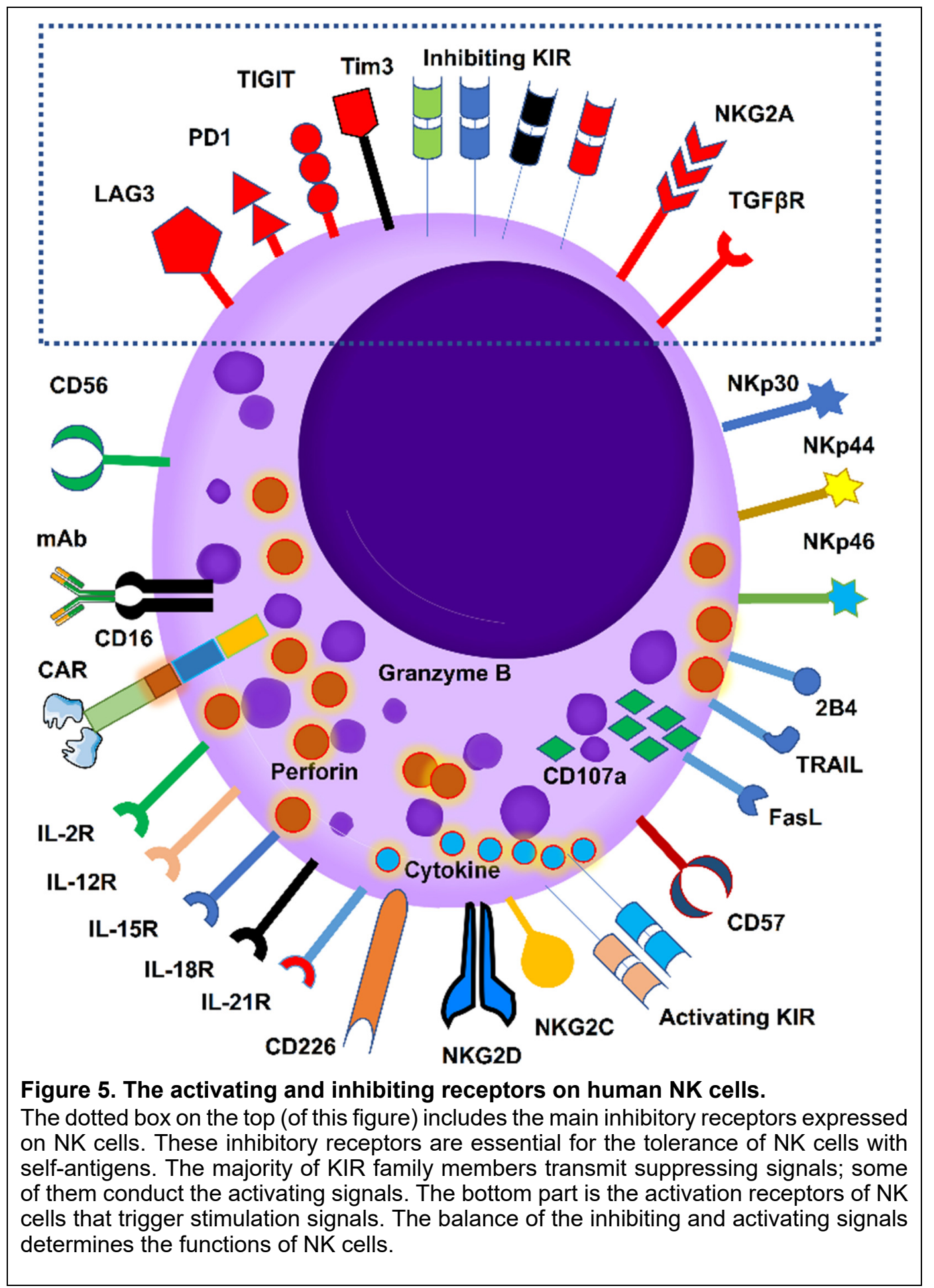




\section{Inhibitory receptors}

Inhibitory receptors expressed on NK cells convey a suppressive signal to reduce the effective immune response and prevent the killing of healthy cells (self-cells) [73]. NK cells express suppressive receptors that bind to MHC class I molecules, including the KIR. The KIR receptors are encoded by a multigenic family of polymorphic genes [70]. Some KIR family members also deliver the activation signal [69].

Besides KIR, another inhibitory receptor known as NKG2A binds with CD94 and forms a heterodimer. Unlike KIRs, the non-polymorphic MHC class I ligand, HLA-E is the ligand for NKG2A CD94 heterodimer [87]. TGF $\beta$ is an important member of the suppressive tumor microenvironment and can bind with the TGF $\beta$ receptor on the NK cell to transmit the inhibitory signal [88]. The inhibitory receptors assist the NK cells in tolerating self$\mathrm{MHC}$ class I ligands on healthy cells and restrain their activation [87]. Moreover, the inhibitory receptors are important "switches" that restrain the function of NK cells. The inhibitory receptors are depicted in Figure 5.

\section{NK cell Memory}

The ability of the human immune system to act swiftly, precisely, and provide enhanced protection towards previously engaged antigens is defined as immunological memory [89].

T cells and B cells are adaptive immune cells and can induce immune memory, due to which they have a long lifespan and strong proliferating ability. In contrast, NK cells are innate immune cells that can immediately kill external pathogens without prior stimulation. Previously, NK cells were considered to have no memory [89].

However, recently, NK cells are considered to have many developmental and functional characteristics similar to cells from the adaptive immune system [71].

NK cells were first shown to acquire antigen-specific acute and "memory" immune responses in a mice model of hapten (2,4-dinitrofluorobenzene or oxazolone)-mediated contact hypersensitivity. Treatment of Rag2-/- mice which lacked functional B or T cells, with a specific hapten induced an NK cell-dependent increased swelling upon treatment of the mouse's ear with the same hapten, which lasted for at least 4 weeks [90,91].

NK cells express numerous germline-encoded activating receptors, which render NK cells to conjugate with ligands of stress-induced or pathogen-derived self-antigens [92]. Emerging data showed that innate immune cells like NK cells could also possess memory, further enhancing the anti-tumor ability [93-95]. With the development of different technologies, scientists have investigated that NK cells can also acquire functional memory in response to cytokine stimulation [95,96], abnormal pathogens (especially in viral antigens), and homeostatic proliferation $[71,89]$. Gang et al. reported that CAR-modified memory-like NK cells exhibit potent responses to NK-resistant lymphomas; these memory-like NK cells were stimulated with IL-12, IL-15, and IL-18. The memory-like NK cells functioned better than NK cells treated with IL-15 alone [97]. Further, NK cells can also be trained to acquire a memory that could be applied in cancer immunotherapy [93]. 


\section{Tumor Microenvironment limits the function of NK cells}

In all tumors, the inhibitory tumor microenvironment may account for restraining the antitumor activity of NK cells $[98,99]$.

The tumor microenvironment is comprised of cancer cells, fibroblasts, stromal cells, endothelial cells, regulatory immune cells (e.g., Treg) and small molecules (lactate, hypoxia, low $\mathrm{PH}, \mathrm{CD} 47, \mathrm{HLA}-\mathrm{E}$, etc.) that promote tumor progression but is detrimental for the NK cells [100]. Hypoxia is a common feature of most tumors, and tumor cells adapt to this condition by upregulating the transcription factor Hypoxia-Inducible Factor (HIF)-1a. Conditional knockout of HIF-1a in NK cells of mice resulted in reduced tumor growth, up-regulated expression or activation of phenotypic markers, effector molecules, and an enriched NF-кB pathway in tumor-infiltrating NK cells [101]. Degos et al. showed that in the endometrial tumor microenvironment, concentrations of both chemokines (CXCL12, IP-1, and CCL27) and proinflammatory cytokines (IL-1 $\beta$ and IL-6) were increased that might inhibit NK cell function and recruitment to the tumor site [102]. Harmon et al. reported that lactate-mediated acidification of tumor microenvironment promotes apoptosis of liver-resident NK cells in a colorectal liver metastasis model [103]. Nath et al. found that NK cell recruitment and activation are regulated by CD47 expression in the tumor microenvironment [104]. Thus, the tumor microenvironment is an obstacle that constrains the anti-tumor activity of NK cells.

\section{Outline of the Thesis}

Our group has been investigating the generation of more powerful NK cells for cancer immunotherapy for two decades. In this thesis, we follow our previous experience and capitalize on recent technological developments to enhance anti-tumor responses in human NK cells.

As tumor models, we mainly rely on MUC1-positive leukemia and breast cancer tumor cell lines. MUC1 is a membrane glycoprotein that abundantly express on epithelial cells with long tandem repeats and side-chains [105]. In healthy tissues, MUC1 is heavily Oglycosylated, with $50-90 \%$ of the protein weight being carbohydrate. During neoplastic transformation, MUC1 is overexpressed, and as a result of altered glycosyltransferase activity, it undergoes incomplete glycosylation [106]. Cancer cells with Cosmc gene mutation induce the tumor-specific MUC1 epitopes with carbohydrate antigens, Tn, and sialyl Tn (STn) [107]. Several antibodies for cancerous MUC1 have been applied in clinical trials; however, the results of the trials were non-significant [108-110]. Thus, the identification of a novel antibody for MUC1 will be beneficial for controlling MUC1 positive tumors.

In this thesis, we aim to generate novel and effective approaches to enhance the killing capacity of the NK cells for tumor immunotherapy. Besides antibody combination, we also try to use CAR-equipped NK cells and CRISPR/Cas9 technologies to augment the functional ability of NK cells further.

In Chapter 2, we will continue the previous work of our group to investigate the humanized anti-MUC1-Tn/STn antibodies. The MUC1-Tn epitope is expressed only on tumor cells which have been considered as a promising tumor target. We generated a 
humanized anti-MUC1-Tn/STn based on the murine 5E5 antibody. We will investigate whether our humanized antibody can recognize MUC1-Tn/STn epitopes on MUC-Tn+ cancer cell lines and bind to NK cells. Furthermore, we will further study whether the humanized antibody can induce ADCC and trigger degranulation. From a previous study, we know that MUC1 expression on tumor cells is dynamically regulated through the endocytosis process [111]. Thus, we will test whether increasing the MUC-Tn epitopes on the tumor cell surface by endocytosis inhibitors can boost the ADCC. These results of anti-MUC1-mAb may pave the way to generating a CAR-NK cell, which may improve the killing capacity of NK cells against MUC1 positive tumors.

In Chapter 3, we aim to find novel approaches to enhance the viral transduction efficiency in the NK cells. Low density lipoprotein receptor (LDLR) has been demonstrated to be the receptor of vesicular stomatitis virus (VSV) [112] and is expressed only at low levels on NK cells. We will test the potential of statins and nonstatin compounds to increase the LDLR expression on human primary NK cells. Simultaneously, we will explore whether the statins or compounds will have an impact on the phenotype and function of the NK cells.

In Chapter 4, we will summarize the current published CAR-NK studies, especially the design and generation of CAR-NK. We are aiming to make the CAR-NK technology easily interpretable and achievable. We will discuss all the details of designing a functional CAR, the different source of NK cells, methods to expand NK cells, approaches to enhancing the transduction on NK cells. This should help in creating our own CAR-NK cells. We will give an overview of the experiments that we have conducted on generating anti-MUC1 CAR-NK cells.

As discussed before, the function of NK cells is inhibited in the tumor microenvironment. NK cells express NKG2A, one of the dominant inhibitory receptor, which binds to HLAE molecule on target cells [113]. Antibody blockade on NKG2A has also been reported to improve the treatment outcome [32].

In Chapter 5, we are going to compare the blockade efficiency of antibody and CRISPR/cas9 mediated gene ablation to diminish the NKG2A inhibitory signal in primarily human NK cells. Meanwhile, we will also investigate the phenotype, functional markers and transcriptome of NK cells that lack NKG2A.

In Chapter 6, we will summarize all the observations and the findings of this thesis in a general discussion and provide an outlook and perspective of NK cell-based tumor immunotherapy in the future. 


\section{References}

1. Sung H, Ferlay J, Siegel RL, Laversanne M, Soerjomataram I, Jemal A, et al. Global cancer statistics 2020: Globocan estimates of incidence and mortality worldwide for 36 cancers in 185 countries. CA Cancer J Clin. 2021. doi:10.3322/caac.21660.

2. Reya T, Morrison SJ, Clarke MF, Weissman IL. Stem cells, cancer, and cancer stem cells. Nature. 2001; 414:105-111. doi:10.1038/35102167.

3. Jiang WG, Sanders AJ, Katoh M, Ungefroren H, Gieseler F, Prince M, et al. Tissue invasion and metastasis: Molecular, biological and clinical perspectives. Seminars in Cancer Biology. 2015; 35:S244-S275. doi:https://doi.org/10.1016/j.semcancer.2015.03.008.

4. Lambert AW, Pattabiraman DR, Weinberg RA. Emerging biological principles of metastasis. Cell. 2017; 168:670-691. doi:10.1016/j.cell.2016.11.037.

5. June $\mathrm{CH}$, Sadelain M. Chimeric antigen receptor therapy. N Engl J Med. 2018; 379:64-73. doi:10.1056/NEJMra1706169.

6. Parkin J, Cohen B. An overview of the immune system. Lancet. 2001; 357:1777-1789. doi:10.1016/s01406736(00)04904-7.

7. Gourbal B, Pinaud S, Beckers GJM, Van Der Meer JWM, Conrath U, Netea MG. Innate immune memory: An evolutionary perspective. Immunol Rev. 2018; 283:21-40. doi:10.1111/imr.12647.

8. Bonilla FA, Oettgen HC. Adaptive immunity. J Allergy Clin Immunol. 2010; 125:S33-40. doi:10.1016/j.jaci.2009.09.017.

9. Demaria O, Cornen S, Daëron M, Morel Y, Medzhitov R, Vivier E. Harnessing innate immunity in cancer therapy. Nature. 2019; 574:45-56. doi:10.1038/s41586-019-1593-5.

10. Netea MG, Schlitzer A, Placek K, Joosten LAB, Schultze JL. Innate and adaptive immune memory: An evolutionary continuum in the host's response to pathogens. Cell Host Microbe. 2019; 25:13-26. doi:10.1016/j.chom.2018.12.006.

11. Galluzzi L, Vacchelli E, Bravo-San Pedro JM, Buqué A, Senovilla L, Baracco EE, et al. Classification of current anticancer immunotherapies. Oncotarget. 2014; 5:12472-12508. doi:10.18632/oncotarget.2998.

12. Dunbar CE, High KA, Joung JK, Kohn DB, Ozawa K, Sadelain M. Gene therapy comes of age. Science. 2018; 359:eaan4672. doi:10.1126/science.aan4672.

13. Couzin-Frankel J. Breakthrough of the year 2013. Cancer immunotherapy. Science. 2013; 342:1432-1433. doi:10.1126/science.342.6165.1432.

14. Rosenberg SA, Restifo NP. Adoptive cell transfer as personalized immunotherapy for human cancer. Science. 2015; 348:62-68. doi:10.1126/science.aaa4967.

15. Rosenberg SA, Packard BS, Aebersold PM, Solomon D, Topalian SL, Toy ST, et al. Use of tumor-infiltrating lymphocytes and interleukin-2 in the immunotherapy of patients with metastatic melanoma. A preliminary report. N Engl J Med. 1988; 319:1676-1680. doi:10.1056/nejm198812223192527.

16. Tawara I, Kageyama S, Miyahara Y, Fujiwara H, Nishida T, Akatsuka Y, et al. Safety and persistence of wt1-specific t-cell receptor gene-transduced lymphocytes in patients with aml and mds. Blood. 2017; 130:1985-1994. doi:10.1182/blood-2017-06-791202.

17. Goebeler ME, Bargou RC. T cell-engaging therapies - bites and beyond. Nat Rev Clin Oncol. 2020; 17:418434. doi:10.1038/s41571-020-0347-5.

18. Jiang $\mathrm{X}, \mathrm{Xu} \mathrm{J}$, Liu $\mathrm{M}$, Xing $\mathrm{H}$, Wang Z, Huang L, et al. Adoptive cd8(+) $\mathrm{t}$ cell therapy against cancer:Challenges and opportunities. Cancer Lett. 2019; 462:23-32. doi:10.1016/j.canlet.2019.07.017.

19. Kochenderfer JN, Wilson WH, Janik JE, Dudley ME, Stetler-Stevenson M, Feldman SA, et al. Eradication of $b$-lineage cells and regression of lymphoma in a patient treated with autologous $t$ cells genetically engineered to recognize cd19. Blood. 2010; 116:4099-4102. doi:10.1182/blood-2010-04-281931.

20. Geyer MB. First car to pass the road test: Tisagenlecleucel's drive to fda approval. Clin Cancer Res. 2019; 25:1133-1135. doi:10.1158/1078-0432.ccr-18-3328.

21. Bouchkouj N, Kasamon YL, de Claro RA, George B, Lin X, Lee S, et al. Fda approval summary: Axicabtagene ciloleucel for relapsed or refractory large b-cell lymphoma. Clin Cancer Res. 2019; 25:17021708. doi:10.1158/1078-0432.ccr-18-2743.

22. Liu D, Zhao J. Cytokine release syndrome: Grading, modeling, and new therapy. J Hematol Oncol. 2018; 11:121. doi:10.1186/s13045-018-0653-x.

23. Schubert ML, Schmitt M, Wang L, Ramos CA, Jordan K, Müller-Tidow C, et al. Side-effect management of chimeric antigen receptor (car) t-cell therapy. Ann Oncol. 2021; 32:34-48. doi:10.1016/j.annonc.2020.10.478.

24. Liu E, Marin D, Banerjee P, Macapinlac HA, Thompson P, Basar R, et al. Use of car-transduced natural killer cells in cd19-positive lymphoid tumors. N Engl J Med. 2020; 382:545-553. doi:10.1056/NEJMoa1910607.

25. Guedan S, Ruella M, June CH. Emerging cellular therapies for cancer. Annu Rev Immunol. 2019; 37:145171. doi:10.1146/annurev-immunol-042718-041407. 
26. Xu X, Li T, Shen S, Wang J, Abdou P, Gu Z, et al. Advances in engineering cells for cancer immunotherapy. Theranostics. 2019; 9:7889-7905. doi:10.7150/thno.38583.

27. Miller JS, Lanier LL. Natural killer cells in cancer immunotherapy. Annu Rev Cancer Biol. 2019; 3:77-103. doi:10.1146/annurev-cancerbio-030518-055653.

28. Bari R, Granzin M, Tsang KS, Roy A, Krueger W, Orentas R, et al. A distinct subset of highly proliferative and lentiviral vector (Iv)-transducible nk cells define a readily engineered subset for adoptive cellular therapy. Front Immunol. 2019; 10:2001. doi:10.3389/fimmu.2019.02001.

29. Denman CJ, Senyukov VV, Somanchi SS, Phatarpekar PV, Kopp LM, Johnson JL, et al. Membrane-bound il-21 promotes sustained ex vivo proliferation of human natural killer cells. PLoS One. 2012; 7:e30264. doi:10.1371/journal.pone.0030264.

30. Huang RS, Lai MC, Shih HA, Lin S. A robust platform for expansion and genome editing of primary human natural killer cells. J Exp Med. 2021; 218. doi:10.1084/jem.20201529.

31. Pomeroy EJ, Hunzeker JT, Kluesner MG, Lahr WS, Smeester BA, Crosby MR, et al. A genetically engineered primary human natural killer cell platform for cancer immunotherapy. Mol Ther. 2020; 28:5263. doi:https://doi.org/10.1016/j.ymthe.2019.10.009.

32. van Hall T, André P, Horowitz A, Ruan DF, Borst L, Zerbib R, et al. Monalizumab: Inhibiting the novel immune checkpoint nkg2a. J Immunother Cancer. 2019; 7:263. doi:10.1186/s40425-019-0761-3.

33. André P, Denis C, Soulas C, Bourbon-Caillet C, Lopez J, Arnoux T, et al. Anti-nkg2a mab is a checkpoint inhibitor that promotes anti-tumor immunity by unleashing both $t$ and nk cells. Cell. 2018; 175:17311743.e1713. doi:10.1016/j.cell.2018.10.014.

34. Wang M, Liu Y, Cheng Y, Wei Y, Wei X. Immune checkpoint blockade and its combination therapy with small-molecule inhibitors for cancer treatment. Biochim Biophys Acta Rev Cancer. 2019; 1871:199-224. doi:10.1016/j.bbcan.2018.12.002.

35. Darvin P, Toor SM, Sasidharan Nair V, Elkord E. Immune checkpoint inhibitors: Recent progress and potential biomarkers. Exp Mol Med. 2018; 50:1-11. doi:10.1038/s12276-018-0191-1.

36. Olivo Pimentel V, Marcus D, van der Wiel AM, Lieuwes NG, Biemans R, Lieverse RI, et al. Releasing the brakes of tumor immunity with anti-pd-I1 and pushing its accelerator with I19-il2 cures poorly immunogenic tumors when combined with radiotherapy. J Immunother Cancer. 2021; 9. doi:10.1136/jitc-2020-001764.

37. Rodriguez-Barbosa JI, Azuma M, Zelinskyy G, Perez-Simon JA, Del Rio ML. Critical role of pd-I1 expression on non-tumor cells rather than on tumor cells for effective anti-pd-I1 immunotherapy in a transplantable mouse hematopoietic tumor model. Cancer Immunol Immunother. 2020; 69:1001-1014. doi:10.1007/s00262-020-02520-z.

38. Hui L, Chen Y. Tumor microenvironment: Sanctuary of the devil. Cancer Lett. 2015; 368:7-13. doi:10.1016/j.canlet.2015.07.039.

39. Raja J, Ludwig JM, Gettinger SN, Schalper KA, Kim HS. Oncolytic virus immunotherapy: Future prospects for oncology. J Immunother Cancer. 2018; 6:140. doi:10.1186/s40425-018-0458-z.

40. Lee P, Gujar S. Potentiating prostate cancer immunotherapy with oncolytic viruses. Nat Rev Urol. 2018; 15:235-250. doi:10.1038/nrurol.2018.10.

41. Bommareddy PK, Shettigar M, Kaufman HL. Integrating oncolytic viruses in combination cancer immunotherapy. Nat Rev Immunol. 2018; 18:498-513. doi:10.1038/s41577-018-0014-6.

42. Twumasi-Boateng K, Pettigrew JL, Kwok YYE, Bell JC, Nelson BH. Oncolytic viruses as engineering platforms for combination immunotherapy. Nat Rev Cancer. 2018; 18:419-432. doi:10.1038/s41568-0180009-4.

43. Hemminki O, Dos Santos JM, Hemminki A. Oncolytic viruses for cancer immunotherapy. J Hematol Oncol. 2020; 13:84. doi:10.1186/s13045-020-00922-1.

44. Packiriswamy N, Upreti D, Zhou Y, Khan R, Miller A, Diaz RM, et al. Oncolytic measles virus therapy enhances tumor antigen-specific t-cell responses in patients with multiple myeloma. Leukemia. 2020; 34:3310-3322. doi:10.1038/s41375-020-0828-7.

45. Waldmann TA. Cytokines in cancer immunotherapy. Cold Spring Harb Perspect Biol. $2018 ; 10$. doi:10.1101/cshperspect.a028472.

46. Dranoff G. Cytokines in cancer pathogenesis and cancer therapy. Nat Rev Cancer. 2004; 4:11-22. doi:10.1038/nrc1252.

47. Berraondo P, Sanmamed MF, Ochoa MC, Etxeberria I, Aznar MA, Pérez-Gracia JL, et al. Cytokines in clinical cancer immunotherapy. Br J Cancer. 2019; 120:6-15. doi:10.1038/s41416-018-0328-y.

48. Derynck R, Turley SJ, Akhurst RJ. Tgf $\beta$ biology in cancer progression and immunotherapy. Nat Rev Clin Oncol. 2021; 18:9-34. doi:10.1038/s41571-020-0403-1.

49. Oth T, Vanderlocht J, Van Elssen CH, Bos GM, Germeraad WT. Pathogen-associated molecular patterns induced crosstalk between dendritic cells, thelper cells, and natural killer helper cells can improve dendritic cell vaccination. Mediators Inflamm. 2016; 2016:5740373. doi:10.1155/2016/5740373.

50. Waldman AD, Fritz JM, Lenardo MJ. A guide to cancer immunotherapy: From t cell basic science to clinical practice. Nat Rev Immunol. 2020; 20:651-668. doi:10.1038/s41577-020-0306-5.

51. Kurzrock R, Kantarjian HM, Kesselheim AS, Sigal EV. New drug approvals in oncology. Nat Rev Clin Oncol. 2020; 17:140-146. doi:10.1038/s41571-019-0313-2. 
52. Carreno BM, Magrini V, Becker-Hapak M, Kaabinejadian S, Hundal J, Petti AA, et al. Cancer immunotherapy. A dendritic cell vaccine increases the breadth and diversity of melanoma neoantigenspecific t cells. Science. 2015; 348:803-808. doi:10.1126/science.aaa3828.

53. Palucka K, Banchereau J. Cancer immunotherapy via dendritic cells. Nat Rev Cancer. 2012; $12: 265-277$. doi:10.1038/nrc3258.

54. Van Elssen CH, Oth T, Germeraad WT, Bos GM, Vanderlocht J. Natural killer cells: The secret weapon in dendritic cell vaccination strategies. Clin Cancer Res. 2014; 20:1095-1103. doi:10.1158/1078-0432.Ccr13-2302.

55. Van Elssen CH, Vanderlocht J, Oth T, Senden-Gijsbers BL, Germeraad WT, Bos GM. Inflammationrestraining effects of prostaglandin e2 on natural killer-dendritic cell (nk-dc) interaction are imprinted during dc maturation. Blood. 2011; 118:2473-2482. doi:10.1182/blood-2010-09-307835.

56. Van Elssen $\mathrm{CH}$, Vanderlocht J, Frings PW, Senden-Gijsbers BL, Schnijderberg MC, van Gelder M, et al. Klebsiella pneumoniae-triggered dc recruit human $\mathrm{nk}$ cells in a ccr5-dependent manner leading to increased ccl19-responsiveness and activation of nk cells. Eur J Immunol. 2010; 40:3138-3149. doi:10.1002/eji.201040496.

57. Adams GP, Weiner LM. Monoclonal antibody therapy of cancer. Nat Biotechnol. 2005; 23:1147-1157. doi:10.1038/nbt1137.

58. Singh S, Kumar NK, Dwiwedi P, Charan J, Kaur R, Sidhu P, et al. Monoclonal antibodies: A review. Curr Clin Pharmacol. 2018; 13:85-99. doi:10.2174/1574884712666170809124728.

59. Gauthier M, Laroye C, Bensoussan D, Boura C, Decot V. Natural killer cells and monoclonal antibodies: Two partners for successful antibody dependent cytotoxicity against tumor cells. Critical Reviews in Oncology/Hematology. 2021; 160:103261. doi:https://doi.org/10.1016/j.critrevonc.2021.103261.

60. Anderson NR, Minutolo NG, Gill S, Klichinsky M. Macrophage-based approaches for cancer immunotherapy. Cancer Res. 2021; 81:1201-1208. doi:10.1158/0008-5472.can-20-2990.

61. Goydel RS, Rader C. Antibody-based cancer therapy. Oncogene. 2021; 40:3655-3664. doi:10.1038/s41388-021-01811-8.

62. Nicodemus CF. Antibody-based immunotherapy of solid cancers: Progress and possibilities. Immunotherapy. 2015; 7:923-939. doi:10.2217/imt.15.57.

63. Ochoa MC, Minute L, Rodriguez I, Garasa S, Perez-Ruiz E, Inogés S, et al. Antibody-dependent cell cytotoxicity: Immunotherapy strategies enhancing effector nk cells. Immunol Cell Biol. 2017; 95:347-355. doi:10.1038/icb.2017.6.

64. Rader C. Bispecific antibodies in cancer immunotherapy. Curr Opin Biotechnol. 2020; 65:9-16. doi:10.1016/j.copbio.2019.11.020.

65. Yu S, Li A, Liu Q, Yuan X, Xu H, Jiao D, et al. Recent advances of bispecific antibodies in solid tumors. J Hematol Oncol. 2017; 10:155. doi:10.1186/s13045-017-0522-z.

66. Kaplon H, Muralidharan M, Schneider Z, Reichert JM. Antibodies to watch in 2020. mAbs. 2020; 12:1703531. doi:10.1080/19420862.2019.1703531.

67. Myers JA, Miller JS. Exploring the nk cell platform for cancer immunotherapy. Nat Rev Clin Oncol. 2020. doi:10.1038/s41571-020-0426-7.

68. Sarhan D, Brandt L, Felices M, Guldevall K, Lenvik T, Hinderlie P, et al. 161533 trike stimulates nk-cell function to overcome myeloid-derived suppressor cells in mds. Blood Adv. 2018; 2:1459-1469. doi:10.1182/bloodadvances.2017012369.

69. Guillerey C, Huntington ND, Smyth MJ. Targeting natural killer cells in cancer immunotherapy. Nat Immunol. 2016; 17:1025. doi:10.1038/ni.3518.

70. Morvan MG, Lanier LL. Nk cells and cancer: You can teach innate cells new tricks. Nat Rev Cancer. 2015; 16:7. doi:10.1038/nrc.2015.5.

71. Cerwenka A, Lanier LL. Natural killer cell memory in infection, inflammation and cancer. Nat Rev Immunol. 2016; 16:112-123. doi:10.1038/nri.2015.9.

72. Ljunggren HG, Kärre K. In search of the 'missing self': Mhc molecules and nk cell recognition. Immunol Today. 1990; 11:237-244. doi:10.1016/0167-5699(90)90097-s.

73. Sun JC, Lanier LL. Nk cell development, homeostasis and function: Parallels with cd8 $8^{+} \mathrm{t}$ cells. Nat Rev Immunol. 2011; 11:645-657. doi:10.1038/nri3044.

74. Meza Guzman LG, Keating N, Nicholson SE. Natural killer cells: Tumor surveillance and signaling. Cancers (Basel). 2020; 12. doi:10.3390/cancers12040952.

75. Orr MT, Lanier LL. Natural killer cell education and tolerance. Cell. 2010; 142:847-856. doi:https://doi.org/10.1016/j.cell.2010.08.031.

76. Boudreau JE, Hsu KC. Natural killer cell education and the response to infection and cancer therapy: Stay tuned. Trends Immunol. 2018; 39:222-239. doi:10.1016/j.it.2017.12.001.

77. Jonsson AH, Yokoyama WM. Natural killer cell tolerance licensing and other mechanisms. Adv Immunol. 2009; 101:27-79. doi:10.1016/s0065-2776(08)01002-x.

78. Tu MM, Mahmoud AB, Makrigiannis AP. Licensed and unlicensed nk cells: Differential roles in cancer and viral control. Front Immunol. 2016; 7:166. doi:10.3389/fimmu.2016.00166.

79. Brodin P, Höglund P. Beyond licensing and disarming: A quantitative view on nk-cell education. Eur $J$ Immunol. 2008; 38:2934-2937. doi:10.1002/eji.200838760. 
80. Thielens A, Vivier E, Romagné F. Nk cell mhc class i specific receptors (kir): From biology to clinical intervention. Curr Opin Immunol. 2012; 24:239-245. doi:10.1016/j.coi.2012.01.001.

81. Kim S, Poursine-Laurent J, Truscott SM, Lybarger L, Song Y-J, Yang L, et al. Licensing of natural killer cells by host major histocompatibility complex class i molecules. Nature. 2005; 436:709-713. doi:10.1038/nature03847.

82. He Y, Tian Z. Nk cell education via nonclassical mhc and non-mhc ligands. Cell Mol Immunol. 2017; 14:321-330. doi:10.1038/cmi.2016.26.

83. Crinier A, Narni-Mancinelli E, Ugolini S, Vivier E. Snapshot: Natural killer cells. Cell. 2020; 180:12801280.e1281. doi:10.1016/j.cell.2020.02.029.

84. Chiossone L, Dumas PY, Vienne M, Vivier E. Natural killer cells and other innate lymphoid cells in cancer. Nat Rev Immunol. 2018; 18:671-688. doi:10.1038/s41577-018-0061-z.

85. Vivier E, Tomasello E, Baratin M, Walzer T, Ugolini S. Functions of natural killer cells. Nat Immunol. 2008; 9:503-510. doi:10.1038/ni1582.

86. Zingoni A, Molfetta R, Fionda C, Soriani A, Paolini R, Cippitelli M, et al. Nkg2d and its ligands: "One for all, all for one". Front Immunol. 2018; 9:476. doi:10.3389/fimmu.2018.00476.

87. Shimasaki N, Jain A, Campana D. Nk cells for cancer immunotherapy. Nat Rev Drug Discov. 2020; $19: 200-$ 218. doi:10.1038/s41573-019-0052-1.

88. Naeimi Kararoudi M, Dolatshad H, Trikha P, Hussain SA, Elmas E, Foltz JA, et al. Generation of knockout primary and expanded human nk cells using cas9 ribonucleoproteins. J Vis Exp. 2018. doi: $10.3791 / 58237$.

89. O'Sullivan TE, Sun JC, Lanier LL. Natural killer cell memory. Immunity. 2015; 43:634-645. doi:10.1016/j.immuni.2015.09.013.

90. O'Leary JG, Goodarzi M, Drayton DL, von Andrian UH. T cell- and b cell-independent adaptive immunity mediated by natural killer cells. Nat Immunol. 2006; 7:507-516. doi:10.1038/ni1332.

91. Sheppard S, Sun JC. Virus-specific nk cell memory. J Exp Med. 2021; 218. doi:10.1084/jem.20201731.

92. Geary CD, Sun JC. Memory responses of natural killer cells. Semin Immunol. 2017; 31:11-19. doi:10.1016/j.smim.2017.08.012.

93. Fehniger TA, Cooper MA. Harnessing nk cell memory for cancer immunotherapy. Trends Immunol. 2016; 37:877-888. doi:10.1016/j.it.2016.09.005.

94. Leong JW, Chase JM, Romee R, Schneider SE, Sullivan RP, Cooper MA, et al. Preactivation with il-12, il15 , and il-18 induces cd25 and a functional high-affinity il-2 receptor on human cytokine-induced memorylike natural killer cells. Biol Blood Marrow Transplant. 2014; 20:463-473. doi:10.1016/j.bbmt.2014.01.006.

95. Cooper MA, Elliott JM, Keyel PA, Yang L, Carrero JA, Yokoyama WM. Cytokine-induced memory-like natural killer cells. Proc Natl Acad Sci U S A. 2009; 106:1915-1919. doi:10.1073/pnas.0813192106.

96. Pahl JHW, Cerwenka A, Ni J. Memory-like nk cells: Remembering a previous activation by cytokines and nk cell receptors. Front Immunol. 2018; 9:2796. doi:10.3389/fimmu.2018.02796.

97. Gang M, Marin ND, Wong P, Neal CC, Marsala L, Foster M, et al. Car-modified memory-like nk cells exhibit potent responses to nk-resistant lymphomas. Blood. 2020; 136:2308-2318. doi:10.1182/blood.2020006619.

98. Melaiu O, Lucarini V, Cifaldi L, Fruci D. Influence of the tumor microenvironment on nk cell function in solid tumors. Front Immunol. 2019; 10:3038. doi:10.3389/fimmu.2019.03038.

99. Hinshaw DC, Shevde LA. The tumor microenvironment innately modulates cancer progression. Cancer Res. 2019; 79:4557-4566. doi:10.1158/0008-5472.can-18-3962.

100. Binnewies M, Roberts EW, Kersten K, Chan V, Fearon DF, Merad M, et al. Understanding the tumor immune microenvironment (time) for effective therapy. Nat Med. 2018; 24:541-550. doi:10.1038/s41591018-0014-x.

101. Ni J, Wang X, Stojanovic A, Zhang Q, Wincher M, Bühler L, et al. Single-cell rna sequencing of tumorinfiltrating nk cells reveals that inhibition of transcription factor hif-1a unleashes nk cell activity. Immunity. 2020; 52:1075-1087.e1078. doi:10.1016/j.immuni.2020.05.001.

102. Degos C, Heinemann M, Barrou J, Boucherit N, Lambaudie E, Savina A, et al. Endometrial tumor microenvironment alters human nk cell recruitment, and resident $\mathrm{nk}$ cell phenotype and function. Front Immunol. 2019; 10:877. doi:10.3389/fimmu.2019.00877.

103. Harmon C, Robinson MW, Hand F, Almuaili D, Mentor K, Houlihan DD, et al. Lactate-mediated acidification of tumor microenvironment induces apoptosis of liver-resident nk cells in colorectal liver metastasis. Cancer Immunol Res. 2019; 7:335-346. doi:10.1158/2326-6066.cir-18-0481.

104. Nath PR, Pal-Nath D, Mandal A, Cam MC, Schwartz AL, Roberts DD. Natural killer cell recruitment and activation are regulated by cd47 expression in the tumor microenvironment. Cancer Immunol Res. 2019; 7:1547-1561. doi:10.1158/2326-6066.cir-18-0367.

105. Linden SK, Sutton P, Karlsson NG, Korolik V, McGuckin MA. Mucins in the mucosal barrier to infection. Mucosal Immunology. 2008; 1:183-197. doi:10.1038/mi.2008.5.

106. Taylor-Papadimitriou J, Burchell JM, Graham R, Beatson R. Latest developments in muc1 immunotherapy. Biochem. Soc. Trans. 2018; 46:659-668. doi:10.1042/BST20170400.

107. Ju T, Lanneau GS, Gautam T, Wang Y, Xia B, Stowell SR, et al. Human tumor antigens th and sialyl tn arise from mutations in cosmc. Cancer Res. 2008; 68:1636-1646. doi:10.1158/0008-5472.can-07-2345. 


\section{0 | Chapter 1}

108. Heublein S, Page S, Mayr D, Schmoeckel E, Trillsch F, Marmé F, et al. Potential interplay of the gatipotuzumab epitope ta-muc1 and estrogen receptors in ovarian cancer. Int J Mol Sci. 2019; 20. doi:10.3390/ijms20020295.

109. You F, Jiang L, Zhang B, Lu Q, Zhou Q, Liao X, et al. Phase 1 clinical trial demonstrated that muc1 positive metastatic seminal vesicle cancer can be effectively eradicated by modified anti-muc1 chimeric antigen receptor transduced t cells. Sci China Life Sci. 2016; 59:386-397. doi:10.1007/s11427-016-5024-7.

110. Ibrahim NK, Yariz KO, Bondarenko I, Manikhas A, Semiglazov V, Alyasova A, et al. Randomized phase ii trial of letrozole plus anti-muc1 antibody as 1402 in hormone receptor-positive locally advanced or metastatic breast cancer. Clin Cancer Res. 2011; 17:6822-6830. doi:10.1158/1078-0432.ccr-11-1151.

111. Altschuler Y, Kinlough CL, Poland PA, Bruns JB, Apodaca G, Weisz OA, et al. Clathrin-mediated endocytosis of muc1 is modulated by its glycosylation state. Molecular biology of the cell. 2000; 11:819831. doi:10.1091/mbc.11.3.819.

112. Finkelshtein D, Werman A, Novick D, Barak S, Rubinstein M. Ldl receptor and its family members serve as the cellular receptors for vesicular stomatitis virus. Proc Natl Acad Sci U S A. 2013; 110:7306-7311

113. Borst $\mathrm{L}$, van der Burg SH, van Hall $\mathrm{T}$. The nkg2a - hla-e axis as a novel checkpoint in the tumor microenvironment. Clin Cancer Res. 2020; 26:5549-5556. doi:10.1158/1078-0432.ccr-19-2095. 


\section{Chapter 2}

Defucosylation of tumor-specific humanized anti-MUC1 monoclonal antibody enhances NK cell-mediated antitumor cell cytotoxicity

Gong Y, Klein Wolterink RGJ, Gulaia V, Cloosen S, Ehlers FAI, Wieten L, Graus YF, Bos GMJ, Germeraad WTV. Cancers 2021, 13, 2579, doi:10.3390/cancers13112579. 


\section{Abstract}

Antibodies are commonly used in cancer immunotherapy because of their high specificity for tumor-associated antigens. Binding of antibodies can have direct effects on tumor cells, but also engages Natural Killer (NK) cells via their Fc receptor. Mucin 1 (MUC1) is a highly glycosylated protein expressed in normal epithelial cells, while the underglycosylated MUC1 epitope (MUC1-Tn/STn) is only expressed on malignant cells, making it an interesting diagnostic and therapeutic target. Several anti-MUC1 antibodies have been tested for therapeutic applications in solid tumors thus far without clinical success. Here, we describe the generation of fully humanized antibodies based on the murine 5E5 antibody, targeting the tumor-specific MUC1-Tn/STn epitope. We confirmed that these antibodies specifically recognize tumor-associated MUC1 epitopes and can activate human NK cells in vitro. Defucosylation of these newly developed anti-MUC1 antibodies further enhanced antigen-dependent cellular cytotoxicity (ADCC) mediated by NK cells. We show that endocytosis inhibitors augment the availability of MUC1Tn/STn epitopes on tumor cells, but do not further enhance ADCC in NK cells. Collectively, this study describes novel fully humanized anti-MUC1 antibodies that, especially after defucosylation, are promising therapeutic candidates for cellular immunotherapy.

Keywords: Antibody therapy; Natural Killer cells; MUC1; Antibody-dependent cellular cytotoxicity; breast cancer. 


\section{Introduction}

Cancer is still one of the leading causes of death around the globe. One hallmark of this neoplastic disease is avoidance of immune destruction. In hematologic malignancies, the tumor cells are dispersed in the blood, bone marrow and lymph nodes, which in principle facilitates access to tumors by immune cells to conduct eradication [1]. However, both in solid and hematological tumors, malignant cells aggregate and cultivate in a suppressive micro-environment, including hypoxia, low pH and inhibitory cytokines and molecules, which is detrimental for the function of immune cells [2]. Furthermore, malignant cells induce immune dysregulation by down-regulation of ligands that are natural activators of immune cells [3]. Therefore, tumor immunotherapy comprises various techniques that aim to enhance intrinsic immune mechanisms to promote eradication of tumor cells. Given the remarkable progress made with tumor immunotherapy, it was recently identified as a major breakthrough in clinical cancer treatment [2].

One of the most common forms of immunotherapy is the use of monoclonal antibodies (mAbs), designed to precisely influence the host response to tumor cells [4]. This treatment has a high specificity for tumor cells and thus has little or no side effects on the normal tissue [5]. The functional part of $\mathrm{mAbs}$ includes the antigen binding $\mathrm{F}(\mathrm{ab}) 2^{\prime}$ part and an Fc region. The antigen-binding fragment determines the specificity of mAb. The Fc portion of the $\mathrm{mAb}$ will bind on the Fc receptor expressed on the effector immune cells, including natural killer (NK) cells, macrophages, and dendritic cells [6].

NK cells belong to a group of innate immune cells that make up around $10 \%$ of the peripheral blood mononuclear cells (PBMC). NK cells are sentinels for "missing-self" cells and are able to distinguish virally infected cells and tumor cells from healthy counterparts via germline-encoded activating and inhibitory receptors. The activating receptors expressed on NK cells can identify appropriate ligands on virally infected, tumor, senescent and stressed cells [7]. These ligands will deliver an activating signal to NK cells, thereby initiating cytotoxic processes [8]. In contrast, the inhibitory receptors suppress NK cell activation and subsequently prevent killing of normal, healthy cells through recognition of self-proteins such as the widely-expressed major histocompatibility complex class I molecules (MHC-I) [9]. Moreover, human NK cells express the FcyRIIla receptor (also known as $\mathrm{CD} 16 \mathrm{6}$ ), which recognizes the Fc fragment of IgG. This induces NK cell-mediated eradication of IgG-opsonized abnormal cells via the secretion of their cytotoxic granules in a process called antibody-dependent cellular cytotoxicity (ADCC) [6]. Modulation of glycosylation of the antibody's Fc tail influences the interaction with the FcyRIIla receptor and can modulate this NK cell effector function. [10] More specifically, removal of fucose on the $\mathrm{N}$-glycan of the $\mathrm{Fc}$ tail has been shown to increase Fc binding affinity to the FcyRIIl $\alpha$, leading to enhanced ADCC [11,12].

Mucin1 (MUC1) is a highly glycosylated transmembrane protein expressed on the apical side of the cell membrane, which plays a paramount role in the protection and lubrication of normal epithelial cells [13]. In normal cells, the peptide core in heavily glycosylated MUC1 is masked by the O-glycan moieties that protect MUC1 from proteolytic cleavage enzymes. In addition, the glycosylation of MUC1 also stabilizes mucins on the apical side of the membrane by interfering with clathrin-mediated endocytosis. Cancer-related MUC1 proteins have shorter and less dense O-glycan side 
chains, resulting in exposure of the core domains of the protein on the cell surface. The MUC1 underglycosylation results in exposure of the epitopes MUC1-Tn and MUC1-STn to the immune system. This feature allows for the design of antibodies that can distinguish between tumor and normal cells [14]. Therefore, MUC1 has been investigated as a promising target towards solid tumors and even placed second as most favorable targets in immunotherapy [15]. Numerous groups have developed monoclonal antibodies against MUC1 both for diagnostic and therapeutic applications [16]. One example is the murine 5E5 antibody that recognizes MUC1-Tn and MUC1-STn epitopes [17]. Several groups have also successfully generated promising chimeric antigen receptor $T$ cells (CAR-T) based on the various murine mAb antibodies sequences [18]. Furthermore, MUC1 conserved peptide vaccine or mRNA-pulsed dendritic cells have also been used to induce immune responses against MUC1-positive tumors [19]. However, clinical studies using MUC1 antibodies have so far not resulted in the identification of a potent anti-MUC1 antibody that can be used for cancer treatment. Therefore, we hypothesized that a humanized version of a cancer-specific murine antiMUC1 antibody can be used to activate NK cells for cancer immunotherapy. We also asked if defucosylation of the Fc tail of this antibody further enhanced anti-tumor responses.

In this study, we explored the function of the humanized $5 E 5 \mathrm{mAb}$ in its natural $\lg \mathrm{G}$ configuration and compared it to the defucosylated IgG, aiming to increase antibodydependent cellular cytotoxicity by NK cells. We found that humanized anti-MUC1 antibodies indeed promoted ADCC and that defucosylated antibodies performed better. As the murine $5 \mathrm{E} 5$ antibody has been demonstrated as an attractive mAb candidate [20], humanized, defucosylated $5 \mathrm{E} 5$ could become a potential therapeutic $\mathrm{mAb}$ in many epithelial cancers. 


\section{Materials and Methods}

\subsection{Antibodies}

The original murine anti-MUC1 monoclonal antibody 5E5 (murine IgG1, binding MUC1 Tn/STn epitopes) was kindly provided by Professor Henrik Clausen (University of Copenhagen, Denmark). In addition, 214D4 (murine IgG1; binding pan-MUC1 epitopes) was kindly provided by Dr. John Hilkens (The Netherlands Cancer Institute, Amsterdam, The Netherlands).

CIM301-1 mAb is a recombinant fully human IgG1 comprising the 5E5 humanized $\mathrm{VH}$ and $\mathrm{VL}$ amino acid sequence. These human sequences were determined by investigators affiliated to the Cancer Research Technology Ltd. (London, UK) and were obtained under a research license by CiMaas. The whole corresponding coding sequence was first designed in silico; subsequent DNA was synthesized by Eurogentec (Liège, Belgium); and the cloning of the appropriate expression vectors was performed at GeneArt (Thermo Fisher Scientific, Regensburg, Germany). CIM301-4 (anti-HIV gp120) is a recombinant human IgG1 recognizing an epitope overlapping the CD4binding site of gp120. CIM301-1 and CIM301-4 mAb were produced in CHO-K1 (GeneArt). CIM301-8 is a recombinant human IgG1 comprising the same 5E5 humanized $\mathrm{VH}$ and $\mathrm{VL}$ amino acid sequence as CIM301-1 but with an ADCC optimized non-core-fucosylated Fc-domain based on Lonza Potelligent ${ }^{\circledR}$ technology produced transiently in CHO-K1SV (Lonza, Basel, Switzerland) on a research license under the Biowa technology to CiMaas. Antibodies used in this study are summarized in Table 1, and their structures are depicted in Figure $1 \mathrm{~A}$.

Table 1. Properties of anti-MUC1 antibodies

\begin{tabular}{llll}
\hline Name & Description & Isotype & Target \\
\hline 5E5 & Mouse antibody anti MUC1-Tn epitope & Murine IgG1 & Muc1-Tn/STn \\
\hline 214D4 & $\begin{array}{l}\text { Mouse antibody anti pan-MUC1 } \\
\text { epitope }\end{array}$ & Murine IgG & Pan-Muc1 \\
\hline CIM301-4 & Anti HIV-gp120 control antibody & Human IgG1 & gp120 \\
\hline CIM301-1 & Humanized 5E5 anti MUC1 & Human IgG1 & Muc1-(S)Tn \\
\hline CIM301-8 & $\begin{array}{l}\text { Defucosylated humanized 5E5 anti } \\
\text { MUC1 }\end{array}$ & Human IgG1 & Muc1-(S)Tn \\
\hline
\end{tabular}




\subsection{Cell lines and cell cultures}

$\mathrm{CHO}$ IdID cells were transfected with the coding sequence of MUC1 protein to produce $\mathrm{CHO}$ IdID MUC1 cells. Cells were maintained in IMDM medium (Thermo Fisher Scientific, Waltham, MA, USA) with 10\% FCS supplement with Gentamicin 418 (Thermo Fisher Scientific) at a concentration of $0.5 \mu \mathrm{g} / \mathrm{mL}$. To induce the MUC1-Tn epitope on CHO- IdID-MUC1 cells, $1 \mu \mathrm{M}$ of n-acetylgalactoseamine (GalNAc) (Sigma-Aldrich, Munich, Germany) was added to the medium, as described previously [21]. T-47D cells (HTB-133, ATCC, Manassas, VA, USA) were maintained in RPMI1640 (Thermo Fisher Scientific) supplemented with 10\% FCS (Greiner Bio-One, Frickenhausen, Germany), 1\% penicillin-streptomycin (Thermo Fisher Scientific, Waltham, MA, USA) and $0.2 \mathrm{U} / \mathrm{mL}$ bovine insulin (Sigma, Munich, Germany). MCF7 cells (HTB-22, ATCC) were cultured in EMEM (ATCC) with 10\% FCS, 1\% penicillin-streptomycin and $10 \mu \mathrm{g} / \mathrm{mL}$ human recombinant insulin (Sigma). SK-BR-3 (ACC 736, DSMZ, Braunschweig, Germany) cells were cultured in McCoy's 5A (Thermo Fisher Scientific) culture medium (Invitrogen, MT, USA), supplemented with $20 \%$ FCS and $1 \%$ penicillin-streptomycin. K-562 cells (CCL243, ATCC) were maintained in IMDM medium (Thermo Fisher Scientific) with 10\% FCS and $1 \%$ penicillin-streptomycin. Jurkat cells (ACC 282, DSMZ) were cultured in RPMI 1640 medium with Glutamax (Thermo Fisher Scientific), 10\% FCS and 1\% penicillinstreptomycin. All these cell lines were purchased as indicated between parenthesis followed by the generation of master cell and working cell banks. Cells were used from the working cell banks up to passage 10 .

\subsection{Human NK cell isolation and activation}

Primary human NK cells were isolated from anonymous buffy coats (Sanquin, Maastricht, The Netherlands). The use of buffy coats, being a by-product of a required Medical Ethical Review Committee (METC) procedure, does not need ethical approval in The Netherlands under the Dutch Code for Proper Secondary Use of Human Tissue. NK cells were isolated by negative selection with an NK cell isolation kit (Miltenyi Biotec, Bergisch Gladbach, Germany) using MACS beads as previously described [22]. Average purities were $>95 \%$. NK cells were cultured in RPMI-1640 medium (Thermo Fisher Scientific) supplemented with $10 \% \mathrm{FCS}, 1 \%$ penicillin-streptomycin and $1000 \mathrm{IU} / \mathrm{mL}$ recombinant human IL-2 (Proleukin, Novartis, Basel, Switzerland).

\subsection{Flow cytometry}

For flow cytometric analysis, cells were harvested, washed with PBS (Sigma) and first stained with Live/Dead Fixable Aqua Dead Cell Stain Kit (Thermo Fisher Scientific) in PBS on ice for $30 \mathrm{~min}$. Then $0.5 \times 10^{6} \mathrm{CHO}$ cells and tumor cells were resuspended in $100 \mu \mathrm{L}$ PBS and stained with $1 \mu \mathrm{g} / \mathrm{mL}$ murine $\mathrm{mAb}$ (5E5 or $214 \mathrm{D} 4$ ) or $1 \mu \mathrm{g} / \mathrm{mL}$ (or other concentrations, as indicated) humanized mAb (CIM301-4, CIM301-1 and CIM3018). After washing twice with PBS, cells were stained with secondary antibodies. For murine $\mathrm{mAb}$, primary antibodies were detected with $0.5 \mu \mathrm{g} / \mathrm{mL}$ AlexaFluor 647conjugated goat anti-mouse IgG $(\mathrm{H}+\mathrm{L})$ (Jackson Immuno Research, Cambridgeshire, United Kingdom). CIM301-4, CIM301-1 and CIM301-8 mAbs were detected with 0.5 $\mu \mathrm{g} / \mathrm{mL}$ AlexaFluor 647-conjugated goat anti-human IgG $(\mathrm{H}+\mathrm{L})$ (Jackson Immuno Research). Cells were washed twice with PBS after a 15 min incubation with secondary 
mAb. Cell pellets were resuspended in $200 \mu \mathrm{L}$ PBS for flow cytometric analysis. Fluorescence was read on a Canto II flow cytometer (BD Biosciences, San Jose, CA, USA). Data was analyzed with FlowJo 10.7 (TreeStar, Ashland, OR, USA) software.

\section{$2.5 \mathrm{NK}$ cell degranulation assay}

To evaluate NK cell activation by tumor cells or mAbs, CD107a expression on NK cells was analyzed using flow cytometry as previously described [22]. VioBlue-labeled anti-CD107a (clone H4A3, Miltenyi Biotec) or the corresponding isotype was added to the wells immediately after combining $10^{\wedge} 5 \mathrm{NK}$ cells with $10^{\wedge} 5$ cancer cells with or without the various humanized anti-MUC1 mAbs in a 96-well plate. After 1 hour of incubation at $37^{\circ} \mathrm{C}$ in humidified air containing $5 \% \mathrm{CO}_{2}, 10 \mu \mathrm{g} / \mathrm{mL}$ Brefeldin A (BFA, BD Bioscience) was added to each well. After 3 hours of further incubation, plates were put on ice and washed with PBS. After centrifugation, the supernatant was discarded and $50 \mu \mathrm{L}$ of antibody mix was added in each well: anti-CD3-FITC (SK7, Miltenyi Biotec), anti-CD56-PerCP-Vio770 (REA196, Miltenyi Biotec), anti-CD16-APC-H7 (3G8, BD).

\subsection{NK cell cytotoxicity (ADCC) assay}

NK cells isolated using negative selection as described above were used in cytotoxicity assays. T-47D and Jurkat cells were used as targets to investigate NK cell killing capacity. The antibodies CIM301-1 and CIM301-8 were added to evaluate their ability to enhance NK cell-mediated target cell killing. Target cells were labeled with Cell Tracker Deep Red Dye according to the manufacturer's protocol (Thermo Fisher Scientific) the night before the cytotoxicity assay. Tumor cells were harvested using trypsinization and washing, and were seeded at $2 \times 10^{4}$ cells per well in round-bottom 96-well plates. Then, NK cells were added at various effector : target (E:T) ratios. At the same time, different dilutions of anti-MUC1 antibodies were added. The total culture volume was $200 \mu \mathrm{L}$ per well. After 30 min preincubation with mAb, NK cells were added at various effector : target $(E: T)$ ratios. After $4 \mathrm{~h}$ of incubation, cells were put on ice and stained with Live/Dead Fixable Aqua (LDA) Dead Cell Stain Kit (Thermo Fisher Scientific). The percentage of specific killing was calculated using the following formula:

$\%$ specific killing

$$
=\frac{\% \text { LDA positive target cells }-\% \text { spontaneus } L D A \text { positive target cells }}{\% \text { vital cells }} \times 100
$$

\subsection{Endocytosis inhibitors in degranulation and cytotoxicity assays}

Prochlorperazine (PCZ, Sigma) and Dyngo4A (Abcam, Cambridge, UK) were resuspended in $0.1 \%(\mathrm{v} / \mathrm{v})$ DMSO (Sigma), which was also used as a solvent control. Degranulation and cytotoxicity assays were performed as described above, with addition 


\section{8 | Chapter 2}

of endocytosis inhibitors to the co-culture during the last hour of the assays in concentrations of $5 \mu \mathrm{M} \mathrm{PCZ}$ and $30 \mu \mathrm{M}$ Dyngo4A.

\subsection{Statistical analyses}

All statistical tests used in this study were completed with GraphPad Prism 8 software (Graphpad Software, San Diego, CA, USA). The specific statistical tests used for each comparison are specifically annotated in the figure legends, respectively. 


\section{Results}

\subsection{Fully humanized anti-MUC1 antibodies specifically recognize tumor- associated MUC1 glyco-epitopes}

To further explore the therapeutic potential of anti-MUC1 antibodies for cancer immunotherapy, we generated fully humanized anti-MUC1 antibodies based on the 5E5 murine antibody (Figure 1A). The 5E5 antibody was previously shown to specifically recognize cancer-specific MUC1 epitopes (MUC1-Tn/STn) and elicits strong immune responses in mice [17]. In addition to a fully humanized 5E5 antibody (designated CIM301-1), we generated CIM301-8, a defucosylated variant of the antibody with the aim of optimizing antibody-dependent cellular cytotoxicity (ADCC). Lastly, we generated a control antibody, CIM301-4, directed against the non-relevant HIV-gp120 epitope (Table 1).

We first confirmed that, in agreement with our previous data, the murine anti-MUC1 antibodies 214D4 (pan-MUC1) and 5E5 (cancer-specific MUC1-Tn/STn) recognized the relevant MUC1-Tn/STn epitopes on CHO cells expressing MUC1 epitopes [21], while no binding was observed in the parental CHO IdID cell line lacking MUC1 epitopes (Figure 1B). Likewise, the newly generated humanized anti-MUC1 antibodies CIM301-1 and CIM301-8 specifically bound to $\mathrm{CHO}$ cell lines expressing MUC1-Tn/STn epitopes, while the control antibody CIM301-4 showed no binding (Figure 1C). Moreover, CIM301-1 and CIM301-8 antibodies showed highly preferential binding to $\mathrm{CHO}$ cell lines expressing cancer-related MUC1-Tn/STn epitopes over $\mathrm{CHO}$ IdID cell lines expressing nonmodified MUC1. Next, we analyzed whether the humanized anti-MUC1 antibodies also recognized MUC1 Tn epitopes expressed on cancer cell lines. Previous studies have shown that the Jurkat cell line strongly expresses MUC1 Tn antigens due to a mutation in the COSMC gene that interferes with protein glycosylation [23]. Indeed, compared with the MUC1 Tn epitope-negative K-562 cell line, murine (Figure 1D) and humanized (Figure 1E) anti-MUC1 Tn antibodies displayed high binding affinity to Jurkat cells. Along the same line, the MUC1-expressing breast cancer cell lines MCF7 and T-47D also showed strong staining with anti-MUC1 Tn antibodies, while the MUC1 Tn-negative breast cancer cell line SK-BR-3 was only recognized by pan-MUC1 antibodies (Figure 1D). Together, these results confirm that the humanized anti-MUC1 antibodies CIM3011 and CIM301-8 specifically recognize the cancer-associated MUC1 Tn epitope. Therefore, we explored the applicability of these antibodies for immunotherapy in combination with NK cells. 
A

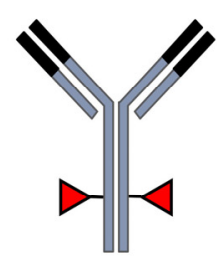

CIM301-4 anti-gp120 fucosylated

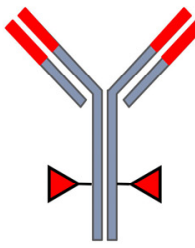

CIM301-1 anti-MUC1-Tn/STn

fucosylated

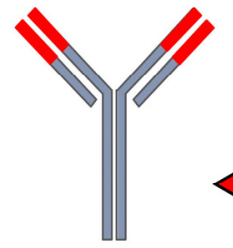

CIM301-8

anti-MUC1-Tn/STn

defucosylated

Fucose in $\mathrm{N}$-glycan in $\mathrm{Fc}$ region

B
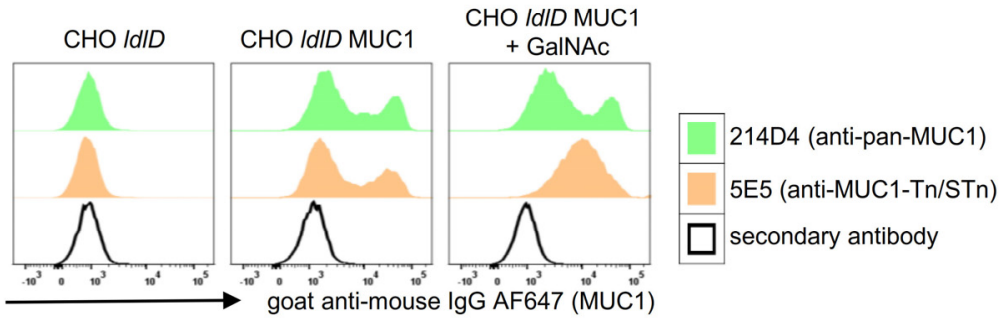

C
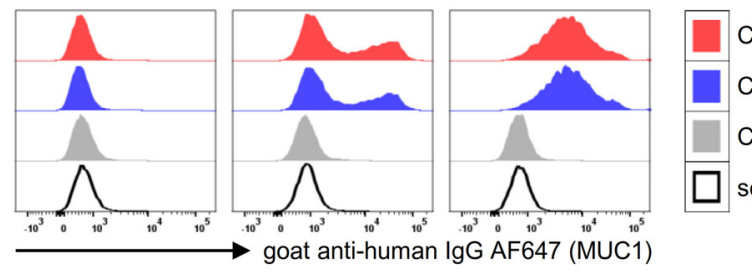

CIM301-8 (anti-MUC1-Tn)

CIM301-1 (anti-MUC1-Tn)

CIM301-4 (anti-gp120)

secondary antibody

D

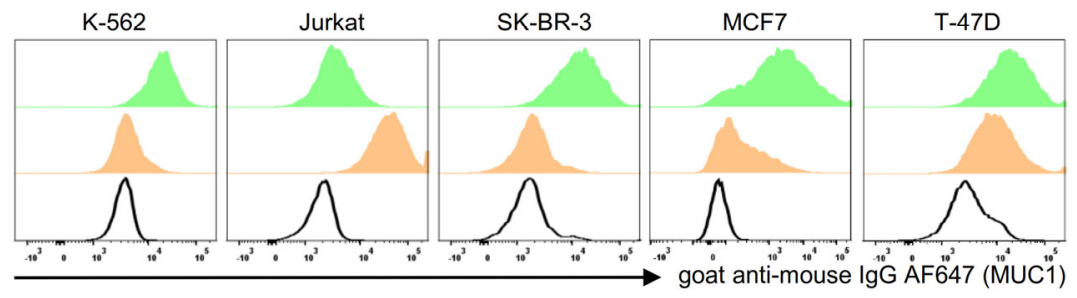

E

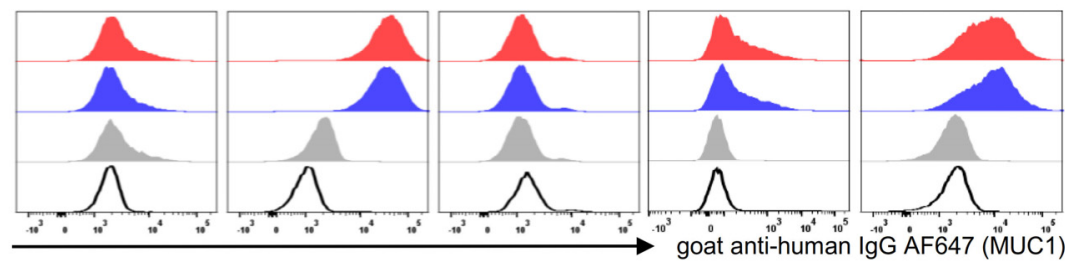

Figure 1. Fully humanized anti-MUC1 antibodies specifically recognize tumor-associated MUC1 epitopes. (A) Illustration outlining the design of the antibodies recognizing MUC1 or an irrelevant epitope (control) used in this study. Red triangles indicate glycosylation with fucose of the N-glycans in the antibody Fc tail. (B) Overlay histograms of MUC1 expression on $\mathrm{CHO}$ cell lines detected using murine antibodies. $\mathrm{CHO}$ cells expressed no MUC1 (CHO IdID), glycosylated MUC1 (CHO IdID MUC1) or MUC1-Tn/STn with tumorassociated glyco-epitopes (CHO IdID MUC1 + GalNAc). (C) Overlay histograms of MUC1 expression on $\mathrm{CHO}$ cell lines detected by humanized antibodies. $\mathrm{CHO}$ cell lines as described in B. (D) Expression levels of MUC1 or under-glycosylated MUC1 on cancer cell lines detected using 214D4 and 5E5 murine antibodies, respectively. (E) Histograms showing binding of humanized anti-MUC1 antibodies to various cancer cell lines. 


\subsection{Humanized anti-MUC1 antibodies conjugate NK cells and induce degranulation}

Monoclonal antibodies recognizing tumor antigens can induce ADCC through binding of the antibody Fc tail to the FcyRIlla (CD16) molecules on NK cells [6]. Therefore, we asked whether the humanized CIM-301 anti-MUC1 antibodies can indeed bind to NK cells (gating strategy in Figure S1A) by detecting labeled antibodies bound to the Fc portion of the anti-MUC1 antibody (Figure 2A). As expected, the murine anti-MUC1 antibodies did not bind to human NK cells (Figure 2B), while the fully humanized CIM301-1 and CIM301-8 antibodies showed robust binding to NK cells (Figure 2C). Notably, the defucosylated CIM301-8 displayed significantly stronger binding to FC receptors on NK cells (Figure 2C,D), confirming that removal of oligosaccharides in the Fc region of the antibody could be beneficial for ADCC in NK cells [6]. Activation of NK cells by crosslinking CD16 with antibodies induces strong activation in NK cells without the need for other activation signals [24]. NK cell activation leads to degranulation, which can be measured as CD107a expression levels. Therefore, we determined whether incubation of NK cells with humanized anti-MUC1 antibodies induced CD107a expression (Figure 2E, with gating strategy in Figure S1B). Indeed, we found that incubation of primary NK cells with humanized anti-MUC1 only (without tumor cells), could induce degranulation (Figure 2F, G). Compared with the CIM301-4 control antibody, the defucosylated anti-MUC1 antibody CIM301-8 showed higher induction of NK cell activation. Thus, humanized anti-MUC1 antibodies bind to the FcyRIlla receptor on human NK cells and can induce their activation, potentiating them to recognize and lyse MUC1-expressing tumor cells.

A

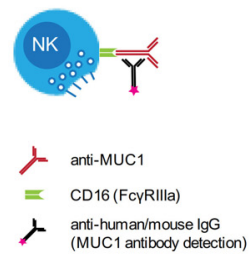

B

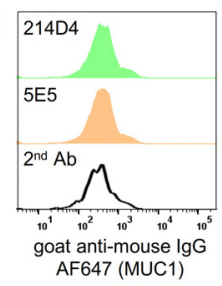

$\mathbf{F}$

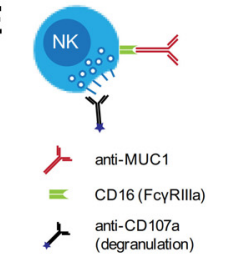

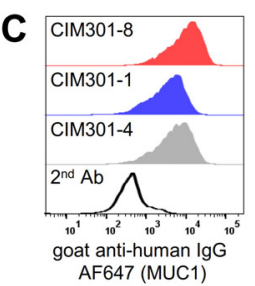

AF647 (MUC1)

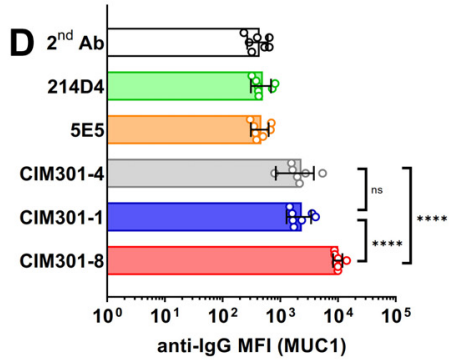

G

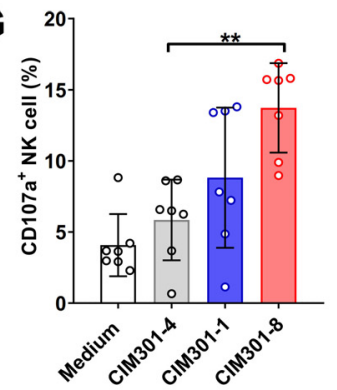

Figure 2. Humanized anti-MUC1 antibodies bind to CD16 on NK cells and induce degranulation. (A) Schematic overview outlining the detection of $\mathrm{mAb}$ binding via their Fc tail to the FcyRIIl $\alpha$ receptor (CD16) expressed on NK cells. (B) Binding of murine anti-MUC1 antibodies on primary human NK cells. Histograms show expression levels of the labeled anti-mouse IgG secondary antibodies used to detect MUC1 antibody 
binding. (C) Binding of humanized anti-MUC1 antibodies on primary NK cells. Experiment as in B, but using humanized anti-MUC1 antibodies, detected using anti-human IgG secondary antibodies. (D) Quantification of anti-MUC1 antibody binding to primary human NK cells. (E) Scheme depicting analysis of NK cell degranulation. (F) Flow cytometric analysis of NK cell degranulation following $4 \mathrm{~h}$ incubation of primary human NK cells with anti-MUC1 antibodies. One representative sample is shown with numbers indicating frequencies of cells within the indicated gate. (G) Quantification of the degranulation assay shown in F. Bars in D and G show mean \pm SD with individual data points as dots. Pooled data from 7 independent experiments with different donors performed at different time points. Statistical analysis using one-way ANOVA plus Tukey's multiple comparisons test. In $\mathbf{D}$, only biologically relevant comparisons were made. Not significant (n.s.); $\left.p<0.01{ }^{* *}\right)$; $p<0.0001{ }^{* * * *}$.

\subsection{Increasing concentrations of humanized anti-MUC1 antibodies enhance NK cell activation and induce CD16 down-regulation, but do not further enhance tumor cell killing}

Before comparing the standard humanized anti-MUC1 antibody to its defucosylated counterpart, we first determined the optimal antibody concentration for in vitro use. Monoclonal antibodies for cancer immunotherapy exert direct effects on the tumor cells, the effector NK cells and, on NK cell-mediated cytotoxicity. Therefore, we tested the defucosylated CIM301-8 antibody in all of these conditions. First, we incubated Jurkat tumor cells alone with increasing concentrations of humanized anti-MUC1 (CIM301-8) antibody and determined the level of saturation of the MUC1 epitopes and direct toxicity to tumor cells (Figure S2A). We observed that binding of MUC1 epitopes (Figure S2B and Figure S2C) and direct cytotoxic effects on the tumor cells (Figure S2D) were both dose-dependent. We then tested whether increasing antibody concentrations promoted antibody binding by NK cells and their degranulation (Figure 3A). Antibody-mediated NK cell activation via CD16 is a strong inducer of degranulation, but also induces downmodulation of CD16, most likely to prevent over activation of NK cells $[24,25]$. In line with these results, we observed that increasing doses of anti-MUC1 antibodies resulted in an increased fraction of degranulation NK cells (Figure 3B). In the presence of anti-MUC1 antibodies, the major proportion of these NK cells were negative for CD16 (Figure 3B and Figure S3A) and the overall CD16 levels decreased with increasing antibody concentrations (Figure 3C and Figure S3B). This suggested that the increase in degranulation of NK cells most likely came from cells that were activated by interaction of the antibody with CD16 leading to a loss in CD16 expression on these cells. Not surprisingly, these lower CD16 levels also resulted in lower levels of MUC1 antibodies bound to NK cells (Figure 3D and Figure S3C). Importantly, the observed enhanced NK cell degranulation came at the expense of higher NK cell death, almost doubling from $15 \%$ at $1 \mu \mathrm{g} / \mathrm{mL}$ to $26.7 \%$ at the highest concentration (Figure $3 \mathrm{E}$ ).

Lastly, we also titrated the antibody concentration in NK cell-Jurkat tumor cell coculture experiments, using degranulation and specific cytotoxicity as readout (Figure 4A). The addition of the anti-MUC1 antibody increased both degranulation and cytotoxicity against MUC1 Tn/STn+ Jurkat cells (Figure 4B and Figure 4C; white bars versus red bars). However, we did observe a dose-dependent effect of the antibody-induced degranulation of NK cells (Figure 4B and Figure 4C; shades of red), but this is not more than the increase that the direct effect of the antibody induced on the NK cells (Figure 3) Instead, the addition of extra NK cells (higher E:T ratios) was the main determinant of tumor cell killing (Figure 4C): in the presence of anti-MUC1 antibodies, the average tumor kill increased with on average $12.8 \%$ with every doubling of the E:T ratio. At the 
lowest E:T ratio tested $(0.25: 1)$, on average $21.1 \%$ of the tumor cells were killed, while at the highest $\mathrm{E}: \mathrm{T}$ ratio $(2: 1)$ this was $59.6 \%$.

In summary, the generated defucosylated humanized anti-MUC1 antibody dosedependently enhanced NK cell degranulation at the expense of CD16 down-modulation and increased NK cell death. NK cell-mediated responses against MUC1+ Jurkat tumor cells were importantly enhanced by the antibody, but antibody concentrations higher than $1 \mu \mathrm{g} / \mathrm{mL}$ showed no additional beneficial effects. Therefore, we concluded that 1 $\mu \mathrm{g} / \mathrm{mL}$ is the optimal concentration for further in vitro experiments.

A
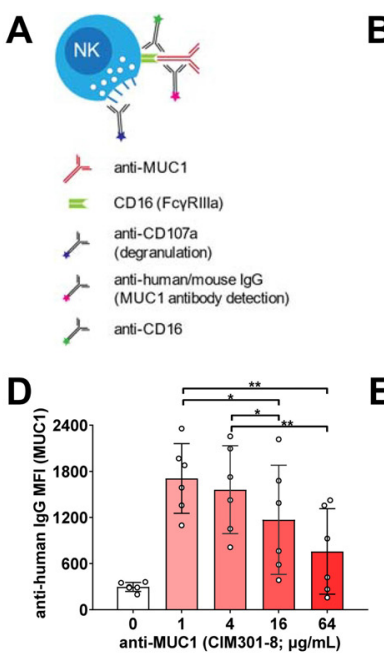

B
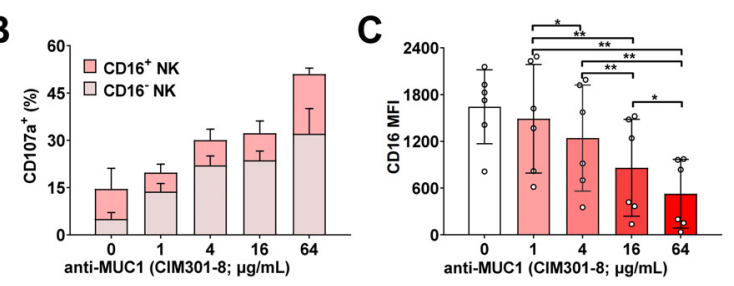

E

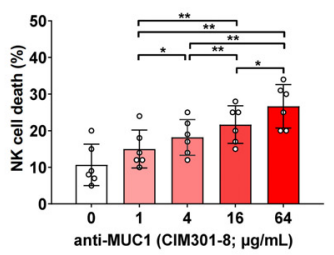

Figure 3. Increasing concentrations of humanized anti-MUC1 antibodies enhance degranulation and induce down-regulation of CD16 expression on NK cells. (A) Experimental setup for the detection of antiMUC1 antibody binding to NK cells, inducing degranulation and down-modulation of CD16 on NK cells. Human NK cells were incubated with increasing concentrations of anti-MUC1 (CIM301-8; defucosylated Fc-tail). Antibody binding to CD16 was analyzed using flow cytometric analysis of anti-human IgG antibodies. (B) Stacked bars show the fraction of NK cell degranulation for $\mathrm{CD}_{16}^{+}$and $\mathrm{CD}^{-} 6^{-} \mathrm{NK}$ populations in the presence of various antibody concentrations. (C) Quantification of CD16 expression on NK cells. (D) Quantification of anti-MUC1 antibody binding to the Fc tail of NK cells, shown as median fluorescence index (MFI) of anti-human IgG. (E) Quantification of NK cell death after $4 \mathrm{~h}$ incubation with increasing doses of anti-MUC1 antibody. Bars indicate mean $\pm S D$, dots are individual NK cell donors ( $n=6$ per experimental group). Statistical analysis using one-way ANOVA plus Tukey's multiple comparisons test. $p<0.05\left({ }^{*}\right) ; p<0.01\left({ }^{* *}\right)$.

A

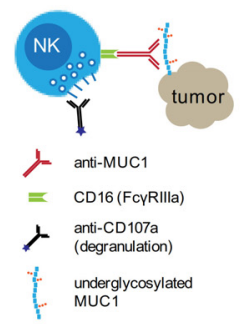

B

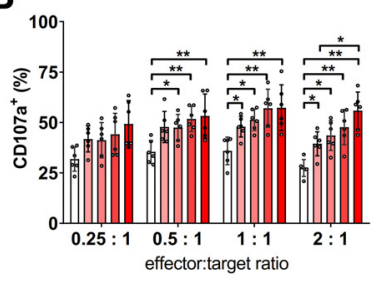

C

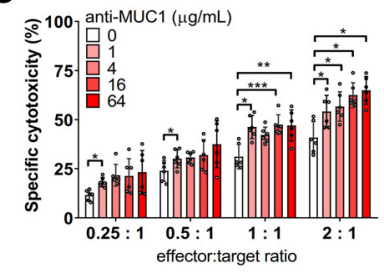

Figure 4. Increasing concentrations of humanized anti-MUC1 antibodies do not further enhance NK cell degranulation and cytotoxicity. (A) Experimental overview of the analysis of degranulation and cytotoxicity after co-culture of human NK cells with Jurkat MUC-Tn positive tumor cells in the presence of anti- 
MUC1 antibodies. (B) Fraction of degranulation NK cells after 4h of co-culture with different effector/tumor ratios and antibody concentrations. (C) Quantification of antibody-dependent cellular cytotoxicity at different $\mathrm{E}: \mathrm{T}$ ratios and antibody concentrations. Bars indicate mean $\pm \mathrm{SD}$, dots are individual NK cell donors from independent experiments ( $n=6$ per experimental group). Statistical analysis using two-way ANOVA plus Tukey's multiple comparisons test. Not significant (n.s.); $p<0.05\left(^{*}\right) ; p<0.01\left(^{* *}\right) ; p<0.001\left(^{* \star *}\right)$.

\subsection{Specific NK cell-mediated anti-tumor responses are enhanced by anti- MUC1 antibodies, especially after defucosylation}

Next, we asked whether defucosylation of these humanized anti-MUC1 antibodies show more potent anti-tumor responses. We compared the regular anti-MUC1 antibody CIM301-1 to the defucosylated CIM301-8 antibody in degranulation and cytotoxicity assays against two MUC1-Tn+ tumor cell lines (Figure 5A). First, we performed NK celltumor cell co-culture experiments and determined whether both antibodies could enhance the activation of NK cells. Indeed, CIM301-1 and CIM301-8 both enhanced NK cell degranulation in co-cultures with Jurkat cells and T-47D cells compared to the control antibody (CIM301-4) (Figure 5B, C). In ADCC assays, both anti-MUC1 antibodies enhanced cytotoxicity against Jurkat (Figure 5D and S4A) and T-47D cells (Figure 5E and S4B). Compared to the control antibody, the regular antibody increased anti-Jurkat cell responses by $23 \%$, while the defucosylated antibody increased cytotoxicity $49 \%$ (Figure 5D). For T-47D, the increases were $16 \%$ and $31 \%$, respectively (Figure 5E). These effects were most pronounced at higher E:T ratios and with CIM3018 antibody (Figure S4). In summary, these newly generated humanized anti-MUC1 antibodies importantly enhance NK cell-mediated cytotoxicity against different MUC1$\mathrm{Tn} / \mathrm{STn}$ epitope-positive tumor cells. In line with results obtained with other antibodies [20,26-28], defucosylation of anti-MUC1 antibodies further enhanced anti-tumor responses.

A
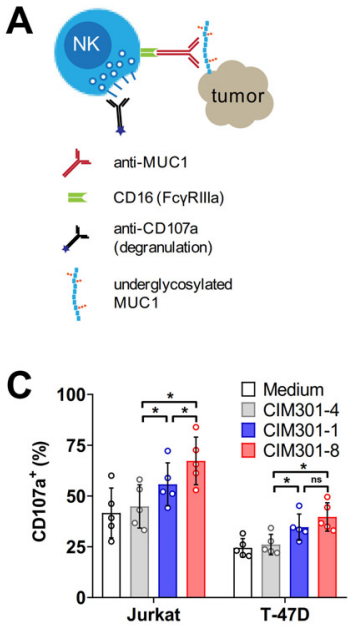

B

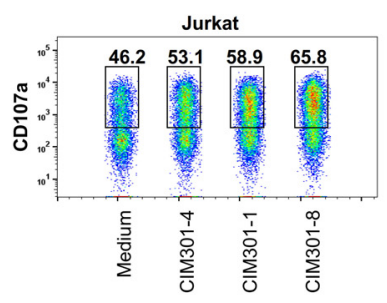

D

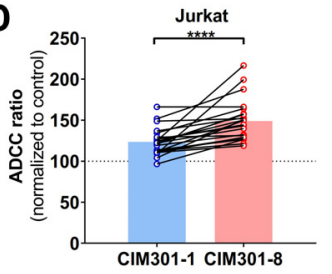

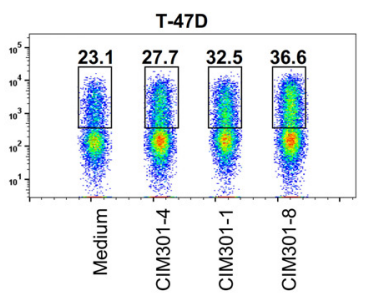

E

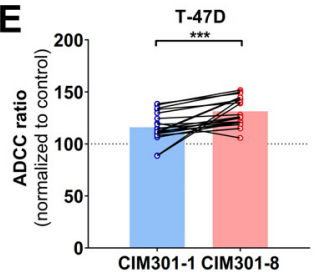

Figure 5. Defucosylation of humanized anti-MUC1 antibodies further enhances specific anti-tumor responses mediated by primary NK cells. (A) Scheme outlining the analysis of NK cell degranulation and cytotoxicity in co-cultures of primary human NK cells and MUC1 (S)Tn+ tumor cells in the presence of humanized anti-MUC1 antibodies. (B) Flow cytometric analysis of NK cell degranulation after $4 \mathrm{~h}$ co-culture in 
the presence of anti-MUC1 antibodies (regular CIM301-1 and defucosylated CIM301-8) or control antibodies (CIM301-4) at $1 \mu \mathrm{g} / \mathrm{mL}$. Per condition, one representative sample of CD56+ NK cells is shown, with gates and numbers indicating the fraction of degranulation NK cells. (C) Quantification of the degranulation assays described in B. Pooled data from 5 independent experiments performed with different NK cells donors at different time points. Differences between groups were tested using repeated measures one-way ANOVA with Šídák's multiple comparisons test. (D) Antibody-dependent cell-mediated cytotoxicity (ADCC) assay using human NK cells co-cultured with Jurkat tumor cells with or without $1 \mu \mathrm{g} / \mathrm{mL}$ regular (CIM301-1) or defucosylated (CIM301-8) antibodies. Dots represent individual data points from 6 independent experiments with 4 different effector: target ratios after normalization to the CIM301-4 control antibody. Dotted line indicates the CIM301-4 baseline level (100\%). (E) As D, but using T-47D tumor cells as target cells. Differences in ADCC (panels D and E) were calculated using repeated measures one-way ANOVA with Tukey's multiple comparisons test. P-values for comparisons to the control antibody for both antibodies using both tumor cells lines were $<0.0001$ (not shown). Not significant (n.s.); $p<0.05\left({ }^{*}\right) ; p<0.01\left({ }^{* *}\right) ; p<0.001\left({ }^{* * *}\right) ; p<0.0001$ $\left({ }^{* * * *}\right)$.

\subsection{The endocytosis inhibitor PCZ promotes tumor antigen expression, but does not enhance anti-tumor responses in NK cells}

Bioavailability of target antigens is an important determinant of the efficacy of antibody-mediated cancer therapy. MUC1 antigen expression on tumor cells is known to be dynamic, due to the internalization of the protein through clathrin- and dynamicmediated endocytosis [29]. This mechanism may facilitate the escape of tumor cells from NK cell-induced cell death by avoiding antibody opsonization [30]. Endocytosis inhibitors have been demonstrated to augment the ability of immune cells to eradicate EGFRpositive cells in both ex vivo and in vivo models [31]. Therefore, we hypothesized that the endocytosis inhibitors PCZ and Dyngo 4A could be used to increase the bioavailability of MUC1 antigens, thereby further promoting ADCC by NK cells. To test this, we investigated whether the addition of endocytosis inhibitors to tumor cell cultures promoted the expression of tumor-associated MUC1 epitopes (Figure 6A-6C). We found that, in the presence of PCZ, MUC1-Tn/STn epitope expression increased 2-fold on T47D cells (Figure 6B), but not on Jurkat cells (Figure S5A). However, Dyngo 4A [31], another endocytosis inhibitor did not alter the expression of MUC1 (Figure 6C and Figure S5B). Addition of endocytosis inhibitors to tumor cells in the presence of humanized anti-MUC1 antibodies had no negative effect on tumor cell viability (Figure 6D and Figure S5C). However, in co-culture experiments of NK cells and tumor cells (Figure 6E), endocytosis inhibitors neither enhanced NK cell degranulation (Figure 6F and Fig S5D) nor ADCC (Figure 6G and Figure S5E). Endocytosis inhibitors can thus promote the expression of tumor-associated MUC1 epitopes, but do not have beneficial effects on NK cell-mediated tumor elimination using anti-MUC1 antibodies. Importantly, we observed no negative effects of endocytosis inhibitors on NK cell viability (data not shown) and we do not observe negative effects of endocytosis inhibitors on cytotoxic capabilities of NK cells (Figure 6G). Alternatively, it is possible that in previous experiments we already attained the maximal stimulation with humanized anti-MUC1 antibodies. 
A

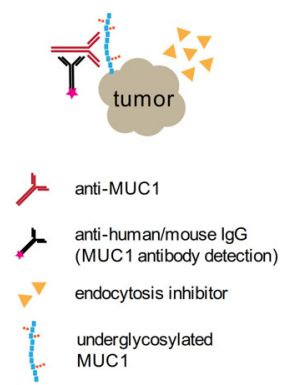

$\mathbf{E}$

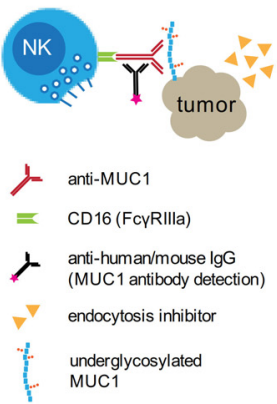

B

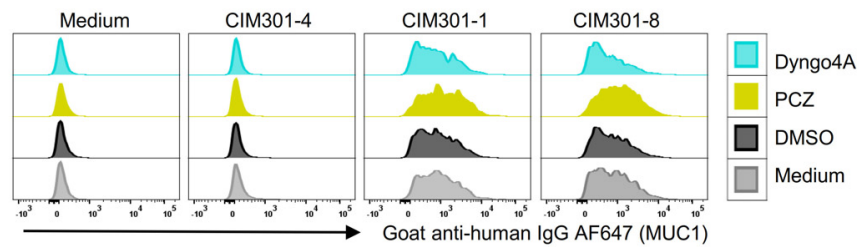

C

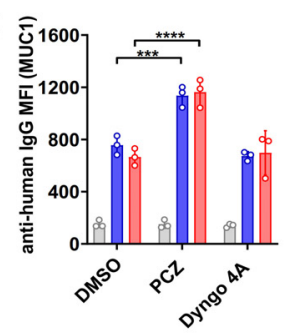

D 2007

$\mathbf{F}$
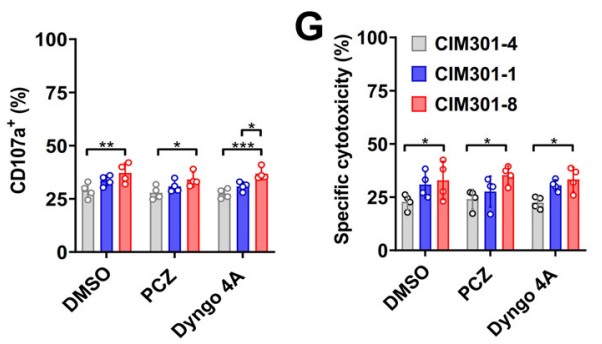

Figure 6. The endocytosis inhibitor PCZ promotes tumor antigen expression on T-47D cells, but does not enhance antibody-dependent NK cell-mediated cytotoxicity. (A) Experimental setup to determine whether endocytosis inhibitors enhance MUC1 epitope availability on tumor cells. (B) Overlay histograms of flow cytometric analysis of MUC1 epitope expression on T-47D tumor cells in the presence of endocytosis inhibitors using regular (CIM301-1) or defucosylated (CIM301-8) anti-MUC1 antibodies or an irrelevant control antibody (CIM301-4). Anti-human IgG antibodies were used to detect antibody binding on tumor cells. Endocytosis inhibitors were dissolved in DMSO, here used as a negative control. One representative sample is shown. (C) Flow cytometric quantification of MFI of MUC1 expression levels on T-47D cells after treatment with endocytosis inhibitors as described in B. Differences between control antibody (CIM301-4; grey bars) and anti-MUC1 antibodies (regular CIM301-1 in blue and defucosylated CIM301-8 in red) were all statistically significant with $p<0.0001$. (D) Viability of T-47D tumor cells after incubation with anti-MUC1 antibodies with or without endocytosis inhibitors. Pooled data from 3 independent experiments performed at different time-points. (E) Schematic overview of assays to test whether endocytosis inhibitors influence NK cell degranulation and cytotoxicity in the presence of anti-MUC1 antibodies. (F) Fraction of CD107a+ degranulation human NK cells in co-cultures with T-47D tumor cells at an effector/target ratio of 1:1 in the presence of anti-MUC1 antibodies and endocytosis inhibitors. NK cells and tumor cells were incubated for $4 \mathrm{~h}$, with endocytosis inhibitors $(5 \mu \mathrm{M}$ PCZ, $30 \mu \mathrm{M}$ Dyngo4A and 0.1\% (v/v) DMSO) added during the last hour. (G) Antibody-dependent NK-cell mediated cytotoxicity against T-47D tumor cells. Experimental setup as in $\mathbf{F}$. Panels $\mathbf{F}$ and $\mathbf{G}$ show pooled data from 4 independent experiments with different donors, performed at different time points. Differences between groups were determined using two-way ANOVA with Tukey's multiple comparisons test. Not significant (n.s.); $\left.p<0.05^{(*)} ; p<0.01{ }^{* *}\right) ; p<0.001\left(^{* * *}\right) ; p<0.0001\left(^{* * *}\right)$. 


\section{Discussion}

In this study, we investigated the potential of fully humanized anti-MUC1 antibodies based on the murine 5E5 antibody that specifically recognizes MUC1 Tn/STn cancerassociated epitopes. We demonstrated that the newly developed, fully human CIM3011 and CIM301-8 antibodies also functionally bind to MUC1-Tn/STn epitopes on tumor cell lines, and are capable of enhancing NK cell-mediated cytotoxicity upon binding to CD16. Throughout our analyses, we found that defucosylation of the Fc tail (CIM301-8) further enhanced anti-cancer effects. Together, these results are the next step in the use of these cancer-specific fully human anti-MUC1 antibodies for cancer immunotherapy, especially in the context of adoptive cell therapy.

The capacity of our humanized anti-MUC1 antibodies to enhance cytotoxicity was dependent on the NK cell quantity and on the fucosylation status of the antibody Fc region. In the pooled analysis of ADCC assays with Jurkat cells, fucosylated and defucosylated humanized anti-MUC1 antibodies enhanced cytotoxicity with $23 \%$ and $49 \%$ (Figure 5D), respectively. ADCC against the breast cancer cell line T-47D was enhanced with $31 \%$ by de defucosylated antibody CIM301-8 and 16\% with CIM301-1 (Figure 5E). These findings are in line with earlier studies investigating enhancement of ADCC by trastuzumab and cetuximab, both currently used in the clinical setting. For instance, the anti-HER2 antibody trastuzumab enhanced ADCC with $21 \%$ against the T47D cell line that expresses HER2 at low to moderate levels [32]. In a direct comparison between fucosylated and defucosylated cetuximab (anti-EGFR), PBMC-mediated ADCC was enhanced by $\sim 30 \%$ at various E:T ratios [33]. These findings should be confirmed in patient studies, as in the clinical setting, antibody availability, E:T ratios within the tumor microenvironment, other immune populations and various external factors will greatly impact on the antibody's anti-cancer efficacy.

MUC1 is an interesting target for antibody-based anti-cancer therapy, as it is expressed by a wide variety of tumors, including breast, ovarian, lung, colon, and pancreatic carcinomas as well as multiple myeloma [14]. MUC1 was identified almost 40 years ago, with cancer-specific aberrations in its expression pattern and glycosylation being recognized later [34]. The identification of the cancer-associated Tn and STn glycol forms of MUC1 also lead to the introduction of the murine 5E5 antibody [27]. There are several examples of MUC1 antibodies, such as HMFG1, HMFG2 [35] and SM3 [36], targeting a broad spectrum of MUC1 epitopes. These have been considered for cancer immunotherapy, but currently without much clinical success. For instance, a humanized form of HMFG1, designated AS1402 was tested in a phase II clinical study that was terminated early because of worse outcomes in patients receiving AS1402 [26]. HMFG2 and SM3 were demonstrated to be effective against MUC1-expressing tumor cell lines in mouse models, both as antibodies and as ScFv in the context of a CAR-T [37]. Additionally, gatipotuzumab (PankoMab) specifically reacts with cancer-associated MUC1 [28]. Although its safety was confirmed in a phase I trial (NCT01222624) [38], it did not show beneficial effects in a phase IIb trial in advanced ovarian cancer [39]. The 5E5 antibody under investigation in this study is of particular interest, because of its specificity for Tn/STn epitopes. It preferentially recognizes the $\mathrm{Tn}$ - and STn-carrying GSTA region of MUC1, while PankoMab also recognizes the less cancer-specific Tcarrying epitopes on the PDTR region $[17,27]$. Given the wide expression pattern of 
MUC1, the narrowed specificity of the 5E5 antibody is an advantage for any clinical application, as it reduces the risk of recognition of non-cancerous epitopes.

NK cells efficiently eliminate tumor cells and pathogens after binding the Fc portion of the monoclonal antibody, mainly through FcyRIIla (CD16a) [6]. Defucosylation of the Fc tail can be used to promote the binding to effector cells, and has shown encouraging results in in vitro experiments [40]. In line with this, we also found that the glycolengineered, defucosylated variant of the humanized anti-MUC1 antibody (CIM301-8) outperformed CIM301-1. Currently, three defucosylated antibodies are used for clinical care, and many more are being evaluated in clinical trials. Obinutuzumab is an antiCD20 defucosylated mAb approved by the FDA in 2013 and is used for the treatment of patients suffering from follicular lymphoma and CLL [41]. Compared to its fucosylated counterpart rituximab, the most widely used monoclonal antibody in the clinic, obinutuzumab enhances ADCC of NK cells in human lymphoma xenograft models and displays superior antitumor activity [42]. The other two defucosylated mAbs approved for clinical application are mogamulizumab, an anti-CCR4 antibody used for T cell lymphoma [43], and benralizumab, an anti-IL-5R antibody used for severe eosinophilic asthma [44]. In contrast to obinutuzumab, the latter two antibodies have not been directly compared to fucosylated antibodies. The previously mentioned anti-MUC1 antibody gatipotuzumab is defucosylated and has been tested in two clinical trials, so far without positive results [39]. Currently, another study investigates the combination of gatipotuzumab with tomuzotuximab (anti-EGFR) for treatment of patients with metastatic solid tumors (NCT03360734) [45]. In conclusion, antibody defucosylation should always be considered for cancer therapy, as it often enhances binding and clinical effects.

In addition to glycosylation of the Fc tail of an antibody $[6,40,46]$, there are several other determinants of the effectiveness of ADCC mediated by the interaction between NK cells and monoclonal antibodies: the affinity of the monoclonal antibody [47], stability of CD16 expression on NK cells [48], polymorphisms in CD16 [49] and concurrent Tolllike receptor agonists stimulation [50]. Macías-León et al. demonstrated that amino acids $\mathrm{H} 32, \mathrm{A33}, \mathrm{H} 35, \mathrm{H} 50, \mathrm{~S} 99, \mathrm{~T} 100$, and F102 in the heavy chain and $\mathrm{Y98}$ and $\mathrm{Y} 100$ in the light chain of the murine 5E5 antibody form the GalNAc-Tn epitope binding domain [51]. Sequence comparison of the humanized key antigen binding amino acids in the complementary determining regions (CDR)-3 show them to be identical to the 5E5 murine antibody [51]. As our humanized 5E5 antibodies bind the MUC1-Tn at the same level with murine 5E5 (Figure 1), we hypothesize that their affinities are similar. Surface plasmon resonance assays with MUC1-Tn peptides and the humanized and murine 5E5 antibodies may validate this hypothesis in the future.

Furthermore, we found that CD16 expression levels decreased with increasing antibody concentrations as reported previously [24,52,53]. This may seem counterproductive: in contrast to other activating receptors, antibody-mediated crosslinking of CD16 alone is sufficient to fully activate NK cells and to trigger degranulation [54]. However, it has been shown that shedding of CD16 is required to disassemble the established immune synapse between the NK cell and the tumor cell to allow for serial engagement of other targets [52]. Interestingly, in vitro studies have shown that NK cells can eliminate up to seven targets in 12 hours [55,56]. In addition, it has been suggested that CD16 down-regulation prevents NK cells from over activation and exhaustion [57]. Still, lower baseline levels of CD16 correlate with decreased ADCC responses in NK cells: compared with NK cells obtained from healthy donors, it was 
demonstrated that NK cells from cancer patients showed a significant reduction of both direct killing and ADCC against tumors, which was due to CD16 down-regulation [58]. In cancer patients, the expression levels of CD16, DNAM-1 and NKG2D have also been reported down-regulation on NK cells $[59,60]$.

A Western blot assay using NK cells after stimulation with tumor cells may further add proof to possible CD16 shedding. Most activating receptors on NK cells, such as NKG2D, can at least in part be rapidly recycled. Most activating receptors on NK cells, such as NKG2D, can at least in part be rapidly recycled. In contrast, down-modulation of CD16 is mediated by proteolytic cleavage by ADAM17 or MMP25 [24,25]. Consequently, recovery of CD16 expression may take days or weeks, as for instance reported after exposure to an influenza vaccine with partial recovery of CD16 expression only at day 18 [61]. Therefore, it is interesting to consider inhibition of proteolytic cleavage of CD16. Indeed, inhibition of the metalloprotease ADAM17 was shown to induce even stronger activation of NK cells [24]. In addition, inhibition of ADAM17 expression in NK cells, for instance using CRISPR/Cas9 or siRNA, could be used to prevent shedding of CD16 on NK cells used for cancer immunotherapy. In line with these results, our anti-MUC1 antibodies induced down-modulation of CD16 expression on NK cells in a dose-dependent manner. While we observed higher activation levels of NK cells with higher anti-MUC1 doses, we also found that NK cell-mediated ADCC responses were not enhanced at antibody concentrations higher than $1 \mu \mathrm{g} / \mathrm{mL}$. In addition to optimal dosing of antibodies and modulation of CD16 expression, clinical trials were recently launched using adoptive transfer of NK cells expressing the high affinity form of CD16 [48].

In this study, we also investigated whether endocytosis inhibitors can enhance the effects of anti-MUC1 antibodies by preventing the down regulation of MUC1 epitopes via endocytosis. MUC1 has been demonstrated to interact with the cell membrane through dynamic endocytosis, mediated by clathrin and dynamin [62]. The cell surface expression levels of underglycosylated, cancer-associated MUC1 are reduced compared with normal MUC1, either due to decreased delivery to the cell membrane or due to faster endocytosis, a critical tumor immune escape mechanism [29]. The process of endocytosis of MUC1 after binding of HMFG1 antibodies in MCF7 cells takes around 15 minutes [30]. Endocytosis inhibitors like PCZ and Dyngo 4A have been proven to unregulated tumor antigens such as EGFR and Her2, thereby enhancing ADCC [31]. In this study, we tested these two endocytosis inhibitors and found that MUC1-Tn/STn expression increased two-fold, though the effect was restricted to PCZ implemented on T-47D cells. There are various possible explanations for this phenomenon. For instance, different clathrin-independent pathways may be responsible for MUC1 internalization upon antibody [31]. Moreover, we found no changes in MUC1-Tn/STn epitope expression levels on Jurkat cells after treatment with either PCZ or Dyngo 4A. This could indicate that the Tn/STn epitope on Jurkat cell is so abundant that regular MUC1 internalization causes only slight changes on the cell surface, leaving the epitope expression pattern virtually unaltered. Thus, the exact mechanisms of MUC1 endocytosis in relation to ADCC need to be further studied to be able to allow anti-MUC1 antibodies to bind optimally to cancer-associated MUC1 epitopes. 


\section{Conclusion}

In this study we demonstrated that the 5E5-based, humanized anti-MUC1 antibodies CIM301-1 and CIM301-8 are potent enhancers of NK cell activation and cytotoxicity against MUC1-Tn/STn positive tumor cells in vitro. Defucosylation (CIM301-8) further potentiated the NK cell response. Hereafter in vivo studies and clinical trials should be conducted to explore the full therapeutic potential of these newly generated antibodies. Furthermore, the developed antibodies could also be tested in the context of adoptive cell therapy with effector cells redirected with chimeric antigen receptors (CAR) employing the ScFv of the humanized 5E5 antibodies. Given the cancer-specificity of these 5E5-based antibodies, combined with the fact that many different tumors show expression of cancerous MUC1 epitopes, CIM301-1 but especially CIM301-8 are interesting candidates for cancer immunotherapy.

\section{Acknowledgements}

We cordially appreciate Henrik Clausen (from University of Copenhagen, Denmark) for providing murine 5E5 antibody and John Hilkens (from The Netherlands Cancer Institute, Amsterdam, The Netherlands) for originally offering the 214D4 antibody to this study.

Y.G. thanks the China Scholarship Council (CSC) (No. 201707720056) for fellowship support. R.G.J.K.W. is supported by a Kootstra Talent Fellowship (Maastricht University) and a Marie Skłodowska-Curie Individual fellowship (799810-TOPNIN; European Union). The research was supported by Cancer Research Foundation Limburg (KOFL-2012-03) and GROW School of Maastricht University. 
1. Binnewies M, Roberts EW, Kersten K, Chan V, Fearon DF, Merad M, et al. Understanding the tumor immune microenvironment (time) for effective therapy. Nat Med. 2018; 24:541-550. doi:10.1038/s41591-018-0014-X.

2. Lei X, Lei Y, Li J-K, Du W-X, Li R-G, Yang J, et al. Immune cells within the tumor microenvironment: Biological functions and roles in cancer immunotherapy. Cancer Lett. 2020; 470:126-133. doi:https://doi.org/10.1016/j.canlet.2019.11.009.

3. Molfetta R, Zingoni A, Santoni A, Paolini R. Post-translational mechanisms regulating nk cell activating receptors and their ligands in cancer: Potential targets for therapeutic intervention. Front Immunol. 2019; 10:2557. doi:10.3389/fimmu.2019.02557.

4. Dixon KJ, Wu J, Walcheck B. Engineering anti-tumor monoclonal antibodies and fc receptors to enhance adcc by human nk cells. Cancers (Basel). 2021; 13:312. doi:10.3390/cancers13020312.

5. Singh S, Kumar NK, Dwiwedi P, Charan J, Kaur R, Sidhu P, et al. Monoclonal antibodies: A review. Curr Clin Pharmacol. 2018; 13:85-99. doi:10.2174/1574884712666170809124728.

6. Murin CD. Considerations of antibody geometric constraints on nk cell antibody dependent cellular cytotoxicity. Front Immunol. 2020; 11:1635. doi:10.3389/fimmu.2020.01635.

7. Dianat-Moghadam H, Mahari A, Heidarifard M, Parnianfard N, Pourmousavi-Kh L, Rahbarghazi R, et al. Nk cells-directed therapies target circulating tumor cells and metastasis. Cancer Lett. 2021; 497:41-53. doi:https://doi.org/10.1016/j.canlet.2020.09.021.

8. Guillerey C, Huntington ND, Smyth MJ. Targeting natural killer cells in cancer immunotherapy. Nat Immunol. 2016; 17:1025. doi:10.1038/ni.3518.

9. Huntington ND, Cursons J, Rautela J. The cancer-natural killer cell immunity cycle. Nat Rev Cancer. 2020; 20:437-454. doi:10.1038/s41568-020-0272-z.

10. van der Horst HJ, Nijhof IS, Mutis T, Chamuleau MED. Fc-engineered antibodies with enhanced fceffector function for the treatment of b-cell malignancies. Cancers (Basel). 2020; 12:3041. doi:10.3390/cancers12103041.

11. Shinkawa T, Nakamura K, Yamane N, Shoji-Hosaka E, Kanda Y, Sakurada M, et al. The absence of fucose but not the presence of galactose or bisecting n-acetylglucosamine of human igg1 complex-type oligosaccharides shows the critical role of enhancing antibody-dependent cellular cytotoxicity. J Biol Chem. 2003; 278:3466-3473. doi:10.1074/jbc.M210665200.

12. Shields RL, Lai J, Keck R, O'Connell LY, Hong K, Meng YG, et al. Lack of fucose on human igg1 nlinked oligosaccharide improves binding to human fcgamma riii and antibody-dependent cellular toxicity. J Biol Chem. 2002; 277:26733-26740. doi:10.1074/jbc.M202069200.

13. Linden SK, Sutton P, Karlsson NG, Korolik V, McGuckin MA. Mucins in the mucosal barrier to infection. Mucosal Immunology. 2008; 1:183-197. doi:10.1038/mi.2008.5.

14. Bhatia R, Gautam SK, Cannon A, Thompson C, Hall BR, Aithal A, et al. Cancer-associated mucins: Role in immune modulation and metastasis. Cancer Metastasis Rev. 2019; 38:223-236. doi:10.1007/s10555-018-09775-0.

15. Cheever MA, Allison JP, Ferris AS, Finn OJ, Hastings BM, Hecht TT, et al. The prioritization of cancer antigens: A national cancer institute pilot project for the acceleration of translational research. Clin Cancer Res. 2009; 15:5323-5337. doi:10.1158/1078-0432.ccr-09-0737.

16. Cloosen S, Arnold J, Thio M, Bos GM, Kyewski B, Germeraad WT. Expression of tumor-associated differentiation antigens, muc1 glycoforms and cea, in human thymic epithelial cells: Implications for self-tolerance and tumor therapy. Cancer Res. 2007; 67:3919-3926. doi:10.1158/0008-5472.can06-2112.

17. Sørensen AL, Reis CA, Tarp MA, Mandel U, Ramachandran K, Sankaranarayanan V, et al. Chemoenzymatically synthesized multimeric tn/stn muc1 glycopeptides elicit cancer-specific antimuc1 antibody responses and override tolerance. Glycobiology. 2006; 16:96-107. doi:10.1093/glycob/cwj044.

18. Posey AD, Schwab RD, Boesteanu AC, Steentoft C, Mandel U, Engels B, et al. Engineered car $t$ cells targeting the cancer-associated tn-glycoform of the membrane mucin muc1 control adenocarcinoma. Immunity. 2016; 44:1444-1454. doi:10.1016/j.immuni.2016.05.014.

19. Tarp MA, Clausen H. Mucin-type o-glycosylation and its potential use in drug and vaccine development. Biochim Biophys Acta. 2008; 1780:546-563. doi:10.1016/j.bbagen.2007.09.010.

20. Lavrsen K, Madsen CB, Rasch MG, Woetmann A, Ødum N, Mandel U, et al. Aberrantly glycosylated muc1 is expressed on the surface of breast cancer cells and a target for antibody-dependent cellmediated cytotoxicity. Glycoconjugate Journal. 2013; 30:227-236. doi:10.1007/s10719-012-9437-7. 
21. Van Elssen $\mathrm{CH}$, Clausen H, Germeraad WT, Bennet EP, Menheere PP, Bos GM, et al. Flow cytometry-based assay to evaluate human serum muc1-tn antibodies. J Immunol Methods. 2011; 365:87-94. doi:10.1016/j.jim.2010.12.006.

22. Gong Y, Klein Wolterink RGJ, Janssen I, Groot AJ, Bos GMJ, Germeraad WTV. Rosuvastatin enhances vsv-g lentiviral transduction of nk cells via upregulation of the low-density lipoprotein receptor. Mol Ther Methods Clin Dev. 2020; 17:634-646. doi:10.1016/j.omtm.2020.03.017.

23. Sun X, Ju T, Cummings RD. Differential expression of cosmc, t-synthase and mucins in tn-positive colorectal cancers. BMC Cancer. 2018; 18:827. doi:10.1186/s12885-018-4708-8.

24. Romee R, Foley B, Lenvik T, Wang Y, Zhang B, Ankarlo D, et al. Nk cell cd16 surface expression and function is regulated by a disintegrin and metalloprotease-17 (adam17). Blood. 2013; 121:35993608. doi:10.1182/blood-2012-04-425397.

25. Peruzzi G, Femnou L, Gil-Krzewska A, Borrego F, Weck J, Krzewski K, et al. Membrane-type 6 matrix metalloproteinase regulates the activation-induced downmodulation of cd16 in human primary nk cells. J Immunol. 2013; 191:1883-1894. doi:10.4049/jimmunol.1300313.

26. Ibrahim NK, Yariz KO, Bondarenko I, Manikhas A, Semiglazov V, Alyasova A, et al. Randomized phase ii trial of letrozole plus anti-muc1 antibody as1402 in hormone receptor-positive locally advanced or metastatic breast cancer. Clin Cancer Res. 2011; 17:6822-6830. doi:10.1158/10780432.ccr-11-1151.

27. Tarp MA, Sørensen AL, Mandel U, Paulsen H, Burchell J, Taylor-Papadimitriou J, et al. Identification of a novel cancer-specific immunodominant glycopeptide epitope in the muc1 tandem repeat. Glycobiology. 2007; 17:197-209. doi:10.1093/glycob/cwl061.

28. Danielczyk A, Stahn R, Faulstich D, Löffler A, Märten A, Karsten U, et al. Pankomab: A potent new generation anti-tumour muc1 antibody. Cancer Immunol Immunother. 2006; 55:1337-1347. doi:10.1007/s00262-006-0135-9.

29. Altschuler Y, Kinlough CL, Poland PA, Bruns JB, Apodaca G, Weisz OA, et al. Clathrin-mediated endocytosis of muc1 is modulated by its glycosylation state. Molecular biology of the cell. 2000; 11:819-831. doi:10.1091/mbc.11.3.819.

30. Pericleous LM, Richards J, Epenetos AA, Courtenay-Luck N, Deonarain MP. Characterisation and internalisation of recombinant humanised hmfg-1 antibodies against muc1. Br J Cancer. 2005; 93:1257-1266. doi:10.1038/sj.bjc.6602847.

31. Chew HY, De Lima PO, Gonzalez Cruz JL, Banushi B, Echejoh G, Hu L, et al. Endocytosis inhibition in humans to improve responses to adcc-mediating antibodies. Cell. 2020; 180:895-914.e827. doi:10.1016/j.cell.2020.02.019.

32. Collins DM, O'Donovan N, McGowan PM, O'Sullivan F, Duffy MJ, Crown J. Trastuzumab induces antibody-dependent cell-mediated cytotoxicity (adcc) in her-2-non-amplified breast cancer cell lines. Ann Oncol. 2012; 23:1788-1795. doi:https://doi.org/10.1093/annonc/mdr484.

33. Nakajima T, Okayama H, Ashizawa M, Noda M, Aoto K, Saito M, et al. Augmentation of antibodydependent cellular cytotoxicity with defucosylated monoclonal antibodies in patients with gi-tract cancer. Oncology letters. 2018; 15:2604-2610. doi:10.3892/ol.2017.7556.

34. Taylor-Papadimitriou J, Burchell JM, Graham R, Beatson R. Latest developments in muc1 immunotherapy. Biochem. Soc. Trans. 2018; 46:659-668. doi:10.1042/BST20170400.

35. Taylor-Papadimitriou J, Peterson JA, Arklie J, Burchell J, Ceriani RL, Bodmer WF. Monoclonal antibodies to epithelium-specific components of the human milk fat globule membrane: Production and reaction with cells in culture. Int J Cancer. 1981; 28:17-21. doi:10.1002/ijc.2910280104.

36. Burchell J, Gendler S, Taylor-Papadimitriou J, Girling A, Lewis A, Millis R, et al. Development and characterization of breast cancer reactive monoclonal antibodies directed to the core protein of the human milk mucin. Cancer Res. 1987; 47:5476-5482

37. Wilkie S, Picco G, Foster J, Davies DM, Julien S, Cooper L, et al. Retargeting of human t cells to tumor-associated muc1: The evolution of a chimeric antigen receptor. J Immunol. 2008; 180:49014909. doi:10.4049/jimmunol.180.7.4901.

38. Fiedler W, DeDosso S, Cresta S, Weidmann J, Tessari A, Salzberg M, et al. A phase i study of pankomab-gex, a humanised glyco-optimised monoclonal antibody to a novel tumour-specific muc1 glycopeptide epitope in patients with advanced carcinomas. Eur J Cancer. 2016; 63:55-63. doi:https://doi.org/10.1016/j.ejca.2016.05.003.

39. Ledermann J, Sehouli J, Zurawski B, Raspagliesi F, De Giorgi U, Banerjee S, et al. A double-blind, placebo-controlled, randomized, phase 2 study to evaluate the efficacy and safety of switch maintenance therapy with the anti-ta-muc1 antibody pankomab-gex after chemotherapy in patients with recurrent epithelial ovarian carcinoma. Ann Oncol. 2017; 28:v626. doi:10.1093/annonc/mdx440.035. 
40. Pereira NA, Chan KF, Lin PC, Song Z. The "less-is-more" in therapeutic antibodies: Afucosylated anti-cancer antibodies with enhanced antibody-dependent cellular cytotoxicity. mAbs. 2018; 10:693711. doi:10.1080/19420862.2018.1466767.

41. Marcus R, Davies A, Ando K, Klapper W, Opat S, Owen C, et al. Obinutuzumab for the first-line treatment of follicular lymphoma. N Engl J Med. 2017; 377:1331-1344. doi:10.1056/NEJMoa1614598.

42. Mössner E, Brünker P, Moser S, Püntener U, Schmidt C, Herter S, et al. Increasing the efficacy of cd20 antibody therapy through the engineering of a new type ii anti-cd20 antibody with enhanced direct and immune effector cell-mediated b-cell cytotoxicity. Blood. 2010; 115:4393-4402. doi:10.1182/blood-2009-06-225979.

43. Ishii T, Ishida T, Utsunomiya A, Inagaki A, Yano H, Komatsu H, et al. Defucosylated humanized anticcr4 monoclonal antibody kw-0761 as a novel immunotherapeutic agent for adult t-cell leukemia/lymphoma. Clin Cancer Res. 2010; 16:1520-1531. doi:10.1158/1078-0432.ccr-09-2697.

44. Kolbeck R, Kozhich A, Koike M, Peng L, Andersson CK, Damschroder MM, et al. Medi-563, a humanized anti-il-5 receptor a mab with enhanced antibody-dependent cell-mediated cytotoxicity

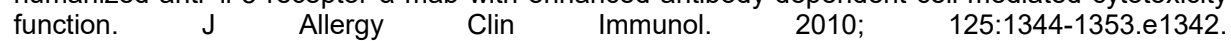
doi:https://doi.org/10.1016/j.jaci.2010.04.004.

45. Fiedler W, Cresta S, Schulze-Bergkamen H, De Dosso S, Weidmann J, Tessari A, et al. Phase i study of tomuzotuximab, a glycoengineered therapeutic antibody against the epidermal growth factor receptor, in patients with advanced carcinomas. ESMO Open. 2018; 3:e000303. doi:10.1136/esmoopen-2017-000303.

46. Zahavi D, AIDeghaither D, O'Connell A, Weiner LM. Enhancing antibody-dependent cell-mediated cytotoxicity: A strategy for improving antibody-based immunotherapy. Antibody Therapeutics. 2018; 1:7-12. doi:10.1093/abt/tby002.

47. Sharma P, Marada V, Cai Q, Kizerwetter M, He Y, Wolf SP, et al. Structure-guided engineering of the affinity and specificity of cars against tn-glycopeptides. Proc Natl Acad Sci U S A. 2020. doi:10.1073/pnas.1920662117.

48. Zhu H, Blum RH, Bjordahl R, Gaidarova S, Rogers P, Lee TT, et al. Pluripotent stem cell-derived nk cells with high-affinity noncleavable cd16a mediate improved antitumor activity. Blood. 2020; 135:399-410. doi:10.1182/blood.2019000621.

49. Rataj F, Jacobi SJ, Stoiber S, Asang F, Ogonek J, Tokarew N, et al. High-affinity cd16-polymorphism and fc-engineered antibodies enable activity of cd16-chimeric antigen receptor-modified $t$ cells for cancer therapy. Br J Cancer. 2018. doi:10.1038/s41416-018-0341-1.

50. Moreno M, Mol BM, Mensdorff-Pouilly Sv, Verheijen RHM, von Blomberg BME, van den Eertwegh AJM, et al. Toll-like receptor agonists and invariant natural killer t-cells enhance antibody-dependent $\begin{array}{llllll}\text { cell-mediated } \quad \text { cytotoxicity } \quad \text { (adcc). Cancer } & \text { Lett. 2008; }\end{array}$ doi:https://doi.org/10.1016/j.canlet.2008.06.028.

51. Macías-León J, Bermejo IA, Asín A, García-García A, Compañón I, Jiménez-Moreno E, et al. Structural characterization of an unprecedented lectin-like antitumoral anti-muc1 antibody. Chem Commun (Camb). 2020; 56:15137-15140. doi:10.1039/d0cc06349e.

52. Srpan K, Ambrose A, Karampatzakis A, Saeed M, Cartwright ANR, Guldevall K, et al. Shedding of cd16 disassembles the nk cell immune synapse and boosts serial engagement of target cells. J Cell Biol. 2018; 217:3267-3283. doi:10.1083/jcb.201712085.

53. Pham DH, Kim JS, Kim SK, Shin DJ, Uong NT, Hyun H, et al. Effects of adam10 and adam17 inhibitors on natural killer cell expansion and antibody-dependent cellular cytotoxicity against breast cancer cells in vitro. Anticancer Res. 2017; 37:5507-5513. doi:10.21873/anticanres.11981.

54. Bryceson YT, March ME, Barber DF, Ljunggren H-G, Long EO. Cytolytic granule polarization and degranulation controlled by different receptors in resting nk cells. J Exp Med. 2005; 202:1001-1012. doi:10.1084/jem.20051143.

55. Bhat R, Watzl C. Serial killing of tumor cells by human natural killer cells - enhancement by therapeutic antibodies. PLoS One. 2007; 2:e326. doi:10.1371/journal.pone.0000326.

56. Vanherberghen B, Olofsson PE, Forslund E, Sternberg-Simon M, Khorshidi MA, Pacouret S, et al. Classification of human natural killer cells based on migration behavior and cytotoxic response. Blood. 2013; 121:1326-1334. doi:10.1182/blood-2012-06-439851.

57. Gauthier M, Laroye C, Bensoussan D, Boura C, Decot V. Natural killer cells and monoclonal antibodies: Two partners for successful antibody dependent cytotoxicity against tumor cells. Critical Reviews in Oncology/Hematology. 2021; 160:103261. doi:https://doi.org/10.1016/j.critrevonc.2021.103261.

58. Kaur K, Safaie T, Ko MW, Wang Y, Jewett A. Adcc against mica/b is mediated against differentiated oral and pancreatic and not stem-like/poorly differentiated tumors by the nk cells; loss in cancer 


\section{4 | Chapter 2}

patients due to down-modulation of cd16 receptor. Cancers (Basel). 2021; 13. doi:10.3390/cancers13020239.

59. Pazina T, MacFarlane AWt, Bernabei L, Dulaimi E, Kotcher R, Yam C, et al. Alterations of nk cell phenotype in the disease course of multiple myeloma. Cancers (Basel). 2021; 13:226. doi:10.3390/cancers13020226.

60. Mantovani S, Varchetta S, Mele D, Donadon M, Torzilli G, Soldani C, et al. An anti-mica/b antibody and il-15 rescue altered $n k g 2 d-d e p e n d e n t ~ n k$ cell responses in hepatocellular carcinoma. Cancers (Basel). 2020; 12:3583. doi:10.3390/cancers12123583.

61. Goodier MR, Lusa C, Sherratt S, Rodriguez-Galan A, Behrens R, Riley EM. Sustained immune complex-mediated reduction in cd16 expression after vaccination regulates nk cell function. Front Immunol. 2016; 7:384. doi:10.3389/fimmu.2016.00384.

62. Kinlough CL, Poland PA, Bruns JB, Harkleroad KL, Hughey RP. Muc1 membrane trafficking is modulated by multiple interactions. J Biol Chem. 2004; 279:53071-53077. doi:10.1074/jbc.M409360200. 


\section{Supplementary data}

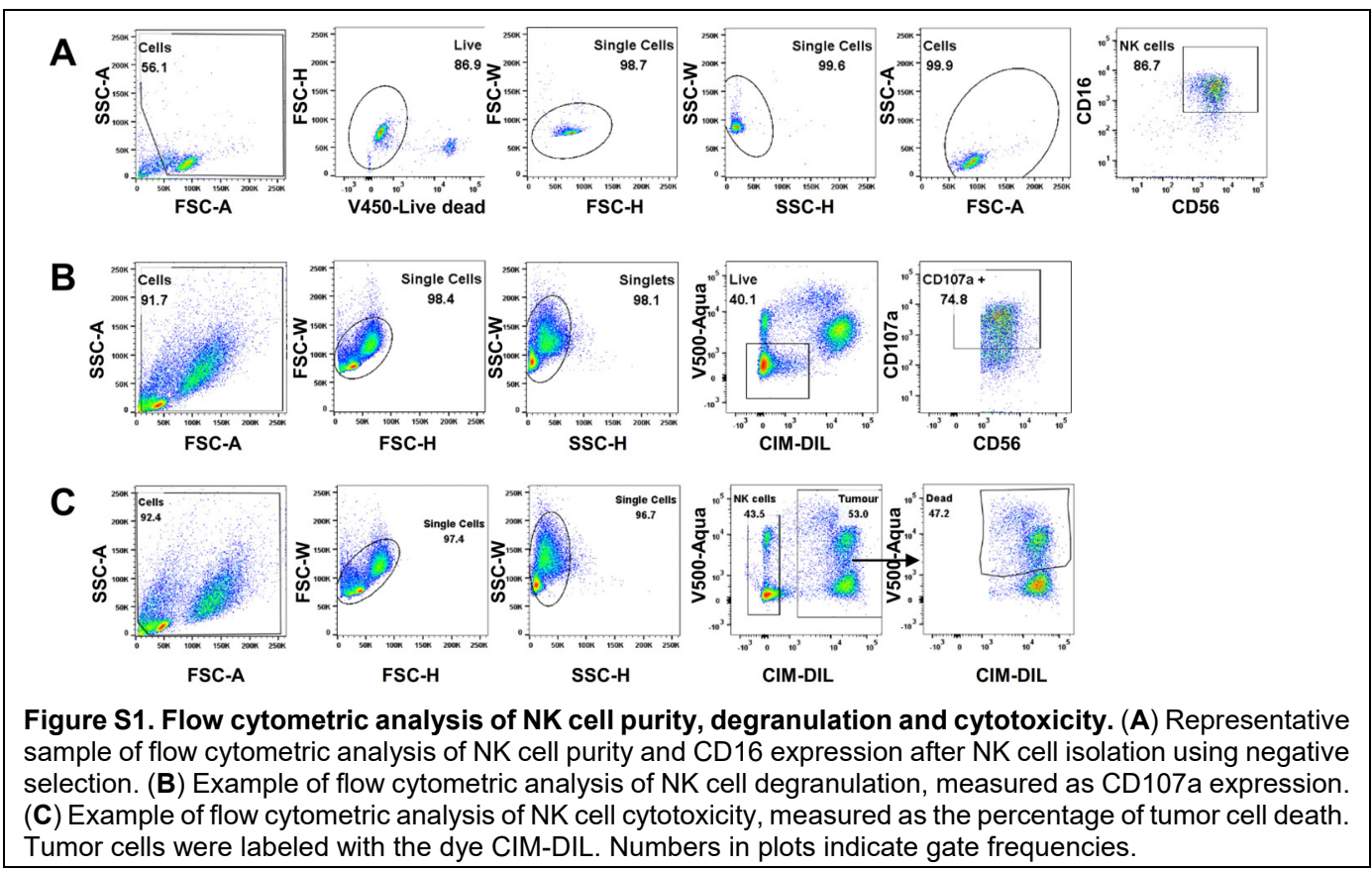




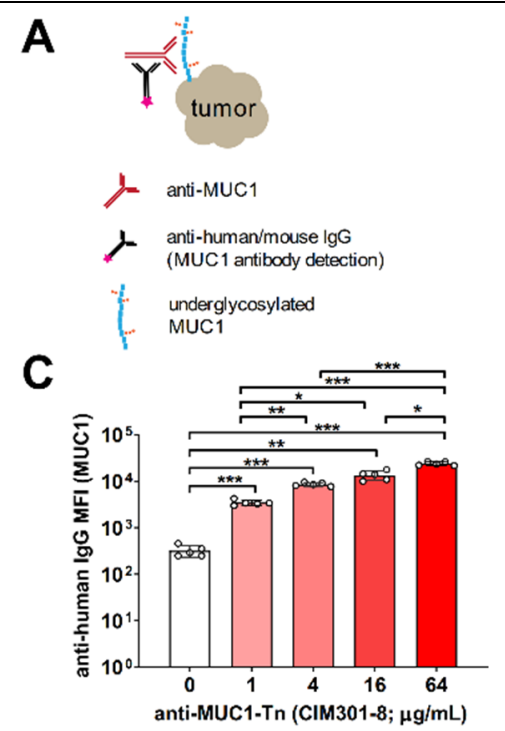

B

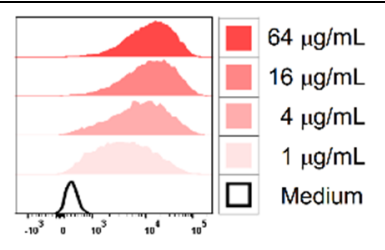

goat anti-human $\lg G$ AF647 (MUC1)

D

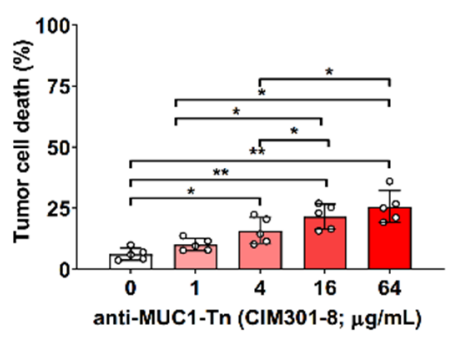

Figure S2. Higher concentrations of humanized anti-MUC1 antibodies induce Jurkat cell death. (A) Schematic overview of the analysis of the effects of increasing concentration of defucosylated CIM301-8 antiMUC1-Tn/STn antibodies on Jurkat tumor cells. (B) Overlay histogram showing binding of anti-MUC1 antibodies (CIM301-8; defucosylated Fc-tail) to Jurkat cells, detected using a labeled anti-human IgG antibody. One representative sample is shown. (C) Quantification of anti-MUC1 CIM301-8 binding to Jurkat cells as described in B, expressed as median fluorescence index. (D) Quantification of anti-MUC1-induced cell death of Jurkat cells after incubation with increasing concentrations of CIM301-8 antibodies (without NK cells). Differences between groups were calculated using one-way ANOVA with Tukey's multiple comparisons test. Not significant (n.s.); $p<0.05\left(^{*}\right) ; p<0.01\left(^{* *}\right) ; p<0.0001\left(^{* * *}\right)$. 


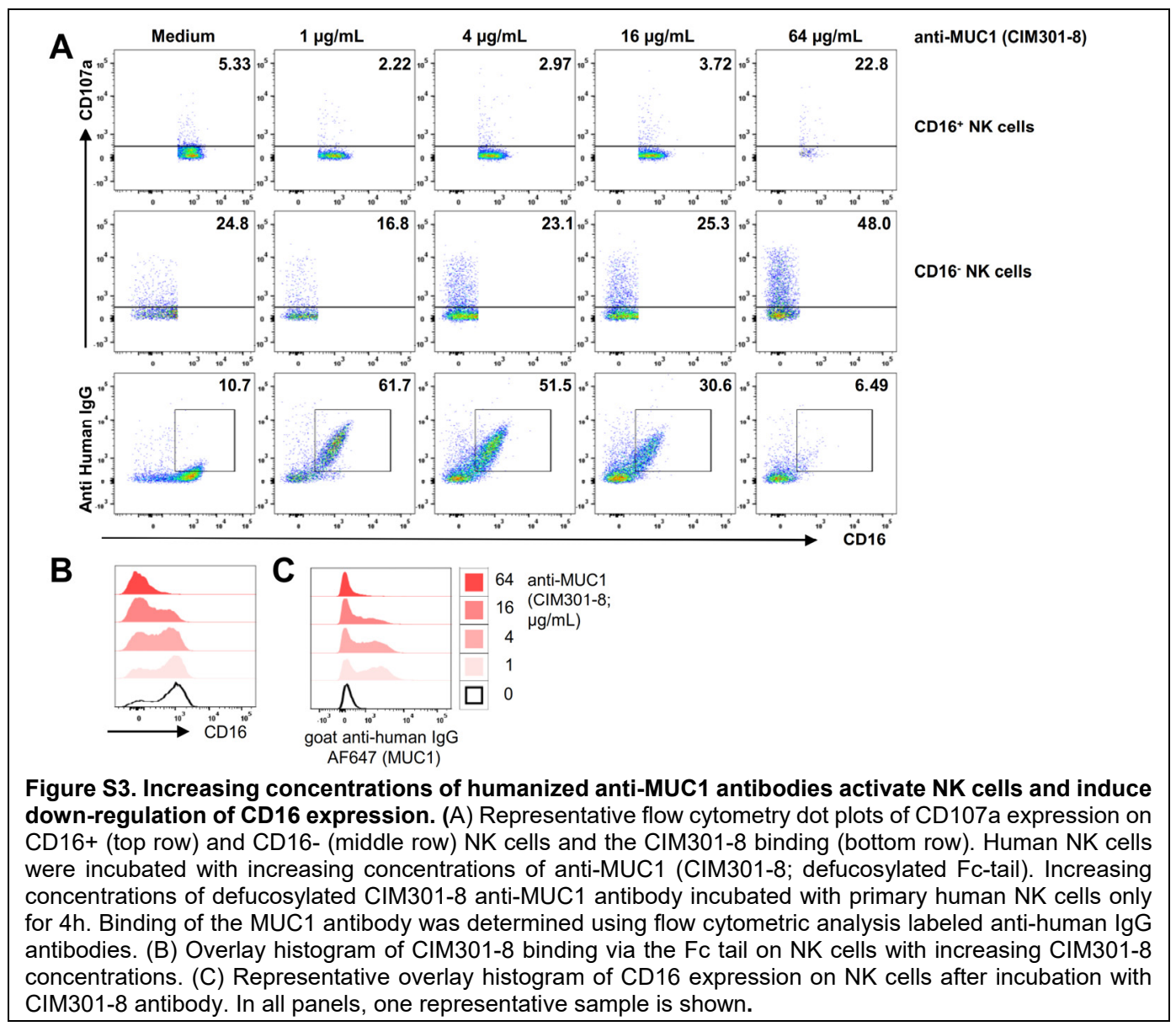




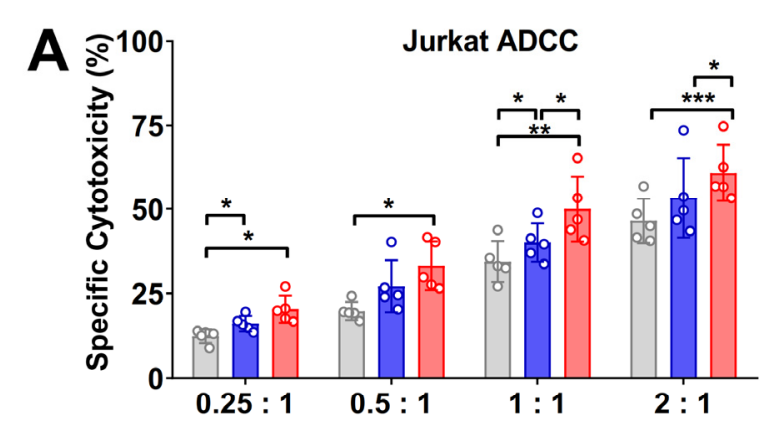

T-47D ADCC

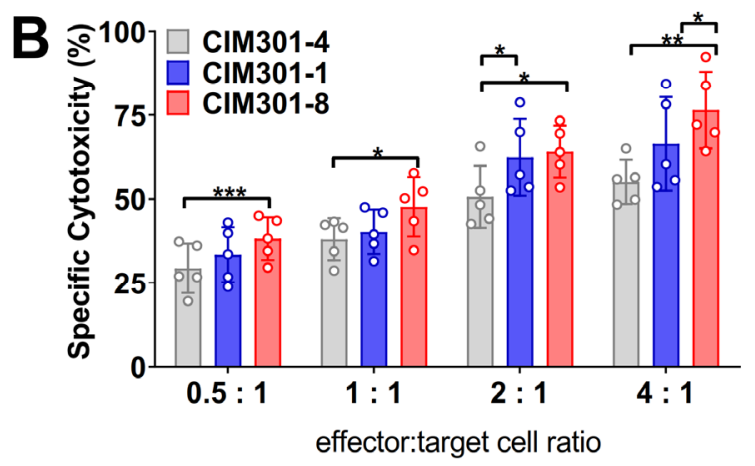

Figure S4. Humanized anti-MUC1 antibody enhances NK cell cytotoxicity via ADCC. (A) Quantification of antibody-dependent cellular cytotoxicity against Jurkat cells at different E:T ratios and in the presence of the indicated antibody at $1 \mu \mathrm{g} / \mathrm{mL}$ after $4 \mathrm{~h}$ of co-culture. Bars indicate mean $\pm \mathrm{SD}$, dots are 5 individual NK cell donors from independent experiments. (B) As A, with T-47D as tumor cells. Statistical analysis using two-way ANOVA plus Tukey's multiple comparisons test. Not significant (n.s.); $p<0.05\left(^{*}\right) ; p<0.01\left(^{(*}\right) ; p<0.001\left(^{(\star \star}\right)$. 

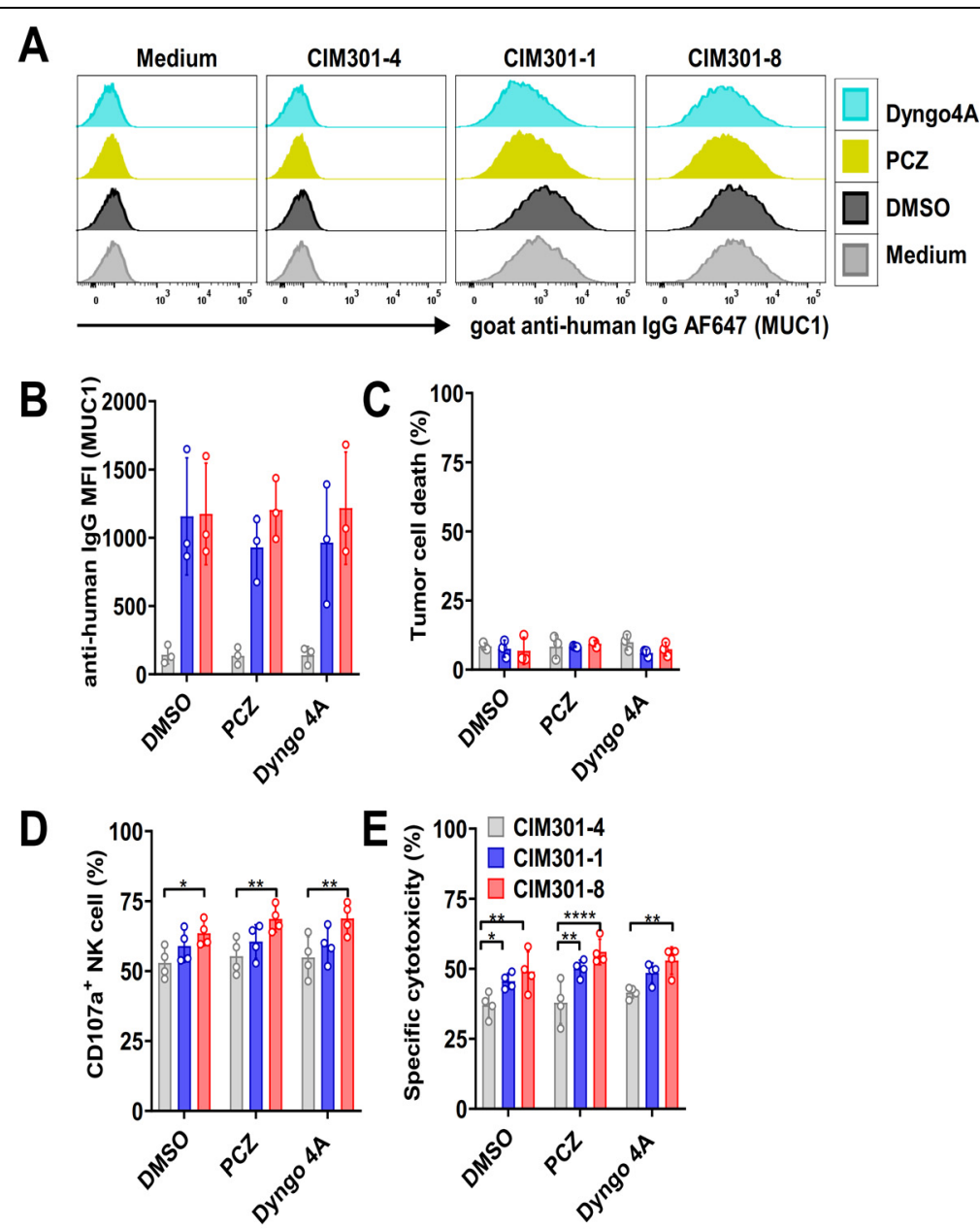

Figure S5. The endocytosis inhibitors PCZ and Dyngo4A neither upregulate the antigens on the tumor surface nor enhance the ADCC of Jurkat cells. (A) Overlay histograms of flow cytometric analysis of MUC1 epitope expression on Jurkat tumor cells in the presence of endocytosis inhibitors using regular (CIM301-1) or defucosylated (CIM301-8) anti-MUC1 antibodies or an irrelevant control antibody (CIM301-4). Anti-human IgG antibodies were used to detect antibody binding on tumor cells. Endocytosis inhibitors were dissolved in DMSO, here used as a negative control. One representative sample is shown. (B) Flow cytometric quantification of MFI of MUC1 expression levels on Jurkat cells after treatment with endocytosis inhibitors as described in A. Differences between control antibody (CIM301-4; grey bars) and anti-MUC1 antibodies (regular CIM301-1 in blue and defucosylated CIM301-8 in red) were all statistically significant with $p<0.05$ or smaller. (C) Viability of Jurkat tumor cells after incubation with anti-MUC1 antibodies with or without endocytosis inhibitors. Pooled data from 3 independent experiments performed at different timepoints. (D) Fraction of CD107a+ degranulation human NK cells in co-cultures with Jurkat tumor cells at an effector/target ratio of 1:1 in the presence of anti-MUC1 antibodies and endocytosis inhibitors. NK cells and tumor cells were incubated for $4 \mathrm{~h}$, with endocytosis inhibitors (5 $\mu \mathrm{M} \mathrm{PCZ,} 30 \mu \mathrm{M}$ Dyngo4A and $0.1 \%(\mathrm{v} / \mathrm{v}) \mathrm{DMSO}$ ) added during the last hour. (E) Antibody-dependent NK-cell mediated cytotoxicity against Jurkat tumor cells. Experimental setup as in $D$. Panels $D$ and $E$ show pooled data from 4 independent experiments with different donors, performed at different time points. Differences between groups were determined using two-way ANOVA with Tukey's multiple comparisons test. Not significant (n.s.); $p<0.05\left(^{*}\right) ; p<0.01\left(^{* *}\right) ; p<0.0001\left(^{* * * *}\right)$. 



\section{Chapter 3}

Rosuvastatin enhances VSV-G lentiviral transduction of Natural Killer cells via upregulation of the Low-Density Lipoprotein receptor

Gong Y, Klein Wolterink RGJ, Janssen I, Groot AJ, Bos GMJ, Germeraad WTV. Mol Ther Methods Clin Dev. 2020; 17:634-646. doi:10.1016/j.omtm.2020.03.017. 


\section{Abstract}

Adoptive Natural Killer (NK) cell therapy is attaining promising clinical outcome in recent years, but improvements are needed. Genetic modification of NK cells with a tumor antigen-specific receptor on their surface coupled to intracellular signaling domains may lead to enhanced cytotoxicity against malignant cells. One of the most common approaches is by lentivirus-mediated transduction. However, NK cells are difficult to transduce and various methods have been attempted with different success rates. As the Low-Density Lipoprotein-Receptor (LDLR) is the receptor of vesicular stomatitis virus (VSV) and is expressed only at low levels on NK cells, we tested the potential of 5 statins and 5 non-statin compounds to increase the LDLR expression, thereby facilitating viral transduction. We found that the transduction efficiency of VSV$G$ pseudotyped lentivirus is augmented by statins that induced higher LDLR expression. In both NK-92 cells and primary NK cells the transduction efficiency increased after treatment with statins. Furthermore, statins have been reported to suppress NK cell cytotoxicity, however we showed that this can be completely reversed by adding geranylgeranyl-pyrophosphate (GGPP). Among the statins tested, we found that the combination of rosuvastatin with GGPP most potently improved viral transduction without affecting the cytotoxic. 


\section{Graphical Abstract}

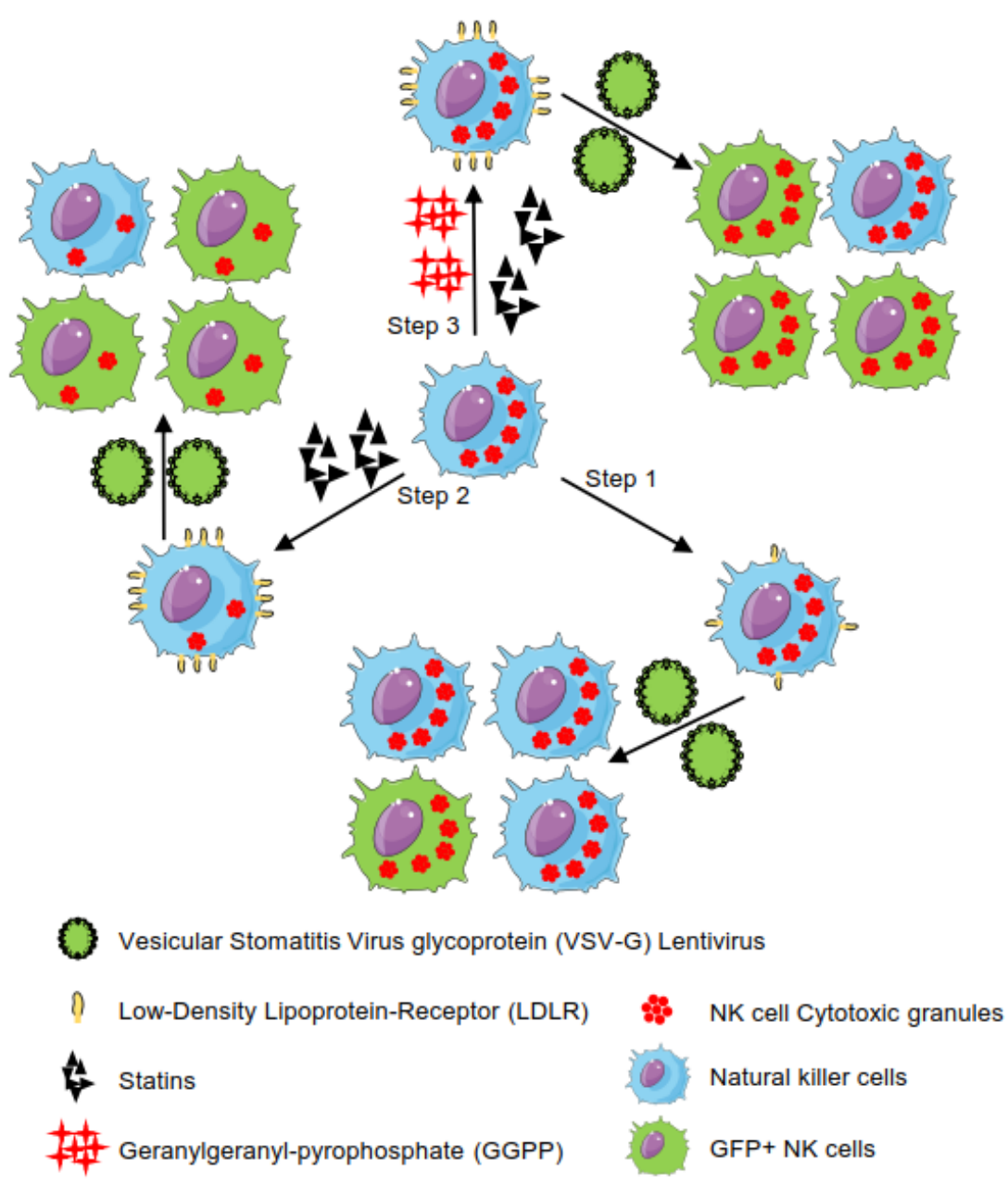

Natural Killer (NK) cells are known to be difficult to transduce and various methods have been attempted with different success rates. Gong and colleagues found that the combination of rosuvastatin with GGPP most potently improved VSV-G lentivirus transduction via upregulating cell surface LDLR without affecting the cytotoxic properties of the NK cells. 


\section{Introduction}

Cancer immunotherapy with its higher specificity and less side effects compared to traditional anti-cancer therapies has become an important tumor therapeutic strategy [1]. The main purpose is to break tolerance and revitalize the body's immune system that has become insensitive for advanced malignancies. The most prominent advantage of immunotherapy is the potential ability to eradicate distant metastases leading to a possible cure in a percentage of patients. A sophisticated form of immunotherapy is cellular therapy that includes dendritic cell vaccination, adoptive therapy of tumor infiltrate lymphocytes (TIL), tumor specific T cell receptor T cells (TCR-T), Natural Killer (NK) cells, as well as chimeric antigen receptor (CAR)-T and CAR-NK.

NK cells are innate immune cells having a surveillance function to eradicate virally infected cells or malignant cells. NK cells have several cytotoxic components leading to destruction of target cells. Perforin and granzyme incorporate into granules in the cytoplasm once NK cells are educated. After NK cells have recognized and bound target cells, the granules merge with the cell membrane and they secrete their content to mediate the killing process of the target cell by inducing apoptosis along the caspase pathways. One of the main mechanisms of NK cells to become active and cytotoxic is the concept of "missing-self". NK cells can recognize cells that are missing major histocompatibility complex (MHC) class I and thereby become activated. Moreover, NK cells can directly recognize tumor cells independent of $\mathrm{MHC}$ presentation like T cells [2]. The other mechanisms by which NK cells kill, include the death receptors FasL and TRAIL, also leading to apoptosis, but are different in terms of timing [3].

Adoptive transfer of mature alloreactive NK cells was shown to be effective in the treatment of patients with acute myeloid leukemia (AML) and to prevent relapse [4]. In contrast to the percentage of T cells, NK cells comprise a relatively small population and are not persistent as long as T cells in vivo.

With the development of genetic modification methods and promising clinical outcome of gene engineered T cells, NK cells could be great effector cells once armed with a specific antigen ligand or antibody. Building on the first clinical successes and subsequent application of the CD19 CAR-T for B cell hematological malignancies, there are increasing CAR-T or, more recent, CAR-NK clinical trials in progress to treat cancer patients $[5,6]$.

Generating CAR-T or CAR-NK cell consists of a genetic modification of cells resulting in surface expression of the antigen binding part of an antibody coupled to intracellular $\mathrm{T}$ cell or NK cell signaling molecules. This structure endows the T cell or NK cell to directly recognize the native tumor antigen[7], resulting in stronger cell activation and enhanced cytotoxicity. Despite the sensational clinical results with CAR-T cells, including their long in vivo persistence, it comes with the potential of various side effects; especially a cytokine release storm and neurotoxicity may cause dramatic outcomes and even death [8]. In this concept, NK cells with their short life span and high killing capacity could form an alternative and effective cell therapy [4]. Furthermore, combining a bestof-both-worlds concept, a CAR-NK cell can be generated.

Genetic modification to generate CAR-NK cells is aimed to improve their killing ability and tumor antigen targeting capacity. However, high efficiency of transfection or transduction of NK cells remains a big challenge. Retrovirus or lentivirus are the transfer methods of choice to obtain permanent integration of the transgene with high transduction efficiencies. Numerous reagents have been used to enhance viral 
transduction. Protamine sulfate or polymers (dextran or polybrene) can eliminate the electronic charge on the cell membranes [9]. Cyclosporine A [10] and rapamycin relieve distinct lentiviral restriction blocks in hematopoietic stem and progenitor cells [11]. Tolga et al. [12] reported that inhibition of intracellular antiviral defense mechanisms augments lentiviral transduction of human NK cells. Vectofusin-1 [13] and Prostaglandin E2 [14] and Dextran [15] have been reported to enhance lentiviral vector transduction of human hematopoietic stem cells (HPSC), T Lymphocytes [16] , and primary NK cells [15], respectively, without further mechanistic description.

Vesicular stomatitis virus surface glycoprotein (VSG-G) can be used as an envelope protein on the lentiviral particles [17] and the low density lipids (LDL) receptor and its family members serve as the cellular VSV receptors in human primary lymphocytes [18]. Upregulation of the LDL Receptor on lymphocytes may improve the VSV-G lentiviral transduction [19]. Interestingly, various groups have shown that the expression levels of LDLR in human B and T lymphocytes can be increased using antibodies, cytokines and estrogen receptor modulators $[19,20]$. Clinicians used statins as anti-hyperlipidemia drugs because they will upregulate the LDL receptor on endothelial cells thereby increasing lipid removal from the blood. However, in NK cells the impact of LDLR expression and its modulators has not been investigated. Therefore, we investigated which compounds influence the LDLR expression levels on NK cells and how LDLR expression levels improve lentiviral transduction efficiency of NK cells while NK cells ultimately maintain their cytotoxic capacity. 


\section{Materials and Methods}

\subsection{Cell lines and culture}

NK-92 cells (CRL-2407, ATCC, Manassas, USA) were cultured in alpha Minimum Essential medium (Thermo Scientific, Waltham, USA) without ribonucleosides and deoxyribonucleosides, supplemented with $2 \mathrm{mM}$ L-glutamine, $2.2 \mathrm{~g}$ sodium bicarbonate (Sigma Aldrich, Munich, Germany), $0.2 \mathrm{mM}$ inositol (Sigma Aldrich), $0.1 \mathrm{mM}$ 2mercaptoethanol (Sigma Aldrich), $0.02 \mathrm{mM}$ folic acid (Sigma Aldrich), $100 \mathrm{U} / \mathrm{ml}$ recombinant IL-2 (Proleukin, Novartis, Basel, Switzerland), 12.5\% horse serum (ATCC), $12.5 \%$ fetal bovine serum (FCS, Greiner bio-one, Frickenhausen, Germany) and $1 \%$ penicillin/ streptomycin (Thermo Scientific). K562 (ATCC CCL-243) cells were cultured in IMDM supplemented with 10\% FCS (Greiner bio-one) and 1\% penicillin/streptomycin (Thermo Scientific). 293FT cells (R700-07, Thermo Scientific) were cultured in DMEM/high glucose medium supplement with $10 \%$ FCS (Greiner bio-one), $0.1 \mathrm{mM}$ MEM Non-Essential Amino Acids (NEAA, Thermo Scientific), $2 \mathrm{mM}$ L-glutamine (Thermo Scientific), $1 \mathrm{mM}$ MEM Sodium Pyruvate (Thermo Scientific). The Jurkat cell line (ACC 282, DSMZ, Braunschweig, Germany) was cultured in RPMI-1640 Medium (Thermo Scientific) with $10 \%$ FCS and $1 \%$ penicillin/streptomycin.

\subsection{Human NK cell isolation and activation}

Primary human NK cells were isolated from anonymous buffy coats (Sanquin, Maastricht, The Netherlands). The use of buffy coats, being a by-product of a required Medical Ethical Review Committee (METC) procedure, does not need ethical approval in The Netherlands under the Dutch Code for Proper Secondary Use of Human Tissue. NK cells were subsequently isolated by negative selection with an NK cell isolation kit (130-092-657, Miltenyi Bbiotec, Bergisch Gladbach, Germany) using MACS beads as previously described [21]. For short-term activation, NK cells were cultured in RPMI1640 medium (Gibco) supplemented with 10\% fetal calf serum (Greiner Bio-One), 100 $\mathrm{U} / \mathrm{mL}$ penicillin-streptomycin (Gibco). NK cells were activated overnight with $1000 \mathrm{IU} / \mathrm{mL}$ recombinant human IL-2. All cells were cultured with $5 \% \mathrm{CO}_{2}$ at $37^{\circ} \mathrm{C}$ in a humidified cell culture Sanyo MCO-20AIC incubator (Sanyo Electric Co, Osaka, Japan).

\subsection{Co-culture of NK cells with statins and non-statin compounds}

NK-92 cells were seeded at $0.1 \times 10^{6}$ cells $/ \mathrm{mL}$ in round-bottom 96 well plates (3799, Corning Life Sciences B.V., Amsterdam, The Netherlands). Atorvastatin (10493, Cayman Chemical), fluvastatin (10010337, Cayman Chemical), pravastatin (10010342, Cayman Chemical), rosuvastatin (12029, Cayman Chemical) and simvastatin (10010344, Cayman Chemical) were dissolved in DMSO (Sigma Aldrich). Statins were used at final concentration of $20 \mu \mathrm{M}, 5 \mu \mathrm{M}$ and $0.5 \mu \mathrm{M}$. DMSO was diluted in the same volume and served as solvent control. Interleukin-2 (IL-2), IL-21 (Thermo Scientific), vitamin C (Sigma Aldrich), dextran (Sigma Aldrich), prostaglandin E2 (PGE2) (Sigma Aldrich), protamine sulfate (Sigma Aldrich) and vectofusin-1 (Miltenyi-biotec) were dissolved in distilled water (Gibco). DMSO was added $0.8 \mu \mathrm{L}, 0.2 \mu \mathrm{L}$ and $0.02 \mu \mathrm{L}$ respectively same volume as statin group did in 96 -well. IL-21 was added at $20 \mathrm{ng} / \mathrm{mL}$, 
$5 \mathrm{ng} / \mathrm{mL}$ and $0.5 \mathrm{ng} / \mathrm{mL}$. Vitamin C was used at concentrations of $500 \mu \mathrm{g} / \mathrm{mL}, 50 \mu \mathrm{g} / \mathrm{mL}$ and $5 \mu \mathrm{g} / \mathrm{mL}$ as described previously [22]. Dextran was used at $80 \mu \mathrm{g} / \mathrm{mL}, 8 \mu \mathrm{g} / \mathrm{mL}$ and $0.8 \mu \mathrm{g} / \mathrm{mL}$ [15]. PGE2 was used at $100 \mu \mathrm{M}, 10 \mu \mathrm{M}$ and $1 \mu \mathrm{M}$ [14] Vectofusin-1 was used at $50 \mu \mathrm{g} / \mathrm{mL}, 5 \mu \mathrm{g} / \mathrm{mL}$ and $0.5 \mu \mathrm{g} / \mathrm{mL}[16,23]$. Geranylgeranyl pyrophosphate ammonium salt (GGPP) was purchased from Sigma-Aldrich and was added at $10 \mu \mathrm{M}$ in co-culture assays [24].

\subsection{Vectors and lentivirus production and NK cells transduction}

All the virus procedures were under the surveillance by the Center for Research Innovation, Support and Policy (CRISP) of Maastricht University Medical Center (Genetic Modification License number: GGO-00-177). pCDH-EF1-copGFP-T2A-Puro was a gift from Kazuhiro Oka (Addgene plasmid \# 72263, Watertown, MA, USA). pRSVRev (Addgene \# 12253), pMDLg/ pRRE (Addgene \# 12251) and pMD2.G (Addgene \# 12259) were gifts from Dr. Didier Trono [25]. Plasmids were expanded in Stbl3 ${ }^{\mathrm{TM}} E$. coli (Thermo Scientific) and prepared by followed the instruction of NucleoBond Xtra Midi Kit (Macherey-Nagel GmbH, Düren, Germany). All these four plasmids were mixed with the ratio 5:2:2:1 in $40 \mu \mathrm{g}$ and were transfected in one $150 \mathrm{~mm}$ cell culture dish of 293FT cells in the presence of $80 \mu \mathrm{g}$ polyethylenimine (PEI) (Polysciences, Warrington, PA, USA). After 48 hours, every 24 hours and continually for 5 days virus supernatant was collected and filtered through a $0.45 \mu \mathrm{m}$ syringe filter (Merck Millipore, Burlington, USA). Pooled virus supernatant was concentrated using Lenti-X Concentrator (Takara, SaintGermainen-Laye, France) according to the manufacturer's protocol. Viral titers were determined using Jurkat cells by performing $2 x$ times' serial dilutions and detection of GFP expression using flow cytometry $48 \mathrm{~h}$ post-transduction. VSV-G Lentiviruses were added to cells at a Multiplicity Of Infection (MOI) 10 in the presence of $10 \mu \mathrm{g} / \mathrm{mL}$ protamine sulfate after 36 hours of statin co-incubation.

\subsection{Flow cytometry and antibodies staining}

For flow cytometric analysis, cells were stained with Live/Dead ${ }^{\circledR}$ Fixable Aqua Dead Cell Stain Kit (Thermo Scientific) in PBS on ice for 30 minutes. Then, cells were stained using the following antibodies recognizing human antigens: CD3 (Clone OKT3, BD Biosciences, San Jose), CD56 (Clone REA196, Miltenyi Biotec), LDLR (Clone C7, BD Biosciences), CD95L (Clone NOK-1, BD Biosciences), CD107a (Clone H4A3, Miltenyi Biotec), Granzyme B (Clone REA226, Miltenyi Biotec) and IFN- $\gamma$ (REA600, Miltenyi Biotec). Antibodies mixture in FACS staining buffer (PBS+1\% FCS) were added to NK cells and incubated at $4{ }^{\circ} \mathrm{C}$ for 30 minutes. Fluorescence was read on a BD FACS Canto II flow cytometer. Data were analysed with FlowJo 10.1 (TreeStar, Ashland, USA) software.

\subsection{Cytotoxicity assay}

The NK cell killing ability against tumor cells was determined in a 4 hours flow cytometry-based assay. Tumor cells were pre-labeled with Cell Tracker ${ }^{\mathrm{TM}}$ Deep Red Dye according to the manufacturer's protocol (Thermo Scientific) and were cultured overnight. Tumor cells were harvested and seeded at 20,000 cells per well in round- 
bottom 96 well plates. NK cells were seeded in duplicates with different effector to target ratios (E/T ratio) and cultured in RPMI1640 medium for 4 hours. After 4 hours, dead Deep Red-labeled tumor cells were measured with Live/Dead ${ }^{\circledR}$ Fixable Aqua Dead Cell Stain Kit (Thermo Scientific) by flow cytometry. Specific cytotoxicity was calculated as previous described [21].

\subsection{Statistical analysis}

All statistical tests used in this study were completed with GraphPad Prism 8 software (Graphpad Software Inc, San Diego, USA). The specific statistical tests used for each comparison are specifically annotated in the figure legends, respectively. For performing multiple comparisons, we used two-way ANOVA and Bonferroni post-tests comparing to the medium control group. For cell viability, Mann-Whitney $U$ test was used to compare with the medium control group. ${ }^{*}$ indicates a $p$ value of $<0.05$, ${ }^{* *}$ indicated $p<0.01$, ${ }^{* * *}$ indicates $p<0.001$. Results were considered non-significantly (n.s.) different if $p \geq 0.05$. 


\section{Results}

\subsection{Statins enhance LDLR expression levels in the NK-92 cell line}

Given that LDLR expression levels in human B and T lymphocytes can be influenced using compounds compatible with in vitro culture, we first asked what drugs influence LDLR expression levels in human NK cells. For screening purposes, we made use of the human NK cell line NK-92. This cell line shares important features with primary NK cells: it recognizes viruses and tumor cells, has cytotoxic capabilities and produces characteristic NK cell cytokines [26]. Based on previous publications, we tested compounds that have been reported to enhance NK cell transduction (IL-21 [27] and dextran [15]), enhance lentiviral transduction in hematopoietic stem cells and T lymphocytes (vectofusin-1 [16], prostaglandin E2 [14]) and promote NK cell proliferation (asorbic acid) [22]. Furthermore, we tested statins (HMG-CoA reductase inhibitors) that are clinically used as lipid-lowering medication [28] and that have been reported to directly increase $L D L R$ mRNA in human mononuclear cells [29]. Here, we examined the effects of three lipophilic statins (atorvastatin, fluvastatin and simvastatin) and two hydrophilic statins (pravastatin and rosuvastatin).

Previously, Hillyard et al. [30] had demonstrated that $10 \mu \mathrm{M}$ statin is detrimental for the NK cell membrane raft, which is a key functional component for NK cell cytotoxicity. The same statins concentration was also shown by Poggi et al. [31], who reported that $10 \mu \mathrm{M}$ fluvastatin was able to decrease the activation markers on NK cells. Initially, we tested three different concentrations of statins: $0.5 \mu \mathrm{M}, 5 \mu \mathrm{M}$ and $20 \mu \mathrm{M}$. Higher doses of $50 \mu \mathrm{M}$ resulted in a $50 \%$ reduction in viability after 36 hours incubation and were therefore not used (data not shown). Culture of NK-92 cells with the various statins at these concentrations did not have a significant negative impact on cell viability (Figure 1A). Similarly, most non-statin compounds, except dextran, did not negatively impact NK-92 cell viability, even at the highest concentrations tested (Figure 1A).

Flow cytometric analysis showed that all statins tested increased the LDLR protein expression at least 3-fold, while none of the other compounds significantly upregulated LDLR expression levels (Figures 1B and 1C). LDLR expression levels were not strictly dose-dependent: we observed that LDLR expression levels were generally highest following culture in $5 \mu \mathrm{M}$ of the respective statins (Figure 1C), without a negative impact on cell viability (Figure 1A). Therefore, we determined $5 \mu \mathrm{M}$ to be the optimal concentration to augment the expression of LDLR on NK-92 cells. Statins at a higher concentration inhibit the viability of NK cells, whereas at a low $0.5 \mu \mathrm{M}$ LDLR expression was not highly induced. The major impact of the statins is apparent within 12 hours (Figure S1A) and the induction of LDLR expression on NK-92 cells following statin stimulation is time-dependent. Moreover, addition of statins also impacted on cell viability, as 48 hours after statin administration, the NK cells that normally grow in clumps loosened, resulting in a gradual decrease in overall viability (Figure S1B), while LDLR expression levels did not increase further (Figure S1A). Therefore, we determined $5 \mu \mathrm{M}$ to be the optimal concentration and 36 hours incubation for statins to upregulate LDLR expression in NK-92 cells, since LDLR expression was highest at this concentration with no negative impact on cell viability (Figure S1B and S1C). 
A

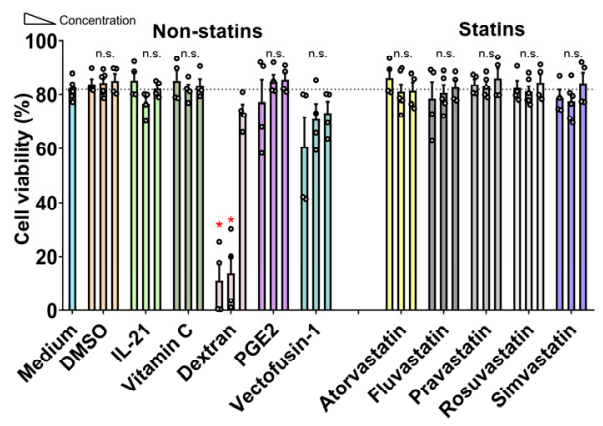

B
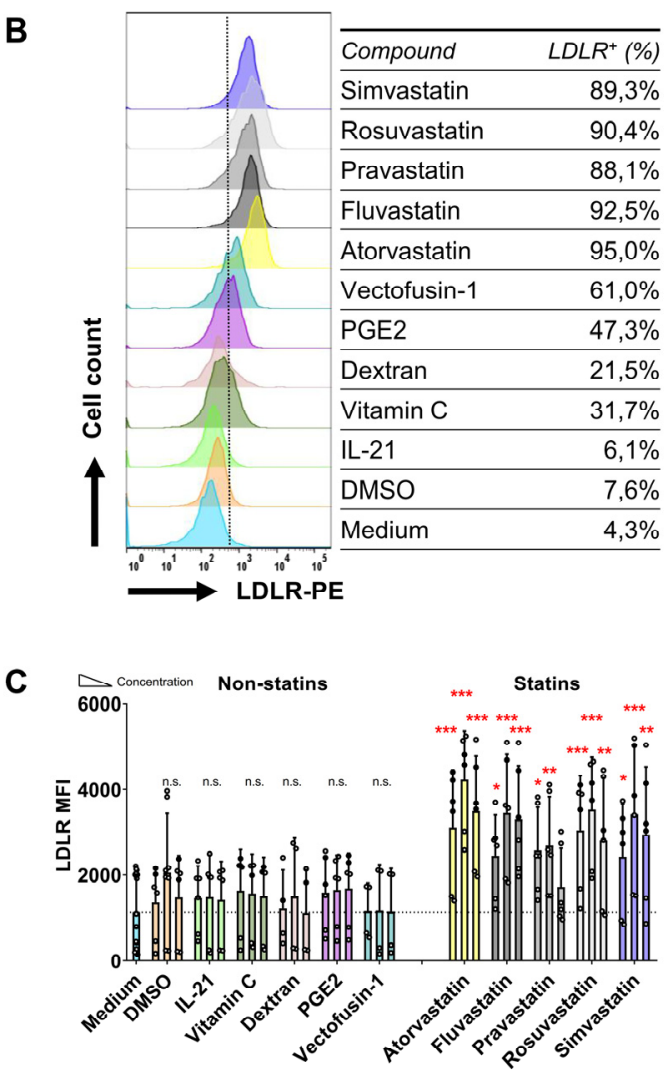

Figure 1. LDL-receptor up-regulated by statins on NK-92 cells.

(A) Viability of NK-92 cells after 36 hours of co-culture with different concentrations of compounds in MEM medium with $100 \mathrm{U} / \mathrm{mL}$ IL-2. Dead cells were stained by the fixable Aqua V500. NK-92 cells were seeded at $0.1 \times 10^{6} / \mathrm{mL}$ in 96 well plates, $200 \mu \mathrm{L}$ in each well. IL-21 was added at $20 \mathrm{ng} / \mathrm{mL}, 5 \mathrm{ng} / \mathrm{mL}$ and $0.5 \mathrm{ng} / \mathrm{mL}$. Vitamin $C$ was used at concentrations of $500 \mu \mathrm{g} / \mathrm{mL}, 50 \mu \mathrm{g} / \mathrm{mL}$ and $5 \mu \mathrm{g} / \mathrm{mL}$. Dextran was used at $80 \mu \mathrm{g} / \mathrm{mL}$, $8 \mu \mathrm{g} / \mathrm{mL}$ and $0.8 \mu \mathrm{g} / \mathrm{mL}$. PGE2 was used at $100 \mu \mathrm{M}, 10 \mu \mathrm{M}$ and $1 \mu \mathrm{M}$. Vectofusin-1 was used at $50 \mu \mathrm{g} / \mathrm{mL}, 5$ $\mu \mathrm{g} / \mathrm{mL}$ and $0.5 \mu \mathrm{g} / \mathrm{mL}$. All the statins were used at $20 \mu \mathrm{M}, 5 \mu \mathrm{M}$ and $0.5 \mu \mathrm{M}$. Concentration height is from left to right, indicated with the triangle. DMSO was used as solvent for the statins and was taken along as negative control (B) LDLR expression level of NK-92 cells after 36 hours co-culture with the compounds is expressed as median fluorescence intensity (MFI). Overlay histogram of LDLR expression on NK-92 cells under every middle concentration of all compounds are displayed. The table indicates the fraction of LDLR-positive cells. (C) LDLR levels were up-regulated by statins, but not by non-statin compounds. Data are shown as mean \pm 
SD; Pooled data from 4 independent experiments performed at different times. Data analysis was performed by a two-way ANOVA and Bonferroni post-tests in comparison to medium. For cell viability, Mann-Whitney $U$ test was used to compare with medium group.

\subsection{Statins enhance the viral transduction efficiency of NK-92 cells}

Next, we determined whether the statin-induced increase of cell surface LDLR expression levels in NK-92 cells lead to enhanced VSV-G lentiviral transduction efficiency. Thus, we treated cultured NK-92 cells with statins for 36 hours, followed by lentiviral transduction with a GFP-encoding vector and analysis after 48 hours (Figure 2A and 2B). We used flow cytometric analysis of the fraction of GFP-positive cells and their mean fluorescence index (MFI) to determine the lentiviral transduction efficiency. As reported by other groups $[7,27]$, lentiviral transduction of NK-92 cells has a mild negative impact on cell viability (Figure 2C). However, in combination with lentiviral transduction, most statins had unexpectedly a profound impact on cell viability. Notably, this effect was less pronounced in the cultures treated with the hydrophilic statins' pravastatin and rosuvastatin compared with the lipophilic statins (Figure 2C). Importantly, atorvastatin, rosuvastatin and simvastatin significantly increased the fraction of GFP-expressing cells and the GFP expression levels with 1.5- to 2.5-fold, indicating improved transduction efficiency (Figures 2D and 2E). In the non-statins group, only dextran promoted transduction efficiency at the high expense of cell viability. Interestingly, higher doses of statins did not further enhance GFP expression levels after lentiviral transduction (Figure 2E), while the observed negative effects of statins on cell viability of lentivirus transduced NK-92 cells were dose-dependent (Figure 2C). Pooled analysis of the effect of LDLR expression levels on transduction efficiency showed that higher LDLR expression analysis correlated with higher transduction efficiency (Pearson correlation co-efficient $r=0.6750, p<0.0001$ ) (Figure $2 F$ and $2 \mathbf{G}$ ). In addition, we observed that higher virus titers resulted in higher GFP expression levels, but also lead to more cell death (Figure S2). Therefore, we determined a MOI of 10 to be the optimal virus concentration for the transduction of NK-92 cells. Statins enhanced lentiviral transduction efficiency of NK-92 cells around 2-fold when treated with atorvastatin, rosuvastatin or simvastatin. While lentiviral transduction in the presence of statins has a negative impact on cell viability, this effect is dose-dependent, and a $5 \mu \mathrm{M}$ dose of statins can be used without major negative effects on cell viability. 
62 | Chapter 3

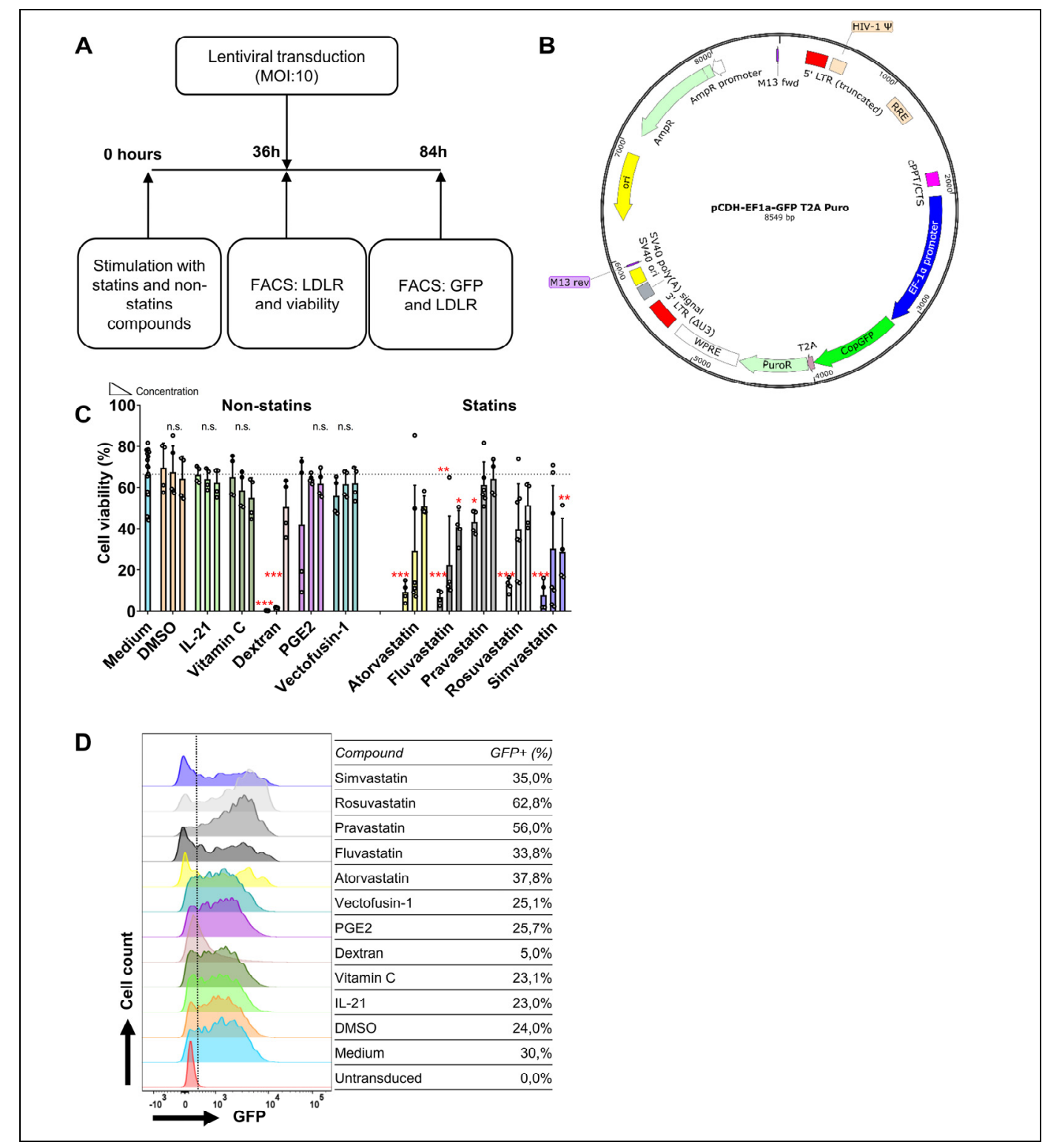



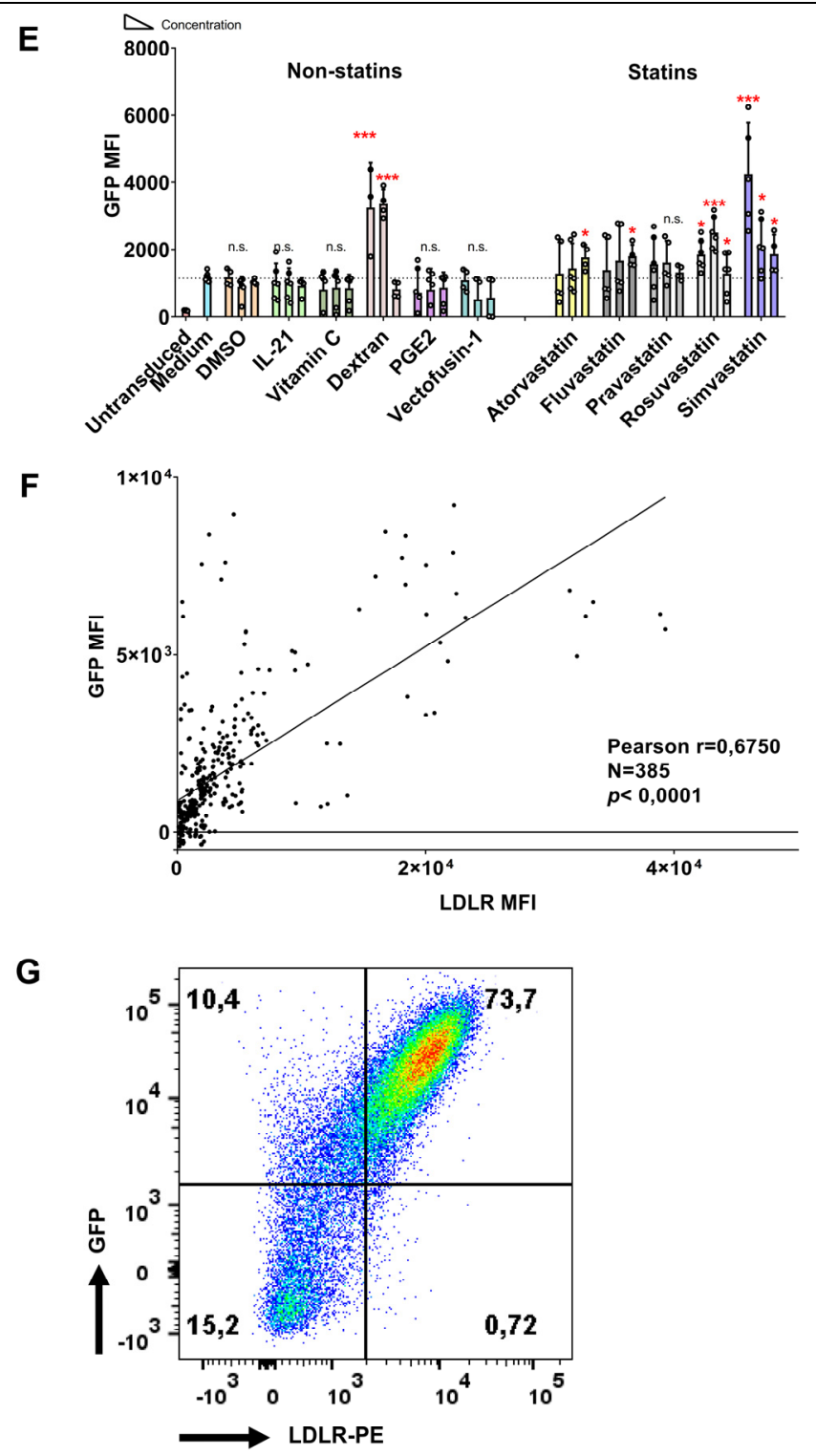

Figure 2. NK-92 cell transduction efficiency is enhanced by statins.

(A) Flow chart of procedures using NK-92 cells for transduction with or without compounds. (B) Map of 3rd generation lentiviral vector pCDH-EF1a-GFP-puro. (C) Viability of NK-92 cells 48 hours after viral transduction, following culture in the presence of the different compounds at the indicated concentrations. After co-culturing for 36 hours, medium was exchanged in each well. Dead cells were detected by fixable Aqua V500. (D) Flow cytometric overlay histograms of GFP expression in NK-92 cells cultured with the middle concentration of each compound. VSV-G lentivirus was added at the MOI of $10: 1$ in the presence of $10 \mu \mathrm{g} / \mathrm{mL}$ protamine sulfate for transduction. (E) NK cell transduction efficiency was determined by GFP expression in living NK-92 cells. (F) Pearson correlation analysis between LDLR MFI before transduction and 48hours after transduction of NK-92 cells. (G) One representative example of LDLR co-expression with GFP on NK-92 48 hours after viral transduction was showed. Data shown as mean $\pm S D$. Pooled data from $N=4$ independent experiments performed at different times. 


\subsection{Statin-induced reduction in NK cell cytotoxicity is reversed by GGPP}

NK cell immunotherapy builds on the intrinsic cytotoxic capacity of the cells. For NK cells, it has previously been demonstrated that statins can inhibit their cytotoxic capacity[30,32]. Indeed, using the NK-92 cells, we observed almost complete inhibition of cytotoxicity against the K562 chronic myelogenous leukemia (CML) cell line 48 hours after lentiviral transduction in the presence of atorvastatin, fluvastatin or simvastatin, while cytotoxicity was slight decreased in the presence of rosuvastatin (Figure 3A). However, pravastatin did not alter the cytotoxic capacity of NK-92 cells on K562 cells. Interestingly, geranylgeranyl-pyrophosphate (GGPP), a key molecule in biosynthesis downstream pathways of HMG-CoA reductase [24], could completely reverse the suppression statin-induced NK cell cytotoxicity (Figure 3B), while maintaining the statininduced increase in lentiviral transduction (Figure 3C). We also tested if the cytokine IL2 , that is commonly used to active NK cells and NK cell lines, could reverse the negative effects of statins on NK cell cytotoxicity, as IL-2 was previously shown to be sufficient to overcome the negative impact of statins on cytotoxicity [33]. However, in NK-92 cells, a high dose of IL-2 (1000 U/mL) alone was not sufficient to restore their cytotoxic capacity (Figure S3).

To unravel what NK cell cytotoxic pathway was impacted by statins, we analyzed expression of the degranulation marker CD107a, granzyme B, apoptosis-inducing FAS ligand and IFN-y secretion (Figure S4) Indeed, we observed downregulation of CD107a and granzyme B levels in statin-treated NK-92 cells compared to control cells (Figure S4A and S4B). Importantly, these effects were reversed by GGPP, while GGPP did not influence any of the other analyzed cytotoxicity pathways. Thus, statins can importantly reduce NK-92 cell cytotoxicity, but GGPP can fully reverse this effect completely (Figure 3C). 

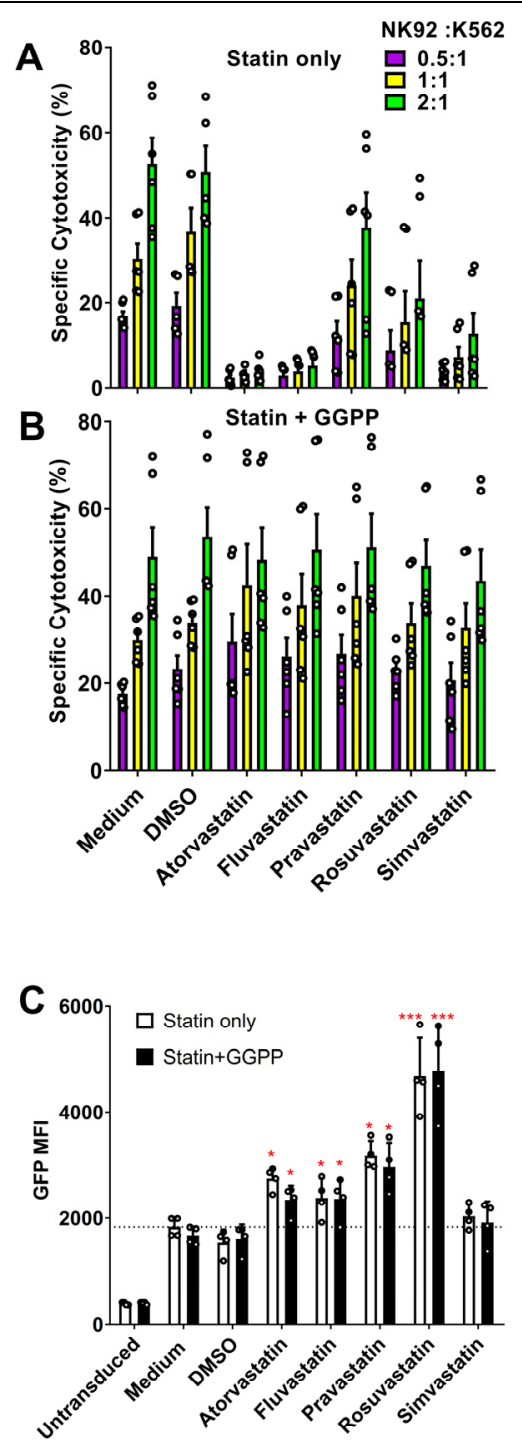

Figure 3. Statins inhibited NK-92 cell cytotoxicity, which could reverse by GGPP without influencing GFP expression.

(A) NK-92 cell cytotoxicity was suppressed by $5 \mu \mathrm{M}$ lipophilic statins after 36 hours incubation. However, NK92 cells' cytotoxicity was not changed by pravastatin and not $100 \%$ restrained by rosuvastatin. NK-92 cells were counted after 36 hours exposure to statins and mixed in specified ratios of effector cells to target cells (E/T ratio) in 96 well-plates. K562 target cells were seeded at 20,000 cells per well. The cytotoxicity assay was performed during 4 hours. Cytotoxicity was quantified as percentage dead target cells using flow cytometry. (B) The inhibition of statins on NK-92 cell cytotoxicity could be reversed by $10 \mu \mathrm{M}$ GGPP. Data shown is one representative experiment out of 3 independent assays. (C) The statin induced enhancement of NK-92 cells transduction efficiency was not altered by GGPP. NK-92 cells were incubated with statins in the presence of GGPP or not during 36 hours. Then, culture medium was refreshed and VSV-G virus at MOI of 10:1 was added. Data was showed as mean $\pm \mathrm{SD}$ and derived from $\mathrm{N}=4$ independent experiments performed at different times. Data analysis was performed by pair signed-rank test between statins only group and statins plus GGPP group. 


\subsection{Statins upregulate LDLR expression and enhance the viral transduction efficiency of primary human NK cells}

While lymphoma-derived NK-92 cells have been used for clinical applications[34], human primary NK cells are a heterogeneous population of cells that in general shows better cytotoxic capacities [35]. We checked freshly isolated human primary NK cells, and found that the CD56 dim population expressed more LDLR than the CD56 bright population (Figure S5A and S5B). Therefore, we next tested if statins can also be used to enhance lentiviral transduction via LDLR upregulation in primary human NK cells. First, we confirmed that statins $(5 \mu \mathrm{M})$ and the non-statin compounds did not negatively affect NK cell viability (Figure 4A). The only exception was dextran, which as previously shown had a marked negative impact on NK-92 viability (Figure 1A). In the statin-treated groups, we observed a trend of LDLR upregulation, which was only significantly upregulated 2-fold in the rosuvastatin-treated NK cells (Figures 4B and 4C). Further analysis of LDLR expression showed that most likely only a subset of primary NK cells responds to statin-treatment (Figure 4C).

We subsequently transduced primary NK cells with the GFP-expression lentiviral vector that we used before to transduce NK-92 cells (Figure 2A). In contrast to NK-92 cells, primary NK cells can be transduced in the presence of statins or non-statins without significant negative effects on cell viability. However, we did not observe significant differences in viability after treatment with statins (Figure 5A). Meanwhile, we observed a 2- to 3-fold increase in GFP expression levels in statin-treated NK cells compared to the non-statin control group (Figures 5B and 5C). 48 hours after transduction, genomic DNA was extracted from the VSV-G transduced primary NK cells and PCR was used to confirm integration of transgene (Figure S6). Indeed, LDLR expression levels positively correlated with GFP expression levels after lentiviral transduction (Figure 5D and 5E; Pearson correlation co-efficient $r=0.6159, p<0.0001$ ), demonstrating that rosuvastatin that induce LDLR expression can be used to enhance lentiviral transduction. 

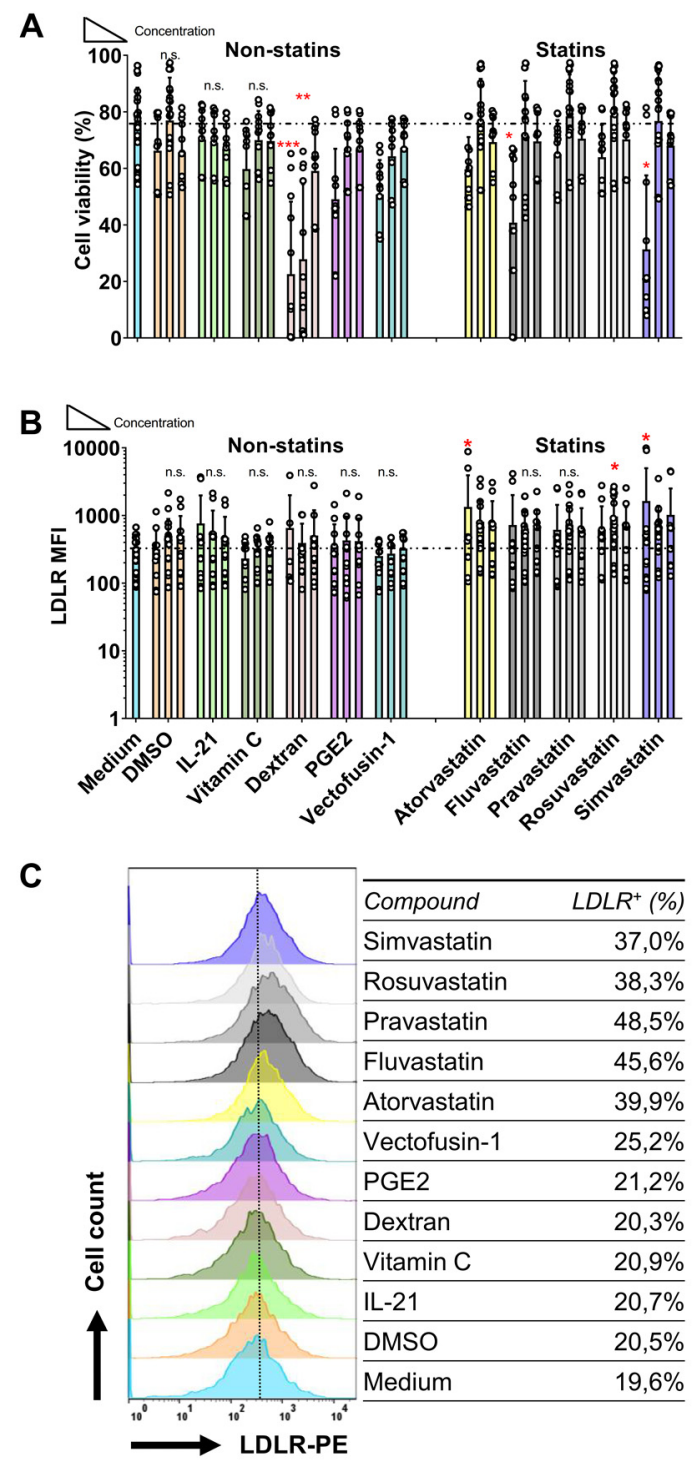

Figure 4. LDL-receptor up-regulation by statins on human primary NK cells.

(A) Viability of NK cells after 36 hours stimulation with different compounds as previous described. Human primary NK cells were co-cultured with compounds for 36 hours at a density of $0.1 \times 10^{6} / \mathrm{mL}$. NK cells were pre-activated overnight in RPMI 1640 with $1000 \mathrm{U} / \mathrm{mL}$ IL-2. (B) The LDLR expression on primary NK cell was up-regulated by rosuvastatin, however, in the non-statin groups no significant difference was found when compared to medium. (C) Overlay histogram of LDLR expression on NK cells stimulated 36 hours with different compounds. Data are from $\mathrm{N}=6$ independent experiments performed at different times. Data analysis was performed by a two-way ANOVA and Bonferroni post-tests in comparison to medium. For cell viability, MannWhitney $\mathrm{U}$ test was used to compare with medium group. 


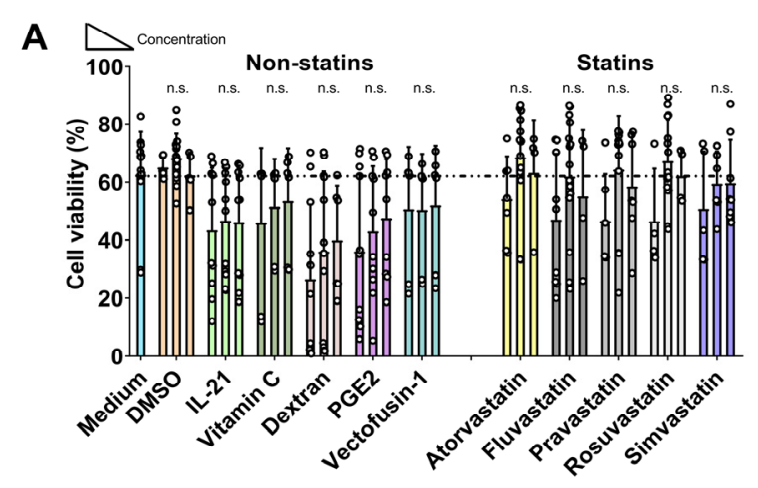

B

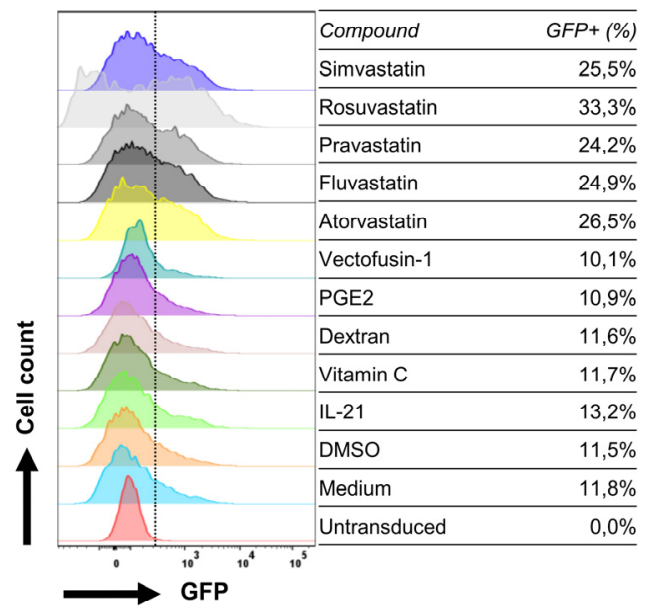

C

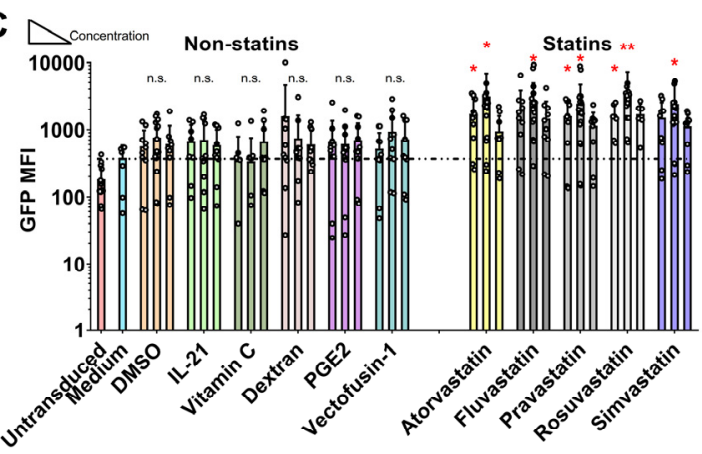

Figure 5 Lentiviral transduction of human primary NK cells.

(A) Viability of primary NK cells 48 hours after transduction with different compounds at the indicated concentrations. (B) Flow cytometric overlay histogram of GFP expression in NK cells. VSV-G lentivirus was added at the $\mathrm{MOI}$ of $10: 1$ in the presence of $10 \mu \mathrm{g} / \mathrm{mL}$ protamine sulfate. (C) NK cell transduction efficiency was determined by GFP expression in living NK cells. GFP expression was higher after culture with rosuvastatin when compared with DMSO and medium group. 


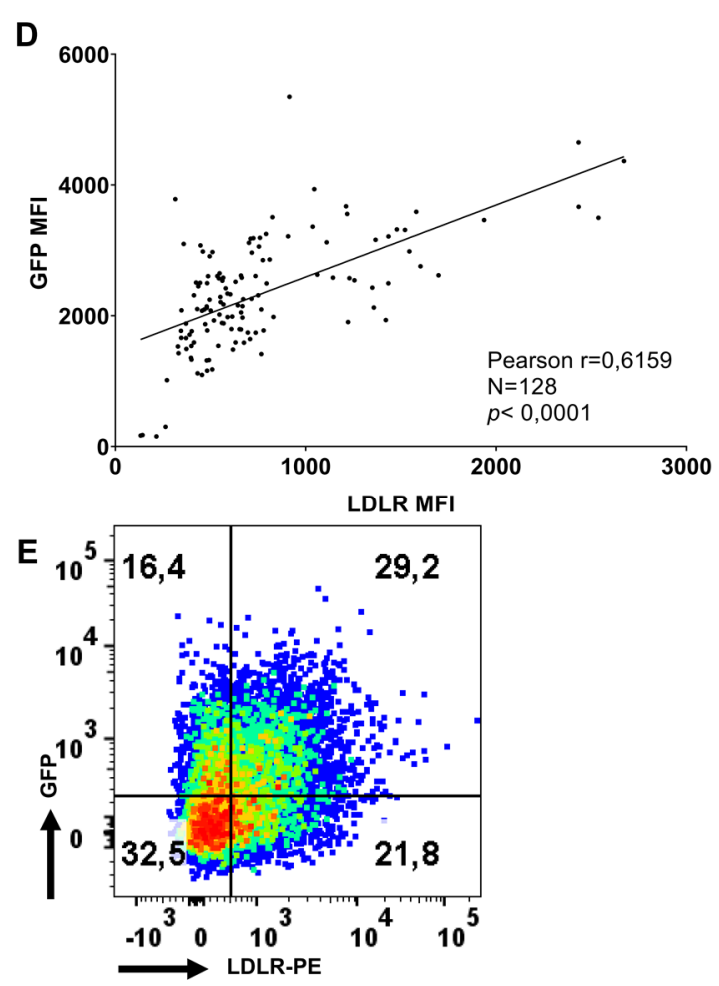

Figure 5. Lentiviral transduction of human primary NK cells.

(D) Pearson correlation analysis of LDLR MFI (before transduction) and GFP MFI (48 hours after transduction) on NK cells. (E) One representative LDLR co-expression with GFP on NK after 48 hours viral transduction. Data was showed as mean $\pm S D$ from $N=6$ independent experiments performed at different times. Data analysis was performed by a two-way ANOVA and Bonferroni post-tests in comparison to medium. For cell viability, Mann-Whitney $U$ test was used to compare with medium group.

\subsection{Rosuvastatin plus GGPP promotes lentiviral transduction through an increase of LDLR expression on the surface of primary NK cells}

Statins upregulated the LDLR expression (Figure 6A) without negative effects on cell viability (Figure 6B). As for NK-92 cells, higher LDLR expression levels enhanced VSV$G$ lentiviral transduction in the presence of GGPP (Figure 6C), without concerning the viability before or after transduction on primary NK cells (Figure 6B and 6D). Above all, among 5 kinds of statins, Rosuvastatin was the most potent compound to both upregulate the LDLR on NK cells and increase the transduction. The LDLR and GFP were co-expressed in NK-92 (Figure 6E), while primary NK cells have more GFP cells when the LDLR was higher expressed (Figure 6F). After 36 hours statins stimulation, the killing ability of primary NK cells in rosuvastatin was decreased. Fortunately, this suppressive effect was completely reversed by GGPP (Figure 7). Thus, a combination of rosuvastatin with GGPP in the culture medium augmented the transduction efficiency of primary NK cells. 

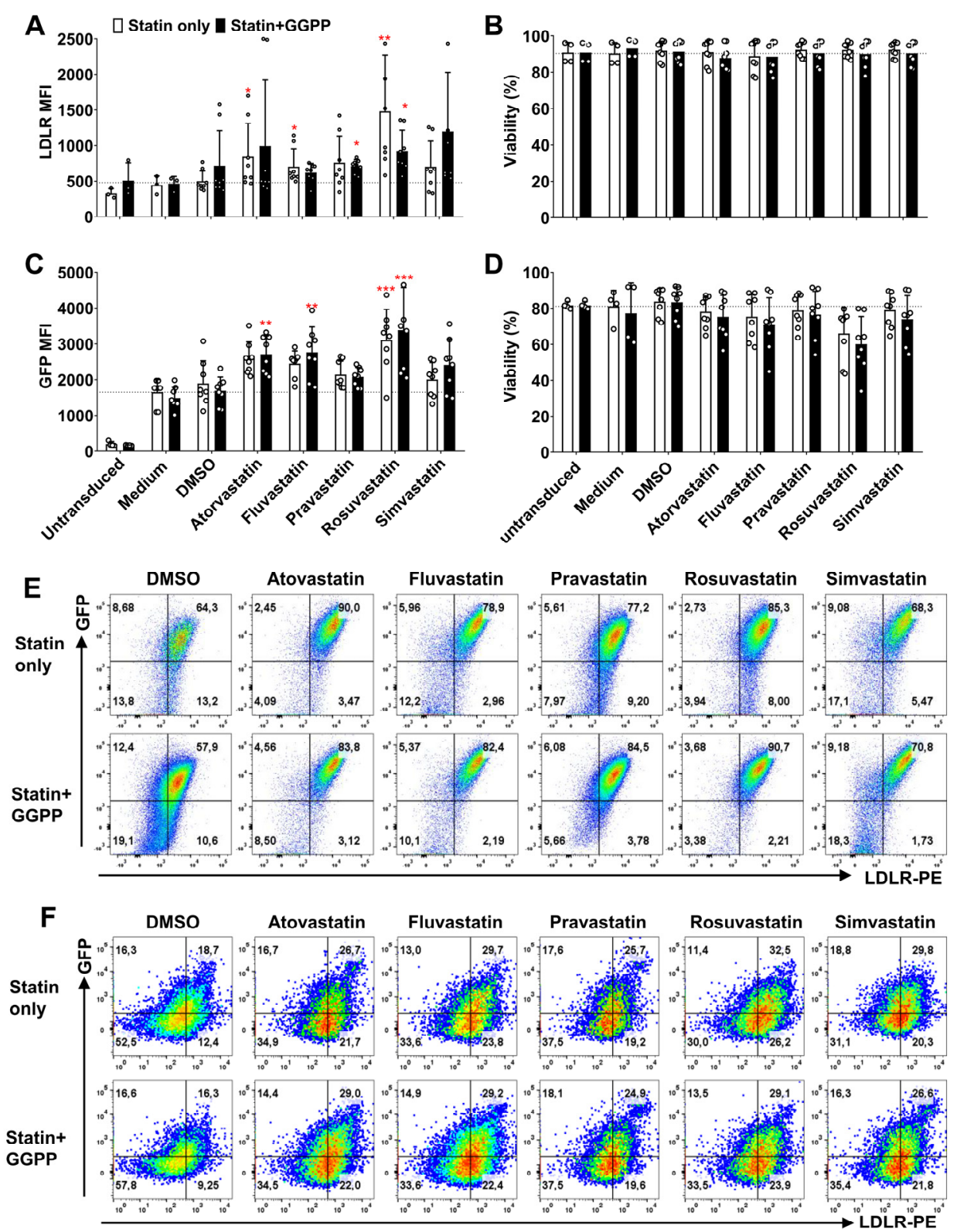

Figure 6. Statins with GGPP enhance the transduction efficiency of NK-92 and primary NK cells.

(A) LDL-receptor expression on primary NK cells after 36 hours stimulation with $5 \mu \mathrm{M}$ statins, with or without $10 \mu \mathrm{M}$ GGPP. Primary NK cells were co-cultured with $5 \mu \mathrm{M}$ statins in the presence or absence of GGPP at a density of $0.1 \times 10^{6} / \mathrm{mL}$ (B) Viability of primary NK cells after 36 hours cultured in the presence of $5 \mu \mathrm{M}$ statins with or without $10 \mu \mathrm{M}$ GGPP. (C) GFP expression in primary NK cells after lentiviral transduction. After statins incubation, the culture medium was refreshed and VSV-G virus added at MOI of 10:1. (D) Viability of primary NK cells after 48 hours transduction. Representative flow cytometry LDLR and GFP co-expression in NK-92 (E) and primary NK cells $(F)$ after 48 hours lentiviral transduction and culturing with statins with or without GGPP. Data is showed as mean $\pm S D$ from $N=4$ independent experiments performed at different times. Data analysis was performed by a two-way ANOVA and Bonferroni post-tests in comparison to medium. For cell viability, Mann-Whitney $U$ test was used to compare with medium group. 


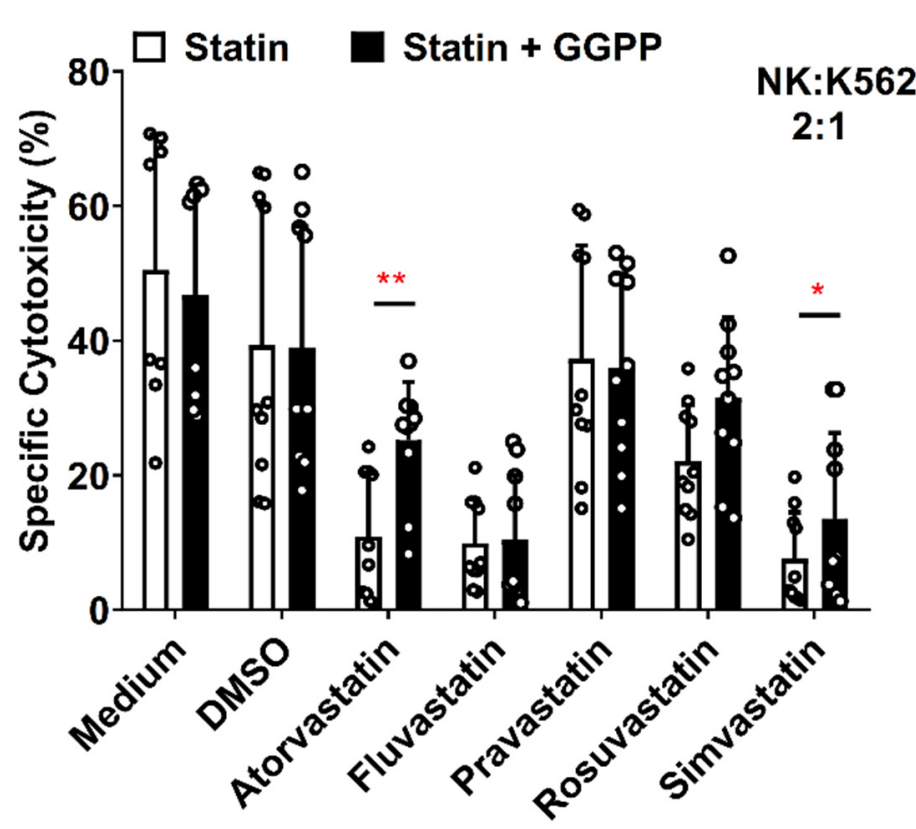

Figure 7. Cytotoxicity of primary NK cells is inhibited by statins, but restored by GGPP.

Human primary NK cell cytotoxicity was suppressed by lipophilic statins. NK cell cytotoxicity was not changed by pravastatin and restrained by rosuvastatin. NK cells were incubated with statins in presence of GGPP or not. NK cells were seeded at the density of $0.1 \times 10^{6} / \mathrm{mL}$ in 96 well plates with or without statins. After removing the supernatant, NK cells were added in a 2:1 E: T ratio: 40,000 cells NK cells vs 20,000 K562 cells per well. Cytotoxicity assay was performed for 4 hours. The inhibition of statins on primary NK cell cytotoxicity could only be completely reversed by addition of $10 \mu \mathrm{M}$ GGPP after rosuvastatin stimulation. Data was showed from 4 independent assays. Data analysis was performed by pair signed-rank test between statins only group and statins plus GGPP group. 


\section{Discussion}

Achieving high and effective transduction remains a big hurdle for the application of adoptive transfer of genetically modified NK cell therapies in the clinic [7]. Numerous methods have been explored to improve lentiviral transduction efficiency [36]. Here, we tested various statins and showed their enhancing effect on the transduction efficiency on NK cells by increasing the LDLR expression, receptor for VSV-G. The average increased ratio of the LDLR expression by statins both on NK-92 cells and primary human NK cells is approximately 1.5 - 3 times. The data in this study showed that, compared with the other statins, the lipophilic drug rosuvastatin most potently upregulated LDLR expression on NK cells, resulting in increased transduction efficiency. The addition of GGPP completely reversed the negative impact of statins on NK cell cytotoxicity.

The main biological function of statins is induction of LDLR expression on the cell surface of liver cells through inhibition of hepatic cholesterol synthesis [37]. Upregulation of LDLR results in increased clearance of serum cholesterol. Clinical data shows a significant difference in prevention of coronary artery disease between patients treated with lipophilic or hydrophilic statins [38],[39]. The biological function of statins was shown to be dose-dependent with rosuvastatin seemingly having the highest activity [40]. Here, we used statins to increase the LDLR expression to allow VSV-G pseudotyped viruses to enter the NK cell more efficiently. We observed the LDLR expression increasing is time course dependent of statins at $5 \mu \mathrm{M}$ (Figure S1), however LDLR is not dose dependent both in NK-92 (Figure 1B) and primary NK cells (Figure 4B). A similar study demonstrated that LDLR expression is not dose dependent [41]. We also found no clear dose-dependent effects of the statins at the different doses tested $(0.5 \mu \mathrm{M}, 5 \mu \mathrm{M}$ and 20 $\mu \mathrm{M})$. However, we found that high concentrations of statins (more than $20 \mu \mathrm{M}$ ) induced cell death, possibly due to negative effects on overall cell metabolism, thereby also inhibiting LDLR expression levels. Similarly, lower concentration of statins $(<0.5 \mu \mathrm{M})$ may not be enough to induce LDLR high expression. The inhibitory effects on proliferation and cytotoxicity by statins have been reported in several studies [24,32]. When compared with lipophilic statins (e.g. simvastatin and fluvastatin), hydrophilic statins (including pravastatin and rosuvastatin) showed milder or no suppression of proliferation and functional capacity on IL-2 stimulated NK cells (Figure 3) [42]. The viability of NK-92 cells in the medium control group before and after transduction decreased from $80 \%$ decrease to $70 \%$ (Figure 1A and 2C), while in primary NK cells, cell viability decreased from $75 \%$ to $65 \%$ (Figure 4A and $5 \mathrm{~A}$ ). Before transduction, were found no differences in cell viability in NK-92 cells or primary NK cells between the statintreated group and the non-statin group. However, after VSV-G lentivirus transduction, the $20 \mu \mathrm{M}$ of statins group showed negative effects on the viability of both NK-92 cells and primary NK cells. This effect is mainly due to the toxicity of VSV-G protein and protamine sulfate, with an additional detrimental effect of statins on NK cell viability. The inhibitory effects of statins on NK cell cytotoxicity could, among others, be due to the reduction of NK-target (tumor) cell adhesion, granule exocytosis (perforin and granzymes release) $[30,31]$. The release of cytotoxic lytic granules from NK cells is 
strongly dependent on phosphatidylinositol-specific phospholipase $\mathrm{C}_{\gamma}$ (PLCy) phosphorylation and intracellular free calcium [43]. Whether statins alter the abundance of intracellular $\mathrm{Ca}^{2+}$ in NK cells is debated: Raemer et al. [32] showed simvastatin had no influence on $\mathrm{Ca}^{2+}$ flux in human primary NK cells, while Poggi et al [31] demonstrated fluvastatin reduced intracellular free $\mathrm{Ca}^{2+}$ concentration in human NK cell clones. This discrepancy might be due to the different statins used or NK cell source variation. Furthermore, statins have been reported to change the killing components of NK cells, such as CD95L (FasL) and interferon-y (IFN-y) [31]. In this study, we also investigated the CD107a degranulation, granzyme B, FasL expression and IFN-y secretion after coculture of NK cells with statins (Figure S4). As previous studies [30,31] already demonstrated, CD107a degranulation and FasL levels were reduced upon statin treatment. Interestingly, IFN- $y$ secretion was not altered. This could be due to cytokines and cell-cell contact with neighboring cells, which has been reported in earlier studies $[33,44]$. These results could imply that the statin-induced inhibition of NK killing capacity might be due to changes in the degranulation process.

As described in the results section, statins decreased NK cell cytotoxicity. However, this effect could be reversed completely by geranylgeranyl pyrophosphate (GGPP) [32]. GGPP is synthesized by HMG-CoA reductase and is independent from the cholesterol metabolism. GGPP or mevalonate reversed the inhibitory effects of statins on HMGCoA reductase [24]. Crosbie et al showed that GGPP or mevalonate was able to alter the cell cycle and DNA synthesis of NK cells, thus abrogating the negative effects of statins on NK cell proliferation $[24,45]$.

In addition to statins, other approaches have also been tested to improve high NK cell transduction to create CAR-NK cells [46]. Polycations like polybrene, dextran, polyL-lysine and protamine sulfate are supposed to eliminate the charges on cell membranes, thereby enhancing viral transduction efficiency $[15,47]$. In a direct comparison of polybrene, protamine sulfate and dextran sulfate [15], it was shown that dextran-treated NK cells show the highest transduction efficiency at $38 \%$ GFP positive cells, whereas no GFP expression was detected in cells treated with either polybrene or protamine sulfate. In contrast to the present study, their research showed that $8 \mu \mathrm{g} / \mathrm{mL}$ dextran had no influence on the viability and killing capacity of NK cells, while we observed that this concentration of dextran was detrimental for NK cell viability (below 20\%; Figure 1A). This difference might be due to the use of freshly isolated NK cells, while Nanbakhsh et al used expanded NK cells [15]. The second approach to increase higher NK cell transduction efficiency is by cytokine or mitogen stimulation. Previous studies demonstrated that LDLR upregulation, in conjunction with enhanced proliferation and cytotoxicity, can also be achieved by stimulation with IL-2 [48]. Soluble IL-2 plus IL-12 stimulation enhances VSV-G lentiviral transduction, which could be further enhanced by $1 \mu \mathrm{g} / \mathrm{mL}$ PHA [27]. Culturing primary human NK cells on K562 feeder cells with expressing membrane-bound IL-21 and 4-1BBL also augmented gene transduction by $50 \%[49,50]$. Thirdly, suppression of intracellular antiviral defense mechanisms is also described to increase lentiviral transduction of NK cells. BX795, which is an inhibitor of the TBK1/IKKE complex that controls antiviral responses, is able to boost lentiviral gene transduction efficiency by 3.8 fold [51]. Next, higher transduction efficiency may be achieved with other pseudotypes of viruses, like alpha-retroviral vectors [52] or baboon 
envelope pseudotyped lentivirus[50]. Finally, methods have also been developed to obtain genetically engineered NK cells from HSC [53] or induced pluripotent stem cells [54].

Statins also have anti-inflammatory and immunomodulatory properties in clinical treatment [55]. Given that statins facilitate the entry of lentiviruses into cells, this could render patients more susceptible to virus infection. Even though some small studies indicate that statins indeed promoted virus infection or activity (e.g. in herpes zoster [56] and respiratory viral infections [57]), other studies indicate that statins restrict virus activity (e.g. in HIV [58] and Ebola infection [59]), while a third group shows no effect $[57,60]$. Most retrospective studies have intrinsic methodological limitations and too few relevant randomized controlled trials have been performed on relation between statins and viral infectious incidence [60]. Altogether, at this time there is no body of evidence that allows us to draw definitive conclusions on the effects of clinical statin use on virus infections.

In the current paper, we describe a novel strategy to improve the transduction on human NK cells by increasing the expression of the LDLR. NK cells expressing higher LDLR levels on the surface could get easier transduced with VSV-G lentivirus by helping the VSV lentivirus entry into the NK cell (Figure 2F and 5D). Now the structural composition of how VSV-G recognizes the LDLR on the cell surface has been elucidated [61], we can imagine that specific overexpression of the cysteine-rich domains (CR2 or CR3) of the LDLR on NK cells could enhance the transduction efficiency. The limitation of the approach is that human primary NK cells express low levels of LDLR. However, other VSV-G receptors may be present on NK cell surface, like the leucine-rich repeatcontaining G protein-coupled receptor 4 (Lgr4) [62], HSP90B1 [63] and LDL-receptor other family members [18].

For future therapeutic applications, rosuvastatin plus GGPP currently is the most potent combination that increases VSV-G lentivirus transduction efficiency without a reduction of NK cell cytotoxicity. This finding is important for the both scientists and clinicians, as it facilitates the transduction of NK cells that are known to be hard to transduce, but hold important promise for cancer adoptive cell therapy. 


\section{Acknowledgements}

Y.G. thanks the China Scholarship Council (CSC) (No. 201707720056) for fellowship support. R.G.J.K.W. is supported by a Kootstra Talent Fellowship (Maastricht University) and a Marie Skłodowska-Curie Individual fellowship (799810-TOPNIN; European Union). The research was supported by Cancer Research Foundation Limburg (KOFL-2012-03) and GROW School of Maastricht University.

We thank the Maastricht University CRISP Virus production core facility. All the virus work in this study is under the GMO biosafety license: GGO-00-177 and GGO-17-006. 


\section{References}

1. Dunbar CE, High KA, Joung JK, Kohn DB, Ozawa K, Sadelain M. Gene therapy comes of age. Science. 2018; 359:eaan4672. doi:10.1126/science.aan4672.

2. Zhang C, Oberoi P, Oelsner S, Waldmann A, Lindner A, Tonn T, et al. Chimeric antigen receptorengineered nk-92 cells: An off-the-shelf cellular therapeutic for targeted elimination of cancer cells and induction of protective antitumor immunity. Front Immunol. 2017; 8:533. doi:10.3389/fimmu.2017.00533.

3. Screpanti V, Wallin RP, Grandien A, Ljunggren HG. Impact of fasl-induced apoptosis in the elimination of tumor cells by nk cells. Mol Immunol. 2005; 42:495-499. doi:10.1016/j.molimm.2004.07.033.

4. Miller JS, Soignier Y, Panoskaltsis-Mortari A, McNearney SA, Yun GH, Fautsch SK, et al. Successful adoptive transfer and in vivo expansion of human haploidentical nk cells in patients with cancer. Blood. 2005; 105:3051-3057. doi:10.1182/blood-2004-07-2974.

5. Liu E, Marin D, Banerjee P, Macapinlac HA, Thompson P, Basar R, et al. Use of car-transduced natural killer cells in cd19-positive lymphoid tumors. N Engl J Med. 2020; 382:545-553. doi:10.1056/NEJMoa1910607.

6. Suen WC-W, Lee WY-W, Leung K-T, Pan X-H, Li G. Natural killer cell-based cancer immunotherapy: A review on 10 years completed clinical trials. Cancer Investigation. 2018; 36:431-457. doi:10.1080/07357907.2018.1515315.

7. Carlsten M, Childs RW. Genetic manipulation of nk cells for cancer immunotherapy: Techniques and clinical implications. Front Immunol. 2015; 6:266. doi:10.3389/fimmu.2015.00266.

8. Nguyen DN, Roth TL, Li PJ, Chen PA, Apathy R, Mamedov MR, et al. Polymer-stabilized cas9 nanoparticles and modified repair templates increase genome editing efficiency. Nat Biotechnol. 2020; 38:44-49. doi:10.1038/s41587-019-0325-6.

9. Yang YW, Hsieh YC. Protamine sulfate enhances the transduction efficiency of recombinant adenoassociated virus-mediated gene delivery. Pharm Res. 2001; 18:922-927

10. Petrillo C, Cesana D, Piras F, Bartolaccini S, Naldini L, Montini E, et al. Cyclosporin a and rapamycin relieve distinct lentiviral restriction blocks in hematopoietic stem and progenitor cells. Mol Ther. 2015; 23:352-362. doi:https://doi.org/10.1038/mt.2014.193.

11. Wang CX, Sather BD, Wang X, Adair J, Khan I, Singh S, et al. Rapamycin relieves lentiviral vector transduction resistance in human and mouse hematopoietic stem cells. Blood. 2014; 124:913-923. doi:10.1182/blood-2013-12-546218.

12. Sutlu T, Nyström S, Gilljam M, Stellan B, Applequist SE, Alici E. Inhibition of intracellular antiviral defense mechanisms augments lentiviral transduction of human natural killer cells: Implications for gene therapy. Hum Gene Ther. 2012; 23:1090-1100. doi:10.1089/hum.2012.080.

13. Radek C, Bernadin O, Drechsel K, Cordes N, Pfeifer R, Sträßer P, et al. Vectofusin-1 improves transduction of primary human cells with diverse retroviral and lentiviral pseudotypes, enabling robust, automated closed-system manufacturing. Hum Gene Ther. 2019; 30:1477-1493. doi:10.1089/hum.2019.157.

14. Heffner GC, Bonner M, Christiansen L, Pierciey FJ, Campbell D, Smurnyy Y, et al. Prostaglandin e2 increases lentiviral vector transduction efficiency of adult human hematopoietic stem and progenitor cells. Mol Ther. 2018; 26:320-328. doi:10.1016/j.ymthe.2017.09.025.

15. Nanbakhsh A, Best B, Riese M, Rao S, Wang L, Medin J, et al. Dextran enhances the lentiviral transduction efficiency of murine and human primary nk cells. J Vis Exp. 2018; 131:55063. doi: $10.3791 / 55063$

16. Piovan C, Marin V, Scavullo C, Corna S, Giuliani E, Bossi S, et al. Vectofusin-1 promotes rd114-trpseudotyped lentiviral vector transduction of human hspcs and t lymphocytes. Mol Ther Methods Clin Dev. 2017; 5:22-30. doi:https://doi.org/10.1016/j.omtm.2017.02.003.

17. Burns JC, Friedmann T, Driever W, Burrascano M, Yee JK. Vesicular stomatitis virus g glycoprotein pseudotyped retroviral vectors: Concentration to very high titer and efficient gene transfer into mammalian and nonmammalian cells. Proc Natl Acad Sci U S A. 1993; 90:8033-8037. doi:10.1073/pnas.90.17.8033.

18. Finkelshtein D, Werman A, Novick D, Barak S, Rubinstein M. Ldl receptor and its family members serve as the cellular receptors for vesicular stomatitis virus. Proc Natl Acad Sci U S A. 2013; 110:7306-7311

19. Amirache F, Lévy C, Costa C, Mangeot P-E, Torbett BE, Wang CX, et al. Mystery solved: Vsv-g-lvs do not allow efficient gene transfer into unstimulated t cells, b cells, and hscs because they lack the Idl receptor. Blood. 2014; 123:1422-1424. doi:10.1182/blood-2013-11-540641. 
20. Cerrato F, Fernández-Suárez M, Alonso R, Alonso M, Vazquez C, Pastor O, et al. Clinically used selective oestrogen receptor modulators increase Idl receptor activity in primary human lymphocytes. British journal of pharmacology. 2015; 172:1379-1394

21. Mahaweni NM, Bos GMJ, Mitsiades CS, Tilanus MGJ, Wieten L. Daratumumab augments alloreactive natural killer cell cytotoxicity towards cd38+ multiple myeloma cell lines in a biochemical context mimicking tumour microenvironment conditions. Cancer Immunol Immunother. 2018; 67:861-872. doi:10.1007/s00262-018-2140-1.

22. Huijskens M, Walczak M, Sarkar S, Atrafi F, Gijsbers BLMG, Tilanus MGJ, et al. Ascorbic acid promotes proliferation of natural killer cell populations in culture systems applicable for natural killer cell therapy. Cytotherapy. 2015; 17:613-620. doi:10.1016/j.jcyt.2015.01.004.

23. Jamali A, Kapitza L, Schaser T, Johnston ICD, Buchholz CJ, Hartmann J. Highly efficient and selective car-gene transfer using cd4- and cd8-targeted lentiviral vectors. Mol Ther Methods Clin Dev. 2019; 13:371-379. doi:10.1016/j.omtm.2019.03.003.

24. Crosbie J, Magnussen M, Dornbier R, lannone A, Steele TA. Statins inhibit proliferation and cytotoxicity of a human leukemic natural killer cell line. Biomark Res. 2013; 1:33. doi:10.1186/20507771-1-33.

25. Dull T, Zufferey R, Kelly M, Mandel RJ, Nguyen M, Trono D, et al. A third-generation lentivirus vector with a conditional packaging system. J Virol. 1998; 72:8463-8471

26. Oelsner S, Friede ME, Zhang C, Wagner J, Badura S, Bader P, et al. Continuously expanding car nk-92 cells display selective cytotoxicity against b-cell leukemia and lymphoma. Cytotherapy. 2017; 19:235-249. doi:10.1016/j.jcyt.2016.10.009.

27. Micucci F, Zingoni A, Piccoli M, Frati L, Santoni A, Galandrini R. High-efficient lentiviral vectormediated gene transfer into primary human nk cells. Exp Hematol. 2006; 34:1344-1352. doi:https://doi.org/10.1016/j.exphem.2006.06.001.

28. Parihar SP, Guler R, Brombacher F. Statins: A viable candidate for host-directed therapy against infectious diseases. Nat Rev Immunol. 2019; 19:104-117. doi:10.1038/s41577-018-0094-3.

29. Pocathikorn A, Taylor RR, Mamotte CD. Atorvastatin increases expression of low-density lipoprotein receptor mrna in human circulating mononuclear cells. Clin Exp Pharmacol Physiol. 2010; 37:471476. doi:10.1111/j.1440-1681.2009.05337.x.

30. Hillyard DZ, Nutt CD, Thomson J, McDonald KJ, Wan RK, Cameron AJ, et al. Statins inhibit nk cell cytotoxicity by membrane raft depletion rather than inhibition of isoprenylation. Atherosclerosis. 2007; 191:319-325

31. Poggi A, Boero S, Musso A, Zocchi MR. Selective role of mevalonate pathway in regulating perforin but not fasl and tnfalpha release in human natural killer cells. PLoS One. 2013; 8:e62932. doi:10.1371/journal.pone.0062932.

32. Raemer PC, Kohl K, Watzl C. Statins inhibit nk-cell cytotoxicity by interfering with Ifa-1-mediated conjugate formation. Eur J Immunol. 2009; 39:1456-1465. doi:10.1002/eji.200838863.

33. Gruenbacher G, Gander H, Nussbaumer O, Nussbaumer W, Rahm A, Thurnher M. II-2 costimulation enables statin-mediated activation of human nk cells, preferentially through a mechanism involving cd56+ dendritic cells. Cancer Res. 2010; 70:9611-9620. doi:10.1158/0008-5472.can-10-1968.

34. Klingemann H, Boissel L, Toneguzzo F. Natural killer cells for immunotherapy - advantages of the nk-92 cell line over blood nk cells. Front Immunol. 2016; 7:91. doi:10.3389/fimmu.2016.00091.

35. Yang L, Shen M, Xu LJ, Yang X, Tsai Y, Keng PC, et al. Enhancing nk cell-mediated cytotoxicity to cisplatin-resistant lung cancer cells via mek/erk signaling inhibition. Sci Rep. 2017; 7:7958. doi:10.1038/s41598-017-08483-z.

36. Johnston JM, Denning G, Moot R, Whitehead D, Shields J, Le Doux JM, et al. High-throughput screening identifies compounds that enhance lentiviral transduction. Gene Therapy. 2014; 21:1008. doi:10.1038/gt.2014.80.

37. Oesterle A, Laufs U, Liao JK. Pleiotropic effects of statins on the cardiovascular system. Circ Res. 2017; 120:229-243. doi:doi:10.1161/CIRCRESAHA.116.308537.

38. Izawa A, Kashima $\mathrm{Y}$, Miura T, Ebisawa S, Kitabayashi H, Yamamoto $\mathrm{H}$, et al. Assessment of lipophilic vs. Hydrophilic statin therapy in acute myocardial infarction - alps-ami study. Circ J. 2015; 79:161-168. doi:10.1253/circj.CJ-14-0877.

39. Bytyci I, Bajraktari G, Bhatt DL, Morgan CJ, Ahmed A, Aronow WS, et al. Hydrophilic vs lipophilic statins in coronary artery disease: A meta-analysis of randomized controlled trials. J Clin Lipidol. 2017; 11:624-637. doi:10.1016/j.jacl.2017.03.003.

40. Law MR, Wald NJ, Rudnicka AR. Quantifying effect of statins on low density lipoprotein cholesterol, ischaemic heart disease, and stroke: Systematic review and meta-analysis. BMJ. 2003; 326:1423. doi:10.1136/bmj.326.7404.1423. 
41. Chan P-C, Lafrenière R, Parsons HG. Lovastatin increases surface low density lipoprotein receptor expression by retarding the receptor internalization rate in proliferating lymphocytes. Biochem Biophys Res Commun. 1997; 235:117-122. doi:https://doi.org/10.1006/bbrc.1997.6736.

42. Tanaka T, Porter CM, Horvath-Arcidiacono JA, Bloom ET. Lipophilic statins suppress cytotoxicity by freshly isolated natural killer cells through modulation of granule exocytosis. Int Immunol. 2007; 19:163-173. doi:10.1093/intimm/dxl133.

43. Bryceson YT, March ME, Ljunggren H-G, Long EO. Activation, coactivation, and costimulation of resting human natural killer cells. Immunol Rev. 2006; 214:73-91. doi:10.1111/j.1600065X.2006.00457.x.

44. Janakiram NB, Mohammed A, Bryant T, Zhang Y, Brewer M, Duff A, et al. Potentiating nk cell activity by combination of rosuvastatin and difluoromethylornithine for effective chemopreventive efficacy against colon cancer. Sci Rep. 2016; 6:37046. doi:10.1038/srep37046.

45. Tatsuno I, Tanaka T, Oeda T, Yasuda T, Kitagawa M, Saito Y, et al. Geranylgeranylpyrophosphate, a metabolite of mevalonate, regulates the cell cycle progression and DNA synthesis in human lymphocytes. Biochem Biophys Res Commun. 1997; 241:376-382. doi:10.1006/bbrc.1997.7825.

46. Bollino D, Webb TJ. Chimeric antigen receptor-engineered natural killer and natural killer t cells for $\begin{array}{llll}\text { cancer immunotherapy. } & \text { Translational } & \text { Research. 2017; } & \text { 187:32-43. }\end{array}$ doi:https://doi.org/10.1016/j.trsl.2017.06.003.

47. Davis HE, Rosinski M, Morgan JR, Yarmush ML. Charged polymers modulate retrovirus transduction via membrane charge neutralization and virus aggregation. Biophysical Journal. 2004; 86:1234-1242. doi:https://doi.org/10.1016/S0006-3495(04)74197-1.

48. De Sanctis JB, Blanca I, Radzioch D, Bianco NE. Expression and function of low-density lipoprotein receptors in cd3-cd16+cd56+ cells: Effect of interleukin 2. Cell Immunol. 1996; 167:18-29. doi:10.1006/cimm.1996.0003.

49. Streltsova MA, Barsov E, Erokhina SA, Kovalenko El. Retroviral gene transfer into primary human nk cells activated by il-2 and k562 feeder cells expressing membrane-bound il-21. J Immunol Methods. 2017; 450:90-94. doi:https://doi.org/10.1016/j.jim.2017.08.003.

50. Colamartino ABL, Lemieux W, Bifsha P, Nicoletti S, Chakravarti N, Remon JS, et al. Efficient and robust nk-cell transduction with baboon envelope pseudotyped lentivector: A major tool for immunotherapy. bioRxiv. 2019; 625285. doi:10.1101/625285.

51. Sutlu T, Nyström S, Gilljam M, Stellan B, Applequist SE, Alici E. Inhibition of intracellular antiviral defense mechanisms augments lentiviral transduction of human natural killer cells: Implications for gene therapy. Human Gene Therapy. 2012; 23:1090-1100. doi:10.1089/hum.2012.080.

52. Suerth JD, Morgan MA, Kloess S, Heckl D, Neudorfl C, Falk CS, et al. Efficient generation of genemodified human natural killer cells via alpharetroviral vectors. J Mol Med (Berl). 2016; 94:83-93. doi:10.1007/s00109-015-1327-6.

53. Lowe E, Truscott L, De Oliveira S. In vitro generation of human nk cells expressing chimeric antigen receptor through differentiation of gene-modified hematopoietic stem cells. Methods Mol Biol. 2016; 1441:241-251. doi:10.1007/978-1-4939-3684-7_20.

54. Li Y, Hermanson DL, Moriarity BS, Kaufman DS. Human ipsc-derived natural killer cells engineered with chimeric antigen receptors enhance anti-tumor activity. Cell Stem Cell. 2018; 23:181-192. doi:10.1016/j.stem.2018.06.002.

55. Arefieva TI, Filatova AY, Potekhina AV, Shchinova AMJB. Immunotropic effects and proposed mechanism of action for 3-hydroxy-3-methylglutaryl-coenzyme a reductase inhibitors (statins). Biochemistry. 2018; 83:874-889. doi:10.1134/s0006297918080023.

56. Chen HH, Lin CL, Yeh CJ, Yeh SY, Kao CH. Statins can increase the risk of herpes zoster infection in asia. Eur J Clin Microbiol Infect Dis. 2015; 34:1451-1458. doi:10.1007/s10096-015-2372-3.

57. Seo S, Boeckh M, Storer BE, Schubert MM, Rotta M, Sandmaier BM, et al. The association between donor and recipient statin use and infections after allogeneic hematopoietic cell transplantation. Bone Marrow Transplant. 2015; 50:444-448. doi:10.1038/bmt.2014.279.

58. del Real G, Jiménez-Baranda S, Mira E, Lacalle RA, Lucas P, Gómez-Moutón C, et al. Statins inhibit hiv-1 infection by down-regulating rho activity. J Exp Med. 2004; 200:541-547. doi:10.1084/jem.20040061.

59. Shrivastava-Ranjan P, Flint M, Bergeron É, McElroy AK, Chatterjee P, Albariño CG, et al. Statins suppress ebola virus infectivity by interfering with glycoprotein processing. mBio. 2018; 9:e0066000618. doi:10.1128/mBio.00660-18.

60. Matthaiou DK, Makris GC, Rafailidis PI, Falagas ME. Statins for infection and sepsis: A systematic review of the clinical evidence. Journal of Antimicrobial Chemotherapy. 2008; 61:774-785. doi:10.1093/jac/dkn019. 
61. Nikolic J, Belot L, Raux H, Legrand P, Gaudin Y, A. Albertini A. Structural basis for the recognition of Idl-receptor family members by vsv glycoprotein. Nat Commun. 2018; 9:1029. doi:10.1038/s41467-018-03432-4.

62. Zhang N, Huang H, Tan B, Wei Y, Xiong Q, Yan Y, et al. Leucine-rich repeat-containing g proteincoupled receptor 4 facilitates vesicular stomatitis virus infection by binding vesicular stomatitis virus glycoprotein. J Biol Chem. 2017; 292:16527-16538

63. Hastie E, Cataldi M, Marriott I, Grdzelishvili VZ. Understanding and altering cell tropism of vesicular stomatitis virus. Virus Research. 2013; 176:16-32. doi:https://doi.org/10.1016/j.virusres.2013.06.003.

64. Bak RO, Porteus MH. Crispr-mediated integration of large gene cassettes using aav donor vectors. Cell reports. 2017; 20:750-756. doi:10.1016/j.celrep.2017.06.064. 


\section{Supplementary data}
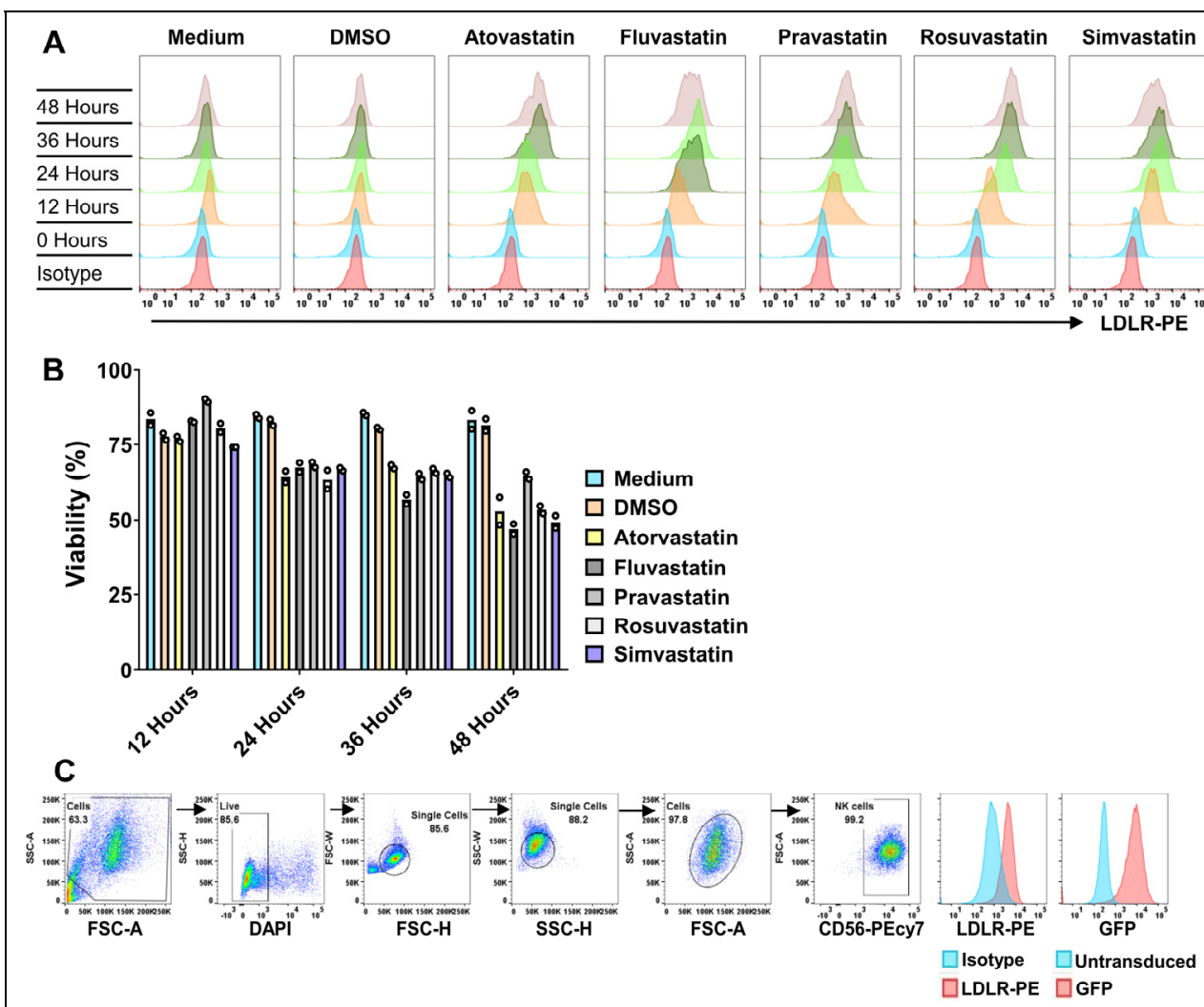

Figure S1. Time course of LDLR expression after statin stimulation.

$0.1 \times 10^{6} \mathrm{cell} / \mathrm{mL}$ NK-92 cells were cultured with $5 \mu \mathrm{M}$ statins for different periods. (A) LDLR expression levels are upregulated by statins in a time-dependent manner Histograms show one representative sample of LDLR expression levels analyzed using flow cytometry. (B) Prolonged culture with statins has a negative impact on NK-92 cell viability. Dead cells were stained with Fixable live/dead Aqua V500. Data shown is one representative of 3 independent assays. (C) Gating strategy of LDLR and GFP expression on NK-92 cells. 


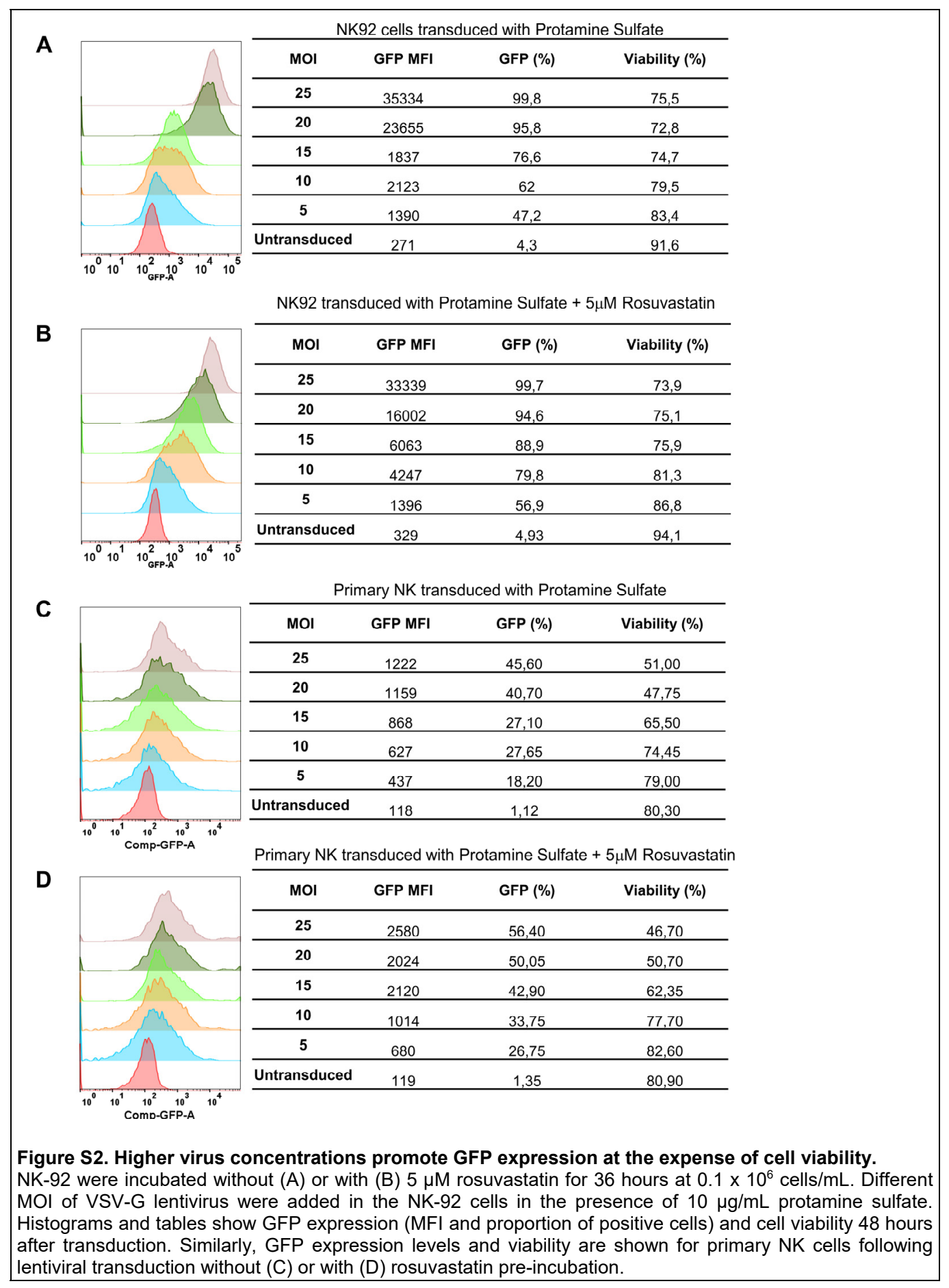


A

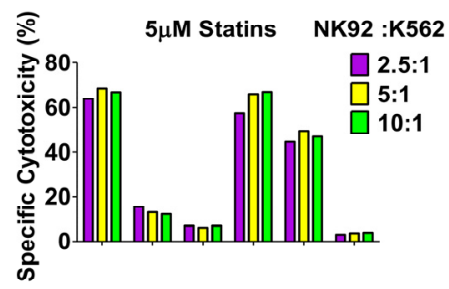

B

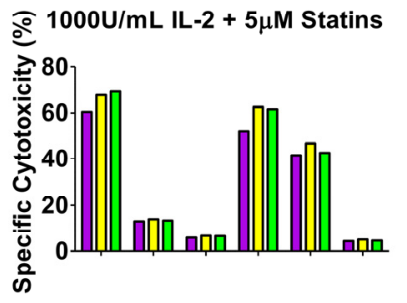

C

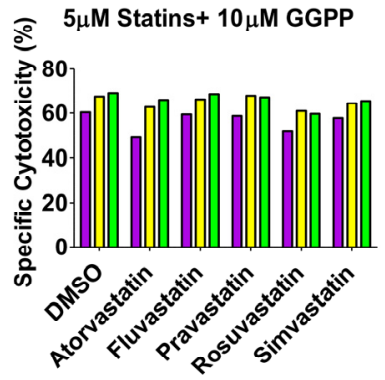

Figure S3. GGPP but not IL-2 can completely reverse the negative impact of statins on the cytotoxic capacity of NK-92 cells.

(A) NK-92 cell cytotoxicity was suppressed by lipophilic statins after 36 hours incubation. However, NK-92 cell cytotoxicity was not changed after pravastatin and not completely be restrained by rosuvastatin. NK-92 cells were seeded at a density of $0.1 \times 10^{6}$ cells $/ \mathrm{mL}$ in 12 well plates for statin stimulation. After removing the supernatant, NK-92 cells were added according to indicated E:T ratio in 96 well-plates. 20,000 K562 cells were seeded per well as target cells. Cytotoxicity assay was performed for 4 hours with different E:T ratios. (B) Statin $(5 \mu \mathrm{M})$ induced inhibition of NK cell cytotoxicity could not be reversed by $1000 \mathrm{U} / \mathrm{mL}$ IL-2. (C) GGPP completely reversed the cytotoxicity suppression of statins on NK-92 cells. Data is shown of one representative assay of 3 independent assays as mean cytotoxicity. 
A

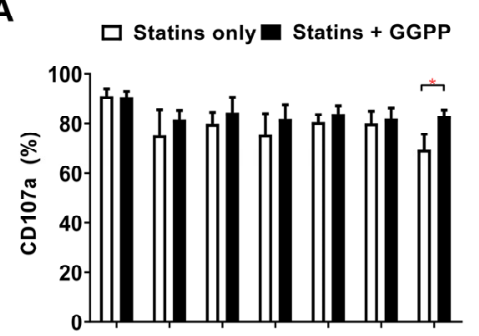

B

C

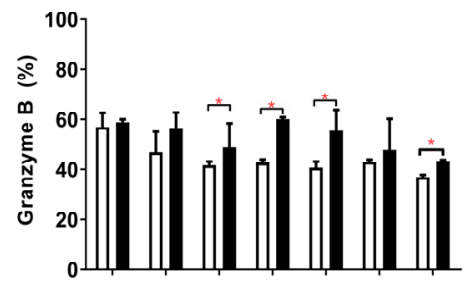

D
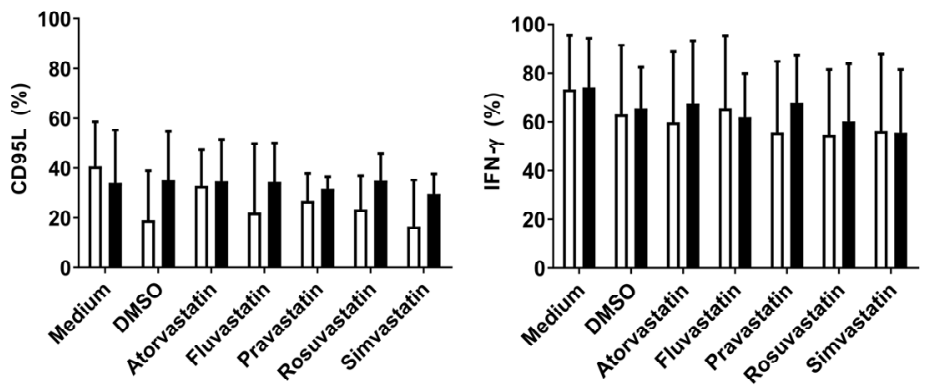

E

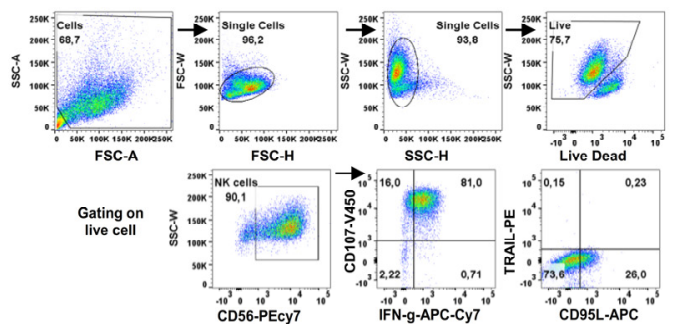

Figure S4. Statins have a negative effect on CD107a and granzyme B expression, which can be reversed using GGPP.

$0.1 \times 10^{6} \mathrm{NK}-92$ cells were cultured in $1 \mathrm{~mL}$ with $5 \mu \mathrm{M}$ statins for 36 hours. Medium was replaced with medium containing CD107a - V450 antibody, and PMA $(20 \mathrm{ng} / \mathrm{mL})$ plus ionomycin $(1 \mu \mathrm{g} / \mathrm{mL})$. After 1 hour preincubation, $10 \mu \mathrm{g} / \mathrm{mL}$ brefeldin A was added. Cells were stained with surface markers (CD56 and CD95L), and subsequently fixed and permeabilized, followed by IFN-y intracellular staining. CD107a (A), Granzyme B (B), CD95L (C) and IFN-Y (C) expression in NK-92 were analyzed by flow cytometry. Compared with medium and DMSO, only simvastatin down-regulated CD107a, but was reversed by GGPP. All the statins inhibit the granzyme B, except for rosuvastatin. Fortunately, this negative effect was reversed by adding $10 \mu \mathrm{M} \mathrm{GGPP}$. (E) Gating strategy of CD107a, CD95L and IFN-y on NK-92. Data is shown as mean \pm SD from $N=3$ independent experiments performed at different times. Data analysis was performed using a pair signed-rank test between the statins only group and the statins plus GGPP group. 


\section{4 | Chapter 3}
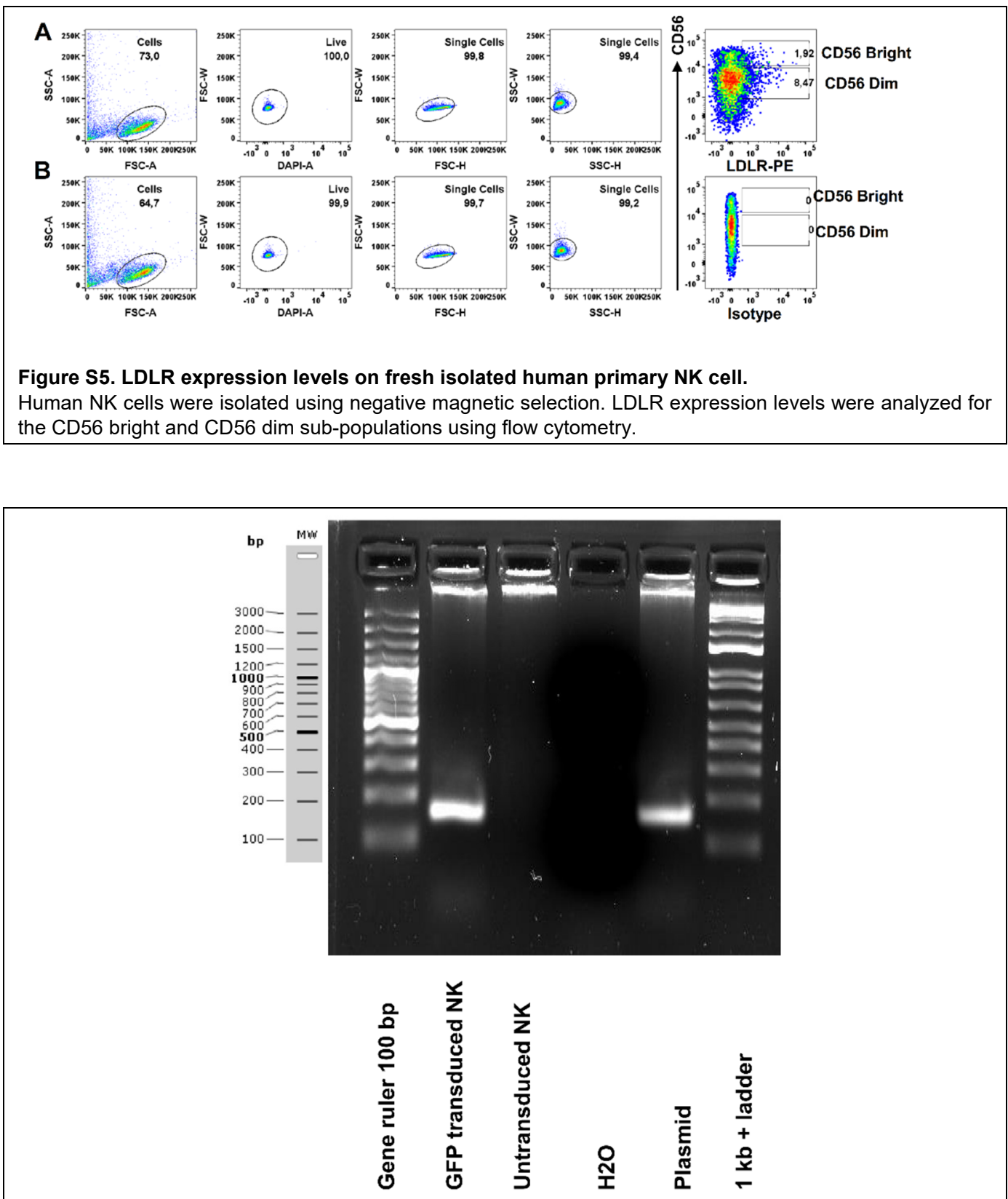

Figure S6. Lentiviral integration in human primary NK cells.

Genomic DNA was extracted 48 hours after VSV-G lentiviral transduction of human primary NK cells. One pair of primer specific amply the GFP fragment with a size at $157 \mathrm{bp}$. PCR product was running on $1.2 \%$ agarose TAE gel ( $70 \mathrm{~V}, 45$ minutes). A plasmid encoding GFP was used as positive control, $\mathrm{H} 2 \mathrm{O}$ is used as the 'no template' control. Untransduced NK cells were used as a negative control. 


\section{Supplemental Methods}

\section{CD107a degranulation assay}

To evaluate NK cell activation by tumor cells or after stimulated with Phorbol 12myristate 13-acetate (PMA, Sigma Aldrich) plus ionomycin (Sigma Aldrich), CD107a expression on NK cells was analyzed through flow cytometry. NK cells were incubated with PMA $(20 \mathrm{ng} / \mathrm{mL})$ plus ionomycin $(1 \mu \mathrm{g} / \mathrm{mL})$ with $2 \mathrm{~L}$ anti-CD107a-Horizon V450 (H4A3, BD Biosciences) in a 96-well plate. After 1 hour of co-culture, $10 \mu \mathrm{g} / \mathrm{mL}$ Brefeldin A (BFA, BD Biosciences) was added. After another 3 hours, the plate was placed on ice to stop the reaction. NK cells were further stained with anti-human CD3 and CD56 antibodies conjugated with appropriate fluorophores surface markers as described above.

\section{Intracellular staining}

For intracellular staining of interferon-gamma (IFN- $\gamma$, cells were stimulated with PMA $(20 \mathrm{ng} / \mathrm{mL})$ plus ionomycin $(1 \mu \mathrm{g} / \mathrm{mL})$ and $10 \mu \mathrm{g} / \mathrm{mL}$ BFA in the culture medium for 4 hours. Then cells were harvested, washed, and stained with fluorochrome-conjugated anti human CD3 and CD56 surface monoclonal antibodies for 30 minutes at $4^{\circ} \mathrm{C}$. Cells were then washed twice and treated with fixation buffer (eBiosciense, Thermo Scientific) for 15 minutes at room temperature. After washing, cells were permeabilized in permeabilization buffer for 30 minutes at $4^{\circ} \mathrm{C}$ degrees. After washing, fixation and permeabilization, cells were stained with fluorochrome-conjugated anti human IFN- $\gamma$ antibody (Clone: 45-15, Miltenyi) anti human Granzyme B (Clone REA226, Miltenyi Biotec). After 2 more washes, the cells were analyzed by FACS Canto II (BD Biosciences).

\section{Genomic extraction and PCR}

48 hours after transduction, $0.5 \times 10^{6}$ primary NK cells were harvested. Primary genomic DNA was isolated using Lucigen QuickExtract ${ }^{\mathrm{TM}}$ DNA Extraction Solution (QE0905T, Lucigen Corporation, Wisconsin, USA) as published elsewhere [64]. The GFP construct was amplified using PrimeSTAR HS polymerase kit (R010A, Takara Bio) and the following primers: GFP-forward gtgatgggctacggcttctacc and GFP-reverse primer cctcgtagcggtagctgaag. 
86 | Chapter 3 


\section{Chapter 4}

Chimeric Antigen Receptor Natural Killer (CAR-NK) cell design and engineering for cancer therapy

Gong Y, Klein Wolterink RGJ, Wang J, Bos GMJ, Germeraad WTV. J Hematol Oncol. 2021; 14:73. doi:10.1186/s13045-021-01083-5. 


\section{Abstract}

Due to their efficient recognition and lysis of malignant cells, Natural Killer (NK) cells are considered as specialized immune cells that can be genetically modified to obtain capable effector cells for adoptive cellular treatment of cancer patients. However, biological and technical hurdles related to gene delivery into NK cells have dramatically restrained progress. Recent technological advancements, including improved cell expansion techniques, chimeric antigen receptors (CAR), CRISPR/Cas9 gene editing and enhanced viral transduction and electroporation have endowed comprehensive generation and characterization of genetically modified NK cells. These promising developments assist scientists and physicians to design better applications of NK cells in clinical therapy. Notably, redirecting NK cells using CARs holds important promise for cancer immunotherapy. Various pre-clinical and a limited number of clinical studies using CAR-NK cells show promising results: efficient elimination of target cells without side effects, such as cytokine release syndrome and neurotoxicity which are seen in CAR-T therapies. In this review, we focus on the details of CAR-NK technology, including the design of efficient and safe CAR constructs and associated NK cell engineering techniques: the vehicles to deliver the CAR-containing transgene, detection methods for CARs, as well as NK cell sources and NK cell expansion. We summarize the current CAR-NK cell literature and include valuable lessons learned from the CAR-T cell field. This review also provides an outlook on how these approaches may transform current clinical products and protocols for cancer treatment.

\section{Keywords:}

Cancer Immunotherapy; Adoptive Cell Therapy; Chimeric Antigen Receptor; Natural Killer cells; Genetic Modification. 


\section{Introduction}

The human immune system has long been recognized as an important pillar of anticancer responses [1,2]. As tumor cells originate from normal tissue, one of the main struggles in the design of anti-cancer therapies is the design of treatments that specifically target cancerous cells without affecting normal tissue. Ideally, these therapies have a strong potency against a particular malignancy that is characterized by rapid proliferation, resistance to apoptosis, and continuously changes through mutations and adaptations to the environment [3]. Recently, adoptive immune cell therapy (ACT) has shown promising clinical outcomes in patients with hematologic malignancies but remains difficult in treatment of solid tumors [4]. Unlike dispersed hematologic malignancies, solid tumors - especially late-stage - exhibit an inhibitory tumor microenvironment due to hypoxia, a low $\mathrm{pH}$, and presence of suppressive cytokines, lactate, prostaglandins, and others. These factors circumvent the ability of effector cells like NK cells to infiltrate and function in an optimal fashion [5]. Development of ACT should always be developed to overcome this induced tolerant state.

In ACT, cells are collected from peripheral blood of autologous or allogeneic donors and are genetically engineered with a chimeric antigen receptor (CAR) that recognizing tumor-specific proteins on the cell surface. Often, this procedure also includes an ex vivo expansion step to obtain sufficient cell numbers [6,7]. At this moment, the most successful form of ACT comprises of a CD19 CAR-T cell that cures up to $90 \%$ of patients with advanced refractory acute lymphoblastic leukemia (ALL). Here, complete remissions are attained within 4 weeks of treatment, as evident from several trials across multiple institutions $[8,9]$.

A CAR is composed of three domains: an ectodomain, the transmembrane region and a cytoplasmic activation tail $[6,10]$. The ectodomain contains a single chain variable fragment (scFv), usually derived from antibodies that provides the ability to specifically recognize tumor antigens expressed on cancerous cells [11]. The transmembrane domain anchors the CAR structure on the effector cell membrane [12]. Once the CAR recognizes and gets triggered by its specific antigen, the intracellular activation domain(s) of the CAR will signal, resulting in downstream processes that facilitate the killing of target cells $[13,14]$. Numerous immune effector cells, like T cells, $\gamma \delta$ T cells, Natural Killer (NK) cells, NKT cells, and macrophages have been equipped with a CAR, and were shown to be able to mediate anti-cancer responses in pre-clinical studies and clinical trials $[4,15,16]$. In 2017, the US Food and Drug Administration (FDA) approved the first two CAR-T treatments for CD19+ hematologic malignancies (axicabtagene ciloleucel and tisagenlecleucel) $[1,17]$.

NK cells are innate immune effector cell that can rapidly identify and kill abnormal cells, virally infected cells and tumor cells [18]. In contrast to T cells, NK cells are lymphocytes that lack antigen-specific receptors, while abundantly expressing neural cell adhesion molecule (NCAM; also known as CD56). The unique mechanism of NK to distinguish pathologic cells from normal tissue cells is determined by the combination of surface stimulatory and inhibitory receptors that recognize a wide range of ligands on target cells [19]. T cells recognize peptide in the $\mathrm{MHC}$ (in human called human leukocyte antigen, HLA) molecules on an antigen presenting cell (APC) and after receiving the proper danger signals, APC activate T cells that can kill MHC class I expressing tumor cells. However, tumor cells or stressed (e.g., virus-infected) cells often exhibit reduced or no expression of MHC class I molecules, and thereby become susceptible to NK cell 
elimination [20]. MHC class I molecules bind a suite of inhibitory killer cell immunoglobulin-like receptors (KIRs). The KIR cluster restrains NK cell activity and thereby prevents the damage to normal "self"-cells [21]. During NK cell development, the interaction between KIRs and autologous $\mathrm{MHC}$ molecules provides essential signals for NK cell maturation and contributes to the acquisition of functional competency, a process termed NK cell licensing [15]. This process is part of a sophisticated mechanism known as NK cell education, in which NK cells obtain functional competence and adapt to the host where they develop [21,22]. NK cells, unlike T cells, can also become activated after antibody binding to the CD16 surface receptor. When an antibody binds to a specific tumor antigen, this complex is bound via the Fc tail to CD16 molecules on NK cells that become activated and will eliminate the tumor cells.

An interesting property of mature NK cells for adoptive cell therapy is that they can be transplanted into a new surrounding with different $\mathrm{MHC}$ expression patterns without losing their function $[23,24]$. In great contrast to T lymphocytes, NK cells do not induce graft-versus-host disease but rather play a regulatory role in most cases (reviewed in [25]). With the development of genetic modification technologies, NK cells have been demonstrated they can be further tailored, including the introduction of CARs and knockout of inhibitory genes [26]. Using such techniques, NK cells from patients with hematologic malignancies can swiftly kill autologous tumor cells that were previously resistant to killing by the same NK cells without a CAR $[11,27]$. Preclinical studies with CAR-NK cells unraveled in vivo activity similar to that of CAR-T cells in xenograft murine models. Interestingly, the CAR-NK groups show less cytokine release and better overall survival rates $[28,29]$. A first CAR-NK study in men showed a promising anti-tumor response without the development of major toxic effects, such as CRS and GVHD [30]. These encouraging results pave the way for further development of CAR-NK as an attractive modality for cancer therapy [11].

In this review, we summarize information from pre-clinical and clinical studies, reporting on 72 CAR-NK cell line and 35 primary CAR-NK cell investigations. We try to interpret the paradigm for CAR-NK by focusing the design of a CAR and engineering of NK cells. We describe in detail the structure of the CAR, several detection methods of the CAR on the NK cells, the ideal NK cell source for CAR-NK therapy and the techniques for ex vivo expansion of NK cells. We also discuss the approaches to deliver the CAR-containing transgene to NK cells and the methods that have been used to enhance the transduction efficiency. Finally, we will provide a comprehensive outlook on how future CAR-NK-based therapies can be used to eradicate cancer.

\section{A global overview of current CAR-NK cell studies}

We found 72 CAR-NK cell line studies and 35 primary CAR-NK preclinical studies based on "Chimerical antigen receptor and Natural killer cells" from PubMed and Global data $^{\circledR}$ (until March 2021). All these investigations introduce an external antigen binding motif derived from a tumor-targeting monoclonal antibody (moAb) into the cells. In (Table 1 and Table 2), we provide an overview of these studies using cell lines and primary NK cells, respectively. Due to space constraints, further details on the CAR design and study are made available online as interactive tables on www.carnkreview.com and are provided as (Supplemental Table 1 and Supplemental Table 2). 


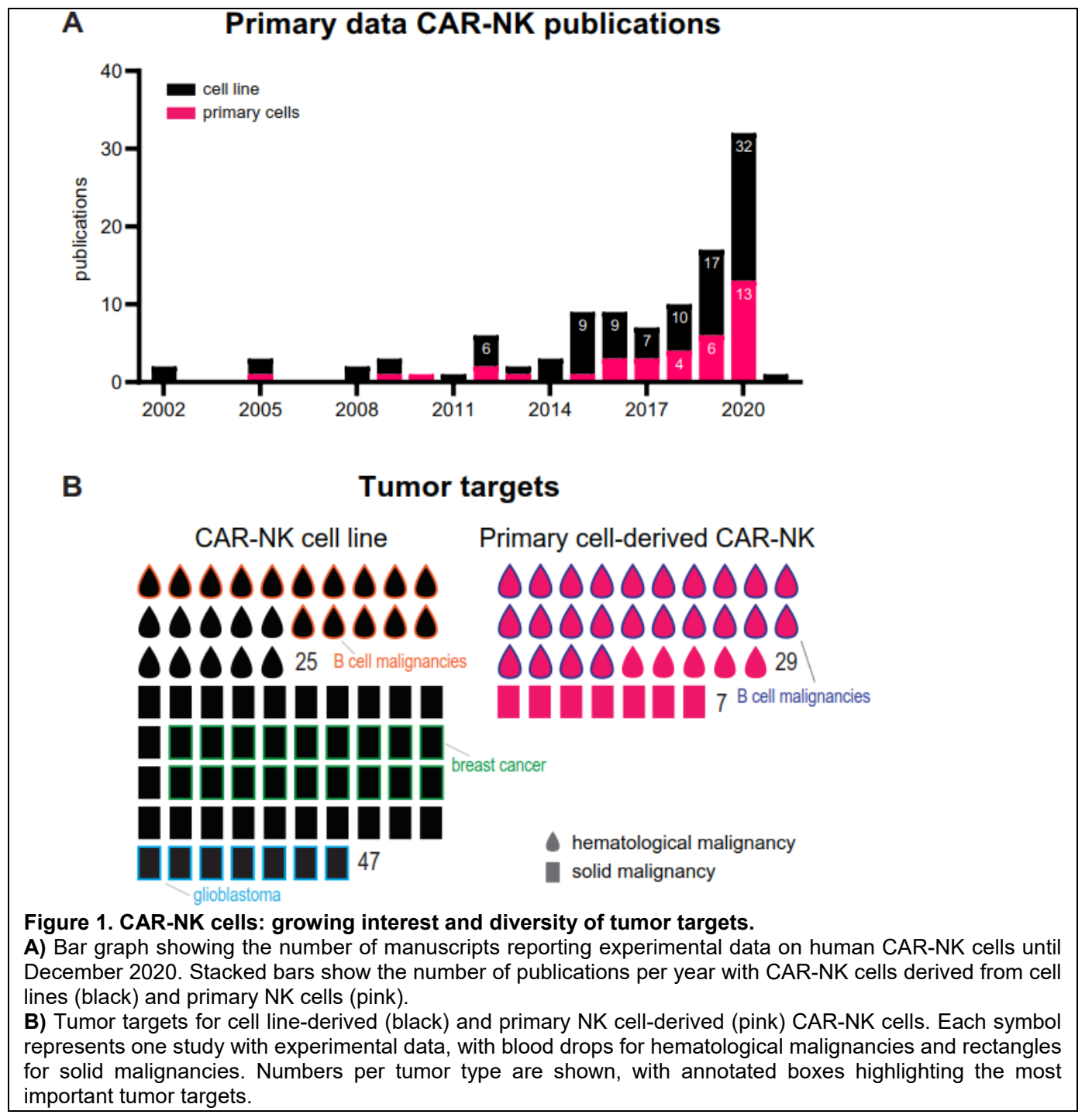

The number of CAR-NK pre-clinical studies is increasing year by year, describing both CAR-NK cell lines as well as primary CAR-NK cells (Figure 1A). In this review, we will zoom in on the various CAR elements and the techniques required generating CARNK cells for clinical applications. At first glance, the overview of the current CAR-NK studies already shows interesting trends. In CAR-NK cell line studies, Her2 (expressed on a subset of breast cancer cells) is the most used target for solid tumors, while the CD19 antigen (B cell malignancies) is the most popular in hematological cancers (Figure 1B). Of the primary NK cell studies, $65 \%$ use primary CAR-NK cells investigating B cell malignancies with CD19 as the most favorite target (Figure 1B). Interestingly, the number of solid tumor CAR-NK cell lines studies is over 2 times higher than of hematological malignancies. In the following chapters, we will discuss the various components of that make up a successful CAR-NK cell. 


\section{CAR Structure design}

Functional CAR molecules expressed on NK cells consist of three parts: the ectodomain, transmembrane region and the endodomain (Figure 2A). The ectodomain is made up of a signal peptide, the single chain fragment variant (scFv) with a linker between the heavy chain and light chain and the hinge region that connects this structure to the transmembrane region. This latter region docks the CAR molecule to the cell membrane. It also connects intracellularly to the endodomain that encompasses the activating signals. The combination of these building blocks, together with regulatory elements that are situated outside the open reading frame (e.g. promotor), will determine the efficacy of the CAR. Successful CAR design is achieved by a combination of careful in silico design and functional testing. In this section, we will discuss the various building blocks of a CAR in detail and will summarize the current knowledge of successful combinations of these components. This information, often combined with software that assists in the analysis of expression levels, protein folding and spatial confirmation resulting from a certain DNA and protein sequence [31], will aid in successful CAR design. Before discussing CAR design, we will focus on the vector that carries the transgene and facilitates the insertion into the effector cell.

\subsection{Vector backbone and promoter}

The vector backbone contains all elements needed for expression of the CAR such as the promoter, the polyadenine signal and transcriptional regulation fragments.

As promoters initiate CAR transcription, the choice for a certain promoter has a direct influence on expression levels of the transgene. Furthermore, Allan et al. have recently reported that the size of the promoter influences the viral transduction efficiency and subsequent GFP expression in a negative correlation [32]. In their study, the shorter EFS, PGK, SV40 and CMV promotors are good promoters that can maintain the GFP expression for 17 days [32]. CAR genes are usually introduced using viral plasmids with an exogenous promoter. Currently, there is only one comparison of different promotors on CAR expression and function in NK cell lines published, while no comparative data is available for primary NK cells [33]. From the single report on CAR-NK cells, no definitive statements can be made about the best promotor for CAR-NK cells [33]. In primary T cells, various comparisons of the effect of promoters on CAR expression levels were performed. One study shows that the EF1a promotor induced higher CAR expression levels compared to other commonly used promotors, such as CMV, UbiC and PGK [34]. However, another study showed that the MSCV outperformed both EF1a and PGK in terms of expression levels and CAR expression stability [35], however, the promotor efficacy may be influenced by the scFv sequence itself [36] (see section 2.3). Given these and other studies [37] that report different optimal promoters, it is important to determine the optimal promoter for a given construct (that may also contain additional elements that influence expression levels, such as reporters) and viral vector. The latter is important, since the choice of a promoter also influences the virus titer. For instance, one study reports that the CMV promoter sequence yielded higher virus titers compared to RSV promoter sequences when located within the SIN 5' LTR lentiviral vector [38]. Besides baseline CAR expression in the immune cell, the dynamic ubiquitination, downregulation and re-expression kinetics following antigen exposure will have large impact on the efficacy of CAR cells [39]. 
Compared with the retroviral LTR promoter-based CAR that integrates "randomly", including in potential oncogenic sites, it may be desirable to make use of site directed insertion into a target cell specific promotor area. An interesting example is the use of the endogenous TRAC promotor where the CAR gene was deliberately integrated into the TCR alpha chain gene locus [40]. This concept was already introduced as a way to avoid graft-versus-host disease in T cell-based adoptive cell therapy [41]. Eyquem and colleagues took this a step further by demonstrating that CAR transduced lymphocytes integrated in the TRAC locus persist longer in murine tumor models [40]. In addition, more effector cells infiltrated into the tumor and exhibited a less exhausted cell phenotype [40]. TRAC-CAR-T outperformed both conventional retroviral transduced CAR T cells (RV-CAR) as well as T cells with the CAR inserted in the $\beta 2 \mathrm{M}$ locus $(\beta 2 \mathrm{M}$ CAR) in an in vivo mouse model, based on tumor eradication and expression of exhaustion markers, including PD1, TIM3, and LAG3 [40]. In addition, TRAC-CAR increased CAR expression levels after antigen stimulation, while CARs driven by an exogenous promotor showed important downregulation. Although this CAR expression under an endogenous NK cell-specific promoter has not been reported, but GFP or HA epitopes have successfully been expressed in primary NK cells by CRISPR/Cas9mediated knock-in into the CLTA [42], AAVS1 [43], CD96, ACTB and RAB11A gene loci [26]. Thus, the use of endogenous promotors should also be further explored in the CARNK cell field.

Current reports on CAR-NK cells show a wide variety of promoters being used for driving CAR expression, both for cell line-derived and primary NK cells. In both primary CAR-NK and CAR-NK cell lines, viral promoters (CMV, MPSV, MMLV, SFFV, etc.) are more frequently used to generate CAR-NK than constitutively active promoters, such as EF1a, CMV and PGK ( $80 \%$ and $78 \%$ for primary and cell line CAR-NK, respectively; Supplemental Table 1 and Supplemental Table 2).

\subsection{Signal peptide for CAR-NK}

The CAR sequence starts with a short signal peptide (SP). These are short peptides located at the $\mathrm{N}$-terminus of proteins, carrying information for protein post-translational assembly in the endoplasmic reticulum (ER) and Golgi organelle and expression on the membrane [44]. The final CAR protein on NK cells will be a type I transmembrane protein that anchors on the cell membrane. CAR, like many other proteins, are also secreted by immune cells via the co-translational translocation pathway [45]. In eukaryotic cells, SP located at the $\mathrm{N}$-terminus of nascent proteins are identified by the signal recognition particle (SRP) while the protein is still translating in the ribosome. After the nascent protein crosses the ER membrane, the signal peptide is cleaved off by a signal peptide peptidase (SPP), then protein assembly and folding will commence [46].

There is enormous heterogeneity in signal peptides, which directly translates into different levels of protein secretion efficiency. For both CAR-NK and CAR-T cells, we did not find comparative studies that determined the optimal signal peptide for CAR expression and function. At present, CD8a SP is the most used peptide sequence for primary NK (16\%, with data missing in $71 \%$ of the studies; Supplemental Table 2$)$ and immunoglobulin heavy or light chain SP for NK cell lines (29\%) (Supplemental Table 1). While the SP sequence is likely to influence CAR expression levels, there is currently no data to support claims about the optimal SP for CAR-NK generation. 


\subsection{Single chain fragment variant (scFv)}

The single chain fragment variant (scFv), a fusion protein of the variable regions of the heavy and light chain of an antibody, is the tumor antigen-binding domain of the CAR. Different ScFv could bind to different epitopes of the same protein and therefore, this domain will determine both the specificity and the function of the CAR-NK cell [47]. For instance, targeting Mucin 1 (MUC1) which is a glycosylated, abundant protein, many antibodies have been described and various ScFv's have been created in CAR constructs $[48,49]$. Diverse scFvs lead to various anti-tumor capacities but also distinct side-effects, such as binding to normal tissue. Therefore, the target of a CAR needs to be selected carefully to be specific for the tumor-associated antigen, while at the same time not recognizing self-antigens that could induce severe side-effects [1].

However, the numerous efforts undertaken to find a suitable target on solid tumors illustrate how difficult the process of scFv selection can be. This is largely due to the expression of most so-called "tumor-associated antigens" on both tumors and - often at lower levels - in healthy tissue. There already have been reports of the potential damaging effects of CAR-engineered T cells, such as the 4D5-scFv anti-ERBB2 [50] and G250-scFv anti-CAIX [51].

As the scFv is not a naturally occurring form of an antibody, it is always synthesized, and therefore the order of the heavy and light chain is artificially determined. Thus far, most groups prefer a VH-VL orientation over VL-VH for CAR-NK design (Supplemental Table 1 and Supplemental Table 2). Fujiwara et al. demonstrated that the order of $\mathrm{H}$ and $L$ chain did not influence the expression level of anti-KDR CAR expression on $T$ cells [36]. In addition, scFv performance in terms of antigen specificity and association constant in a CAR is sometimes found to be lower compared to its originating antibody, mostly due to the changed connectivity of the $\mathrm{VL}$ and $\mathrm{VH}$ domains. This may also have implications for the dynamics and relative orientation of the two chains. Computationalassisted design of a scFv may help in the development of functional scFv by analyzing the amino acid sequence of the CDR region of the VH and VL, and predicting the interaction of the scFv with its target [52]. Interestingly, VH and VL chains from different antibodies against the same epitope can also be mixed, sometimes leading to increased CAR affinity [53].

The importance of scFv design was underlined by a study that shows distinct scFvs targeting the same antigen may yield very different results in terms of CAR expression [36]. In this study, CAR-T cells that expressed functional CARs were detected, but also CAR-T cells with no or very low levels of antigen binding. Here, changes in the CDR region accounted for the observed differences, while there were no effects due to a specific $\mathrm{VH} / \mathrm{VL}$ order [36]. Another example was published very recently and showed that from the 8 differently derived scFvs specific for CD19 when lentiviral transduced as CAR into the NK cell line KHYG-1, only two of them were efficiently expressed on the cell surface and showed improved cytotoxicity towards CD19+ tumor cell lines [54]. However, whether this was due to the specific sequence of the scFv as the CDR3 regions do vary, or a different spacer and transmembrane domain remains to be elucidated [54].

In addition, immune cells can be equipped with multiple scFv, thus broadening the antigen recognition capacity of a CAR effector cell. Here, there are various options: CARs can be transduced with vectors with bi-cistronic elements, inducing expression of two CAR constructs that may or may not have the same intracellular structure. In addition, two scFvs can be fused together in one construct, generating a tandem scFv or "single 
stalk" CAR. While these techniques have been employed for the generation of CAR-T cells [55], we are not aware of such attempts for generating CAR-NK cells.

Most current clinical CAR $T$ cell trials have utilized $\mathrm{scFv}$ derived from murine antibodies, thereby increasing the risk for an anti-mouse IgG cell host-versus-graft disease, resulting in toxicity or shortened persistence of CAR-bearing cells [6]. This issue may be attenuated by humanizing murine scFv or by deriving scFv from fully human antibodies. In previous studies that employed murine scFv-based anti-CD19-CAR or anti-CD5-CAR, the NK-92 cells exhibited strong anti-tumor activity to ALL malignant cells both ex vivo and in a mouse model [56,57]. An anti-GD2-CAR with humanized scFv showed similar expression levels as its murine counterpart, while increasing cytokine release and $\mathrm{T}$ cell proliferation [58]. Unfortunately, owing to the chimeric character of these CAR receptors, even humanized ScFv constructs still may induce a host antiidiotype immune responses [59]. Fortunately, in the limited number of CAR-NK clinical trials so far, no major side-effects related to the anti-CAR immune responses were found [30]. Above all, from these studies, both murine and humanized scFv derived CAR-NK exhibit robust cytotoxicity against antigen-expressing tumor cells both in vitro and in vivo.

\subsection{Linkers}

The linker between the heavy and light chain contributes to the conformation of the scFv and therefore partly determines how well a CAR recognizes the target epitope. Linkers that are too short induce the formation of multimers, because they prevent association of the $\mathrm{VH}$ and $\mathrm{VL}$ domains. Too long domains, on the other hand, may induce proteolysis or weak association between the VH and VL domains [6]. For CAR-NK cells, multimers of the pentapeptide GGGGS (glycine-serine) are most widely used, often as a 15-mer (G4S)3. Another linker designed to enhance proteolytic stability is the Whitlow "218" linker: GSTGSGSKPGSGEGSTKG. In one study, this linker enhanced scFv affinity, possibly altering CAR-NK cell function as well [60]. Fujiwara et al. compared G4S and 218 linkers, but, in their anti-KDR-CAR-T, there were no expressional and functional differences between (G4S)5, (G4S)3 and 218 linkers [36].

Currently, there is insufficient data available to draw conclusions about the best linker for CAR. We found that 18 studies used G4S linkers, whereas 2 publications applied 218 linkers among the CAR-NK cell lines (Supplemental Table 1). While most primary CAR-NK reports did not provide specifics on the linker, all 4 studies that provided these details used G4S linkers to generate CAR-NK (Supplemental Table 2).

\subsection{Hinge region (CD8 $\alpha$, Ig CH2CH3)}

The hinge region, also referred to as a spacer, is the extracellular structural region of the CAR that connects the ScFv units to the transmembrane domain. These spacers generally maintain the stability required for robust CAR expression and activity in effector cells. The hinge region also supplies flexibility to allow easy access to the targeted antigen. The majority of CAR-NK constructs use derivatives of CD8a or CD28 extracellular domains or immunoglobulin G (IgG)-based hinges (Figure 2B). Accumulating evidence shows that the type and length of the spacer importantly influence the functional activity of the CAR [6]. As no specific data is available for CARNK cells, this part of information is all derived from the CAR-T field, and it remains to be shown if this can be directly translated to CAR-NK design.

In a direct comparison between the CD28 and CD8 $\alpha$ hinge domains, it was found that CD28 hinge domains are more likely to promote dimerization of CAR molecules [61]. 
As a result, the activation stimulus derived from CD28 hinge-bearing CARs is stronger [61]. While this may be beneficial, this could also induce cytokine release syndrome, one of the most severe side-effects of CAR-based therapy. Indeed, in a clinical study aiming to improve the safety of anti-CD19-CAR-T therapy, it was found that an optimized CD8 $\alpha$ hinge region, together with other modifications, importantly improved the safety profile of the therapy [31].

Immunoglobulin G-based hinges are also widely used in CAR constructs. A major advantage of an IgG-based hinge region is the flexibility of the construct that is typically composed of IgG1 or IgG4 and the $\mathrm{CH}_{2} / \mathrm{CH} 3$ domains of IgG Fc. The use of the latter domains is optional, and thus allows for modulation of the length of the spacer. While a wide range of spacer lengths will accommodate antigen recognition, studies have found that shorter spacers were associated with increased cytokine production, CAR-T cell proliferation and better persistence and antitumor effects in vivo [62]. For CAR-NK cells, we found that most studies employ a CD8 $\alpha$ hinge region, both in primary NK cell $(16 / 32)$ and CAR-NK cell lines (41/73) (Figure 2B). Other spacers, such as CD28, IgG FC domains and DAP12 were also used in CAR-NK.

\subsection{Transmembrane domain (CD3, CD8, CD28, NKG2D, 2B4)}

The transmembrane (TM) domain connects the ectodomain of the CAR to the intracellular activation signaling domains and docks the receptor to the NK cell membrane. The most commonly used TM parts of CAR-NK have been adapted from CD33, CD8 and CD28, but others (e.g. NKG2D, 2B4, DNAM1) have been explored as well (Figure 2C). The choice of the TM domain was shown to influence the functionality of the CAR construct in the extent of cell activation. The TM domain of CD28, CD16, NKp44, NKp46, NKG2D, DNAM-1 and 2B4 have been used to screen for CAR function using the NK-92 cell line. Interestingly, the TM from molecules typically expressed on NK cells, like DNAM-1, 2B4 and NKG2D lead to more CD107a degranulation and higher cytotoxicity. Thus, the specific source of the TM will determine the activity of CAR-NK [28].

One important aspect of the TM domain is that the optimal TM region should follow the natural orientation ( $\mathrm{N}$ - to $\mathrm{C}$-terminal order) of the protein from the transmembrane protein on the T cells or NK cells [63]. In this paper, Guo et al. addressed the use of the NK-native molecule NKG2D as a TM region and activator signal for NK-92 cells [63]. NKG2D is a powerful activator of NK cells and thus constitutes a suitable backbone for NK-focused CAR design. However, natural NKG2D has the transmembrane region in the $\mathrm{C}$ - to N-terminal order with a short cytoplasmic tail. Interestingly, the use of a signal peptide to forcibly reverse the NKG2D TM region to the $\mathrm{N}$ - to $\mathrm{C}$-terminal order, showed in combination with 2B4 and DAP10 the strongest cytotoxic effects in iPSC-derived CARNK cells [28]. But whether this is really due to the reversed order alone cannot be concluded as the Kaufmann group focused on identifying the best combination of intracellular signaling domains.

These CAR-NK studies underline that it is important to consider the TM region in its function as a linker to the intracellular activation signal, as the type of TM may influence signal transduction and CAR dimerization. In CAR-T cells, CD28-derived TM domain was shown to promote activation-induced cell death (AICD) and cytokine production, whereas CARs with a CD8a-derived TM assisted in CAR dimerization with endogenous TCRs, leading to first activation signal of T cells [61]. At this moment, CD8 $\alpha$ and CD28dervied TM are most popular in primary CAR-NK cells, while CD28 was the preferred TM region for CAR-NK cell lines (Figure $2 \mathrm{C}$ ). 


\subsection{Activation signal for CAR-NK}

CARs are often identified by their respective generation. The number of intracellular activating signals determines this "generation": first generation CARs have one activating signal, second generation CARs have two, and so forth (Figure 2A and Figure 2D).

The activating domains are responsible for the activation of the NK cell upon recognition of the target antigen. In contrast to T lymphocytes, NK cells use a variety of different, non-rearranging receptors for activation. This also includes a large variety of cytokine receptors that are important regulators of development, maturation and activation of NK cells [64]. Most of these receptors share common adapter molecules and signaling pathways. For instance, NKp30 and CD16 both signal via CD3ろ [65]. The cytokines IL-2, IL-7, IL-12, IL-15, IL-18, IL-21, IL-27, and IFN- $\alpha / \beta$ signal via the highly conserved JAK/STAT pathway [64]. Thus, even though the first activation signal in T cells is provided through a clonally rearranged antigen receptor, many of the downstream signaling pathways are shared between T and NK cells. Therefore, some insights from the CAR-T field also apply to CAR-NK cells. At the same time, it is important to realize that CAR-based signaling differs significantly from canonical activation pathways. For instance, in normal T lymphocytes, activation depends on the stabilization of multimolecular complexes (TCR-peptide-MHC with co-receptors) and recruitment of co-stimulatory molecules, whereas these signals are provided 'in line' in CAR-equipped cells. This has direct consequences for the temporal availability of the various activating molecules involved. We have only just begun to grasp the importance of these differences between canonical signaling and CAR signaling. The findings from the CART field (summarized by Lindner and colleagues [66]) will again serve as an important starting point for studying these differences in CAR-NK cells as well.

First generation CAR-NK cells, like CAR-T cells, only contain the CD3 3 signal. The second and third generation CAR-NK bear one and two additional co-stimulatory signals, respectively. The costimulatory molecules are usually derived from the CD28 family (including CD28 and ICOS), the tumor necrosis factor receptor (TNFR) family of genes (including 4-1BB, OX40 and CD27) or signaling lymphocytic activation molecule (SLAM)related receptor family (comprising 2B4) [67]. To these activating domains, safety switches can be added to quickly eliminate the infused CAR-containing cells in the case of adverse reactions occur, such as the severe cytokines release storm (CRS) $[30,68]$ (this aspect will be further discussed in section 8.4). In addition to this example, other modifications to the effector cells have been developed that improve persistence, enhance tumor activity, prevent antigen escape, allow for control of CAR expression or combinations of these (Table 3). An in-depth discussion of these next-generation CAR constructs is beyond the scope of this review, also because almost all development took place in the CAR-T field. The only published CAR-NK clinical trial so far employed a second-generation CAR-NK construct that was enhanced with IL-15 expression and inducible Caspase 9 [30]. CAR-T cells that include cytokines enhancing persistence and anti-tumor activity are popularly referred to as 4th generation CARs (Figure 2A) or TRUCKs: T cells redirected for antigen-unrestricted cytokine-initiated killing. Two recent examples of CAR-NK enhancements show that the CAR-NK field follows these developments, adapting T cell-specific signals to NK cells where needed. A first example of the translation of a CAR-T cell enhancements to CAR-NK cells was recently provided by Wang and colleagues [69]. They developed CAR-NK cells with a protein switch that enhances NK cell proliferation and survival, while at the same time coupling CAR 
expression with ectopic IL-15 expression and an inducible suicide gene [69]. In recently published other study, the expression of a CAR/IL-15 construct ("4th generation CAR") is coupled with CRISPR/Cas9-mediated knockout of CIS, a negative regulator of IL-15 signaling [70]. This interesting strategy boosted CAR-NK cell function in vitro and in xenograft models, at least in part through increased aerobic glycolysis. This double enhancement of IL-15 signaling is likely to be beneficial in the tumor microenvironment that is often limited in IL-15 levels [70]. Modification of cytokine signaling in CAR-NK cells may thus provide further possibilities for CAR-NK cell improvement. For instance, cytokines like IL-12, IL-15, IL-18 are responsible for the induction of memory-like NK activity following CMV infections [71,72] or even anti-cancer responses [73]. Future studies will provide more insight in the what other enhancement strategies work best for CAR-NK cells, helping them to overcome challenges posed by the tumor microenvironment (also see Section 8.3).

Most current CAR configurations depend on the CD3 $\zeta$ chain-signaling domain, yielding 3 immuno-receptor tyrosine-based activation motifs (ITAMs) per CAR. In turn, these ITAMs will recruit and activate the Syk or ZAP70 tyrosine kinases, or induce PI3kinase signaling [22]. As CAR-NK cells are usually also designed around the CD3 domain, and again specific studies regarding activation signals in CAR-NK cells are currently lacking, we must rely on findings from the CAR-T field regarding the molecular makeup of downstream activation.

Strong activation signals are important to induce a potent anti-tumor response, but can also lead to quick exhaustion of the effector cells. Therefore, the combination of costimulatory domains can be used to calibrate the desired immune cell response. Compared to 4-1BB-based CARs, CD28-based CARs exhibit an effector profile that is faster and provokes larger-magnitude changes in lymphocyte-specific protein tyrosine kinase (Lck) phosphorylation in the signaling pathway [74]. In this way, CD28-triggered signals induce higher levels of interferon- $\gamma$ (IFN- $\gamma$ ), granzyme $B$, tumor necrosis factor $\alpha$ (TNF-a) [74]. However, it is known from CD28-based CAR-T cells that this strong costimulatory signal also causes activation-induced cell death (AICD), and that the weaker CD3 $\zeta$ ITAM signals leads to better CAR-T cell function and longer persistence $[75,76]$. On the other hand, 4-1BB-CD3 $\zeta$ signals preferentially induce memory-associated genes and sustained antitumor activity [74]. The reason may be that antigen-independent tonic signaling through CD28 domains increases T cell exhaustion while the presence of the 4-1BB domain ameliorate this [77]. The implications of tonic signaling through CAR structures was covered in an excellent review by Ajina and Maher [78].

Moreover, activation signals may have an impact on the metabolism in immune cells. CD28-CAR cells are predominantly dependent on glycolytic metabolism, while 4-1BBcontaining CAR cells exhibited superior persistence as a result from increased oxidative metabolism [79]. It was recently found that calibration of the CD3 ITAM region importantly changes the functional phenotype of CAR-T: introduction of mutations in different parts of the CD3 ITAM motifs leads to a naïve-like T cell phenotype, with high proliferative capacity and longer persistence [76]. On the other hand, NK cells may be induced into cells with a memory-like phenotype after activation with IL-12, IL-15 and IL18 [80]. When an anti-CD19 CAR was introduced in NK cells activated a memory phenotype, these cells showed enhanced in vitro and in vivo anti-tumor activity [80].

Another study shows that there is a lot of room for improvement of the activation signals in CAR cells. In CAR-equipped T cells, proximal signaling downstream of the antigen receptor was significantly reduced compared to normal $T$ cells, due to inefficient recruitment of ZAP-70, resulting in important deficiencies in downstream signaling [81]. 
Thus, the choice of activation signal will have great impact on the functional and persistence of CAR-bearing cells. As these studies were conducted in T cells, there is an important need for structured analysis of the optimal combination of activation domains for NK cells.

We found 72 publications describing CAR-NK cell lines using 90 constructs and 33 studies with 43 different constructs using primary CAR-NK cells (Figure 2E). Both in CAR-NK cell line and primary CAR-NK cell studies, CD3 $\zeta$ is almost universally used as the main activation domain of which about half carry one additional domain, usually adding 4-1BB or CD28. As for third generation constructs, combination of CD28/4$1 \mathrm{BB} / \mathrm{CD} 3 \zeta$ is most often used. These constructs thus provide a means for NK cells to directly receive co-stimulation signals once the CAR binds the tumor antigens. Given the disparities in both antigen sensitivity and number of ITAMs, it is plausible that the smaller number of ITAMs per CAR receptor could result in a comparatively longer persistence of CAR-T cells [76].

To our knowledge, the only direct comparison of different activation signals in CARNK was performed by Li et al [28]. In that study, among 9 different constructs, a 2B4CD3 $\zeta$ based CAR-NK construct showed the most robust CD107a degranulation and antigen specific cytotoxicity. Moreover, a point mutation of Arg-to-Ala in the transmembrane or Tyr-to-Phe in the ITAM/ITSM of NKG2D-2B4-CD3ろ construct sections reduces phosphate recruitment of downstream activation molecules like pSyk, pPLC- $\gamma 2$ and $p E R K 1 / 2$. This will decrease degranulation, cytokine release and antigenspecific lysis capacities of the CAR-NK cells. This indicates that the transmembrane and intracellular domains are also crucial parts that determine CAR-NK cell functionality.

In addition, the combination CD28-CD3 $\zeta$ was also shown to provide strong activation signals and is able to support CAR-NK cell survival for one year in vivo in the first published clinical trial with CAR-NK cells [30]. In general, the CD3 $\zeta$ domain is most often used (Figure 2E), but it still remains to be determined which is the best combination of domains, in which order and in which situation.

\subsection{Detection tags (GFP, cMyc-tag, FLAG, LNGFR)}

Introduction of molecular tags and fluorescent proteins together with CAR genes allows for easy approaches to enrich, quantify and trace the CAR-NK. For instance, various groups use c-Myc or Flag tags directly situated before or after the scFv, which are then expressed together with the CAR on NK cells [82]. Fluorescent proteins are also commonly used in CAR-NK plasmids using bi-cistronic elements, and allow researchers to conveniently trace the expression level of CAR in NK cells [83]. The disadvantage of this latter system is that the detection of the tag does not always reflect the CAR expression (our own unpublished data). A second issue in this case is that fluorescent proteins and other big epitopes are foreign proteins to the human body, and could thus induce an immune response directed to this exogenous epitope. Therefore, these tags are generally used in the laboratory setting, and are removed when clinical studies are started.

\subsection{Synthetic biology: codon optimization and scFv humanization}

Codon optimization is a technique used to alter the use of nucleotides without changing the amino acid sequence. For CAR-NK design, many researchers rely on the use of DNA synthesis for some of the building blocks for their plasmids. Because of technical limitations during synthesis, it may be necessary to change the DNA sequence 
to allow for successful production. Indeed, this codon optimization was mentioned in 8 out of 72 CAR-NK cell line and 5 out of 33 primary CAR-NK reports. It is important to realize that the use of a certain codon set may improve CAR-NK expression in mammalian cells, but may have negative effects on virus production [84]. In one direct comparison of CAR-T cells with and without codon optimization, no differences were found in CAR surface expression, tumor eradication and cytokine production [85].

A
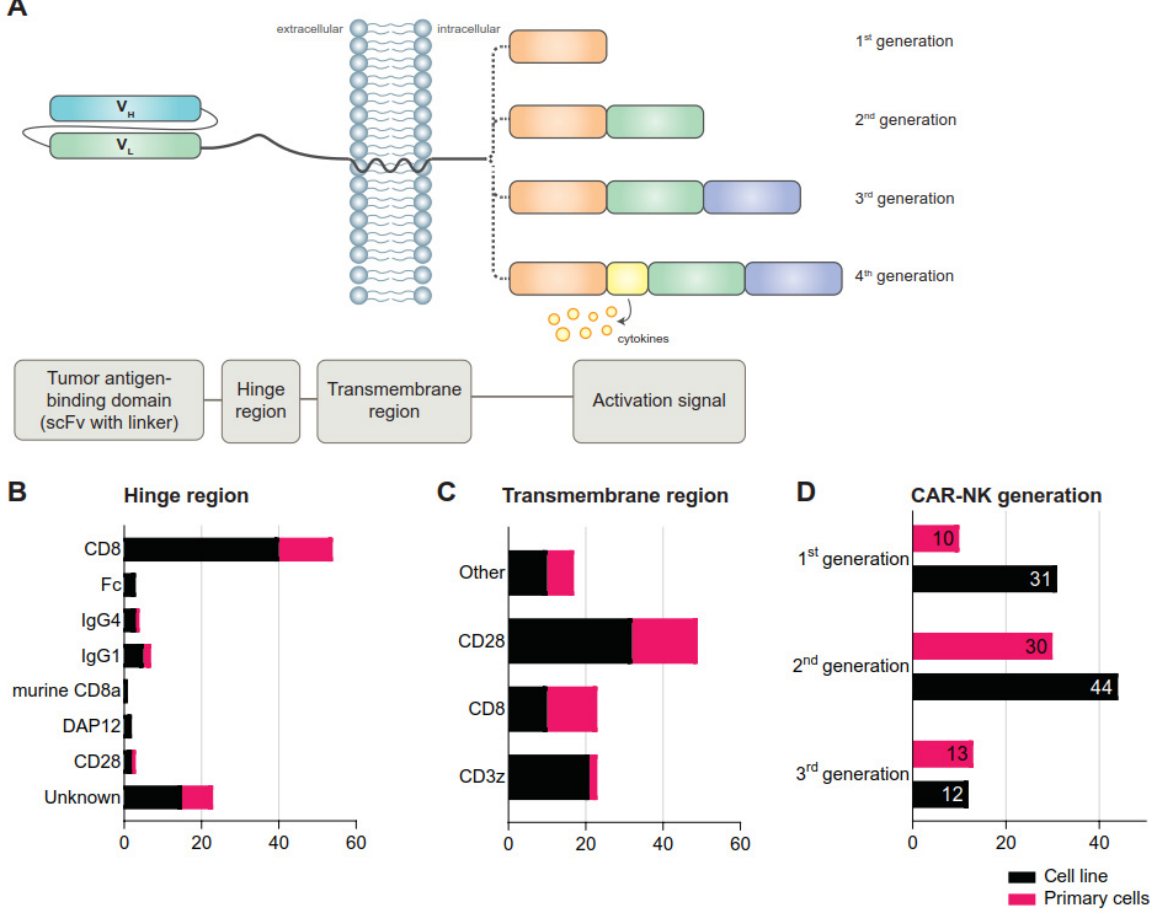

E
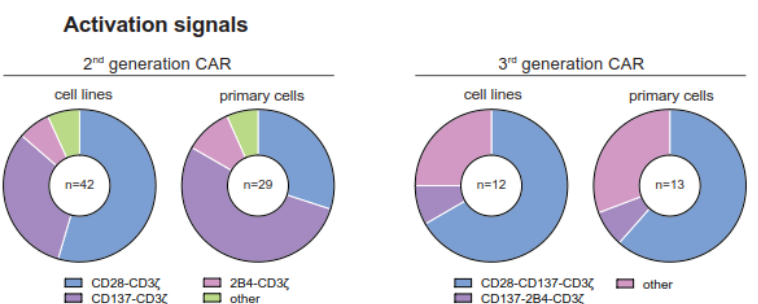

Figure 2. CAR-NK cell design.

A) Schematic overview of the building blocks for CAR-NK cells. From left to right: the extracellular single chain variable fragment (scFv) consists linker-bound heavy and light chain and it determines the specificity of the CAR. The hinge region connects the tumor antigen-binding domain to the transmembrane region, ensures CAR stability and provides the flexibility for easy access to the target protein. The transmembrane region joins the extracellular and intracellular CAR domains, docks the CAR to the cell membrane and transduces activation signals to the intracellular activation signals. The number of intracellular activation domains determines the CAR generation. Various combinations of activating domains are used to mount a strong antitumor response. One example of a " $4^{\text {th }}$ generation" CAR is shown, co-expressing stimulating cytokines. Other examples of next-generation CAR constructs are discussed in Table 3. 
B and C) Analysis of the most frequently used hinge (B) and transmembrane (C) regions for CAR-NK construction. Only studies reporting on the hinge region were included for this analysis.

D) Quantification of experimental studies with the indicated CAR-NK generations for cell line-derived (black) and primary NK cell-derived (pink) CAR-NK cells.

E) Pie charts showing the activation signals used for cell line-derived and primary cell CAR-NK cells, separated by CAR generation. Only the most frequently used combinations are shown.

The starting point for the development of new CARs often are murine antibodies recognizing a tumor-associated antigen. However, murine antibodies cannot directly be used for clinical use, as the non-human regions of the mouse antibody are often immunogenic. Therefore, these regions can be replaced with corresponding human sequences in a process called "humanization". Indeed, this technique is also successfully applied for the development of CAR scFv without negative effects on CARNK killing capacity $[58,86]$.

\section{Transfection or transduction vehicle for CAR}

\section{expression}

With the progress in gene modification technologies, numerous approaches have been applied to generate CAR-NK. The two main methods are viral transduction (using lenti- or retroviruses), or transfection with either naked plasmid DNA, transposase DNAmediated integration or mRNA by electroporation [14] (Table 4). 64 preclinical studies describing CAR-NK cell lines using viral transduction and 11 studies applying electroporation or nanoparticle-mediated transfection have been published to date. In primary CAR-NK cell studies, 29 described viral transduction and 9 publications conducted electroporation as method of choice (Supplemental Table 1, Supplemental Table 2 and Figure 3A). Monoclonal or polyclonal NK cell lines can be generated using FACS or (Clini-) MACS, but this creates a more complex Good Manufacturing Practice (GMP)-compliant production process. An important difference between the various technologies is the duration of stable CAR expression. For longer expression (multiple weeks) in primary cells, viral transduction is usually employed, while mRNA electroporation results in transient expression lasting for about one week. CAR expression levels in primary NK cells strongly vary (20-70\%) and are thus sometimes low, but most studies still report highly specific and effective killing of target-positive tumor cells.

\subsection{Lentiviruses}

Lentiviruses have been widely used in the gene therapy space for decades, as they are able to transduce cycling and non-cycling cells with high transduction efficiency. They have been successfully applied in curing people from diseases including HIV and cancer [87]. Lentiviruses have the advantages that they integrate into the host genome, a feature that can lead to permanent expression of the transgene, and low intrinsic immunogenicity [88]. To date, there are 14 reports on primary CAR-NK cells and 44 papers on CAR-NK cell lines that have successfully used a lentivirus as vehicle (Figure 3A). Among pre-clinical studies, 21 studies used a 2nd generation virus and 6 studies used a 3rd generation lentivirus to generate a CAR-expressing NK cell line (17 unknown) (Figure 3B). In the primary CAR-NK cell studies, 5 studies used 3rd generation lentivirus 
while 7 reports describe 2 nd generation lentiviral vectors (2 unknown) (Figure 3B). The 3 rd generation lentiviral vectors are being considered safer than the 2 nd generation due to gag/pol and rev viral genes that are located on separate plasmids, thus making wildtype recombinant virus generation even more unlikely [89]. In order to improve the fraction of CAR-expressing cells after transduction, CAR-NK cells can be enriched using Fluorescence Activated Cell Sorting (FACS) or through antibiotic selection. Most studies applied a third generation lentivirus under GMP conditions for clinical application and often showed a transduction efficiency lower than $10 \%$ on NK cells [90], explained by the low binding capacity of the VSV-G envelope to the LDL-R, which is the receptor facilitating entry of the virus. Changing the virus pseudotype (e.g. to BaEV) was suggested to enhance the viral transduction efficiency as it targets other receptors expressed at higher levels in NK cells [91,92]. Furthermore, stimulation with cytokines or certain compounds can facilitate higher transduction rates in NK cells [92-94] (discussed further in Chapter 5). All combined, these results have demonstrated that lentiviruses are a powerful vehicle to manufacture CAR-NK cells for clinical disease.

\subsection{Retroviruses}

Retroviruses have been used as gene therapy vectors for decades [95]. Retrovirusbased vectors require NK cells to be actively dividing to obtain integration of the vector into the genome [96]. There are 19 studies using CAR-NK cell lines and 16 reports using primary NK cells published that employ retroviruses (Figure 3A). One recent Phase I clinical trial targeted CD19+ non-Hodgkin's lymphoma and chronic lymphocytic leukemia by infusion of retrovirus-transduced anti-CD19 CAR cord blood NK cells. In this study, $73 \%$ of patients responded with 7 out 8 patients attaining complete remission. Moreover, responses were fast and seen within 30 days after CAR-NK infusion at all dose levels. After one-year follow-up, expanded CAR-NK cells could still be detected by qPCR [30]. After infusion, CAR-NK DNA copy numbers remained stable for up to one year in peripheral blood and measured between 102 to 104 copies of the CAR-NK vectors per $\mu \mathrm{g}$ genomic DNA. These results show for the first time that retrovirally transduced CARNK cells can persist in vivo for a long time.

Different genera of retroviruses have been used to generate CAR-NK cells (Figure 3B). Alpha retroviruses carrying the RD114 envelope are superior in transduction efficiency of primary NK cells compared to gamma retrovirus and lentivirus [97]. Recently, Muller et al. showed that RD114 alpha-retroviruses result in 3 times higher CAR-NK transduction efficiencies (around 45\%) than VSV-G lentiviruses on day 3 in primary NK cells (about 15\%) [91]. However, from day 7 onwards, retroviruses and lentiviruses showed equal performance [91]. Retrovirally transduced CAR-NK cells can be expanded with sustained CAR expression for at least 2 weeks. Although long and stable CAR expression in NK cell can be achieved using different retroviruses, safety of the retrovirus system is still a concern, especially when compared to safer lentiviruses.

\subsection{Electroporation of mRNA}

Electroporation of CAR encoding mRNA is a swift and efficient but transient approach to generate CAR-NK cells. Some companies already developed equipment to facilitate electroporation in a closed system compatible with CAR-NK cell generation, which is especially interesting for GMP-compliant production starting from primary NK cells. There are 11 studies in CAR-NK cell lines and 10 primary CAR-NK cells that rely on electroporation (Figure 3A). The electroporated cargo mainly consists of CAR-encoding 
mRNA or plasmids (Figure 3B). With the current technological advances and the use of high-purity CAR mRNA instead of cDNA in a plasmid, transfection efficiencies in NK cells have increased dramatically, achieving up to $95 \%$ with minimal negative effects on cell viability $[98,99]$. Generally speaking, mRNA transfection efficiencies are much higher in expanded or activated NK cells (more than 60\%) than in freshly isolated NK cells (about $40 \%$ ) [100]. As mRNA synthesis is compatible with GMP regulations, and electroporation can be done in a clean room, it is thus feasible to generate GMP-compliant CAR-NK via mRNA electroporation. However, the main disadvantage of this method is the narrow, transient window of CAR expression: after electroporation, the CAR-NK cells should be transfused back into patients within 7 days.

\subsection{Sleeping Beauty transposon}

Transposon-based systems can introduce CAR transgenes with higher efficiency and at predefined locations, which is an important advantage over conventional methods that do not possess an integrating element. Transposons are mainly introduced into NK cells by electroporation followed by integration into the host genome through transposase enzymes [101,102]. Two studies applied the transposon system to generate CAR-NK cells: one used NK-92-MI cells [101], the other study described transposon transfection into iPSC cells followed by differentiation into NK cells [28] (Figure 3A). Both studies used the 4D-Nucleofector electroporator to introduce the transposase encoding plasmids into cell nucleus (Figure 3B). After enrichment, anti-mesothelin-CARs were stably expressed on iPSC-derived NK cells and were functional in a murine model of ovarian cancer [28]. Although there are many studies using the transposon system to generate primary CAR-T cells [53,103], primary NK cells are much more difficult to transduce using transposons. We expect that with further advances in transposon and transfection methods, generation of CAR-NK cells using transposon will become a more viable approach.

\subsection{CRISPR/Cas9-mediated strategies}

CRISPR/Cas9 has recently emerged as a powerful technique for genetic modification. This technology relies on the introduction of Cas 9 protein in conjunction with guide RNA into the NK cells. Initially, this technique was used in primary NK cells to disrupt the CD38 gene, aiming to prevent fratricide of NK cells as CD38 is expressed both on NK cells and multiple myeloma and AML cells [104]. More recently, CRISPR/Cas9 has also been applied to introduce new genes [105]. Here, in parallel to CRISPR/Cas9, a homologous donor DNA template is introduced in the same cell via transfection [106]. This DNA template replaces the targeted gene, thus allowing for the introduction of genes that promote the anti-tumor effects. This technique was first successfully applied in primary T cells: CRISPR/Cas9 was used to target the TCR alpha gene, relying on homology-directed repair (HDR) to knock-in a CD19-CAR cassette. The expression of the CD19 CAR, now under the control of the endogenous TCR alpha promoter, manifested much stronger expression that also persisted longer compared to lentiviral vectors using a viral promoter $[40,107]$. Following this paradigm, one can apply this concept to generate more potent and persistent CAR-NK cells. In several studies that employ HDR templates, knock-in efficiencies of more than $75 \%$ were obtained using K562-mIL-21-expanded NK cells (see also paragraph 5.4) [43]. 
A

\section{CAR-NK transduction}

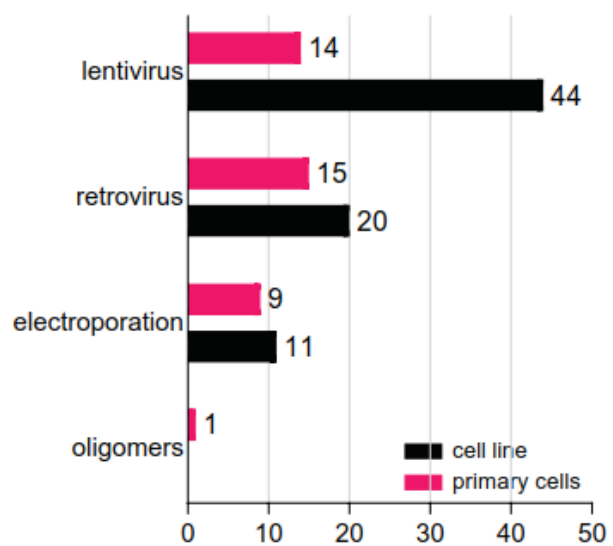

C

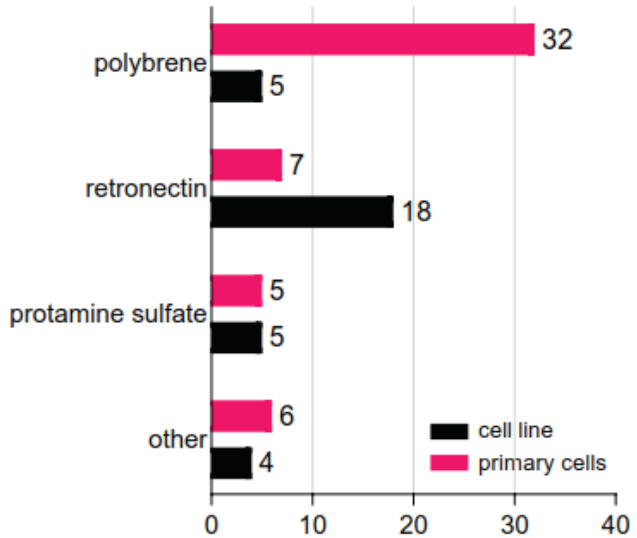

B Transduction vectors

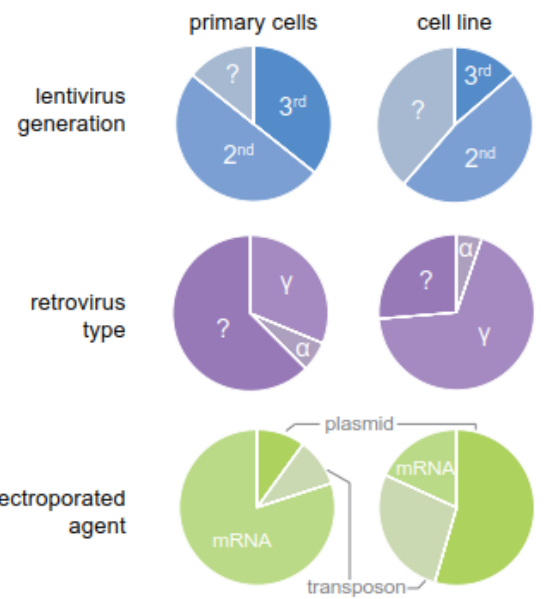

D Primary NK cell source

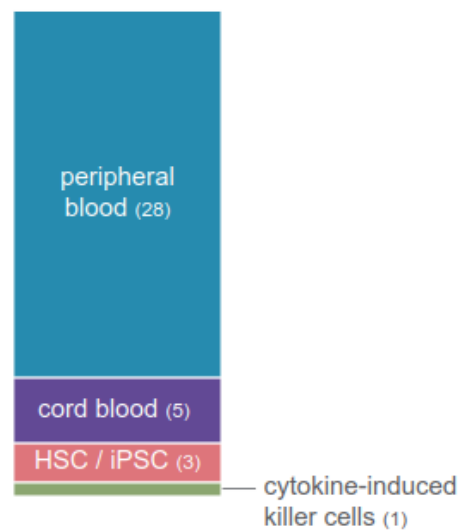

Figure 3. Optimization strategies for CAR-NK cell generation.

A) Different vectors can be used to transduce NK cell lines and primary NK cells with CAR constructs. Quantification of the vectors used for CAR-NK generation.

B) Details of the various vectors used for CAR-NK generation. In blue, lentivirus generation, with $3^{\text {rd }}$ generation viruses considered the safest option with minimal risk of in vivo recombination with other lentiviruses. In purple, the genus of retrovirus (alpha or gamma, or unknown). In green, the electroporated vector.

C) Various compounds have been identified that can be used to enhance virus-mediated transduction. For studies that employ virus-based strategies for CAR-NK generation, the methods used for enhancing virus transduction were quantified.

D) Primary cells for generation of CAR-NK cells are derived from different sources. Quantification of the four sources for primary NK cells for CAR-NK generation.

However, in fresh NK cells, the Cas9 knock-in efficiency is only 3\% 16\% [42]. When the CAR gene is delivered via viral vectors or using transposon-based random insertion, expression levels are variable. CAR expression driven by an endogenous promotor 
following CRISPR/Cas9-specific locus knock-in strategies could result in long CAR-T persistence in vivo, with a memory-like phenotype and less expression of exhaustion markers [40]. Thus, the CRISPR/Cas9 strategy is a versatile technique that should be further explored for the generation of CAR-NK cells. It can be used to precisely delete, repair or introduce genes in a specific locus, hence holding promise to generate powerful anti-tumor NK cells [43].

\section{CAR-expression detection methods}

Following transduction of the chimeric antigen receptor, various techniques are available to assess CAR expression levels on the cell surface and to determine the efficacy of the developed CAR-NK cells. The most employed experimental modalities include flow cytometry, (fluorescence) microscopy and analysis of gene expression levels. In addition, flow cytometry and gene copy number analysis are used to monitor CAR-NK cells in patients. Here, we discuss the advantages and disadvantages of the various approaches.

\subsection{Flow cytometry}

Flow cytometry is widely used to detect CAR expression, since it is a fast and reliable detection method that provides quantitative data on protein expression at the single cell level. This technique relies on the use of fluorescently labeled antibodies that recognize parts of the CAR complex on the membrane. In addition, flow cytometry can be used to detect fluorescent tags or tags that were specifically added to the transduction cassette to facilitate expression recognition as was introduced in section 2.8.

Labeled antibodies or their derivate, such as antigen-binding fragments (Fab), can be used to detect components of the CAR complex on the cell membrane. Commonly, these antibodies target the antigen-recognizing ScFv part of the CAR. In addition, the CAR ligand with a fragment crystallizable region $(\mathrm{Fc})$ tail are used in conjunction with Fc-recognizing labeled antibodies. While these methods have the advantage that they are very specific for the detection of a specific CAR, this is also the main drawback of this approach: some antibodies are generally not commercially available [108].

To overcome this challenge, universal detection methods have also been developed. For instance, protein $\mathrm{L}$, a bacterial-derived product that recognizes the variable part of the light chain, binds to most immunoglobulin classes, including scFv that are used in CAR development [109]. Protein $L$ has successfully been used to detect the expression of murine and human CAR on peripheral blood cells, including NK cells [110].

Another strategy comprises the incorporation of tags specifically included to facilitate the detection of the CAR. For this, molecular tags such as poly-histidine-tag ("His-Tag"), FLAG, or c-Myc tags have been incorporated into the CAR construct to facilitate easy detection using commercially available monoclonal antibodies [111]. Even though these tags are small and considered lowly immunogenic [112], it is undesirable to include nonessential and foreign sequences in products designed for clinical use.

Indeed, in the experimental setting other detection methods can also be used to help in CAR detection. For instance, fluorescent tags (e.g. GFP) or non-native receptor proteins, such as LNGFR [113], can be added to the CAR construct. While these methods simplify CAR detection, it is important to realize that fluorescent proteins or additional receptors are included in the CAR construct using bi-cistronic elements, or 
their expression is driven by additional promotors. Thus, the expression of the tags may not reflect CAR expression, and may only be a marker of successful transduction.

\subsection{Quantitative PCR}

Quantitative PCR is a well-established technique for quantification of CAR transgene expression levels. As the technique requires disruption of individual cells to isolate genomic material, this technique does not provide direct information about CAR expression at the single cell level. However, surrogate markers that are expressed at relatively stable levels (housekeeping genes) can be used to estimate the number of cells and to follow copy numbers over time. Another limitation of PCR-based techniques is that they do not provide information on viable effector cells, nor do they take into account that not all transferred cells will be bone fide CAR-expressing effector cells.

Given these limitations, qRT-PCR is mostly used for follow-up studies in clinical trials in which flow cytometry cannot be used due to the low frequency of circulating CARpositive effector cells. As an example, in a recently published phase I/II trial using CARNK to target CD19-expressing malignancies, gene copy number analysis was used to measure in vivo expansion of CAR-NK [30]. The viral vector transgene copy numbers were normalized to the amount of genomic DNA. Using this technique, the authors were able to show expansion of CAR-NK cells as early as 3 days after infusion, and demonstrated that CAR-NK cells persisted in patients for at least 12 months.

Novel techniques, such as droplet digital PCR (ddPCR) allow for absolute quantification of single molecules using an advanced microfluidics system [114,115]. The main advantage of this technique is that it eliminated the need for a standard curve, and therefore allows for reproducible analysis of very low copy numbers. This is likely to be important of the CAR-NK field, as the number of CAR-NK cells in a patient is relatively low, especially at later time points. However, at this moment, ddPCR has important disadvantages: sample handling is much more complicated, time-consuming, expensive and relies on small-size chips or cartridges, disqualifying the technique for large numbers of samples.

\subsection{Western Blot}

Western blots that employ antibodies recognizing an antibody, as for flow cytometry, can also be used to detect CAR expression in a cell population [111]. Immunoblots do not provide information at the single cell level, are time-consuming, and therefore are not routinely used to confirm CAR incorporation. However, they can provide valuable information about the size of the complex in which CAR is incorporated and are thus often used in CAR optimization studies. For instance, several studies have shown that CARs need to form dimers to be responsive, and that they need to engage in a complex with signaling domains to be effective [116].

\subsection{Confocal microscopy}

Fluorescent and electron microscopy can be used to visualize CAR molecules on the surface of effector cells. Similar to flow cytometry and western blot, this technique relies on the use of antibodies that can be CAR-specific, or universal antibodies that recognize common CAR components. Indeed, fluorescent and electron microscopy have been used to detect CAR molecules on the surface of transduced NK cells [117]. Given that microscopic analysis is low-throughput, these techniques may not routinely be used to confirm CAR expression, but can be valuable tools to visualize the correct incorporation 
of the CAR in the plasma membrane and to determine the number of CAR molecules per cell.

\subsection{Molecular imaging}

In addition to follow-up by flow cytometry and determination of gene copy numbers, various molecular imaging strategies are currently under investigation for experimental and clinical use. Following transduction, cells are loaded with tags that can be detected using optical, nuclear or magnetic resonance techniques [118]. While fluorescent and bioluminescence techniques are only viable for use with laboratory animals, positron or photon-emission tomography (PET/SPECT) and magnetic resonance imaging can also be used in humans. These techniques can be used to gain insight in the NK cell biology at the cellular and molecular level, as they allow for quantitative tracking of (CAR-) NK cell biology in vivo [118]. However, most of these techniques are still in the very early phases, requiring complicated and expensive labeling procedures and long acquisition times.

\section{Approaches for enhancing NK cell virus-mediated}

\section{transduction}

Lentiviruses and retroviruses are the most commonly used systems to induce stable expression of chimeric antigen receptors in NK cells. Encouraging results have been shown for the transduction of NK cell lines and primary NK cells, including their use in clinical trials $[89,119]$. However, attaining high viral transduction efficiency while maintaining good cell viability remains a challenge, especially for primary NK cells. Various strategies have been developed to enhance viral transduction of NK cells (Figure 3C).

These approaches often target the entry of a vector into NK cells, either via changing electric charges of cells or by targeting the viral envelope or the receptor on the target cell $[120,121]$. Therefore, changes to the envelope on the viral surface and up-regulation of receptors on the target cell are the main approaches to boost transduction $[89,120]$.

\subsection{Enhancing NK cell transduction via upregulation of LDLR using statins}

Most lentiviruses used for gene transductions are pseudotyped with a VSV-G envelope glycoprotein. The main receptors for this envelope protein are the low-density lipoprotein receptor (LDLR) and phosphatidylserine [122]. NK cell lines and primary NK cells express low levels of LDLR. Interestingly, statins that are used in the clinic to lower blood cholesterol levels, were shown to induce LDLR on B and T lymphocytes [123]. Similarly, we found that statins also induced LDLR levels in NK-92 and primary NK cells [93]. Consequently, transduction efficiency increased after treatment with statins. However, statins have also been reported to suppress the cytotoxic capacity of NK cells, which can be completely reversed by geranylgeranyl-pyrophosphate (GGPP). Not all statins are potent boosters of viral transduction: rosuvastatin in combination with GGPP most potently improved viral transduction without affecting the cytotoxic properties of the NK cells [93]. 


\subsection{Other small molecular compounds to enhance NK cell transduction}

In addition to statins, other compounds have been tested to enhance the viral transduction of NK cells. Negative charges existing on both the viral envelope glycoproteins and the target cell receptors can be detrimental for transduction [124]. Therefore, cationic polymers, such as protamine sulfate, polybrene [125] and dextran [126] can be used during transduction to positively charge the cell surface [124] (Figure 3C). Using high-throughput analyses, other compounds that enhance lentiviral transduction have also been identified. Phorbol 12-myristate 13-acetate (PMA) [127], rapamycin [128], Cyclosporin A [129], P338 poloxamer [130], prostaglandin E2 (PGE2) [131], Vectofusin-1 [132] and RetroNectin [92] have all been reported to promote lentiviral transduction on hematopoietic stem cell (HSC), T cells or B cells. However, in our analysis on the effects of these compounds on NK cell transduction, we found that only statins consistently promote viral transduction without major negative effects on cell viability [93].

Next to compounds that promote viral transduction, substances that inhibit antiviral responses in NK cells can also be used to enhance transduction. Indeed, BX795, an inhibitor of the TBK1/IKKE complex that controls antiviral responses in NK cells, was demonstrated to promote lentiviral gene transduction efficiency by 3.8-fold [133].

\subsection{Alternative lentiviral pseudotypes for NK cell transduction}

VSV-G has long been used as the major glycoprotein for pseudotyping lentiviruses because of its broad tropism, facilitating the transduction of a wide range of cell types [134]. VSV-G lentiviruses have also been used to generate genetically modified NK cells for decades, and make stable NK cells line $[135,136]$. However, as alluded to above, the low expression levels of the VSV-G receptor LDLR on fresh human NK cell hinders efficient and easy lentiviral transduction of NK cells [93,94]. As an alternative to boosting LDLR expression levels, other glycoproteins can be used to pseudotype the viral particle [121].

Colamartino et al. showed that BaEV lentiviruses outperform VSVG-, MV- and RD114-pseudotyped viruses in human NK cells, even using activated NK cells [92]. Their study supports the idea that the abundance of the lentivirus receptor on the target NK cell may correlate with the efficiency of the integration of the gene of interest. The receptors for BaEV are ASCT1 and ASCT2, which are highly expressed on activated NK cells, both at the mRNA and protein level [92,94]. Therefore, BaEV lentiviruses are potentially superior to VSV-G lentiviruses for gene therapy in NK cells [92].

RD114 pseudotyped retroviruses have also been used to generate human NK cells expressing an anti-CD19-CAR [137]. Suerth et al. demonstrated that efficient modification of human NK cells by using an alpha-retroviral vector with a RD114/TR envelope [97]. VSV-G-pseudotyped lentiviral particles normally outperform VSV-G pseudotyped alpha-retroviral and gamma-retroviral particles. However, when alpharetroviral particles were pseudotyped with RD114/TR, this resulted in superior transduction performance. These results show that the compatibility of virus particles and the surface glycoprotein will ultimately determine the efficiency of viral transduction [97]. With these concepts, further studies towards modifying the component of the viral glycoprotein specific for NK cells may improve the LVs transduction efficiency [121]. 


\subsection{Cytokines and K562-m/L-21/4-1BBL feeder cells}

The cytokines and feeder cell lines that are used to expand NK (see section 7) cells may also have positive effects on transduction with retro- and lentiviruses. Previous studies demonstrated that IL-2 induced upregulation of the viral receptor LDLR in NK cells, resulting in enhanced transduction, proliferation and cytotoxicity [138]. The addition of soluble IL-2, IL-12 and IL-21 to the NK cell medium have also been reported to enhance VSV-G lentiviral transduction, with a possible synergistic effect of additional PHA $[133,135]$. Other reports have indicated that culturing primary human NK cells with K562 feeder cells with membrane-bound IL-21 and 4-1BBL also boosts lentiviral and retroviral transduction of NK cells $[92,139]$. Thus, these methods are already used and could be easily implemented to further enhance viral transduction.

\subsection{Microfluidic mechanic devices}

The titer of the virus is a crucial parameter of the viral transduction. Although with the advancement of viral vectors and transfection technologies, it remains difficult to fully standardize the method to obtain a high enough and consistent titer to perform a successful transduction. Even though this system has not been tested for NK cell transduction, the microfluidic platform systems developed by Luni et al. [140] and Tran et al. [141] may develop into a new versatile tool to improve the transduction efficiency while working at low viral titers in a sequential manner.

\section{Cell sources for CAR-NK generation}

NK cell-based immunotherapy requires relatively large numbers of effector cells. The life span and proliferative capacity of NK cells is limited, and extensive ex vivo handling of NK cells might influence their cytotoxic capacity. Multiple sources have been used to isolate and generate sufficient NK cells for CAR-NK applications (Figure 3D and Table 5). In this section, we will discuss the advantages and disadvantages of these NK cell sources for the development of CAR-NK cells.

\subsection{Peripheral Blood NK cells (PB-NK)}

NK cells can be isolated relatively easily from the patient itself (autologous PB-NK) or from healthy donors (allogeneic PB-NK), and therefore most (31/33) preclinical CARNK studies use PB-NK. The majority of these studies use allogeneic NK cells from healthy donors (Figure 3D). For cancer immunotherapy, autologous NK cells are typically not very effective, as they are functionally silenced when they encounter self$\mathrm{MHC}$ antigens. Furthermore, the function of patient-derived NK cells is often compromised by the underlying disease or previous treatment. Therefore, allogeneic PBNK are often preferred for immunotherapy purposes, even though this requires careful depletion of the T lymphocytes from the product, as these latter cells could induce graftversus-host reactions [142]. Since NK cell numbers in peripheral blood are relatively low, NK cells are routinely expanded after isolation. Even though the number of CAR-NK cells required for a meaningful response has not been established, previous NK cell-based therapies typically infused $10^{5}$ to $10^{8} \mathrm{cells} / \mathrm{kg}$, thus requiring as many as $10^{10} \mathrm{NK}$ cells for a patient weighing $100 \mathrm{~kg}$. Therefore, various expansion protocols have been developed, which will be discussed further in this review (section 7). 
The main advantage of PB-NK cell sources for cancer immunotherapy is that the cells are already mature, and do not need to undergo a lengthy differentiation protocol, as for stem cell-derived sources. On the other hand, the transduction efficiency of PB-NK is relatively low (discussed in sections 3 and 5), and prolonged culture often leads to telomerase shortening and reduced cytotoxicity due to exhaustion, even though these problems can be largely circumvented by using the correct combination of stimulatory molecules [143-145].

\subsection{Cord blood-derived NK cells (CB-NK)}

An important limitation of PB-NK cells is the dependency on the availability of healthy donors at the time the NK cell expansion process needs to be started. In a clinical setting where the new diagnosis of cancer warranting CAR-NK treatment cannot be planned, this may pose a problem in logistics and planning. Therefore, alternatives starting from frozen material, becoming off-the-shelf products, are an attractive alternative. However, it has been reported that freezing of mature PB-NK cells importantly decreases their viability and cytotoxic capacity $[146,147]$. In general, large numbers of NK cells can be obtained from umbilical cord blood samples, due to the high proliferative capacity of these cells. For instance, only $10 \%$ of one cord blood unit is required to generate an almost pure pool of more than $10^{9} \mathrm{NK}$ cells in two weeks [117], which is usually sufficient for one treatment cycle. Another advantage of CB-NK cells is that the haplotype of the sample can be determined at the time of collection, allowing the generation of a cell bank from which HLA-mismatched NK cells can be selected on demand.

There are also some concerns about the cytotoxic capabilities of CB-NK cells. The expression of natural cytotoxicity receptors is lower in CB-NK cells that were expanded in an artificial antigen presenting cell system compared to IL-2-expanded cells. However, the expression levels of KIRs, NKG2A, CD94 and NKG2C was similar and no differences in cytotoxicity in killing multiple myeloma cells was observed [148].

Although only a minority of the pre-clinical CAR-NK studies uses cord blood as a cell source (Supplemental Table 2), the potency of CB-NK was recently confirmed in the first publication of a clinical trial using CAR-NK cells [30]. Here, CB-NK cells were expanded on K562-mbIL21 and 4-1BB ligand feeder cells and endowed with a CD19directed CAR, ectopic IL-15 production and an inducible suicide gene. Remarkably, qPCR showed that these CB-CAR-NK cells persisted more than 270 days in vivo [30]. It is yet to be established if this finding correlates with long-lasting clinical responses as well.

\subsection{Stem cell-derived NK cells: differentiation of NK cells from hESC and iPSC}

Disadvantages of PB-NK and CB-NK as cell sources for CAR-NK development include the natural heterogeneity between donors, resulting in variance in performance of the final NK cell product. For that reason, stem cell-derived NK cell expansion is an attractive alternative to develop a standardized, off-the-shelf therapeutic product.

Indeed, clinical-scale NK cell production starting from human embryonic stem cells (hESC) or induced pluripotent stem cells (iPSC) has been demonstrated [149-151]. The use of commonly available hESC/iPSC cell lines is often preferred over the use of stem cells from bone marrow biopsies, G-CSF mobilization or human embryos [144,145]. Next to ethical and practical issues attached to the use of these sources, the outcome of NK cell production starting from primary stem cells is less predictable and often less efficient 
(reviewed in [152]). The production of NK cells starting from hESC or iPSCs takes 3-5 weeks, and is therefore significantly longer than NK cell expansion starting from peripheral blood- or cord blood-derived NK cells.

When considering the use of hESC-derived NK cells, it is important to note that NK cells derived from the H9 hESC cell line showed limited allogeneic immune response, while they showed a more mature cytotoxic phenotype compared to CB-NK [150]. While we are not aware of any pre-clinical or clinical studies using hESC to generate CAR-NK cells, it is probably technically feasible.

On the other hand, iPSC-derived NK cells that were already shown to be functional against tumors $[151,153]$ have recently been used to generate CAR-NK cells. After optimization of the activation domains for the use in iPSC-derived NK cells, mesothelintargeting iPSC-CAR-NK cells were shown to be as potent as CAR-T cells [28]. Interestingly, tumor-bearing mice treated with CAR-iPSC-NK showed less pathogenic organ damage and lower IFN-y and IL-6 levels than CAR-T-treated in a murine xenograft model.

There are still challenges ahead before iPSC-NK cells can be safely used for the generation of CAR-NK for clinical application. Firstly, CAR-iPSC-NK cells ceased proliferating in vivo after the exogenous administration of cytokines was stopped in the murine model. Systemic administration of cytokines in a clinical setting is highly undesirable, since it is not only costly, but also potentially dangerous. Secondly, iPSCderived cells always bear the potential of malignant transformation. Even though there were no signs of transformation in the present studies, this point may not be neglected during long-term observations. Lastly, iPSC-derived cells are potentially immunogenic, which may lead to the destruction of the effector cells, or even to adverse immune reactions, such as cytokine release storms.

\subsection{NK cell lines: NK-92}

The aforementioned NK cell sources have one major disadvantage in common: obtaining large numbers of NK cells is relatively cumbersome and time-consuming. In general, cell lines circumvent most of these problems, as they are easy to maintain and expand. Therefore, it is not surprising that 72 experimental studies have reported the use of NK cell lines for the development of CAR-NK cells. More than $80 \%$ of these studies use the lymphoma-derived NK-92 cell line [154]. Out of the six NK cell lines available, NK-92 has high anti-tumor activity and is capable of direct cytotoxicity. When NK-92 cells are transfected with CD16, this cell line can also trigger antibody-dependent cell-mediated cytotoxicity (ADCC) [155].

For CAR-NK cell development, NK-92 cells have another main advantage: the cell line also can be easily genetically modified using non-viral methods, including electroporation [156]. So far, NK-92 cells have been tested in pre-clinical CAR-NK studies targeting AML (directed to CD33), lymphoma (CD19), myeloma (CS1), prostate cancer (EpCAM), breast cancer (Her2), neuroblastoma (GD2), glioblastoma (EGFR) and ovarian cancer (mesothelin). Details on these trials can be found in Table 1 and Supplemental Table 1.

One of the major disadvantages of the NK-92 cell line is that the cells are tumorderived and are aneuploid [154]. Therefore, they need to be irradiated before infusion, preventing in vivo proliferation and limiting their lifespan. In phase I trials, NK-92 administration was shown to be safe with only minor reactions $[157,158]$. The number of head-to-head comparisons of CAR-equipped primary NK cells versus NK-92 cells is 
limited. In a study using a CD123-targeting CAR, primary NK cells were shown to be less effective than NK-92 cells in the eradication of AML cells in vitro [159]. This was possibly due to lower surface expression levels of the CAR in primary NK cells. However, it is difficult to draw a concrete conclusion whether the irradiated CAR-NK-92 cells is outperforming the primary CAR-NK cells on overall survival of patients, since the NK-92 cells are replication-deficient. Therefore, their persistence needs further investigation.

With only one CAR-NK clinical trial completed, it is impossible to draw conclusions on the usability of NK cell lines for CAR-NK therapy. Results from the CAR-T field indicate that long-term persistence is important for achieving and attaining complete remission [160]. While NK-92-CAR cells will not sustain in vivo, it is relatively easy to perform multiple infusions of this cell product. Further studies will be necessary to compare how NK-92-CAR can be used in the clinic.

\section{NK cell expansion}

Allogeneic NK cells have been proven safe and efficient to treat patients with AML and ALL $[23,24]$. However, to achieve the number for clinical therapy a large scale GMP expansion of NK cells ex vivo is necessary as the numbers present in peripheral blood of a donor is not sufficient. Several methodologies have been described to facilitate a successful NK cell expansion (Table 6). Expansion can be achieved culturing the cells in various cytokines mixtures (IL-2, IL-12, IL-15, IL-18 and/or IL-21], K562 cell-based cytokine transfected cells (e.g., with IL-15 or IL-21 in combination with 4-1BBL), or autologous PBMC stimulated cells. With the development of automatic and closed expansion equipment, like Miltenyi's Prodigy, Lonza's Wave and G-Rex static bioreactors, it has become feasible to expand NK cells according to GMP requirements.

However, the time it takes to reach clinically relevant numbers may be very long as exemplified by Lapteva et al. who showed that their cytokine mixture needed expansion of NK cells during 4 to 10 weeks [161]. Although this is biologically relevant, for large scale production the time it takes is too long and thus not commercially and practically interesting.

It is also important to note that, NK cells are generally easier to genetically modify after they have been activated by cytokines. While this is an important advantage, it also implies the need for an increased amount of the transduction agent. Obviously, a balance needs to be sought here, which is also clear from the fact that most studies report that viral transduction of NK cells was conducted after 7 days of activation and expansion.

The advancement of NK cell expansion technologies also facilitates CAR-NK cell generation. Co-culture of NK cells with the leukemia cell line K562, often transduced with membrane bound cytokines, recently gained attention as a relatively easy way to obtain large numbers of NK cells. After 7 days of expansion with K562-mIL-21/4-1BB-L feeder cells, the transduction efficiency of expanded NK cells can be above $80 \%$ compared to below $20 \%$ on freshly isolated NK cells [92]. In total, at this moment there are 13 studies published that used the K562 cells as feeder cells for CAR-NK cells to expand, including 12 studies using K562-mIL-15 feeder cells, 3 groups used K562-mIL-21 cells and 1 publication used the parental K562 cell. NK cells expanded with mblL15 showed reduced telomere lengths, while those propagated with mblL21 showed more elongated telomere lengths than freshly isolated peripheral blood NK cells [143]. This suggests that mbIL-21 is an attractive option to expand cells over many generations to reach clinically relevant 
cell numbers. Clinically relevant expansion levels have been reached in 2 weeks [162] (and our own unpublished data).

\subsection{Cytokines mixture-based NK cell expansion}

RPMI medium is the most common medium to combine the NK cell expansion and CAR-NK generation. SCGM and NK-MACS medium are also widespread used in CARNK cell generation. However, all these media have different outcomes on final yield and possibly quality. When the highest cell numbers are required, it's wise to invest in comparison of different media and cytokine combinations. Cytokine-based NK cell expansion is in principle a feeder free expansion system, and is the most common used and the earliest method to expand NK cells. This method is based on adding soluble cytokines IL-2, IL-15 or IL-18 mixtures in the medium to propagate NK cell ex vivo. The cytokines eliminate the potential risk of contamination from feeder cells - derived from tumor cells -, making this approach clean and feasible. However, the time it takes to reach clinically relevant numbers may be very long as exemplified by Lapteva et al. who showed that their cytokine mixture needed expansion of NK cells during 4 to 10 weeks. Using irradiated (hence not proliferating) feeder cells it was shown that it took 10-14 days to achieve the required cell numbers needed for clinical therapy [161]. Which are in the order of $10^{8}$ cells / kg patient. Another disadvantage is that soluble cytokines are unstable and consumed, additional cytokines should be added multiple times, making manufacturing complicated and expensive. Cytokines can also be combined with chemical compounds. Zoledronate with IL-2 could induce a 1000-fold expansion of natural killer cells derived from umbilical cord blood [163].

\subsection{K562 feeder cells transfected with costimulatory molecules for NK cell expansion}

To facilitate a more rapid and stronger NK cell expansion, MHC low or nonexpressing cell lines are chosen as feeder cells after modification with one or more costimulatory molecules, like IL-15, IL-21, 4-1BBL, and OX-40, expressed on their surface. In general, MHC molecules will dampen the killing capacity of NK cells. Thus, the MHC class I deficient K562 cell line seems the ideal cell line to activate signal transduction in NK cells resulting in their expansion. Parental K562 cells have been shown to be unable to trigger NK cell expansion, however, gene modified K562 cells with membrane bound IL-15, or IL-21 in combination with 41BB-L as feeder cells have been used to expand NK cells and successfully applied in the clinical setting [162]. The $721.221 \mathrm{~B}$ cell line expressing IL-21 on the cell surface have been proven with superior ability than K562 mIL-21 to induce NK cell expansion [164]. PC3 (PSCA)-derived feeder cells expressing IL-2, 4-1BBL and membrane-bound IL-15-mutDAP12 (mIL-15d) fusion protein in combinations or alone were also demonstrated to possess the ability to induce NK cell expansion [165] but have not been directly compared to the gene modified K562 and 721.211 cell lines. Feeder cells may also provide additional, but yet unidentified, signals positively influencing proliferation.

The advancement of NK cell expansion technologies also facilitates CAR-NK cell generation. After 7 days expanded with K562-mlL-15/4-1BBL feeder cells, the transduction efficiency of freshly isolated NK cells can be above $80 \%$ compared to below $20 \%$ on freshly isolated NK cells [92]. In total, at this moment there are 13 studies published that used the K562 cells as feeder cells for CAR-NK cells to expand, including 10 studies using K562-mIL-15 feeder cells, 2 groups used K562-mIL-21 cells and 1 
publication used the parental K562 cell. NK cells expanded with mblL15 showed reduced telomere lengths, however, propagated with mbIL21 they had more elongated telomere lengths than freshly isolated peripheral blood NK cells [143] suggesting the mechanism by which these cells can be expanded over many generations to reach clinically relevant cell numbers.

\section{3 |lL-2, OKT3 plus autologous irradiated PBMC}

Irradiated autologous PBMC have been used to expand NK cells for long time $[166,167]$. Compared with freshly isolated NK cells, PBMC feeder cell based expanded NK cells manifested a robust CD107a activation accompanied by a high killing activity [168]. As the feeder cells are proliferating cells, they have to be irradiated to prevent further cell division (and reducing risk). This does not affect their supportive capacity as after irradiation these autologous cells are combined with IL-2 and the OKT3 antibody, and manifested strong NK cell expansion ability. This approach has been applied to generate NK cells for clinical trials [169]. After three weeks, the NK cell expansion could achieve $10^{4}$ times. This is biologically relevant but for large scale production the time it takes is too long and thus not commercially and practically interesting.

\subsection{L-15 agonist in vivo expansion}

In this era of continuous technology advancements, NK cell expansion has also been exposed to different new approaches. In contrast to expensive ex vivo generation and expansion of NK cells, in vivo NK cell expansion could be ideal from a manufacturing point of view. One example is the reagent IL-15 super agonist (ALT-803) that has been demonstrated to increasing the infiltration of NK cells and CD8+ T cells without increasing the number of Treg cells in a humanized mouse model $[170,171]$. In the Phase I clinical trial of ALT-803, head and neck, melanoma, lung advanced solid tumor patients were administrated through intravenous and subcutaneous [172]. NK cells population expanded after administrated with this agonist in all patients. These tumor patients showed some adverse effects including fatigue and nausea most commonly with intravenous administration, whereas painful injection site wheal was reported most commonly with subcutaneous injection ALT-803 [172]. In human, however, the disadvantage of this in vivo induced expansion may be a lack of activation as no mismatch is present. See for further discussion on in vivo targeting of NK cells paragraph 8.2.

\section{Prospective and outlook}

\subsection{Progression in CAR-NK clinical trials}

The number of clinical trials in the CAR-NK cell space is limited (Table 7). Although in recent years, the first 2 papers showing evidence of clinical successes have been published $[30,173]$, many of the investigations are still in early planning or recruiting into clinical trials. Most of these trials are company-driven, with the majority coming from China (Table 7). In these trials, various diseases are targeted, with an anti-CD19 CAR (B cell malignancies) on NK cells initially being compared the same CAR on T cells. Furthermore, different tumor antigens, like HER2, Mucin-1, EpCAM, or PMSA are targeted on a variety of solid and hematological tumors (Table 7). In the clinical studies currently conducted, CAR-NK cells are derived from PBMC, the NK-92 cell line or a 
newly generated uniform cell line NK101. In contrast, Rezvani and colleagues have used expanded umbilical cord NK cells, selected on a KIR. Mismatch between donor and recipient and promising results were reported [30].

The treatment was safe as no cytokine release syndrome, neurotoxicity, or GVHD occurred. From the 11 treated patients, 8 responded with 7 patients going into complete remission, although 3 patients had minimal residual disease at final assessment. CARNK cells were tracked in vivo and could be detected rather stable over time and for at least 12 months. It was suggested that in vivo persistence was driven by proliferation of the CAR-NK cells, which could be caused by the addition of IL-15 in the CAR construct (hence one could define this as a fourth generation CAR (see section 2.7 and Table 3 ). At least in mice it has been shown that IL-15 aids in the persistence of (dual-switch CD123 or BCMA) CAR-NK cells for minimal 40-50 days [69], when the experiments were stopped.

In addition, the first patient treated with iPSC-derived CAR-NK cells has recently been reported, also suggesting that CAR-NK cells can outperform CAR-T cells $[28,174]$. Still, the clinical trial is ongoing and the results have to be awaited. While many questions regarding CAR-NK cell therapy in humans remain, the large number of preclinical investigations imply that it is only a matter of time before more clinical trials are conducted.

\subsection{Technological advances in CAR-NK generation: biomaterials and in vivo transduction}

CAR-NK development was built on the knowledge gained from the CAR-T field. To further the development of CAR-NK for clinical use, technological advances will importantly speed up the process. Here, we consider two developments that will aid in the generation of sufficient CAR-NK cells for clinical use and for the in vivo generation of CAR-NK cells.

Biomaterials have shown promise as a concomitant agent together with effector cells for immunotherapy that can aid in mounting a stronger response, while simultaneously lowering the risk for side-effects [175]. In NK cells, biomaterial micelles are able to facilitate the formation of an immune synapse with tumor cells, aiding in the eradication of solid malignant lumps in vivo [176]. A three-dimensional engineered hyaluronic acidbased niche (3D-ENHANCE), instead of the classic two-dimensional culture methods, was shown to promote CAR-NK cell expansion and to enhance cytokine release, while maintaining killing capacity of EGFR-CAR-NK92 towards solid tumors in vivo [177]. In another study, biomaterial-modified Fc fragments on antibodies showed a strong potential to broaden NK cell recognition of heterogeneous antigens on solid tumors [178]. Moreover, Smit et al. reported that DNA-carrying nanoparticles can efficiently introduce leukemia-targeting CAR genes into T-cells [179], which was also reported for NK-92MI cells in vitro [180]. Thus, various promising inventions from the bio-engineering field are likely to enter the CAR field as important improvements in the design and engineering of cancer immunotherapy.

Various groups are trying to harness autologous lymphocytes, including NK cells for immunotherapy without the need for ex vivo modifications [181]. This presents various challenges, such as achieving proper activation of NK cells without haplotype-dependent, allogeneic activation. Furthermore, in vivo proliferation and maintenance is required to obtain sufficient cell numbers to mount a successful anti-tumor response. Recently, it has been shown that the engineering of the glycoprotein on the VSV-G virus with antiCD8 scFv makes it possible to specifically transduce CD8 T cells in vivo [181]. Using 
this concept, Pfeiffer et al. showed another approach of in vivo CAR-T generation by injecting lentivirus directly into laboratory mice. Indeed, in vivo injection of the CD8 specific-CD19 CAR-encoding lentiviruses resulted in transduction solely of the CD8+ T cells with a CD19-CAR [182]. However, this method also led to signs of a cytokine release syndrome, thus warranting further optimization before this in vivo CAR-T generation concept could be implemented in the clinic. These methods have not been tried on NK cells; we envisage that the concepts of these techniques can easily be applied to CAR-NK cell generation as well.

\subsection{Challenges}

Still many aspects on CAR constructs will have to be improved and many challenges lie ahead of the field. Besides the constructs, other aspects have to be taken into account. Here, we discuss three important challenges for the CAR-NK field that will need to be addressed in the near future regarding clinical safety, cryopreservation of CAR-NK cells and challenges posed by the tumor microenvironment.

The potential of CAR-based therapy has been clearly demonstrated, but it is not without risks. Indeed, patients treated with CAR-T cells have died because of cytokine release syndrome and off-target cytotoxic effects [1,183]. While improvements in CAR design have made CAR-based therapy safer by reducing these severe side-effects $[31,184]$, the incorporation of safety switches that quickly eliminate the CAR-NK infused cells, provides another level of safety $[69,185]$. CAR-NK cells are in general considered safer than CAR-T cells, although there is very limited clinical experience with CAR-NK therapy in patients, justifying additional safety measures, including the use of "suicide genes" [117].

Indeed, inducible caspase 9 (iCasp9) was included as a safety switch in the first reported clinical trial of CAR-NK [30]. While no severe side-effects warranting the need to use this safety switch were reported, it might be necessary to use it at a later time point, since CAR-NK cells were still detected after one year in vivo. The inducible safety switch caspase 9 (iCasp9) suicide gene is a modified caspase 9 gene fused to the human FK506 binding protein gene (FKBP). Addition of the inducer of dimerization (CID) (AP1903) leads to dimerization and recruits the downstream caspase molecules, thereby initiating apoptosis [186].

Recently, Ruella et al. demonstrated that anti-CAR19 idiotype chimeric antigen receptor (aCAR19) cells could eliminate CAR19+ T cells [187]. This CAR-T eliminating other CAR-T cells is not only useful for eliminating infused cells in the case of side-effects, but also at the same time it depletes all transduced cells (including leukemic blasts) present during CAR-T manufacturing, minimizing the potential clinical effect, but preventing side effects. Additionally, these cells can be used to deplete CAR-T cells to reduce long-term side effects, such as $B$ cell aplasia in the case of CD19-targeting CARs.

Truncated EGFR [188], CD19, CD20 or CD34 epitopes together with the CAR gene have been developed for CAR-T therapies [189]. Once the CAR-transduced cells cause undesirable effects, an additional administration of a specific antibody against these epitopes will induce ADCC to eliminate the CAR-NK cells in vivo $[29,69,190,191]$.

Not all issues related to the use of lentivirus for clinical use have already been resolved. For instance, it has been demonstrated that lentivirus-mediated p53 activation can lead to delayed cell proliferation, cell cycle arrest and slightly but significantly increased apoptosis during cell culture [192]. In a study using autologous CAR-T manufacturing, it was reported that the CAR gene was inadvertently transduced into a single leukemic B cell. The anti-CD19-scFv combined with the CD19 molecule on the 
surface of all leukemic B cells derived from this clone, camouflaging it from recognition by the CAR-T and becoming insensitive to the aimed effect [193]. Another case illustrated a patient suffering from chronic lymphocytic leukemia, in which after infusion with engineered autologous T cells, $94 \%$ of the CAR-T cells were found to originate from a single clone in which the lentiviral vector with the CAR transgene disrupted the methylcytosine dioxygenase TET2 gene [194]. Fortunately, the TET2 disruption by the lentivirus integration altered the epigenetic profile of the T cells that resulted in enhanced differentiation and expansion and exerted a central memory phenotype [194].

Retrovirus-mediated gene transduction has been reported to lead to random insertions creating the risk of leukemia [195,196]. However, with advances in the development of viral vectors and detection technology, June's group published 17 vector lots, 375 manufactured T cell products, and 308 treated patients with both oncologic and HIV-related indications for treatment (2001-2016), showing no evidence of generation of replication-competent retroviruses or lentiviruses [197].

Genome editing by CRISPR-Cas9 has been reported to induce p53-mediated DNA damage responses and cell cycle arrest in human cells [198]. Moreover, the potential of pre-existing humoral and cell-mediated adaptive immune responses to the Cas 9 system in humans should be taken seriously when this system is applied in vivo during clinical trials [199]. All these issues will have to be addressed to guarantee the safe use of CAR$\mathrm{T}$ and CAR-NK in patients.

An important technical issue is the cryopreservation of NK cell. For clinical application and infusion of cells into patients, large-scale expansion of CAR-NK cells under Good Manufacturing Practice (GMP) is required by authorities. It is hardly possible that a freshly expanded CAR-NK cell product can be administered into patients at a suitable time point taking into account the status of the cells and the logistics and timing to prepare the patient [162]. Cryopreservation of CAR-NK cells is a necessity, but also allows using NK cells as "off-the-shelf" products. This measure should guarantee the recipient always receives a similar quality of CAR-NK cells, thus disregarding the natural variety in NK cell donors [200 ]. Numerous groups are trying to optimize the ingredients of the freezing medium and procedures for expanded CAR-NK cells and have achieved encouraging outcomes $[162,169,200]$. A recent approach uses nanoparticle-mediated intracellular protection of NK cells which can avoid cryoinjury and maintain the killing potential of NK-92 cells, which could completely replace DMSO as a freezing protector [201]. This technique still has to be tried on and optimized for primary NK cells.

Even if the best CAR-NK cells can be made, they always will encounter the suppressive tumor microenvironment, possibly the biggest challenge of all to overcome [202]. So far, much effort is put in to designing CARs or combinations of treatments in overcoming the negative effects of the TME. CARs have been combined with checkpoint inhibitors like PD-1 [203,204] and were successful in mice [203], but less in human [204]. Depletion of soluble suppressive factors like TGF- $\beta$, IDO and IL-4 can be obtained by expressing of dominant negative mutants or gene editing of the respective genes $[205,206]$. Many solid tumors are characterized by hypoxic cores which may influence the function of NK cells, or on the other hand using and an HIF1a-driven CAR one could take advantage of the hypoxic environment [207]. A reduction of immune suppressor cells that are abundantly present in the TME (Treg [208], MDSC [209], TAM [210]) also is directed towards an improved clinical outcome and increased overall survival. The 
challenges posed by the tumor microenvironment and possible solutions using CARengineered cells has been covered extensively in various recent reviews [202,211].

\section{Conclusion}

In this review, we performed a comprehensive analysis of pre-clinical and clinical studies from the CAR-NK field with an emphasis on the design of the CAR and engineering of NK cells. Recommendations for usage of gene elements have been described, as well as the state-of-the-art, including important lessons from CAR-T development. This detailed information will pave the way to more robust CAR-NK cells studies with the aim of getting a stronger anti-tumor response with fewer side-effects compared to CAR-T cells. Overcoming the suppressive tumor microenvironment remains an important challenge on the design of CARs before CAR-NK cells, in combination with other therapies, will fulfill their promise.

\section{Abbreviations}

ADCC: Antibody-dependent cellular cytotoxicity; ALL: Acute lymphoblastic leukemia; AML: Acute myeloid leukemia; APC: Antigen presenting cell; BCMA: B-cell maturation antigen; CAR: Chimeric antigen receptor; Cas9: CRISPR-associated system 9; CCR: CC chemokine receptor; CD: cluster of differentiation; CLL: Chronic lymphocytic leukemia; CR: Complete remission; CRS: Cytokine release syndrome; CRISPR: clustered regularly interspaced short palindromic repeats; DLBCL: Diffuse large B-cell lymphoma; EGFR: epidermal growth factor receptor; FasL: Fas ligand; FDA: US Food and Drug Administration; GM-CSF: Granulocyte-macrophage colony stimulating factor; GVHD: Graft vs. host disease; Her2: human epidermal growth factor receptor 2; HLA: Human leukocyte antigen; HSPC: Hematopoietic stem/progenitor cell; IFN-Y: InterferonY; iPSC: Induced pluripotent stem cell; IS: Immunologic synapse; ITAM: Immunoreceptor tyrosine-based activation motif; KIR: Killer immunoglobulin receptor; MHC: major histocompatibility complex; MoAb: Monoclonal antibody; MM: Multiple myeloma; MOI: Multiplicity of infection; NCR: Natural cytotoxicity receptor; NK: Natural killer; NSG: NOD scid gamma; PB: Peripheral blood; PR: partial remission PSMA: Prostate-specific membrane antigen; PSCA: Prostate stem cell antigen; RT-PCR: Real-time PCR; scFv: Single-chain antibody variable fragment; TAA: Tumor-associated antigen; TCR: T cell receptor; TGF- $\beta$ : Transforming growth factor-beta; TME: Tumor microenvironment; TRAIL: TNF-related apoptosis-inducing ligand; UCB: Umbilical cord blood. 


\section{Acknowledgements and Funding}

We thank Prof. Dr. Reno Debets for providing CAIX-CAR virus supernatant. We thank Prof. Dr. Winfried Wels for sending anti-CD19-CAR-cMyc lentivirial vector. We appreciate Prof. Dr. Els Verhoeyen sharing the BaEV lentivirus vector. We thank Prof. Dr. Dean Lee for giving the K-562-mIL-21-4-1BBL cell line.

Y.G. thanks the China Scholarship Council (CSC) (No. 201707720056) for fellowship support. R.G.J.K.W. is supported by a Kootstra Talent Fellowship (Maastricht University), a Marie Skłodowska-Curie Individual fellowship (799810-TOPNIN; European Union), a Cancer Research Institute/Irvington Postdoctoral Fellowship and a fellowship from the "la Caixa" Foundation (ID 100010434; LCF/BQ/PR20/11770004). This research study was supported by Cancer Research Foundation Limburg (2012-03 KOFL) and GROW School of Maastricht University.

We thank the Maastricht University CRISP Virus production core facility. 


\section{References}

1. June $\mathrm{CH}$, Sadelain M. Chimeric antigen receptor therapy. N Engl J Med. 2018; 379:64-73. doi:10.1056/NEJMra1706169.

2. Dunbar CE, High KA, Joung JK, Kohn DB, Ozawa K, Sadelain M. Gene therapy comes of age. Science. 2018; 359:eaan4672. doi:10.1126/science.aan4672.

3. Sordo-Bahamonde C, Vitale M. Mechanisms of resistance to nk cell immunotherapy. Cancers (Basel). 2020; 12:893. doi:10.3390/cancers12040893.

4. Weber EW, Maus MV, Mackall CL. The emerging landscape of immune cell therapies. Cell. 2020; 181:46-62. doi:https://doi.org/10.1016/j.cell.2020.03.001.

5. Melaiu O, Lucarini V, Cifaldi L, Fruci D. Influence of the tumor microenvironment on nk cell function in solid tumors. Front Immunol. 2020; 10:3038. doi:10.3389/fimmu.2019.03038.

6. Stoiber S, Cadilha BL, Benmebarek M-R, Lesch S, Endres S, Kobold S. Limitations in the design of chimeric antigen receptors for cancer therapy. Cells. 2019; 8:472. doi:10.3390/cells8050472.

7. Dotti G, Gottschalk S, Savoldo B, Brenner MK. Design and development of therapies using chimeric antigen receptor-expressing t cells. Immunol Rev. 2014; 257:107-126. doi:10.1111/imr.12131.

8. Wei J, Han X, Bo J, Han W. Target selection for car-t therapy. J Hematol Oncol. 2019; $12: 62$. doi:10.1186/s13045-019-0758-x.

9. Fleischer LC, Spencer HT, Raikar SS. Targeting $\mathrm{t}$ cell malignancies using car-based immunotherapy: Challenges and potential solutions. J Hematol Oncol. 2019; 12:141. doi:10.1186/s13045-019-0801-y.

10. Walsh Z, Yang Y, Kohler ME. Immunobiology of chimeric antigen receptor t cells and novel designs. Immunol Rev. 2019; 290:100-113. doi:10.1111/imr.12794.

11. Wang W, Jiang J, Wu C. Car-nk for tumor immunotherapy: Clinical transformation and future prospects. Cancer Lett. 2020; 472:175-180. doi:https://doi.org/10.1016/j.canlet.2019.11.033.

12. Pfefferle A, Huntington ND. You have got a fast car: Chimeric antigen receptor nk cells in cancer therapy. Cancers (Basel). 2020; 12:706

13. Burger MC, Zhang C, Harter PN, Romanski A, Strassheimer F, Senft C, et al. Car-engineered nk cells for the treatment of glioblastoma: Turning innate effectors into precision tools for cancer immunotherapy. Front Immunol. 2019; 10:2683. doi:10.3389/fimmu.2019.02683.

14. Hu Y, Tian Z, Zhang C. Chimeric antigen receptor (car)-transduced natural killer cells in tumor immunotherapy. Acta Pharmacol Sin. 2018; 39:167-176. doi:10.1038/aps.2017.125.

15. Shimasaki N, Jain A, Campana D. Nk cells for cancer immunotherapy. Nat Rev Drug Discov. 2020; 19:200-218. doi:10.1038/s41573-019-0052-1.

16. Klichinsky M, Ruella M, Shestova O, Lu XM, Best A, Zeeman M, et al. Human chimeric antigen receptor macrophages for cancer immunotherapy. Nat Biotechnol. 2020; 38:947-953. doi:10.1038/s41587-020-0462-y.

17. June $\mathrm{CH}$, O'Connor RS, Kawalekar OU, Ghassemi S, Milone MC. Car t cell immunotherapy for human cancer. Science. 2018; 359:1361-1365. doi:10.1126/science.aar6711.

18. Fang F, Xiao W, Tian Z. Challenges of nk cell-based immunotherapy in the new era. J Frontiers of Medicine. 2018; 12:440-450. doi:10.1007/s11684-018-0653-9.

19. Hu Y, Tian Z, Zhang C. Natural killer cell-based immunotherapy for cancer: Advances and prospects. Engineering. 2019; 5:106-114. doi:https://doi.org/10.1016/j.eng.2018.11.015.

20. Carotta S. Targeting nk cells for anticancer immunotherapy: Clinical and preclinical approaches. Front Immunol. 2016; 7:152. doi:10.3389/fimmu.2016.00152.

21. He Y, Tian Z. Nk cell education via nonclassical mhc and non-mhc ligands. Cell Mol Immunol. 2017; 14:321-330. doi:10.1038/cmi.2016.26.

22. Orr MT, Lanier LL. Natural killer cell education and tolerance. Cell. 2010; 142:847-856. doi:https://doi.org/10.1016/j.cell.2010.08.031.

23. Miller JS, Soignier Y, Panoskaltsis-Mortari A, McNearney SA, Yun GH, Fautsch SK, et al. Successful adoptive transfer and in vivo expansion of human haploidentical nk cells in patients with cancer. Blood. 2005; 105:3051-3057. doi:10.1182/blood-2004-07-2974.

24. Ruggeri L, Capanni M, Urbani E, Perruccio K, Shlomchik WD, Tosti A, et al. Effectiveness of donor natural killer cell alloreactivity in mismatched hematopoietic transplants. Science. 2002; 295:20972100. doi:10.1126/science.1068440.

25. Simonetta F, Alvarez M, Negrin RS. Natural killer cells in graft-versus-host-disease after allogeneic hematopoietic cell transplantation. Front Immunol. 2017; 8:465. doi:10.3389/fimmu.2017.00465.

26. Huang R-S, Lai M-C, Shih H-A, Lin S. A robust platform for expansion and genome editing of primary human natural killer cells. J Exp Med. 2021; 218:e20201529. doi:10.1084/jem.20201529. 
27. Suen WC-W, Lee WY-W, Leung K-T, Pan X-H, Li G. Natural killer cell-based cancer immunotherapy: A review on 10 years completed clinical trials. Cancer Investigation. 2018; 36:431-457. doi:10.1080/07357907.2018.1515315.

28. Li Y, Hermanson DL, Moriarity BS, Kaufman DS. Human ipsc-derived natural killer cells engineered with chimeric antigen receptors enhance anti-tumor activity. Cell Stem Cell. 2018; 23:181-192. doi:10.1016/j.stem.2018.06.002.

29. Quintarelli C, Sivori S, Caruso S, Carlomagno S, Falco M, Boffa I, et al. Efficacy of third-party chimeric antigen receptor modified peripheral blood natural killer cells for adoptive cell therapy of bcell precursor acute lymphoblastic leukemia. Leukemia. 2020; 34:1102-1115. doi:10.1038/s41375019-0613-7.

30. Liu E, Marin D, Banerjee P, Macapinlac HA, Thompson P, Basar R, et al. Use of car-transduced natural killer cells in cd19-positive lymphoid tumors. N Engl J Med. 2020; 382:545-553. doi:10.1056/NEJMoa1910607.

31. Ying Z, Huang XF, Xiang X, Liu Y, Kang X, Song $Y$, et al. A safe and potent anti-cd19 car $t$ cell therapy. Nat Med. 2019; 25:947-953. doi:10.1038/s41591-019-0421-7.

32. Allan DSJ, Chakraborty M, Waller GC, Hochman MJ, Poolcharoen A, Reger RN, et al. Systematic improvements in lentiviral transduction of primary human natural killer cells undergoing ex vivo expansion. Mol Ther Methods Clin Dev. 2021; 20:559-571. doi:10.1016/j.omtm.2021.01.008.

33. Kulemzin SV, Matvienko DA, Sabirov AH, Sokratyan AM, Chernikova DS, Belovezhets TN, et al. Design and analysis of stably integrated reporters for inducible transgene expression in human $t$ cells and car nk-cell lines. BMC Medical Genomics. 2019; 12:44. doi:10.1186/s12920-019-0489-4.

34. Milone MC, Fish JD, Carpenito C, Carroll RG, Binder GK, Teachey D, et al. Chimeric receptors containing cd137 signal transduction domains mediate enhanced survival of $t$ cells and increased antileukemic efficacy in vivo. Mol Ther. 2009; 17:1453-1464. doi:10.1038/mt.2009.83.

35. Jones S, Peng PD, Yang S, Hsu C, Cohen CJ, Zhao Y, et al. Lentiviral vector design for optimal t cell receptor gene expression in the transduction of peripheral blood lymphocytes and tumorinfiltrating lymphocytes. Hum Gene Ther. 2009; 20:630-640. doi:10.1089/hum.2008.048.

36. Fujiwara K, Masutani M, Tachibana M, Okada N. Impact of scfv structure in chimeric antigen receptor on receptor expression efficiency and antigen recognition properties. Biochem Biophys Res Commun. 2020; 527:350-357. doi:https://doi.org/10.1016/j.bbrc.2020.03.071.

37. Rad S M AH, Poudel A, Tan GMY, McLellan AD. Promoter choice: Who should drive the car in $\mathrm{t}$ cells? PLoS One. 2020; 15:e0232915-e0232915. doi:10.1371/journal.pone.0232915.

38. Zimmermann K, Kuehle J, Dragon AC, Galla M, Kloth C, Rudek LS, et al. Design and characterization of an "all-in-one" lentiviral vector system combining constitutive anti-gd2 car expression and inducible cytokines. Cancers (Basel). 2020; 12:375

39. Li W, Qiu S, Chen J, Jiang S, Chen W, Jiang J, et al. Chimeric antigen receptor designed to prevent ubiquitination and downregulation showed durable antitumor efficacy. Immunity. 2020; 53:456-470. doi:10.1016/j.immuni.2020.07.011.

40. Eyquem J, Mansilla-Soto J, Giavridis T, van der Stegen SJ, Hamieh M, Cunanan KM, et al. Targeting a car to the trac locus with crispr/cas9 enhances tumour rejection. Nature. 2017; 543:113-117. doi:10.1038/nature21405.

41. Torikai H, Reik A, Liu PQ, Zhou Y, Zhang L, Maiti S, et al. A foundation for universal t-cell based immunotherapy: $T$ cells engineered to express a cd19-specific chimeric-antigen-receptor and eliminate expression of endogenous tcr. Blood. 2012; 119:5697-5705. doi:10.1182/blood-2012-01405365.

42. Nguyen DN, Roth TL, Li PJ, Chen PA, Apathy R, Mamedov MR, et al. Polymer-stabilized cas9 nanoparticles and modified repair templates increase genome editing efficiency. Nat Biotechnol. 2020; 38:44-49. doi:10.1038/s41587-019-0325-6.

43. Pomeroy EJ, Hunzeker JT, Kluesner MG, Lahr WS, Smeester BA, Crosby MR, et al. A genetically engineered primary human natural killer cell platform for cancer immunotherapy. Mol Ther. 2020; 28:52-63. doi:https://doi.org/10.1016/j.ymthe.2019.10.009.

44. Owji H, Nezafat N, Negahdaripour M, Hajiebrahimi A, Ghasemi Y. A comprehensive review of signal peptides: Structure, roles, and applications. Eur J Cell Biol. 2018; 97:422-441. doi:https://doi.org/10.1016/j.ejcb.2018.06.003.

45. Nyathi Y, Wilkinson BM, Pool MR. Co-translational targeting and translocation of proteins to the endoplasmic reticulum. Biochim Biophys Acta. 2013; 1833:2392-2402. doi:10.1016/j.bbamcr.2013.02.021.

46. Voss M, Schröder B, Fluhrer R. Mechanism, specificity, and physiology of signal peptide peptidase (spp) and spp-like proteases. Biochimica et Biophysica Acta (BBA) - Biomembranes. 2013; 1828:2828-2839. doi:https://doi.org/10.1016/j.bbamem.2013.03.033. 
47. Haso W, Lee DW, Shah NN, Stetler-Stevenson M, Yuan CM, Pastan IH, et al. Anti-cd22-chimeric antigen receptors targeting b-cell precursor acute lymphoblastic leukemia. Blood. 2013; 121:11651174. doi:10.1182/blood-2012-06-438002.

48. Wilkie S, Picco G, Foster J, Davies DM, Julien S, Cooper L, et al. Retargeting of human t cells to tumor-associated muc1: The evolution of a chimeric antigen receptor. J Immunol. 2008; 180:49014909. doi:10.4049/jimmunol.180.7.4901.

49. Posey AD, Schwab RD, Boesteanu AC, Steentoft C, Mandel U, Engels B, et al. Engineered car $t$ cells targeting the cancer-associated tn-glycoform of the membrane mucin muc1 control adenocarcinoma. Immunity. 2016; 44:1444-1454. doi:10.1016/j.immuni.2016.05.014.

50. Morgan RA, Yang JC, Kitano M, Dudley ME, Laurencot CM, Rosenberg SA. Case report of a serious adverse event following the administration of $t$ cells transduced with a chimeric antigen receptor recognizing erbb2. Mol Ther. 2010; 18:843-851. doi:10.1038/mt.2010.24.

51. Lamers $\mathrm{CH}$, Sleijfer S, Vulto AG, Kruit WH, Kliffen M, Debets R, et al. Treatment of metastatic renal cell carcinoma with autologous t-lymphocytes genetically retargeted against carbonic anhydrase ix: First clinical experience. J Clin Oncol. 2006; 24:e20-22. doi:10.1200/jco.2006.05.9964.

52. Krokhotin A, Du H, Hirabayashi K, Popov K, Kurokawa T, Wan X, et al. Computationally guided design of single-chain variable fragment improves specificity of chimeric antigen receptors. Mol Ther Oncolytics. 2019; 15:30-37. doi:https://doi.org/10.1016/j.omto.2019.08.008.

53. Thokala R, Olivares S, Mi T, Maiti S, Deniger D, Huls H, et al. Redirecting specificity of t cells using the sleeping beauty system to express chimeric antigen receptors by mix-and-matching of $\mathrm{vl}$ and $\mathrm{vh}$ domains targeting cd123+ tumors. PLoS One. 2016; 11:e0159477. doi:10.1371/journal.pone.0159477.

54. Kang CH, Kim Y, Lee HK, Lee SM, Jeong HG, Choi SU, et al. Identification of potent cd19 scfv for car $\mathrm{t}$ cells through scfv screening with nk/t-cell line. Int $\mathrm{J}$ Mol Sci. 2020; 21. doi:10.3390/ijms21239163.

55. Fernández de Larrea C, Staehr M, Lopez AV, Ng KY, Chen Y, Godfrey WD, et al. Defining an optimal dual-targeted car t-cell therapy approach simultaneously targeting bcma and gprc5d to prevent bcma escape-driven relapse in multiple myeloma. Blood Cancer Discovery. 2020; 1:146-154. doi:10.1158/2643-3230.bcd-20-0020.

56. Liu Q, Xu Y, Mou J, Tang K, Fu X, Li Y, et al. Irradiated chimeric antigen receptor engineered nk$92 \mathrm{mi}$ cells show effective cytotoxicity against cd19(+) malignancy in a mouse model. Cytotherapy. 2020; 22:552-562. doi:10.1016/j.jcyt.2020.06.003.

57. Xu Y, Liu Q, Zhong M, Wang Z, Chen Z, Zhang Y, et al. 2b4 costimulatory domain enhancing cytotoxic ability of anti-cd5 chimeric antigen receptor engineered natural killer cells against $t$ cell malignancies. J Hematol Oncol. 2019; 12:49. doi:10.1186/s13045-019-0732-7.

58. Thomas S, Straathof K, Himoudi N, Anderson J, Pule M. An optimized gd2-targeting retroviral cassette for more potent and safer cellular therapy of neuroblastoma and other cancers. PLoS One. 2016; 11:e0152196. doi:10.1371/journal.pone.0152196.

59. Hege KM, Bergsland EK, Fisher GA, Nemunaitis JJ, Warren RS, McArthur JG, et al. Safety, tumor trafficking and immunogenicity of chimeric antigen receptor (car)-t cells specific for tag-72 in colorectal cancer. J Immunother Cancer. 2017; 5:22. doi:10.1186/s40425-017-0222-9.

60. Whitlow M, Bell BA, Feng SL, Filpula D, Hardman KD, Hubert SL, et al. An improved linker for singlechain $\mathrm{fv}$ with reduced aggregation and enhanced proteolytic stability. Protein Eng. 1993; 6:989-995. doi:10.1093/protein/6.8.989.

61. Alabanza L, Pegues M, Geldres C, Shi V, Wiltzius JJW, Sievers SA, et al. Function of novel anticd19 chimeric antigen receptors with human variable regions is affected by hinge and $\begin{array}{llll}\text { transmembrane } & \text { domains. } & \text { Mol 2017; } & \text { Ther. }\end{array}$ doi:https://doi.org/10.1016/j.ymthe.2017.07.013.

62. Hudecek M, Sommermeyer D, Kosasih PL, Silva-Benedict A, Liu L, Rader C, et al. The nonsignaling extracellular spacer domain of chimeric antigen receptors is decisive for in vivo antitumor activity. Cancer Immunol Res. 2015; 3:125-135. doi:10.1158/2326-6066.cir-14-0127.

63. Guo C, Wang X, Zhang H, Zhi L, Lv T, Li M, et al. Structure-based rational design of a novel chimeric pd1-nkg2d receptor for natural killer cells. Mol Immunol. 2019; 114:108-113. doi:https://doi.org/10.1016/j.molimm.2019.07.009.

64. Gotthardt D, Trifinopoulos J, SexI V, Putz EM. Jak/stat cytokine signaling at the crossroad of nk cell development and maturation. Front Immunol. 2019; 10:2590. doi:10.3389/fimmu.2019.02590.

65. Lanier LL. Up on the tightrope: Natural killer cell activation and inhibition. Nat Immunol. 2008; 9:495502. doi:10.1038/ni1581.

66. Lindner SE, Johnson SM, Brown CE, Wang LD. Chimeric antigen receptor signaling: Functional consequences and design implications. Sci Adv. 2020; 6:eaaz3223. doi:10.1126/sciadv.aaz3223. 
67. MacKay M, Afshinnekoo E, Rub J, Hassan C, Khunte M, Baskaran N, et al. The therapeutic landscape for cells engineered with chimeric antigen receptors. Nat Biotechnol. 2020; 38:233-244. doi:10.1038/s41587-019-0329-2.

68. Nowakowska P, Romanski A, Miller N, Odendahl M, Bonig H, Zhang C, et al. Clinical grade manufacturing of genetically modified, car-expressing nk-92 cells for the treatment of erbb2-positive malignancies. Cancer Immunol Immunother. 2018; 67:25-38. doi:10.1007/s00262-017-2055-2.

69. Wang X, Jasinski DL, Medina JL, Spencer DM, Foster AE, Bayle JH. Inducible myd88/cd40 synergizes with il-15 to enhance antitumor efficacy of car-nk cells. Blood Adv. 2020; 4:1950-1964. doi:10.1182/bloodadvances.2020001510.

70. Daher M, Basar R, Gokdemir E, Baran N, Uprety N, Nunez Cortes AK, et al. Targeting a cytokine checkpoint enhances the fitness of armored cord blood car-nk cells. Blood. 2021; 137:624-636. doi:10.1182/blood.2020007748.

71. Hammer Q, Rückert T, Borst EM, Dunst J, Haubner A, Durek P, et al. Peptide-specific recognition of human cytomegalovirus strains controls adaptive natural killer cells. Nat Immunol. 2018; 19:453463. doi:10.1038/s41590-018-0082-6.

72. Romee R, Schneider SE, Leong JW, Chase JM, Keppel CR, Sullivan RP, et al. Cytokine activation induces human memory-like nk cells. Blood. 2012; 120:4751-4760. doi:10.1182/blood-2012-04419283.

73. Romee R, Rosario M, Berrien-Elliott MM, Wagner JA, Jewell BA, Schappe T, et al. Cytokine-induced memory-like natural killer cells exhibit enhanced responses against myeloid leukemia. Sci Transl Med. 2016; 8:357ra123. doi:10.1126/scitranslmed.aaf2341.

74. Salter AI, Ivey RG, Kennedy JJ, Voillet V, Rajan A, Alderman EJ, et al. Phosphoproteomic analysis of chimeric antigen receptor signaling reveals kinetic and quantitative differences that affect cell function. Sci Signal. 2018; 11:eaat6753. doi:10.1126/scisignal.aat6753.

75. Zhao Y, Wang QJ, Yang S, Kochenderfer JN, Zheng Z, Zhong X, et al. A herceptin-based chimeric antigen receptor with modified signaling domains leads to enhanced survival of transduced $t$ lymphocytes and antitumor activity. J Immunol. 2009; 183:5563. doi:10.4049/jimmunol.0900447.

76. Feucht J, Sun J, Eyquem J, Ho Y-J, Zhao Z, Leibold J, et al. Calibration of car activation potential directs alternative $t$ cell fates and therapeutic potency. Nat Med. 2019; 25:82-88. doi:10.1038/s41591-018-0290-5.

77. Long AH, Haso WM, Shern JF, Wanhainen KM, Murgai M, Ingaramo M, et al. 4-1bb costimulation ameliorates $t$ cell exhaustion induced by tonic signaling of chimeric antigen receptors. Nat Med. 2015; 21:581-590. doi:10.1038/nm.3838.

78. Ajina A, Maher J. Strategies to address chimeric antigen receptor tonic signaling. Molecular Cancer Therapeutics. 2018; 17:1795-1815. doi:10.1158/1535-7163.Mct-17-1097.

79. Kawalekar OU, O'Connor RS, Fraietta JA, Guo L, McGettigan SE, Posey AD, Jr., et al. Distinct signaling of coreceptors regulates specific metabolism pathways and impacts memory development in car t cells. Immunity. 2016; 44:380-390. doi:10.1016/j.immuni.2016.01.021.

80. Gang M, Marin ND, Wong P, Neal CC, Marsala L, Foster M, et al. Car-modified memory-like nk cells exhibit potent responses to nk-resistant lymphomas. Blood. 2020; 136:2308-2318. doi:10.1182/blood.2020006619.

81. Gudipati V, Rydzek J, Doel-Perez I, Gonçalves VDR, Scharf L, Königsberger S, et al. Inefficient carproximal signaling blunts antigen sensitivity. Nat Immunol. 2020; 21:848-856. doi:10.1038/s41590020-0719-0.

82. Oelsner S, Friede ME, Zhang C, Wagner J, Badura S, Bader P, et al. Continuously expanding car nk-92 cells display selective cytotoxicity against b-cell leukemia and lymphoma. Cytotherapy. 2017; 19:235-249. doi:10.1016/j.jcyt.2016.10.009.

83. Chu J, Deng Y, Benson DM, He S, Hughes T, Zhang J, et al. Cs1-specific chimeric antigen receptor (car)-engineered natural killer cells enhance in vitro and in vivo antitumor activity against human multiple myeloma. Leukemia. 2014; 28:917-927. doi:10.1038/leu.2013.279.

84. Chen F, Wu P, Deng S, Zhang H, Hou Y, Hu Z, et al. Dissimilation of synonymous codon usage bias in virus-host coevolution due to translational selection. Nat Ecol Evol. 2020; 4:589-600. doi:10.1038/s41559-020-1124-7.

85. Schutsky K, Song D-G, Lynn R, Smith JB, Poussin M, Figini M, et al. Rigorous optimization and validation of potent rna car $t$ cell therapy for the treatment of common epithelial cancers expressing folate receptor. Oncotarget. 2015; 6:

86. Qian L, Li D, Ma L, He T, Qi F, Shen J, et al. The novel anti-cd19 chimeric antigen receptors with humanized scfv (single-chain variable fragment) trigger leukemia cell killing. Cell Immunol. 2016; 304-305:49-54. doi:https://doi.org/10.1016/j.cellimm.2016.03.003. 
87. Schuster SJ, Bishop MR, Tam CS, Waller EK, Borchmann P, McGuirk JP, et al. Tisagenlecleucel in adult relapsed or refractory diffuse large b-cell lymphoma. N Engl J Med. 2018; 380:45-56. doi:10.1056/NEJMoa1804980.

88. June $\mathrm{CH}$, Blazar BR, Riley JL. Engineering lymphocyte subsets: Tools, trials and tribulations. Nat Rev Immunol. 2009; 9:704-716. doi:10.1038/nri2635.

89. Milone MC, O'Doherty U. Clinical use of lentiviral vectors. Leukemia. 2018; 32:1529-1541. doi:10.1038/s41375-018-0106-0.

90. Gándara C, Affleck V, Stoll EA. Manufacture of third-generation lentivirus for preclinical use, with process development considerations for translation to good manufacturing practice. Hum Gene Ther Methods. 2018; 29:1-15. doi:10.1089/hgtb.2017.098.

91. Müller S, Bexte T, Gebel V, Kalensee F, Stolzenberg E, Hartmann J, et al. High cytotoxic efficiency of lentivirally and alpharetrovirally engineered cd19-specific chimeric antigen receptor natural killer cells against acute lymphoblastic leukemia. Front Immunol. 2020; 10:3123. doi:10.3389/fimmu.2019.03123.

92. Colamartino ABL, Lemieux W, Bifsha P, Nicoletti S, Chakravarti N, Sanz J, et al. Efficient and robust nk-cell transduction with baboon envelope pseudotyped lentivector. Front Immunol. 2019; 10:2873. doi:10.3389/fimmu.2019.02873.

93. Gong Y, Klein Wolterink RGJ, Janssen I, Groot AJ, Bos GMJ, Germeraad WTV. Rosuvastatin enhances vsv-g lentiviral transduction of $\mathrm{nk}$ cells via upregulation of the low-density lipoprotein receptor. Mol Ther Methods Clin Dev. 2020; 17:634-646. doi:10.1016/j.omtm.2020.03.017.

94. Bari R, Granzin M, Tsang KS, Roy A, Krueger W, Orentas R, et al. A distinct subset of highly proliferative and lentiviral vector (Iv)-transducible nk cells define a readily engineered subset for adoptive cellular therapy. Front Immunol. 2019; 10:2001. doi:10.3389/fimmu.2019.02001.

95. Suerth JD, Labenski V, Schambach A. Alpharetroviral vectors: From a cancer-causing agent to a useful tool for human gene therapy. Viruses. 2014; 6:4811-4838. doi:10.3390/v6124811.

96. Poletti V, Mavilio F. Interactions between retroviruses and the host cell genome. Mol Ther Methods Clin Dev. 2018; 8:31-41. doi:10.1016/j.omtm.2017.10.001.

97. Suerth JD, Morgan MA, Kloess S, Heckl D, Neudorfl C, Falk CS, et al. Efficient generation of genemodified human natural killer cells via alpharetroviral vectors. J Mol Med (Berl). 2016; 94:83-93. doi:10.1007/s00109-015-1327-6.

98. Carlsten M, Childs RW. Genetic manipulation of nk cells for cancer immunotherapy: Techniques and clinical implications. Front Immunol. 2015; 6:266. doi:10.3389/fimmu.2015.00266.

99. Chu Y, Yahr A, Huang B, Ayello J, Barth M, S. Cairo M. Romidepsin alone or in combination with anti-cd20 chimeric antigen receptor expanded natural killer cells targeting burkitt lymphoma in vitro and in immunodeficient mice. Oncolmmunology. 2017; 6:e1341031. doi:10.1080/2162402X.2017.1341031.

100. Shimasaki N, Fujisaki H, Cho D, Masselli M, Lockey T, Eldridge P, et al. A clinically adaptable method to enhance the cytotoxicity of natural killer cells against b-cell malignancies. Cytotherapy. 2012; 14:830-840. doi:https://doi.org/10.3109/14653249.2012.671519.

101. Batchu RB, Gruzdyn OV, Tavva PS, Kolli BK, Dachepalli R, Weaver DW, et al. Engraftment of mesothelin chimeric antigen receptor using a hybrid sleeping beauty/minicircle vector into nk-92mi cells for treatment of pancreatic cancer. Surgery. 2019; 166:503-508. doi:https://doi.org/10.1016/j.surg.2019.05.047.

102. Wang J, Lupo KB, Chambers AM, Matosevic SJJfloC. Purinergic targeting enhances immunotherapy of cd73+ solid tumors with piggybac-engineered chimeric antigen receptor natural killer cells. J Immunother Cancer. 2018; 6:136. doi:10.1186/s40425-018-0441-8.

103. Hudecek M, Izsvák Z, Johnen S, Renner M, Thumann G, Ivics Z. Going non-viral: The sleeping beauty transposon system breaks on through to the clinical side. Critical Reviews in Biochemistry and Molecular Biology. 2017; 52:355-380. doi:10.1080/10409238.2017.1304354.

104. Gurney M, Stikvoort A, Nolan E, Kirkham-McCarthy L, Khoruzhenko S, Shivakumar R, et al. Cd38 knockout natural killer cells expressing an affinity optimized cd38 chimeric antigen receptor successfully target acute myeloid leukemia with reduced effector cell fratricide. Haematologica. 2020. doi:10.3324/haematol.2020.271908.

105. Afolabi LO, Adeshakin AO, Sani MM, Bi J, Wan X. Genetic reprogramming for nk cell cancer immunotherapy with crispr/cas9. Immunology. 2019; 158:63-69. doi:10.1111/imm.13094.

106. Bailey SR, Maus MV. Gene editing for immune cell therapies. Nat Biotechnol. 2019; 37:1425-1434. doi:10.1038/s41587-019-0137-8.

107. Stenger D, Stief TA, Käuferle T, Willier S, Rataj F, Schober K, et al. Endogenous tcr promotes in vivo persistence of cd19-car-t cells compared to a crispr/cas9-mediated tcr knockout car. Blood. 2020; 136:1407-1418. doi:10.1182/blood.2020005185. 
108. De Oliveira SN, Wang J, Ryan C, Morrison SL, Kohn DB, Hollis RP. A cd19/fc fusion protein for detection of anti-cd19 chimeric antigen receptors. J Transl Med. 2013; 11:23. doi:10.1186/14795876-11-23.

109. Nilson BH, Solomon A, Björck L, Akerström B. Protein I from peptostreptococcus magnus binds to the kappa light chain variable domain. J Biol Chem. 1992; 267:2234-2239

110. Zheng Z, Chinnasamy N, Morgan RA. Protein I: A novel reagent for the detection of chimeric antigen receptor (car) expression by flow cytometry. J Transl Med. 2012; 10:29. doi:10.1186/1479-5876-1029.

111. Zhang C, Oberoi P, Oelsner S, Waldmann A, Lindner A, Tonn T, et al. Chimeric antigen receptorengineered nk-92 cells: An off-the-shelf cellular therapeutic for targeted elimination of cancer cells and induction of protective antitumor immunity. Front Immunol. 2017; 8:533. doi:10.3389/fimmu.2017.00533.

112. Chiarella P, Edelmann B, Fazio VM, Sawyer AM, de Marco A. Antigenic features of protein carriers commonly used in immunisation trials. Biotechnol Lett. 2010; 32:1215-1221. doi:10.1007/s10529010-0283-z.

113. Casucci M, Falcone L, Camisa B, Norelli M, Porcellini S, Stornaiuolo A, et al. Extracellular ngfr spacers allow efficient tracking and enrichment of fully functional car-t cells co-expressing a suicide gene. Front Immunol. 2018; 9:507. doi:10.3389/fimmu.2018.00507.

114. Härmälä SK, Butcher R, Roberts $\mathrm{CH}$. Copy number variation analysis by droplet digital pcr. Methods Mol Biol. 2017; 1654:135-149. doi:10.1007/978-1-4939-7231-9 9.

115. Pinheiro LB, Coleman VA, Hindson CM, Herrmann J, Hindson BJ, Bhat S, et al. Evaluation of a droplet digital polymerase chain reaction format for DNA copy number quantification. Anal Chem. 2012; 84:1003-1011. doi:10.1021/ac202578x.

116. Chang ZL, Lorenzini MH, Chen X, Tran U, Bangayan NJ, Chen YY. Rewiring t-cell responses to soluble factors with chimeric antigen receptors. Nat Chem Biol. 2018; 14:317-324. doi:10.1038/nchembio.2565.

117. Liu E, Tong Y, Dotti G, Shaim H, Savoldo B, Mukherjee M, et al. Cord blood nk cells engineered to express il-15 and a cd19-targeted car show long-term persistence and potent antitumor activity. Leukemia. 2018; 32:520-531. doi:10.1038/leu.2017.226.

118. Gangadaran P, Ahn B-C. Molecular imaging: A useful tool for the development of natural killer cellbased immunotherapies. Front Immunol. 2017; 8:1090. doi:10.3389/fimmu.2017.01090.

119. Gandara C, Affleck V, Stoll EA. Manufacture of third-generation lentivirus for preclinical use, with process development considerations for translation to good manufacturing practice. Hum Gene Ther Methods. 2018; 29:1-15

120. Joglekar AV, Sandoval. S. Pseudotyped lentiviral vectors: One vector, many guises. Hum Gene Ther Methods. 2017; 28:291-301. doi:10.1089/hgtb.2017.084.

121. Verhoeyen E, Cosset F-L. Surface-engineering of lentiviral vectors. J Gene Med. 2004; 6:S83-S94. doi:10.1002/jgm.494.

122. Finkelshtein D, Werman A, Novick D, Barak S, Rubinstein M. Ldl receptor and its family members serve as the cellular receptors for vesicular stomatitis virus. Proc Natl Acad Sci U S A. 2013; 110:7306-7311

123. Amirache F, Lévy C, Costa C, Mangeot P-E, Torbett BE, Wang CX, et al. Mystery solved: Vsv-g-Ivs do not allow efficient gene transfer into unstimulated $t$ cells, b cells, and hscs because they lack the Idl receptor. Blood. 2014; 123:1422-1424. doi:10.1182/blood-2013-11-540641.

124. Davis HE, Rosinski M, Morgan JR, Yarmush ML. Charged polymers modulate retrovirus transduction via membrane charge neutralization and virus aggregation. Biophysical Journal. 2004; 86:1234-1242. doi:https://doi.org/10.1016/S0006-3495(04)74197-1.

125. Davis HE, Morgan JR, Yarmush ML. Polybrene increases retrovirus gene transfer efficiency by enhancing receptor-independent virus adsorption on target cell membranes. Biophysical Chemistry. 2002; 97:159-172. doi:https://doi.org/10.1016/S0301-4622(02)00057-1.

126. Nanbakhsh A, Best B, Riese M, Rao S, Wang L, Medin J, et al. Dextran enhances the lentiviral transduction efficiency of murine and human primary nk cells. J Vis Exp. 2018; 131:55063. doi:10.3791/55063.

127. Johnston JM, Denning G, Moot R, Whitehead D, Shields J, Le Doux JM, et al. High-throughput screening identifies compounds that enhance lentiviral transduction. Gene Therapy. 2014; 21:1008. doi:10.1038/gt.2014.80.

128. Wang CX, Sather BD, Wang X, Adair J, Khan I, Singh S, et al. Rapamycin relieves lentiviral vector transduction resistance in human and mouse hematopoietic stem cells. Blood. 2014; 124:913-923. doi:10.1182/blood-2013-12-546218. 
129. Petrillo C, Cesana D, Piras F, Bartolaccini S, Naldini L, Montini E, et al. Cyclosporin a and rapamycin relieve distinct lentiviral restriction blocks in hematopoietic stem and progenitor cells. Mol Ther. 2015; 23:352-362. doi:https://doi.org/10.1038/mt.2014.193.

130. Anastasov N, Höfig I, Mall S, Krackhardt AM, Thirion C. Optimized lentiviral transduction protocols by use of a poloxamer enhancer, spinoculation, and scfv-antibody fusions to vsv-g. Methods Mol Biol. 2016; 1448:49-61. doi:10.1007/978-1-4939-3753-0_4.

131. Heffner GC, Bonner M, Christiansen L, Pierciey FJ, Campbell D, Smurnyy Y, et al. Prostaglandin e2 increases lentiviral vector transduction efficiency of adult human hematopoietic stem and progenitor cells. Mol Ther. 2018; 26:320-328. doi:10.1016/j.ymthe.2017.09.025.

132. Radek C, Bernadin O, Drechsel K, Cordes N, Pfeifer R, Sträßer P, et al. Vectofusin-1 improves transduction of primary human cells with diverse retroviral and lentiviral pseudotypes, enabling robust, automated closed-system manufacturing. Hum Gene Ther. 2019; 30:1477-1493. doi:10.1089/hum.2019.157.

133. Sutlu T, Nyström S, Gilljam M, Stellan B, Applequist SE, Alici E. Inhibition of intracellular antiviral defense mechanisms augments lentiviral transduction of human natural killer cells: Implications for gene therapy. Hum Gene Ther. 2012; 23:1090-1100. doi:10.1089/hum.2012.080.

134. Hastie E, Cataldi M, Marriott I, Grdzelishvili VZ. Understanding and altering cell tropism of vesicular stomatitis virus. Virus Research. 2013; 176:16-32. doi:https://doi.org/10.1016/j.virusres.2013.06.003.

135. Micucci F, Zingoni A, Piccoli M, Frati L, Santoni A, Galandrini R. High-efficient lentiviral vectormediated gene transfer into primary human nk cells. Exp Hematol. 2006; 34:1344-1352. doi:https://doi.org/10.1016/j.exphem.2006.06.001.

136. Tijani M, Munis AM, Perry C, Sanber K, Ferraresso M, Mukhopadhyay T, et al. Lentivector producer cell lines with stably expressed vesiculovirus envelopes. Mol Ther Methods Clin Dev. 2018; 10:303312. doi:https://doi.org/10.1016/j.omtm.2018.07.013.

137. Imai C, Iwamoto S, Campana D. Genetic modification of primary natural killer cells overcomes inhibitory signals and induces specific killing of leukemic cells. Blood. 2005; 106:376-383. doi:10.1182/blood-2004-12-4797.

138. De Sanctis JB, Blanca I, Radzioch D, Bianco NE. Expression and function of low-density lipoprotein receptors in cd3-cd16+cd56+ cells: Effect of interleukin 2. Cell Immunol. 1996; 167:18-29. doi:10.1006/cimm.1996.0003.

139. Streltsova MA, Barsov E, Erokhina SA, Kovalenko El. Retroviral gene transfer into primary human nk cells activated by il-2 and k562 feeder cells expressing membrane-bound il-21. J Immunol Methods. 2017; 450:90-94. doi:https://doi.org/10.1016/j.jim.2017.08.003.

140. Luni C, Michielin F, Barzon L, Calabrò V, Elvassore N. Stochastic model-assisted development of efficient low-dose viral transduction in microfluidics. Biophysical journal. 2013; 104:934-942. doi:10.1016/j.bpj.2012.12.049.

141. Tran R, Myers DR, Denning G, Shields JE, Lytle AM, Alrowais H, et al. Microfluidic transduction harnesses mass transport principles to enhance gene transfer efficiency. Mol Ther. 2017; 25:23722382. doi:https://doi.org/10.1016/j.ymthe.2017.07.002.

142. Shah NN, Baird K, Delbrook CP, Fleisher TA, Kohler ME, Rampertaap S, et al. Acute gvhd in patients receiving il-15/4-1bbl activated $\mathrm{nk}$ cells following $\mathrm{t}$-cell-depleted stem cell transplantation. Blood. 2015; 125:784-792. doi:10.1182/blood-2014-07-592881.

143. Denman CJ, Senyukov VV, Somanchi SS, Phatarpekar PV, Kopp LM, Johnson JL, et al. Membranebound il-21 promotes sustained ex vivo proliferation of human natural killer cells. PLoS One. 2012; 7:e30264. doi:10.1371/journal.pone.0030264.

144. Fujisaki H, Kakuda H, Imai C, Mullighan CG, Campana D. Replicative potential of human natural killer cells. Br J Haematol. 2009; 145:606-613. doi:10.1111/j.1365-2141.2009.07667.x.

145. Lapteva N, Durett AG, Sun J, Rollins LA, Huye LL, Fang J, et al. Large-scale ex vivo expansion and characterization of natural killer cells for clinical applications. Cytotherapy. 2012; 14:1131-1143. doi:10.3109/14653249.2012.700767.

146. Voshol H, Dullens HF, Den Otter W, Vliegenthart JF. Human natural killer cells: A convenient purification procedure and the influence of cryopreservation on cytotoxic activity. $\mathrm{J}$ Immunol Methods. 1993; 165:21-30. doi:10.1016/0022-1759(93)90102-d.

147. Fujiwara S, Akiyama M, Yamakido M, Seyama T, Kobuke K, Hakoda M, et al. Cryopreservation of human lymphocytes for assessment of lymphocyte subsets and natural killer cytotoxicity. J Immunol Methods. 1986; 90:265-273. doi:10.1016/0022-1759(86)90084-0.

148. Shah N, Martin-Antonio B, Yang H, Ku S, Lee DA, Cooper LJ, et al. Antigen presenting cell-mediated expansion of human umbilical cord blood yields log-scale expansion of natural killer cells with antimyeloma activity. PLoS One. 2013; 8:e76781. doi:10.1371/journal.pone.0076781. 
149. Woll PS, Martin CH, Miller JS, Kaufman DS. Human embryonic stem cell-derived nk cells acquire functional receptors and cytolytic activity. J Immunol. 2005; 175:5095-5103. doi:10.4049/jimmunol.175.8.5095.

150. Woll PS, Grzywacz B, Tian X, Marcus RK, Knorr DA, Verneris MR, et al. Human embryonic stem cells differentiate into a homogeneous population of natural killer cells with potent in vivo antitumor activity. Blood. 2009; 113:6094-6101. doi:10.1182/blood-2008-06-165225.

151. Ni Z, Knorr DA, Clouser CL, Hexum MK, Southern P, Mansky LM, et al. Human pluripotent stem cells produce natural killer cells that mediate anti-hiv-1 activity by utilizing diverse cellular mechanisms. J Virol. 2011; 85:43-50. doi:10.1128/jvi.01774-10.

152. Luevano M, Madrigal A, Saudemont A. Generation of natural killer cells from hematopoietic stem cells in vitro for immunotherapy. Cell Mol Immunol. 2012; 9:310-320. doi:10.1038/cmi.2012.17.

153. Knorr DA, Ni Z, Hermanson D, Hexum MK, Bendzick L, Cooper LJ, et al. Clinical-scale derivation of natural killer cells from human pluripotent stem cells for cancer therapy. Stem Cells Transl Med. 2013; 2:274-283. doi:10.5966/sctm.2012-0084.

154. Gong JH, Maki G, Klingemann HG. Characterization of a human cell line (nk-92) with phenotypical and functional characteristics of activated natural killer cells. Leukemia. 1994; 8:652-658

155. Snyder KM, Hullsiek R, Mishra HK, Mendez DC, Li Y, Rogich A, et al. Expression of a recombinant high affinity igg fc receptor by engineered $\mathrm{nk}$ cells as a docking platform for therapeutic mabs to target cancer cells. Front Immunol. 2018; 9:2873. doi:10.3389/fimmu.2018.02873.

156. Suck G, Odendahl M, Nowakowska P, Seidl C, Wels WS, Klingemann HG, et al. Nk-92: An 'off-theshelf therapeutic' for adoptive natural killer cell-based cancer immunotherapy. Cancer Immunol Immunother. 2016; 65:485-492. doi:10.1007/s00262-015-1761-x.

157. Arai S, Meagher R, Swearingen M, Myint H, Rich E, Martinson J, et al. Infusion of the allogeneic cell line nk-92 in patients with advanced renal cell cancer or melanoma: A phase i trial. Cytotherapy. 2008; 10:625-632. doi:10.1080/14653240802301872.

158. Tonn T, Schwabe D, Klingemann HG, Becker S, Esser R, Koehl U, et al. Treatment of patients with advanced cancer with the natural killer cell line nk-92. Cytotherapy. 2013; 15:1563-1570. doi:10.1016/j.jcyt.2013.06.017.

159. Kloess S, Oberschmidt O, Dahlke J, Vu XK, Neudoerfl C, Kloos A, et al. Preclinical assessment of suitable natural killer cell sources for chimeric antigen receptor natural killer-based "off-the-shelf" acute myeloid leukemia immunotherapies. Hum Gene Ther. 2019; 30:381-401. doi:10.1089/hum.2018.247.

160. Majzner RG, Mackall CL. Clinical lessons learned from the first leg of the car t cell journey. Nat Med. 2019; 25:1341-1355. doi:10.1038/s41591-019-0564-6.

161. Lapteva N, Szmania SM, van Rhee F, Rooney CM. Clinical grade purification and expansion of natural killer cells. Crit Rev Oncog. 2014; 19:121-132. doi:10.1615/critrevoncog.2014010931.

162. Lee DA. Cellular therapy: Adoptive immunotherapy with expanded natural killer cells. Immunol Rev. 2019; 290:85-99. doi:10.1111/imr.12793.

163. Ma Z, Wang Y, Kang H, Wu X. Zoledronate increases enrichment, activation and expansion of natural killer cells from umbilical cord blood. Human Cell. 2018; 31:310-312. doi:10.1007/s13577018-0213-2.

164. Yang Y, Badeti S, Tseng HC, Ma MT, Liu T, Jiang JG, et al. Superior expansion and cytotoxicity of human primary nk and car-nk cells from various sources via enriched metabolic pathways. Mol Ther Methods Clin Dev. 2020; 18:428-445. doi:10.1016/j.omtm.2020.06.014.

165. Michen S, Frosch J, Füssel M, Schackert G, Momburg F, Temme A. Artificial feeder cells expressing ligands for killer cell immunoglobulin-like receptors and cd94/nkg2a for expansion of functional primary natural killer cells with tolerance to self. Cytotherapy. 2020. doi:10.1016/j.jcyt.2020.02.004.

166. Klöß S, Oberschmidt O, Morgan M, Dahlke J, Arseniev L, Huppert V, et al. Optimization of human nk cell manufacturing: Fully automated separation, improved ex vivo expansion using il-21 with autologous feeder cells, and generation of anti-cd123-car-expressing effector cells. Hum Gene Ther. 2017; 28:897-913. doi:10.1089/hum.2017.157.

167. Masuyama J, Murakami T, Iwamoto S, Fujita S. Ex vivo expansion of natural killer cells from human peripheral blood mononuclear cells co-stimulated with anti-cd3 and anti-cd52 monoclonal antibodies. Cytotherapy. 2016; 18:80-90. doi:10.1016/j.jcyt.2015.09.011.

168. Hosseinzadeh F, Ai J, Ebrahimi-Barough S, Seyhoun I, Hajifathali A, Muhammadnejad S, et al. Natural killer cell expansion with autologous feeder layer and anti-cd3 antibody for immune cell therapy of hepatocellular carcinoma. Asian Pacific Journal of Cancer Prevention. 2019; 20:37973803. doi:10.31557/apjcp.2019.20.12.3797.

169. Min B, Choi H, Her JH, Jung MY, Kim H-J, Jung M-Y, et al. Optimization of large-scale expansion and cryopreservation of human natural killer cells for anti-tumor therapy. Immune network. 2018; 18:e31-e31. doi:10.4110/in.2018.18.e31. 
170. Rosario M, Liu B, Kong L, Collins LI, Schneider SE, Chen X, et al. The il-15-based alt-803 complex enhances fcyriiia-triggered $\mathrm{nk}$ cell responses and in vivo clearance of b cell lymphomas. Clin Cancer Res. 2016; 22:596-608. doi:10.1158/1078-0432.ccr-15-1419.

171. Felices M, Chu S, Kodal B, Bendzick L, Ryan C, Lenvik AJ, et al. II-15 super-agonist (alt-803) enhances natural killer (nk) cell function against ovarian cancer. Gynecol Oncol. 2017; 145:453-461. doi:10.1016/j.ygyno.2017.02.028.

172. Margolin K, Morishima C, Velcheti V, Miller JS, Lee SM, Silk AW, et al. Phase i trial of alt-803, a novel recombinant il15 complex, in patients with advanced solid tumors. Clin Cancer Res. 2018; 24:5552-5561. doi:10.1158/1078-0432.CCR-18-0945.

173. Tang X, Yang L, Li Z, Nalin AP, Dai H, Xu T, et al. First-in-man clinical trial of car nk-92 cells: Safety test of cd33-car nk-92 cells in patients with relapsed and refractory acute myeloid leukemia. Am J Cancer Res. 2018; 8:1083-1089

174. Zhu H, Blum RH, Bjordahl R, Gaidarova S, Rogers P, Lee TT, et al. Pluripotent stem cell-derived nk cells with high-affinity noncleavable cd16a mediate improved antitumor activity. Blood. 2020; 135:399-410. doi:10.1182/blood.2019000621.

175. Irvine DJ, Dane EL. Enhancing cancer immunotherapy with nanomedicine. Nat Rev Immunol. 2020; 20:321-334. doi:10.1038/s41577-019-0269-6.

176. Im S, Jang D, Saravanakumar G, Lee J, Kang Y, Lee YM, et al. Harnessing the formation of natural killer-tumor cell immunological synapses for enhanced therapeutic effect in solid tumors. Adv Mater. 2020; 32:e2000020. doi:10.1002/adma.202000020.

177. Ahn YH, Ren L, Kim SM, Seo S-H, Jung C-R, Kim DS, et al. A three-dimensional hyaluronic acidbased niche enhances the therapeutic efficacy of human natural killer cell-based cancer $\begin{array}{llll}\text { immunotherapy. } & \text { Biomaterials. 247:119960. }\end{array}$ doi:https://doi.org/10.1016/j.biomaterials.2020.119960.

178. Ji T, Lang J, Ning B, Qi F, Wang H, Zhang Y, et al. Enhanced natural killer cell immunotherapy by rationally assembling fc fragments of antibodies onto tumor membranes. Adv Mater. 2019; 31:e1804395. doi:10.1002/adma.201804395.

179. Smith TT, Stephan SB, Moffett HF, McKnight LE, Ji W, Reiman D, et al. In situ programming of leukaemia-specific t cells using synthetic DNA nanocarriers. Nature Nanotechnology. 2017; 12:813820. doi:10.1038/nnano.2017.57.

180. Kim K-S, Han J-H, Park J-H, Kim H-K, Choi SH, Kim GR, et al. Multifunctional nanoparticles for genetic engineering and bioimaging of natural killer (nk) cell therapeutics. Biomaterials. 2019; 221:119418. doi:https://doi.org/10.1016/j.biomaterials.2019.119418.

181. Mhaidly R, Verhoeyen E. The future: In vivo car t cell gene therapy. Mol Ther. 2019; 27:707-709. doi:10.1016/j.ymthe.2019.03.012.

182. Pfeiffer A, Thalheimer FB, Hartmann S, Frank AM, Bender RR, Danisch S, et al. In vivo generation of human cd19-car $t$ cells results in b-cell depletion and signs of cytokine release syndrome. EMBO Mol Med. 2018; 10:e9158. doi:10.15252/emmm.201809158.

183. Lee DW, Kochenderfer JN, Stetler-Stevenson M, Cui YK, Delbrook C, Feldman SA, et al. T cells expressing cd19 chimeric antigen receptors for acute lymphoblastic leukaemia in children and young adults: A phase 1 dose-escalation trial. Lancet. 2015; 385:517-528. doi:https://doi.org/10.1016/S0140-6736(14)61403-3.

184. Brudno JN, Lam N, Vanasse D, Shen Y-w, Rose JJ, Rossi J, et al. Safety and feasibility of anti-cd19 car $t$ cells with fully human binding domains in patients with b-cell lymphoma. Nat Med. 2020; 26:270280. doi:10.1038/s41591-019-0737-3.

185. Yilmaz A, Cui H, Caligiuri MA, Yu J. Chimeric antigen receptor-engineered natural killer cells for cancer immunotherapy. J Hematol Oncol. 2020; 13:168. doi:10.1186/s13045-020-00998-9.

186. Yu S, Yi M, Qin S, Wu K. Next generation chimeric antigen receptor t cells: Safety strategies to overcome toxicity. Molecular Cancer. 2019; 18:125. doi:10.1186/s12943-019-1057-4.

187. Ruella M, Barrett DM, Shestova O, Perazzelli J, Posey AD, Jr., Hong SJ, et al. A cellular antidote to specifically deplete anti-cd19 chimeric antigen receptor-positive cells. Blood. 2020; 135:505-509. doi:10.1182/blood.2019001859.

188. Wang X, Chang W-C, Wong CW, Colcher D, Sherman M, Ostberg JR, et al. A transgene-encoded cell surface polypeptide for selection, in vivo tracking, and ablation of engineered cells. Blood. 2011; 118:1255-1263. doi:10.1182/blood-2011-02-337360.

189. Xie G, Dong H, Liang Y, Ham JD, Rizwan R, Chen J. Car-nk cells: A promising cellular $\begin{array}{llll}\text { immunotherapy for } & \text { cancer. } & \text { EBioMedicine. } & \text { 2020; }\end{array}$ doi:https://doi.org/10.1016/j.ebiom.2020.102975.

190. Ueda T, Kumagai A, Iriguchi S, Yasui Y, Miyasaka T, Nakagoshi K, et al. Non-clinical efficacy, safety and stable clinical cell processing of induced pluripotent stem cell-derived anti-glypican-3 chimeric 
antigen receptor-expressing natural killer/innate lymphoid cells. Cancer Sci. 2020; 111:1478-1490. doi:10.1111/cas.14374.

191. Ingegnere T, Mariotti FR, Pelosi A, Quintarelli C, De Angelis B, Tumino N, et al. Human car nk cells: A new non-viral method allowing high efficient transfection and strong tumor cell killing. Front Immunol. 2019; 10:957. doi:10.3389/fimmu.2019.00957.

192. Piras F, Riba M, Petrillo C, Lazarevic D, Cuccovillo I, Bartolaccini S, et al. Lentiviral vectors escape innate sensing but trigger p53 in human hematopoietic stem and progenitor cells. EMBO Mol Med. 2017; 9:1198-1211. doi:10.15252/emmm.201707922.

193. Ruella M, Xu J, Barrett DM, Fraietta JA, Reich TJ, Ambrose DE, et al. Induction of resistance to chimeric antigen receptor $t$ cell therapy by transduction of a single leukemic b cell. Nat Med. 2018; 24:1499-1503. doi:10.1038/s41591-018-0201-9.

194. Fraietta JA, Nobles CL, Sammons MA, Lundh S, Carty SA, Reich TJ, et al. Disruption of tet2 promotes the therapeutic efficacy of cd19-targeted $t$ cells. Nature. 2018; 558:307-312. doi:10.1038/s41586-018-0178-z.

195. Howe SJ, Mansour MR, Schwarzwaelder K, Bartholomae C, Hubank M, Kempski H, et al. Insertional mutagenesis combined with acquired somatic mutations causes leukemogenesis following gene therapy of scid-x1 patients. J Clin Invest. 2008; 118:3143-3150. doi:10.1172/JCI35798.

196. Hacein-Bey-Abina S, Von Kalle C, Schmidt M, McCormack MP, Wulffraat N, Leboulch P, et al. Lmo2-associated clonal t cell proliferation in two patients after gene therapy for scid-x1. Science. 2003; 302:415-419. doi:10.1126/science.1088547.

197. Marcucci KT, Jadlowsky JK, Hwang W-T, Suhoski-Davis M, Gonzalez VE, Kulikovskaya I, et al. Retroviral and lentiviral safety analysis of gene-modified t cell products and infused hiv and oncology patients. Mol Ther. 2018; 26:269-279. doi:10.1016/j.ymthe.2017.10.012.

198. Haapaniemi E, Botla S, Persson J, Schmierer B, Taipale J. Crispr-cas9 genome editing induces a p53-mediated DNA damage response. Nat Med. 2018; 24:927-930. doi:10.1038/s41591-018-0049$z$.

199. Charlesworth CT, Deshpande PS, Dever DP, Camarena J, Lemgart VT, Cromer MK, et al. Identification of preexisting adaptive immunity to cas9 proteins in humans. Nat Med. 2019; 25:249254. doi:10.1038/s41591-018-0326-x.

200. Li R, Johnson R, Yu G, McKenna DH, Hubel A. Preservation of cell-based immunotherapies for clinical trials. Cytotherapy. 2019; 21:943-957. doi:https://doi.org/10.1016/j.jcyt.2019.07.004.

201. Yao X, Jovevski JJ, Todd MF, Xu R, Li Y, Wang J, et al. Nanoparticle-mediated intracellular protection of natural killer cells avoids cryoinjury and retains potent antitumor functions. Adv Sci. 2020; 7:1902938. doi:10.1002/advs.201902938.

202. Rodriguez-Garcia A, Palazon A, Noguera-Ortega E, Powell DJ, Jr., Guedan S. Car-t cells hit the tumor microenvironment: Strategies to overcome tumor escape. Front Immunol. 2020; 11:1109. doi:10.3389/fimmu.2020.01109.

203. Cherkassky L, Morello A, Villena-Vargas J, Feng Y, Dimitrov DS, Jones DR, et al. Human car t cells with cell-intrinsic pd-1 checkpoint blockade resist tumor-mediated inhibition. J Clin Invest. 2016; 126:3130-3144. doi:10.1172/jci83092.

204. Heczey A, Louis CU, Savoldo B, Dakhova O, Durett A, Grilley B, et al. Car t cells administered in combination with lymphodepletion and pd-1 inhibition to patients with neuroblastoma. Mol Ther. 2017; 25:2214-2224. doi:10.1016/j.ymthe.2017.05.012.

205. Tang N, Cheng C, Zhang X, Qiao M, Li N, Mu W, et al. Tgf- $\beta$ inhibition via crispr promotes the longterm efficacy of car $t$ cells against solid tumors. JCl Insight. 2020; 5:e133977. doi:10.1172/jci.insight.133977.

206. Kloss CC, Lee J, Zhang A, Chen F, Melenhorst JJ, Lacey SF, et al. Dominant-negative tgf- $\beta$ receptor enhances psma-targeted human car $\mathrm{t}$ cell proliferation and augments prostate cancer eradication. Mol Ther. 2018; 26:1855-1866. doi:10.1016/j.ymthe.2018.05.003.

207. Juillerat A, Marechal A, Filhol JM, Valogne Y, Valton J, Duclert A, et al. An oxygen sensitive selfdecision making engineered car t-cell. Sci Rep. 2017; 7:39833. doi:10.1038/srep39833.

208. Buchan SL, Dou L, Remer M, Booth SG, Dunn SN, Lai C, et al. Antibodies to costimulatory receptor 4-1 bb enhance anti-tumor immunity via t regulatory cell depletion and promotion of cd8 $\mathrm{t}$ cell effector function. Immunity. 2018; 49:958-970.e957. doi:10.1016/j.immuni.2018.09.014.

209. Parihar R, Rivas C, Huynh M, Omer B, Lapteva N, Metelitsa LS, et al. Nk cells expressing a chimeric activating receptor eliminate mdscs and rescue impaired car-t cell activity against solid tumors. Cancer Immunol Res. 2019; 7:363-375. doi:10.1158/2326-6066.cir-18-0572.

210. Ruella M, Klichinsky M, Kenderian SS, Shestova O, Ziober A, Kraft DO, et al. Overcoming the immunosuppressive tumor microenvironment of hodgkin lymphoma using chimeric antigen receptor t cells. Cancer Discov. 2017; 7:1154-1167. doi:10.1158/2159-8290.cd-16-0850. 
211. Jo Y, Ali LA, Shim JA, Lee BH, Hong C. Innovative car-t cell therapy for solid tumor; current duel between car-t spear and tumor shield. Cancers (Basel). 2020; 12. doi:10.3390/cancers12082087.

212. Koneru M, Purdon TJ, Spriggs D, Koneru S, Brentjens RJ. II-12 secreting tumor-targeted chimeric antigen receptor t cells eradicate ovarian tumors in vivo. Oncoimmunology. 2015; 4:e994446. doi:10.4161/2162402x.2014.994446.

213. Pegram HJ, Lee JC, Hayman EG, Imperato GH, Tedder TF, Sadelain M, et al. Tumor-targeted $t$ cells modified to secrete il-12 eradicate systemic tumors without need for prior conditioning. Blood. 2012; 119:4133-4141. doi:10.1182/blood-2011-12-400044.

214. Krenciute G, Prinzing BL, Yi Z, Wu MF, Liu H, Dotti G, et al. Transgenic expression of il15 improves antiglioma activity of il13ra2-car t cells but results in antigen loss variants. Cancer Immunol Res. 2017; 5:571-581. doi:10.1158/2326-6066.cir-16-0376.

215. Avanzi MP, Yeku O, Li X, Wijewarnasuriya DP, van Leeuwen DG, Cheung K, et al. Engineered tumor-targeted $t$ cells mediate enhanced anti-tumor efficacy both directly and through activation of the endogenous immune system. Cell Rep. 2018; 23:2130-2141. doi:10.1016/j.celrep.2018.04.051.

216. Hu B, Ren J, Luo Y, Keith B, Young RM, Scholler J, et al. Augmentation of antitumor immunity by human and mouse car $t$ cells secreting il-18. Cell Rep. 2017; 20:3025-3033. doi:10.1016/j.celrep.2017.09.002.

217. Kagoya Y, Tanaka S, Guo T, Anczurowski M, Wang CH, Saso K, et al. A novel chimeric antigen receptor containing a jak-stat signaling domain mediates superior antitumor effects. Nat Med. 2018; 24:352-359. doi:10.1038/nm.4478.

218. Shum T, Omer B, Tashiro H, Kruse RL, Wagner DL, Parikh K, et al. Constitutive signaling from an engineered il7 receptor promotes durable tumor elimination by tumor-redirected $\mathrm{t}$ cells. Cancer Discov. 2017; 7:1238-1247. doi:10.1158/2159-8290.cd-17-0538.

219. Brown CE, Vishwanath RP, Aguilar B, Starr R, Najbauer J, Aboody KS, et al. Tumor-derived chemokine mcp-1/ccl2 is sufficient for mediating tumor tropism of adoptively transferred $t$ cells. J Immunol. 2007; 179:3332-3341. doi:10.4049/jimmunol.179.5.3332.

220. Craddock JA, Lu A, Bear A, Pule M, Brenner MK, Rooney CM, et al. Enhanced tumor trafficking of $\mathrm{gd} 2$ chimeric antigen receptor $\mathrm{t}$ cells by expression of the chemokine receptor ccr $2 \mathrm{~b}$. J Immunother. 2010; 33:780-788. doi:10.1097/CJI.0b013e3181ee6675.

221. Moon EK, Carpenito C, Sun J, Wang LC, Kapoor V, Predina J, et al. Expression of a functional ccr2 receptor enhances tumor localization and tumor eradication by retargeted human t cells expressing a mesothelin-specific chimeric antibody receptor. Clin Cancer Res. 2011; 17:4719-4730. doi:10.1158/1078-0432.ccr-11-0351.

222. Rapp M, Grassmann S, Chaloupka M, Layritz P, Kruger S, Ormanns S, et al. C-c chemokine receptor type-4 transduction of $\mathrm{t}$ cells enhances interaction with dendritic cells, tumor infiltration and therapeutic efficacy of adoptive $t$ cell transfer. Oncoimmunology. 2016; 5:e1105428. doi:10.1080/2162402x.2015.1105428.

223. Di Stasi A, De Angelis B, Rooney CM, Zhang L, Mahendravada A, Foster AE, et al. T lymphocytes coexpressing ccr4 and a chimeric antigen receptor targeting cd30 have improved homing and antitumor activity in a hodgkin tumor model. Blood. 2009; 113:6392-6402. doi:10.1182/blood-200903-209650.

224. Carlsten M, Levy E, Karambelkar A, Li L, Reger R, Berg M, et al. Efficient mrna-based genetic engineering of human nk cells with high-affinity cd16 and ccr7 augments rituximab-induced adcc against lymphoma and targets nk cell migration toward the lymph node-associated chemokine ccl19. Front Immunol. 2016; 7:105. doi:10.3389/fimmu.2016.00105.

225. Kershaw MH, Wang G, Westwood JA, Pachynski RK, Tiffany HL, Marincola FM, et al. Redirecting migration of $\mathrm{t}$ cells to chemokine secreted from tumors by genetic modification with cxcr2. Hum Gene Ther. 2002; 13:1971-1980. doi:10.1089/10430340260355374.

226. Hillerdal V, Essand M. Chimeric antigen receptor-engineered t cells for the treatment of metastatic prostate cancer. BioDrugs. 2015; 29:75-89. doi:10.1007/s40259-015-0122-9.

227. Chen KH, Wada M, Pinz KG, Liu H, Shuai X, Chen X, et al. A compound chimeric antigen receptor strategy for targeting multiple myeloma. Leukemia. 2018; 32:402-412. doi:10.1038/leu.2017.302.

228. Kloss CC, Condomines M, Cartellieri M, Bachmann M, Sadelain M. Combinatorial antigen recognition with balanced signaling promotes selective tumor eradication by engineered t cells. Nat Biotechnol. 2013; 31:71-75. doi:10.1038/nbt.2459.

229. Hegde M, Mukherjee M, Grada Z, Pignata A, Landi D, Navai SA, et al. Tandem car t cells targeting her2 and il13ra2 mitigate tumor antigen escape. J Clin Invest. 2016; 126:3036-3052. doi:10.1172/jci83416.

230. Bielamowicz K, Fousek K, Byrd TT, Samaha H, Mukherjee M, Aware N, et al. Trivalent car t cells overcome interpatient antigenic variability in glioblastoma. Neuro Oncol. 2018; 20:506-518. doi:10.1093/neuonc/nox182. 
231. Caratelli S, Sconocchia T, Arriga R, Coppola A, Lanzilli G, Lauro D, et al. Fcy chimeric receptorengineered t cells: Methodology, advantages, limitations, and clinical relevance. Front Immunol. 2017; 8:457. doi:10.3389/fimmu.2017.00457.

232. Tamada K, Geng D, Sakoda Y, Bansal N, Srivastava R, Li Z, et al. Redirecting gene-modified t cells toward various cancer types using tagged antibodies. Clin Cancer Res. 2012; 18:6436-6445. doi:10.1158/1078-0432.ccr-12-1449.

233. Feldmann A, Arndt C, Bergmann R, Loff S, Cartellieri M, Bachmann D, et al. Retargeting of $t$ lymphocytes to psca- or psma positive prostate cancer cells using the novel modular chimeric antigen receptor platform technology "unicar". Oncotarget. 2017; 8:31368-31385. doi:10.18632/oncotarget.15572.

234. Fedorov VD, Themeli M, Sadelain M. Pd-1- and ctla-4-based inhibitory chimeric antigen receptors (icars) divert off-target immunotherapy responses. Sci Transl Med. 2013; 5:215ra172. doi:10.1126/scitranslmed.3006597.

235. Morsut L, Roybal KT, Xiong X, Gordley RM, Coyle SM, Thomson M, et al. Engineering customized cell sensing and response behaviors using synthetic notch receptors. Cell. 2016; 164:780-791. doi:10.1016/j.cell.2016.01.012.

236. Mata M, Gerken C, Nguyen P, Krenciute G, Spencer DM, Gottschalk S. Inducible activation of myd88 and cd40 in car t cells results in controllable and potent antitumor activity in preclinical solid tumor models. Cancer Discov. 2017; 7:1306-1319. doi:10.1158/2159-8290.cd-17-0263.

237. Rupp LJ, Schumann K, Roybal KT, Gate RE, Ye CJ, Lim WA, et al. Crispr/cas9-mediated pd-1 disruption enhances anti-tumor efficacy of human chimeric antigen receptor t cells. Sci Rep. 2017; 7:737. doi:10.1038/s41598-017-00462-8.

238. Ren J, Liu X, Fang C, Jiang S, June $\mathrm{CH}$, Zhao Y. Multiplex genome editing to generate universal car $t$ cells resistant to pd1 inhibition. Clin Cancer Res. 2017; 23:2255-2266. doi:10.1158/10780432.ccr-16-1300. 
Table 1. Overview of CAR-NK studies to date using cell lines.

\begin{tabular}{|c|c|c|c|c|c|c|c|c|c|c|c|c|}
\hline \multirow[b]{2}{*}{ \# } & \multirow{2}{*}{$\begin{array}{l}\text { Reference } \\
\text { First author (journal, } \\
\text { year) }\end{array}$} & \multirow[b]{2}{*}{$\begin{array}{l}\text { Effector } \\
\text { cell }\end{array}$} & \multicolumn{5}{|c|}{ Vehicle } & \multirow[b]{2}{*}{ Target } & \multicolumn{3}{|c|}{ gen. } & \multirow[b]{2}{*}{$\begin{array}{l}\text { Activation } \\
\text { signal }\end{array}$} \\
\hline & & & 온 & $\underline{\underline{\underline{E}}}$ & $\begin{array}{l}\underset{\nwarrow}{\Sigma} \\
\substack{\alpha \\
\varepsilon} \\
\end{array}$ & $\begin{array}{l}\frac{\overline{0}}{E} \\
\frac{1}{0} \\
\frac{\pi}{\alpha} \\
\end{array}$ & 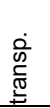 & & 1 & 2 & 3 & \\
\hline 1 & Uherek (Blood 2002) & NK-92 & $x$ & & & & & HER2 & $x$ & & & $\mathrm{CD} 3 \zeta$ \\
\hline 2 & $\begin{array}{l}\text { Schirrmann (Cancer } \\
\text { Gene Ther 2002) }\end{array}$ & YTS & & & & $x$ & & CEA & $x$ & & & $\mathrm{CD} 3 \zeta$ \\
\hline 3 & $\begin{array}{ll}\text { Daldrup-Link } & \text { (Eur } \\
\text { Radiol 2005) } & \\
\end{array}$ & NK-92 & $x$ & $x$ & & & & HER2 & $\mathrm{x}$ & & & $\mathrm{CD} 3 \zeta$ \\
\hline 4 & $\begin{array}{l}\text { Schirrmann (Leukemia } \\
\text { Research 2005) }\end{array}$ & $\mathrm{YT}$ & & & & $\mathrm{x}$ & & CD33 & $x$ & & & $\mathrm{CD} 3 \zeta$ \\
\hline 5 & $\begin{array}{l}\text { Meier (Nucl Med Biol } \\
2008 \text { ) }\end{array}$ & NK-92 & $x$ & & & & & HER2 & $\mathrm{x}$ & & & $\mathrm{CD} 3 \zeta$ \\
\hline 6 & $\begin{array}{l}\text { Muller (Cancer Immunol } \\
\text { Immunother 2008) }\end{array}$ & NK-92 & $x$ & & & & & CD20 & $x$ & & & murine $\mathrm{CD} 3 \zeta$ \\
\hline 7 & $\begin{array}{lll}\text { Tavri } & \text { (Mol } & \text { Imaging } \\
2009) & & \\
\end{array}$ & NK-92 & $x$ & & & & & EpCAM & $x$ & & & $\mathrm{CD} 3 \zeta$ \\
\hline 8 & $\begin{array}{l}\text { Boissel (Leukemia Res } \\
\text { 2009) }\end{array}$ & NK-92 & & & $x$ & $x$ & & CD19 & $\mathrm{x}$ & & & $\mathrm{CD} 3 \zeta$ \\
\hline 9 & $\begin{array}{l}\text { Meier (Magn Reson } \\
\text { Med 2011) }\end{array}$ & NK-92 & $x$ & & & & & EpCAM & $x$ & & & $\mathrm{CD} 3 \zeta$ \\
\hline 10 & $\begin{array}{l}\text { Sahm (Cancer Immunol } \\
\text { Immunother 2012) }\end{array}$ & $\begin{array}{l}\text { NK-92, } \\
\text { NKL }\end{array}$ & & 2 & & & & EpCAM & & $x$ & & CD28-CD3ろ \\
\hline 11 & $\begin{array}{l}\text { Boissel (Leukemia } \\
\text { Lymphoma 2012) }\end{array}$ & NK-92 & & $\mathrm{x}$ & $x$ & & & CD19, CD20 & $x$ & & & $\mathrm{CD} 3 \zeta$ \\
\hline 12 & $\begin{array}{l}\text { Esser (J Cell Mol Med } \\
\text { 2012) }\end{array}$ & NK-92 & $x$ & & & & & GD2 & $\mathrm{x}$ & & & $\mathrm{CD} 3 \zeta$ \\
\hline 13 & $\begin{array}{l}\text { Tassev (Cancer Gene } \\
\text { Ther 2012) }\end{array}$ & NK-92-MI & $x$ & & & & & EBNA & $x$ & & & $\mathrm{CD} 3 \zeta$ \\
\hline 14 & $\begin{array}{lll}\text { Alkins } & \text { (Cancer } & \text { Res } \\
2013) & & \\
\end{array}$ & NK-92 & $x$ & & & & & HER2 & $x$ & & & CD3ろ \\
\hline 15 & $\begin{array}{l}\text { Boissel (Oncolmmunol } \\
2014 \text { ) }\end{array}$ & NK-92 & & $x$ & & & & CD19, CD20 & $x$ & & & $\mathrm{CD} 3 \zeta$ \\
\hline 16 & Jiang (Mol Oncol 2014) & NK-92-MI & & 3 & & & & CD138 & $x$ & & & $\mathrm{CD} 3 \zeta$ \\
\hline 17 & Chu (Leukemia 2014) & NK-92 & & 2 & & & & CS1 & & $x$ & & CD28-CD3ろ \\
\hline 18 & $\begin{array}{l}\text { Seidel (Cancer Immunol } \\
\text { Immunother 2015) }\end{array}$ & NK-92 & $x$ & & & & & GD2 & $x$ & & & $\mathrm{CD} 3 \zeta$ \\
\hline 19 & $\begin{array}{l}\text { Schönfeld (Mol Ther } \\
\text { 2015) }\end{array}$ & NK-92 & & 2 & & & & HER2 & $x$ & $x$ & & 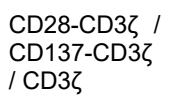 \\
\hline 20 & Han (Sci Reports 2015) & $\begin{array}{l}\text { NK-92, } \\
\text { NKL }\end{array}$ & & 2 & & & & EGFR & & $x$ & & CD28-CD3ろ \\
\hline 21 & $\begin{array}{l}\text { Clémenceau (J Immunol } \\
\text { Res 2015) }\end{array}$ & NK-92 & $x$ & & & & & Her2 & $x$ & & & FceRl $\gamma$ \\
\hline 22 & $\begin{array}{ll}\text { Liu (Oncol Reports } \\
\text { 2015) }\end{array}$ & NK-92 & & & & $x$ & & HER2 & & $x$ & & CD28-CD3ろ \\
\hline 23 & Zhao (Leukemia 2015) & NK-92-MI & $x$ & & & & & WT-1 & & $x$ & & CD137-CD3ろ \\
\hline
\end{tabular}


Chapter 4 | 133

\begin{tabular}{|c|c|c|c|c|c|c|c|c|c|c|}
\hline 24 & $\begin{array}{l}\text { Muller (J Immunother } \\
\text { 2015) }\end{array}$ & YTS & & 2 & & EGFRvIII & $\mathrm{x}$ & & & DAP12 \\
\hline 25 & $\begin{array}{ll}\text { Alkins } & \text { (Neuro-Oncol } \\
2016) & \end{array}$ & NK-92 & $x$ & & & HER2 & $\mathrm{x}$ & & & $\mathrm{CD} 3 \zeta$ \\
\hline 26 & $\begin{array}{l}\text { Genßler et al. } \\
\text { (Oncolmmunol 2016) }\end{array}$ & NK-92 & & 2 & & EGFRvIII & & $x$ & & CD28-CD3ろ \\
\hline 27 & $\begin{array}{l}\text { Zhang (J Natl Cancer } \\
\text { Inst 2016) }\end{array}$ & NK-92 & & 2 & & HER2 & & $\mathrm{x}$ & & CD28-CD3ろ \\
\hline 28 & $\begin{array}{l}\text { Romanski (J Cell Mol } \\
\text { Med 2016) }\end{array}$ & NK-92 & $x$ & & & CD19 & $\mathrm{x}$ & & & $\mathrm{CD} 3 \zeta$ \\
\hline 29 & $\begin{array}{l}\text { Topfer (J Immunother } \\
\text { 2016) }\end{array}$ & YTS & & 2 & & EGFRvIII & $x$ & & & DAP12 \\
\hline 30 & Chen (Oncotarget 2016) & $\begin{array}{l}\text { NK-92, } \\
\text { PB-NK }\end{array}$ & & 2 & & EGFR & & $\mathrm{x}$ & & CD28-CD3ろ \\
\hline 31 & Siegler (Mol Ther 2017) & NK-92 & $x$ & $x$ & & CD19, HER2 & & $x$ & $x$ & 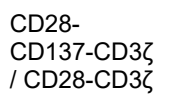 \\
\hline 32 & $\begin{array}{l}\text { Oelsner (Cytotherapy } \\
\text { 2017) }\end{array}$ & NK-92 & & 2 & & CD19 & $x$ & $x$ & & 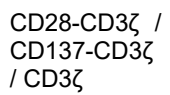 \\
\hline 33 & Chen (Oncotarget 2017) & NK-92 & & 2 & & CD3 & & & $x$ & $\begin{array}{l}\text { CD137- } \\
\text { CD28-CD3ろ }\end{array}$ \\
\hline 34 & Chen (Leukemia 2017) & NK-92 & & 2 & & CD5 & & & $x$ & $\begin{array}{l}\text { CD137- } \\
\text { CD28-CD3ろ }\end{array}$ \\
\hline 35 & Li (Cell Stem Cell 2018) & $\begin{array}{l}\text { NK-92, } \\
\text { iPSC }\end{array}$ & & & $x$ & mesothelin & $x$ & $x$ & $x$ & 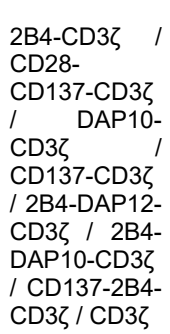 \\
\hline 36 & $\begin{array}{l}\text { Zhao (Eur J Inflamm } \\
\text { 2018) }\end{array}$ & NK-92-MI & & $x$ & & $\begin{array}{l}\text { CD19, } \\
\text { CD138 }\end{array}$ & & $x$ & & CD28-CD3 $\zeta$ \\
\hline 37 & $\begin{array}{ll}\text { Murakami } & \text { (Anticancer } \\
\text { Res 2018) } & \end{array}$ & KHYG-1 & & $x$ & & EGFRvIII & & $x$ & & CD137-CD3ろ \\
\hline 38 & $\begin{array}{l}\text { Zhang (J Immunol Res } \\
\text { 2018) }\end{array}$ & NK-92 & & 2 & & EpCAM & & $x$ & & CD137-CD3ろ \\
\hline 39 & Yu (Mol Therapy 2018) & NK-92 & & $x$ & & GPC3 & & $x$ & & CD28-CD3ろ \\
\hline 40 & $\begin{array}{l}\text { Nowakowska (Cancer } \\
\text { Immunol Immunother } \\
\text { 2018) }\end{array}$ & NK-92 & & 2 & & HER2 & $x$ & $x$ & & 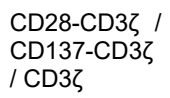 \\
\hline 41 & $\begin{array}{l}\text { Tang (Am J Cancer Res } \\
\text { 2018) }\end{array}$ & NK-92-MI & & 3 & & CD33 & $x$ & $x$ & & $\begin{array}{l}\text { CD28- } \\
\text { CD137-CD3ろ }\end{array}$ \\
\hline 42 & $\begin{array}{l}\text { Schnalzger }(\text { EMBO J } \\
2019)\end{array}$ & NK-92 & & 2 & & HER2 & & $x$ & & CD28-CD3ろ \\
\hline
\end{tabular}


134 | Chapter 4

\begin{tabular}{|c|c|c|c|c|c|c|c|c|c|c|c|c|}
\hline 43 & Ao (J Immunother 2019) & NK-92 & & $x$ & & & & AlphaFR & $x$ & $x$ & $x$ & 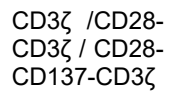 \\
\hline 44 & $\begin{array}{l}\text { Kloess (Human Gene } \\
\text { Therapy 2019) }\end{array}$ & $\begin{array}{l}\text { NK-92, } \\
\text { PB }\end{array}$ & $x$ & & & & & CD123 & & & $x$ & $\begin{array}{l}\text { CD28- } \\
\text { CD137-CD3ろ }\end{array}$ \\
\hline 45 & Kim (Biomaterials 2019) & NK-92-MI & & & & $x$ & & EGFR & & $x$ & & CD28-CD3ろ \\
\hline 46 & $\begin{array}{l}\text { Xu (J Hematol Oncol } \\
2019)\end{array}$ & NK-92 & & 3 & & & & CD5 & & $x$ & & 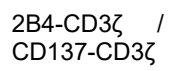 \\
\hline 47 & Batchu (Surgery 2019) & NK-92-MI & & & & $x$ & $x$ & mesothelin & & & $x$ & $\begin{array}{l}\text { CD28- } \\
\text { CD137-CD3ろ }\end{array}$ \\
\hline 48 & $\begin{array}{l}\text { Kulemzin (BMC Med } \\
\text { Genom 2019) }\end{array}$ & $\begin{array}{l}\text { NK-92, } \\
\text { YT }\end{array}$ & & 2 & & & & PMSA & & $x$ & & CD28-CD3ろ \\
\hline 49 & $\begin{array}{l}\text { You (Am J Cancer Res. } \\
\text { 2019) }\end{array}$ & NK-92-MI & & & & & $x$ & $\mathrm{CD} 7$ & & & $x$ & $\begin{array}{l}\text { CD28-4-1BB- } \\
\text { CD3Z }\end{array}$ \\
\hline 50 & $\begin{array}{l}\text { Oelsner (Int J Cancer } \\
\text { 2019) }\end{array}$ & NK-92 & & 2 & & & & FLT3 & & $x$ & & CD28-CD3ろ \\
\hline 51 & $\begin{array}{l}\text { Parlar (Eur J Immunol } \\
\text { 2019) }\end{array}$ & $\begin{array}{l}\text { NK-92, } \\
\text { YTS }\end{array}$ & & $x$ & & & & 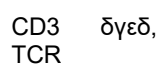 & $x$ & & & $\mathrm{CD} 3 \zeta$ \\
\hline 52 & $\begin{array}{l}\text { Mensali } \quad \text { (EBioMed } \\
2019)\end{array}$ & NK-92 & $x$ & & & & & $\begin{array}{l}\text { CD3 ठүघठ, } \\
\text { TCR }\end{array}$ & $x$ & & & $\mathrm{CD} 3 \zeta$ \\
\hline 53 & Ravi (Leukemia 2020) & NK-92 & & $x$ & & & & CD19 & $x$ & & & $\mathrm{CD} 3 \zeta$ \\
\hline 54 & Hu (Sci Report 2020) & NK-92-MI & & $x$ & & & & Tissue factor & & $x$ & & CD137-CD3ろ \\
\hline 55 & $\begin{array}{l}\text { Huang (Cancer Manag } \\
\text { Res 2020) }\end{array}$ & NK-92 & & $x$ & & & & GPC3 & $x$ & $x$ & $x$ & 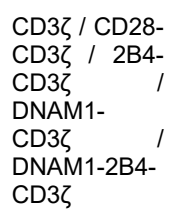 \\
\hline 56 & $\begin{array}{lll}\begin{array}{l}\text { Mitwasi } \\
\text { 2020) }\end{array} & \text { (Sci } & \text { Report } \\
\end{array}$ & NK-92-MI & & $x$ & & & & GD2 & & $x$ & & CD28-CD3ろ \\
\hline 57 & Hambach (Cells 2020) & NK-92 & $x$ & & & & & CD38 & & & $x$ & $\begin{array}{l}\text { CD28- } \\
\text { CD137-CD3ろ }\end{array}$ \\
\hline 58 & $\begin{array}{l}\text { Cao (Biochem Biophys } \\
\text { Res Commun 2020) }\end{array}$ & NK-92 & & 2 & & & & $\begin{array}{l}\text { Mesothelin, } \\
\text { CD19 }\end{array}$ & & & $x$ & $\begin{array}{l}\text { CD28- } \\
\text { CD137-CD3ろ }\end{array}$ \\
\hline 59 & $\begin{array}{l}\text { Li (J Cancer Res Ther } \\
2020)\end{array}$ & NK-92 & & $x$ & & & & Robo1 & & $x$ & & CD137-CD3ろ \\
\hline 60 & Montagner (Cells 2020) & NK-92 & & $x$ & & & & PMSA & & $x$ & & $\mathrm{CD} 28-\mathrm{CD} 3 \zeta$ \\
\hline 61 & $\begin{array}{ll}\text { Nakazawa } & \text { (Anticancer } \\
\text { Res 2020) } & \end{array}$ & KHYG-1 & & $x$ & & & & EGFRvIII & & $x$ & & CD137-CD3ろ \\
\hline 62 & Robbins (eLife 2020) & NK-92 & - & - & - & - & - & PDL1 & $x$ & & & FceR1y \\
\hline 63 & $\begin{array}{l}\text { Lee (J Control Release } \\
2020)\end{array}$ & NK-92 & & $x$ & & & & Fra, TRAIL & & $x$ & & CD27-CD3ろ \\
\hline 64 & Liu (Cytotherapy 2020) & NK-92 MI & & 3 & & & & CD19 & & $x$ & & CD137-CD3ろ \\
\hline 65 & $\begin{array}{l}\text { Yang (Front Pharmacol } \\
2020 \text { ) }\end{array}$ & NK-92 Ml & & 2 & & & & $\mathrm{~B} 7 \mathrm{H} 3$ & & $x$ & & CD137-CD3ろ \\
\hline
\end{tabular}




\begin{tabular}{|c|c|c|c|c|c|c|c|c|c|c|c|c|}
\hline 66 & $\begin{array}{l}\text { Jamali (Front immunol } \\
\text { 2020) }\end{array}$ & $\begin{array}{l}\text { NK-92, } \\
\text { NKL, PB }\end{array}$ & & 3 & & & & CD19 & & $x$ & & CD137-CD3ろ \\
\hline 67 & Ahn (Biomaterials 2020) & NK-92 & - & - & - & - & - & EGFR & & & $x$ & $\begin{array}{l}\text { CD28- } \\
\text { DAP10-CD3ろ }\end{array}$ \\
\hline 68 & $\begin{array}{l}\text { Fabian (J Immunother } \\
\text { Cancer 2020) }\end{array}$ & haNK-92 & - & - & - & - & - & PD-L1 & - & - & - & - \\
\hline 69 & $\begin{array}{l}\text { Gurney (Haematologica } \\
\text { 2020) }\end{array}$ & $\begin{array}{l}\text { KHYG-1, } \\
\text { PB-NK }\end{array}$ & $x$ & & & & & CD38 & & $x$ & & CD28-CD3ろ \\
\hline 70 & $\begin{array}{l}\text { Tseng (Nat Commun } \\
\text { 2020) }\end{array}$ & $\begin{array}{l}\text { NK-92MI, } \\
\text { PB-NK }\end{array}$ & $x$ & 2 & & & & $\begin{array}{l}\text { CD147, } \\
\text { GPC3 }\end{array}$ & & & $x$ & $\begin{array}{l}\text { CD28- } \\
\text { CD137-CD3ろ }\end{array}$ \\
\hline 71 & $\begin{array}{lr}\text { Eitler (Journal } & \text { for } \\
\text { ImmunoTherapy } & \text { of } \\
\text { Cancer 2021) } & \end{array}$ & NK-92 & & 2 & & & & HER2 & & $x$ & & CD28-CD3ろ \\
\hline 72 & $\begin{array}{l}\text { Kang (Int J Mol Sci } \\
2021)\end{array}$ & $\begin{array}{l}\text { NK-92MI, } \\
\text { KHYG-1 }\end{array}$ & & 3 & & & & CD19 & & & & CD28-CD3ろ \\
\hline
\end{tabular}

Numbers in the "Vehicle" column refer to the generation of retro- and lentiviruses. - denotes information not provided in the study. Other abbreviations: gen. = CAR generation. Lenti = lentivirus. Retro = retrovirus. Transp = transposon.

There is an interactive and extended version of this table available online, also providing regular updates: http://www.carnkreview.com 
Table 2. Primary cell-derived CAR-NK cells.

\begin{tabular}{|c|c|c|c|c|c|c|c|c|c|c|c|c|}
\hline \multirow[b]{2}{*}{ \# } & \multirow{2}{*}{$\begin{array}{l}\text { Reference } \\
\text { First author (journal, } \\
\text { year) }\end{array}$} & \multirow[b]{2}{*}{$\begin{array}{l}\text { Effector } \\
\text { cell }\end{array}$} & \multicolumn{5}{|c|}{ Vehicle } & \multirow[b]{2}{*}{ Target } & \multicolumn{3}{|c|}{ gen. } & \multirow[b]{2}{*}{$\begin{array}{l}\text { Activation } \\
\text { signal }\end{array}$} \\
\hline & & & 올 & $\begin{array}{l}\stackrel{\vec{F}}{\underline{D}} \\
\underline{\underline{D}}\end{array}$ & 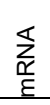 & $\begin{array}{l}\frac{\overline{0}}{\varepsilon} \\
\frac{1}{2} \\
\frac{\pi}{Q} \\
\end{array}$ & 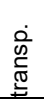 & & 1 & 2 & 3 & \\
\hline 1 & Imai (Blood 2005) & PB-NK & $x$ & & & & & CD19 & $x$ & $x$ & & 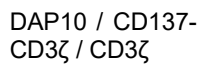 \\
\hline 2 & $\begin{array}{l}\text { Altvater (Clin Cancer } \\
\text { Res 2009) }\end{array}$ & PB-NK & $x$ & & & & & CD19, GD2 & $x$ & $x$ & & $\begin{array}{l}\mathrm{CD} 3 \zeta \text { / 2B } 2 \text { I } \\
2 \mathrm{~B} 4-\mathrm{CD} 3 \zeta\end{array}$ \\
\hline 3 & $\begin{array}{l}\mathrm{Li} \quad \text { (Cancer } \text { Gene } \\
\text { Therapy 2010) }\end{array}$ & PB-NK & & & $\mathrm{x}$ & & & CD19 & & $\mathrm{x}$ & & CD137-CD3ろ \\
\hline 4 & $\begin{array}{l}\text { Shimasaki } \\
\text { (Cytotherapy 2012) }\end{array}$ & PB-NK & & & $x$ & & & CD19 & & $\mathrm{x}$ & & CD137-CD3ろ \\
\hline 5 & $\begin{array}{l}\text { Boissel (Leukemia } \\
\text { Lymphoma 2012) }\end{array}$ & $\begin{array}{l}\text { PB-NK I } \\
\text { CB-NK }\end{array}$ & & $\mathrm{x}$ & $x$ & & & $\begin{array}{l}\text { GFP, CD19, } \\
\text { CD20 }\end{array}$ & $x$ & & & $\mathrm{CD} 3 \zeta$ \\
\hline 6 & $\begin{array}{l}\text { De Oliveira (Hum Gene } \\
\text { Ther 2013) }\end{array}$ & HSC & & 2 & & & & CD19 & $x$ & $x$ & & $\begin{array}{l}\mathrm{CD} 3 \zeta \text { / CD28- } \\
\mathrm{CD} 3 \zeta\end{array}$ \\
\hline 7 & $\begin{array}{l}\text { Chu (Cancer Immunol } \\
\text { Res 2015) }\end{array}$ & PB-NK & & & $x$ & & & CD20 & & $x$ & & CD137-CD3ろ \\
\hline 8 & $\begin{array}{l}\text { Suerth (J Mol Med } \\
\text { 2016) }\end{array}$ & $\begin{array}{l}\text { PB-NK + } \\
\text { NKL }\end{array}$ & $\mathrm{a} / \mathrm{g}$ & 3 & & & & CD19 & & & $x$ & $\begin{array}{l}\text { CD28-CD137- } \\
\text { CD3ろ }\end{array}$ \\
\hline 9 & $\begin{array}{l}\text { Oelsner (Int J Cancer } \\
\text { 2016) }\end{array}$ & CIK & & 2 & & & & CD19 & $x$ & $\mathrm{x}$ & & 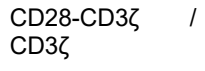 \\
\hline 10 & $\begin{array}{ll}\text { Chu } & \text { (Oncolmmunol } \\
2017) & \\
\end{array}$ & PB-NK & & & $x$ & & & CD20 & & $x$ & & CD137-CD3ろ \\
\hline 11 & $\begin{array}{l}\text { Kailayangiri } \\
\text { (Oncolmmunol 2017) }\end{array}$ & PB-NK & $x$ & & & & & GD2 & & $x$ & $x$ & 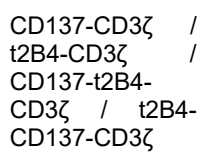 \\
\hline 12 & $\begin{array}{l}\text { Kloess (Hum Gene } \\
\text { Ther 2017) }\end{array}$ & PB-NK & $a$ & & & & & CD123 & & & $\mathrm{x}$ & $\begin{array}{l}\text { CD28-CD137- } \\
\text { CD3ろ }\end{array}$ \\
\hline 13 & Liu (Leukemia 2018) & CB-NK & $x$ & & & & & CD19 & & $x$ & & CD28-CD3ろ \\
\hline 14 & $\begin{array}{l}\text { Oei (Cancer Immunol } \\
\text { Res 2018) }\end{array}$ & PB-NK & & & $\mathrm{x}$ & & & CD19 & & $x$ & $x$ & 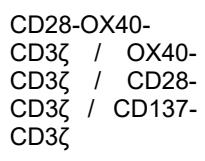 \\
\hline 15 & Yu (Mol Ther 2018) & PB-NK & & 2 & & & & GPC3 & & $\mathrm{x}$ & & CD28-CD3ろ \\
\hline
\end{tabular}




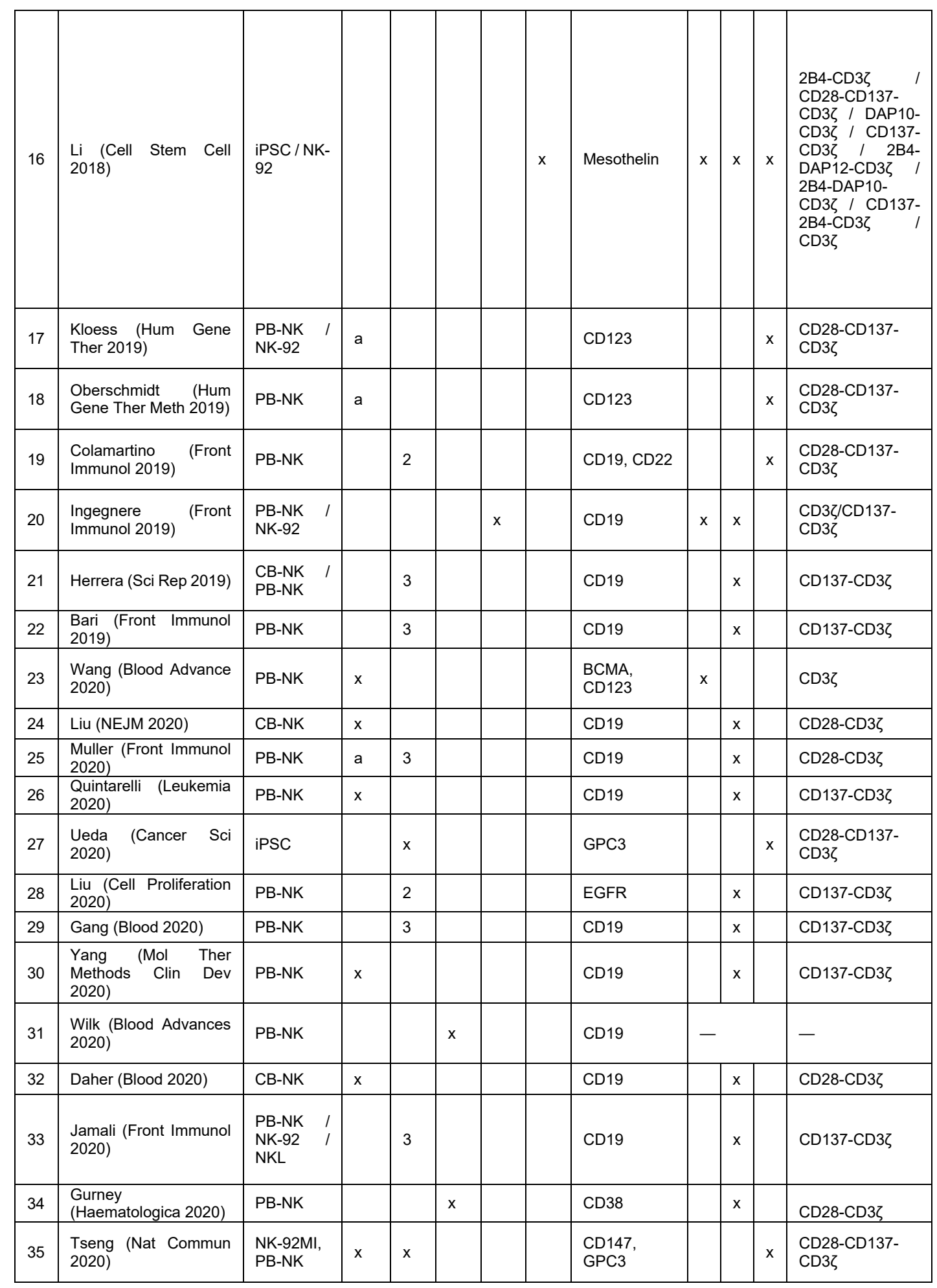


138 | Chapter 4

Numbers in the "Vehicle" column refer to the lentivirus generation. For retroviruses: a = alpha, $\mathrm{g}$ = gamma. - denotes information not provided in the study. Other abbreviations: gen. = CAR generation. Lenti $=$ lentivirus. Retro $=$ retrovirus. Transp $=$ transposon. PB-NK = peripheral blood-derived NK cells. CB-NK = cord blood-derived NK cells. CIK = Cytokineinduced killer cells.

There is an interactive and extended version of this table available online, also providing regular updates: http://www.carnkreview.com 


\section{Table 3. Next-generation CAR-NK cells.}

\begin{tabular}{|c|c|c|c|}
\hline Enhancement strategy & Target & Aim & References \\
\hline \multirow[t]{4}{*}{ Cytokine co-expression } & $\mathrm{IL}-12$ & \multirow{4}{*}{$\begin{array}{l}\text { Improve persistence } \\
\text { Improve anti-tumor } \\
\text { activity }\end{array}$} & Koneru, 2015 [212] \\
\hline & & & Pegram, 2012 [213] \\
\hline & $\mathrm{IL}-15$ & & $\begin{array}{l}\text { Liu, } 2020[30] \\
\text { Krenciute, 2017 [214] }\end{array}$ \\
\hline & $\mathrm{IL}-18$ & & $\begin{array}{l}\text { Avanzi, } 2018 \text { [215] } \\
\mathrm{Hu}, 2017 \text { [216] }\end{array}$ \\
\hline $\begin{array}{l}\text { Cytokine / JAK/STAT co- } \\
\text { expression }\end{array}$ & IL-2R $\beta$ + STAT3/5 & $\begin{array}{l}\text { Improve persistence } \\
\text { Improve anti-tumor } \\
\text { activity }\end{array}$ & Kagoya, $2018[217]$ \\
\hline $\begin{array}{l}\text { Cytokine receptor co- } \\
\text { expression }\end{array}$ & IL-7Ra & $\begin{array}{l}\text { Improve persistence } \\
\text { Improve anti-tumor } \\
\text { activity }\end{array}$ & Shum, 2017 [218] \\
\hline $\begin{array}{l}\text { Chemokine } \\
\text { expression }\end{array}$ & $\begin{array}{lr}\text { CCR2, } & \text { CCR2b, } \\
\text { CCR4, } & \text { CCR7, } \\
\text { CXCR2, } & \text { CXCR4 }\end{array}$ & $\begin{array}{l}\text { Promote trafficking into } \\
\text { tumor microenvironment }\end{array}$ & $\begin{array}{l}\text { Brown, } 2007[219] \\
\text { Craddock, } 2010[220] \\
\text { Moon, } 2011[221] \\
\text { Rapp, } 2016[222] \\
\text { Di Stasi, } 2009[223] \\
\text { Carlsten, } 2016[224] \\
\text { Kershaw, } 2002[225] \\
\text { Hillerdal, } 2015 \text { [226] } \\
\end{array}$ \\
\hline $\begin{array}{l}\text { Dual CAR (two antigens } \\
\text { required for activation) }\end{array}$ & $\mathrm{BCMA}+\mathrm{CS} 1$ & $\begin{array}{l}\text { Enhanced safety and } \\
\text { efficacy }\end{array}$ & Chen, 2018 [227] \\
\hline $\begin{array}{l}\text { Split CAR (separation of } \\
\text { co-stimulatory domains) }\end{array}$ & PSMA + PSCA & $\begin{array}{l}\text { Achieve tumor-specificity } \\
\text { in the absence of a truly } \\
\text { tumor-restricted antigen }\end{array}$ & Kloss, 2013 [228] \\
\hline \multirow{2}{*}{$\begin{array}{l}\text { Multi-antigen targeting } \\
\text { (bi-specific CAR) }\end{array}$} & HER2 + IL13Ra2 & \multirow{2}{*}{$\begin{array}{l}\text { Improve anti-tumor } \\
\text { activity } \\
\text { Prevent antigen escape }\end{array}$} & Hegde, 2016 [229] \\
\hline & $\begin{array}{l}\text { HER2 + IL13Ra2 + } \\
\text { EphA2 }\end{array}$ & & Bielamowicz, 2018 [230] \\
\hline \multirow[t]{3}{*}{ Universal CAR } & CD16 (Fc receptor) & \multirow{3}{*}{$\begin{array}{l}\text { Precise control of CAR } \\
\text { reactivity based on } \mathrm{Ab} \\
\text { half-life } \\
\text { Re-use of approved } \\
\text { antibodies, requiring only } \\
\text { one CAR construct }\end{array}$} & Caratelli, 2017 [231] \\
\hline & Antibody tag & & $\begin{array}{l}\text { Tamada, } 2012 \text { [232] } \\
\text { Feldmann, } 2017 \text { [233] }\end{array}$ \\
\hline & FITC & & Tamada, 2012 [232] \\
\hline Inhibitory CAR & $\begin{array}{lr}\text { Healthy } & \text { tissue } \\
\text { antigen, e.g. CD19 } \\
\text { (with inhibitory } \\
\text { domain, e.g. PD-1, } \\
\text { CTLA-4) }\end{array}$ & $\begin{array}{l}\text { Improve specificity, better } \\
\text { discrimination healthy } \\
\text { and tumor tissue }\end{array}$ & Fedorov, 2013 [234] \\
\hline \multirow[t]{2}{*}{$\begin{array}{l}\text { Controllable } \\
\text { expression systems }\end{array}$} & Syn/Notch & $\begin{array}{l}\text { Controlled } \\
\text { expression }\end{array}$ & Morsut, 2016 [235] \\
\hline & $\begin{array}{l}\text { Inducible } \\
\text { stimulation }\end{array}$ & Inducible CAR activation & Mata, 2017 [236] \\
\hline $\begin{array}{l}\text { Knockout of checkpoint } \\
\text { inhibitor }\end{array}$ & PD-1 & $\begin{array}{l}\text { Improve persistence } \\
\text { Improve anti-tumor } \\
\text { activity }\end{array}$ & $\begin{array}{l}\text { Rupp, } 2017 \text { [237] } \\
\text { Ren, } 2017 \text { [238] } \\
\text { Cherkassy, 2016 [203] } \\
\text { Daher, } 2021[70] \\
\end{array}$ \\
\hline
\end{tabular}

\section{Examples of enhancements to CAR constructs, mostly derived from the CAR-T field}




\section{Table 4. Comparison of virus- and non-virus mediated CAR delivery to}

\section{NK cells.}

\begin{tabular}{|c|c|c|c|}
\hline Method & Advantages & Disadvantages & GMP compliance \\
\hline Lentivirus & $\begin{array}{l}\text { High efficiency } \\
\text { Favorable safety profile } \\
\text { Transduction of resting NK }\end{array}$ & $\begin{array}{l}\text { Potential genotoxicity owing to } \\
\text { LTR sequences } \\
\text { Difficult to obtain high titer LV }\end{array}$ & $\begin{array}{l}3^{\text {rd }} \text { generation is } \\
\text { considered safe } \\
\text { enough for clinical use }\end{array}$ \\
\hline Retrovirus & $\begin{array}{l}\text { Long history in clinical trials } \\
\text { Relatively easy to produce }\end{array}$ & $\begin{array}{l}\text { Risk of insertional oncogenesis } \\
\text { Transgene capacity is lower } \\
\text { than LV } \\
\text { Requires actively dividing cells }\end{array}$ & $\begin{array}{l}\text { Compatible; } \\
\text { successfully used to } \\
\text { generate CAR-NK for } \\
\text { clinical trials }\end{array}$ \\
\hline $\begin{array}{l}\text { mRNA } \\
\text { electroporation }\end{array}$ & $\begin{array}{l}\text { Cheaper than viral production } \\
\text { No integration risks } \\
\text { High transduction rates }\end{array}$ & Transient expression & Compatible \\
\hline Transposon & $\begin{array}{l}\text { Transgene capacity higher than for } \\
\text { viruses } \\
\text { Cheap, easy to produce }\end{array}$ & Risk of insertion into genome & Compatible \\
\hline CRISPR/Cas9 & $\begin{array}{l}\text { Precise gene modification } \\
\text { Ability to use endogenous promoter } \\
\text { of choice }\end{array}$ & $\begin{array}{l}\text { Complicated to design the HDR } \\
\text { template } \\
\text { Efficiency is hard to control }\end{array}$ & $\begin{array}{l}\text { Not yet applied in } \\
\text { CAR-NK clinical trials }\end{array}$ \\
\hline
\end{tabular}

Abbreviations: $L V=$ lentivirus; HDR = homology-directed repair 


\section{Table 5. NK cell sources.}

\begin{tabular}{llll}
\hline NK cell source & Advantages & Disadvantages \\
\hline $\begin{array}{l}\text { Peripheral } \\
\text { cell }\end{array}$ & Blood NK & $\begin{array}{l}\text { Commonly used source; } \\
\text { Mature NK cells; } \\
\text { Easily expanded ex vivo }\end{array}$ & $\begin{array}{l}\text { Low Transduction efficiency; } \\
\text { Expansion induces telomere shortening } \\
\text { and reduced cytotoxicity; } \\
\text { Heterogeneity }\end{array}$ \\
\hline Cord blood derived NK & $\begin{array}{l}\text { High proliferative capacity; } \\
\text { Safety and persistence demonstrated } \\
\text { in CAR-NK trials }\end{array}$ & $\begin{array}{l}\text { Low expression of natural cytotoxicity } \\
\text { receptors; } \\
\text { Heterogeneity }\end{array}$ \\
\hline $\begin{array}{l}\text { Stem cell-derived NK } \\
\text { cells }\end{array}$ & $\begin{array}{l}\text { Off-the-shelf product; } \\
\text { Homogeneity; } \\
\text { Less cytokine release but still high } \\
\text { killing capacity. }\end{array}$ & $\begin{array}{l}\text { Potentially immunogenic; } \\
\text { Potential of malignant transformation; }\end{array}$ \\
\hline $\begin{array}{l}\text { NK-92 and other NK } \\
\text { cell lines }\end{array}$ & $\begin{array}{l}\text { Off-the-shelf product; } \\
\text { Stable expression of CAR on the } \\
\text { surface; } \\
\text { Irradiated CAR-NK-92 cell already used } \\
\text { in clinical trials; }\end{array}$ & $\begin{array}{l}\text { Tumor-derived cells, requires irradiation. } \\
\text { Irradiated CAR-NK-92 cells are not } \\
\text { sustained in vivo; } \\
\text { Not capable of ADCC (without additional } \\
\text { modifications) }\end{array}$ \\
\hline
\end{tabular}


142 | Chapter 4

Table 6. Approaches for NK cell expansion.

\begin{tabular}{|c|c|c|}
\hline Approach & Advantages & Disadvantages \\
\hline Cytokine mixture & $\begin{array}{l}\text { Feeder free } \\
\text { Easy }\end{array}$ & $\begin{array}{l}\text { Relatively long procedure; } \\
\text { May induce NK cell exhaustion }\end{array}$ \\
\hline $\begin{array}{l}\text { K562 or other feeder cell-based } \\
\text { expansion }\end{array}$ & $\begin{array}{l}\text { Rapid expansion; } \\
\text { Elongation of telomeres (only with } \\
\text { IL21) }\end{array}$ & $\begin{array}{l}\text { Risks from the feeder cells } \\
\text { (contamination, tumorigenesis) }\end{array}$ \\
\hline Autologous PBMC feeder cells & $\begin{array}{l}\text { Safe; } \\
\text { Well-known }\end{array}$ & $\begin{array}{l}\text { Need for autologous cells; } \\
\text { Expansion limited compared to } \\
\text { other techniques }\end{array}$ \\
\hline Super agonist-based expansion & $\begin{array}{l}\text { Feeder free; } \\
\text { In vivo expansion }\end{array}$ & Technology is complicated \\
\hline
\end{tabular}




\section{Table 7. Registered CAR-NK clinical trials.}

\begin{tabular}{|c|c|c|c|c|c|c|c|c|}
\hline Origin & $\begin{array}{l}\text { NCT } \\
\text { Number }\end{array}$ & Target & Extra feature & $\begin{array}{l}\text { Source } \\
\text { of NK } \\
\text { cells }\end{array}$ & $\begin{array}{l}\text { Indicatio } \\
\mathrm{n}\end{array}$ & $\begin{array}{l}\text { Trial } \\
\text { Phase } \\
\text { (Ph) }\end{array}$ & $\begin{array}{l}\text { Trial } \\
\text { Status }\end{array}$ & $\begin{array}{l}\text { Sponsor } \\
\text { and country }\end{array}$ \\
\hline \multirow[t]{16}{*}{$\begin{array}{l}\text { Industr } \\
y\end{array}$} & $\begin{array}{l}\text { GDCT0392 } \\
921\end{array}$ & EGFR & Daratumumab & $\begin{array}{l}\text { FcRY- } \\
\text { deficient } \\
\text { NK (PB- } \\
\text { derived) }\end{array}$ & $\begin{array}{l}\text { Glioblasto } \\
\text { ma }\end{array}$ & $\mathrm{Ph} 0$ & Planned & $\begin{array}{l}\text { Indapta } \\
\text { Therapeutics } \\
\text {, USA }\end{array}$ \\
\hline & $\begin{array}{l}\text { GDCT0392 } \\
823\end{array}$ & $\begin{array}{l}\text { NKG2 } \\
\text { D }\end{array}$ & None & $\begin{array}{l}\text { Allogene } \\
\text { ic, off- } \\
\text { the- } \\
\text { shelve }\end{array}$ & $\begin{array}{l}\text { AML } \\
\text { MDS } \\
\text { liver } \\
\text { cancer }\end{array}$ & $\mathrm{Ph} 0$ & Planned & NKarta, USA \\
\hline & $\begin{array}{l}\text { Not } \\
\text { available }\end{array}$ & EGFR & None & $\begin{array}{l}\text { iPSC- } \\
\text { derived } \\
\text { NK }\end{array}$ & $\begin{array}{l}\text { Glioblasto } \\
\text { ma }\end{array}$ & $\mathrm{Ph} 0$ & Planned & $\begin{array}{l}\text { Cytova } \\
\text { Therapeutics } \\
\text {, USA }\end{array}$ \\
\hline & $\begin{array}{l}\text { NCT04050 } \\
709\end{array}$ & Her2 & PD-L1 & $\begin{array}{l}\text { NK-92 } \\
\text { cell line }\end{array}$ & $\begin{array}{l}\text { Locally } \\
\text { Advanced } \\
\text { Solid } \\
\text { Tumor }\end{array}$ & $\mathrm{Ph} \mathrm{I}$ & $\begin{array}{l}\text { Ongoing, } \\
\text { recruitin } \\
\mathrm{g}\end{array}$ & $\begin{array}{l}\text { NantKwest } \\
\text { Inc, USA }\end{array}$ \\
\hline & $\begin{array}{l}\text { NCT04052 } \\
061\end{array}$ & CD19 & None & $\begin{array}{l}\text { NK-92 } \\
\text { cell line }\end{array}$ & $\begin{array}{l}\text { B Cell } \\
\text { Lymphom } \\
\text { a }\end{array}$ & $\mathrm{Ph} \mathrm{I}$ & Ongoing & $\begin{array}{l}\text { NantKwest } \\
\text { Inc, USA }\end{array}$ \\
\hline & $\begin{array}{l}\text { NCT03692 } \\
663\end{array}$ & PSMA & $\begin{array}{l}\text { Cyclophospha } \\
\text { mide }\end{array}$ & $\begin{array}{l}\text { NK-92 } \\
\text { cell line }\end{array}$ & $\begin{array}{l}\text { Prostate } \\
\text { Cancer }\end{array}$ & $\mathrm{Ph} \mathrm{I}$ & Ongoing & $\begin{array}{l}\text { NantKwest } \\
\text { Inc, USA }\end{array}$ \\
\hline & $\begin{array}{l}\text { Not } \\
\text { available }\end{array}$ & $\begin{array}{l}\text { CD19/ } \\
\text { CD22 }\end{array}$ & unknown & PB-NK & B-ALL & $\mathrm{Ph} \mathrm{I}$ & Planned & $\begin{array}{l}\text { Avalon } \\
\text { GloboCare } \\
\text { Corp, China }\end{array}$ \\
\hline & $\begin{array}{l}\text { Not } \\
\text { available }\end{array}$ & $\begin{array}{l}\text { CD19/ } \\
\text { CD22 }\end{array}$ & unknown & PB-NK & $\begin{array}{l}\text { Non- } \\
\text { Hodgkin } \\
\text { Lymphom }\end{array}$ & $\mathrm{Ph} \mathrm{I}$ & Planned & $\begin{array}{l}\text { Avalon } \\
\text { GloboCare } \\
\text { Corp, China }\end{array}$ \\
\hline & $\begin{array}{l}\text { Not } \\
\text { available }\end{array}$ & $\begin{array}{l}\text { EpCA } \\
M\end{array}$ & unknown & $\begin{array}{l}\text { NK101 } \\
\text { cell line }\end{array}$ & $\begin{array}{l}\text { Ovarian } \\
\text { Cancer }\end{array}$ & $\mathrm{Ph} \mathrm{I}$ & Planned & $\begin{array}{l}\text { SL Bigen } \\
\text { Inc, South } \\
\text { Korea }\end{array}$ \\
\hline & $\begin{array}{l}\text { Not } \\
\text { available }\end{array}$ & IL13Ra & unknown & $\begin{array}{l}\text { NK101 } \\
\text { cell line }\end{array}$ & $\begin{array}{l}\text { Glioblasto } \\
\text { ma }\end{array}$ & $\mathrm{Ph} \mathrm{I}$ & Planned & $\begin{array}{l}\mathrm{SL} \quad \text { Bigen } \\
\text { Inc, South } \\
\text { Korea }\end{array}$ \\
\hline & $\begin{array}{l}\text { Not } \\
\text { available }\end{array}$ & FLT3 & unknown & $\begin{array}{l}\text { NK101 } \\
\text { cell line }\end{array}$ & $\mathrm{AM}$ & $\mathrm{Ph} \mathrm{I}$ & Planned & $\begin{array}{ll}\mathrm{SL} & \text { Bigen } \\
\text { Inc, } & \text { South } \\
\text { Korea }\end{array}$ \\
\hline & $\begin{array}{l}\text { Not } \\
\text { available }\end{array}$ & $\begin{array}{l}\text { BCMA } \\
\text { (GoCA } \\
\text { R-NK) }\end{array}$ & IL-15 & $\begin{array}{l}\text { Primary } \\
\text { NK cells }\end{array}$ & $\begin{array}{l}\text { Multiple } \\
\text { Myeloma }\end{array}$ & $\begin{array}{l}\text { Preclini } \\
\text { cal }\end{array}$ & Planned & $\begin{array}{l}\text { Bellicum } \\
\text { Phamaceutic } \\
\text { als }\end{array}$ \\
\hline & $\begin{array}{l}\text { NCT02944 } \\
162\end{array}$ & CD33 & None & $\begin{array}{l}\text { NK-92 } \\
\text { cell line }\end{array}$ & AML & $\mathrm{Ph} \mathrm{I}$ & $\begin{array}{l}\text { Ongoing, } \\
\text { recruitin } \\
\mathrm{g}\end{array}$ & $\begin{array}{l}\text { PersonGen } \\
\text { Biomedicine } \\
\text { Suzhou Co } \\
\text { Ltd, China }\end{array}$ \\
\hline & $\begin{array}{l}\text { NCT03941 } \\
457\end{array}$ & $\begin{array}{l}\text { ROBO } \\
1 \\
(\mathrm{BiCA} \\
\mathrm{R})\end{array}$ & None & $\begin{array}{l}\text { NK-92 } \\
\text { cell line }\end{array}$ & $\begin{array}{l}\text { Metastatic } \\
\text { Pancreati } \\
\text { c Cancer }\end{array}$ & $\mathrm{Ph} \mathrm{I} / / \mathrm{I}$ & $\begin{array}{l}\text { Ongoing, } \\
\text { recruitin } \\
\mathrm{g}\end{array}$ & $\begin{array}{l}\text { Asclepius } \\
\text { Technology } \\
\text { Company } \\
\text { Group } \\
\text { Suzhou Co } \\
\text { Ltd, China }\end{array}$ \\
\hline & $\begin{array}{l}\text { NCT03940 } \\
833\end{array}$ & BCMA & None & $\begin{array}{l}\text { NK-92 } \\
\text { cell line }\end{array}$ & $\begin{array}{l}\text { Multiple } \\
\text { Myeloma }\end{array}$ & $\mathrm{Ph} \mathrm{l} / \mathrm{II}$ & $\begin{array}{l}\text { Ongoing, } \\
\text { recruitin } \\
\mathrm{g}\end{array}$ & $\begin{array}{l}\text { Asclepius } \\
\text { Technology } \\
\text { Company } \\
\text { Group } \\
\text { Suzhou Co } \\
\text { Ltd, China }\end{array}$ \\
\hline & $\begin{array}{l}\text { NCT03940 } \\
820\end{array}$ & $\begin{array}{l}\text { ROBO } \\
1\end{array}$ & None & $\mathrm{NK} / \mathrm{T}$ & $\begin{array}{l}\text { Malignant } \\
\text { Neoplasm } \\
\mathrm{s}\end{array}$ & $\mathrm{Ph} \mathrm{I} / \mathrm{II}$ & $\begin{array}{l}\text { Ongoing, } \\
\text { recruitin } \\
\mathrm{g}\end{array}$ & $\begin{array}{l}\text { Asclepius } \\
\text { Technology } \\
\text { Company } \\
\text { Group }\end{array}$ \\
\hline
\end{tabular}




\section{4 | Chapter 4}

\begin{tabular}{|c|c|c|c|c|c|c|c|c|}
\hline & & & & & & & & $\begin{array}{l}\text { Suzhou Co } \\
\text { Ltd, China }\end{array}$ \\
\hline & $\begin{array}{l}\text { NCT02892 } \\
695\end{array}$ & CD19 & None & $\begin{array}{l}\text { NK-92 } \\
\text { cell line }\end{array}$ & Leukemia & $\mathrm{Ph} \mathrm{I/II}$ & Planned & $\begin{array}{l}\text { PersonGen } \\
\text { Biomedicine } \\
\text { Suzhou Co } \\
\text { Ltd, China }\end{array}$ \\
\hline & $\begin{array}{l}\text { NCT02742 } \\
727\end{array}$ & CD7 & None & $\begin{array}{l}\text { NK-92 } \\
\text { cell line }\end{array}$ & $\begin{array}{l}\text { AML, } \mathrm{T}- \\
\text { cell } \\
\text { leukemia, }\end{array}$ & $\mathrm{Ph} \mathrm{I/II}$ & $\begin{array}{l}\text { Recruitin } \\
\mathrm{g}\end{array}$ & $\begin{array}{l}\text { PersonGen } \\
\text { BioTherapeu } \\
\text { tics (Suzhou) }\end{array}$ \\
\hline & & & & & lymphoma & & & $\begin{array}{l}\text { Co., Ltd, } \\
\text { China }\end{array}$ \\
\hline & $\begin{array}{l}\text { NCT02839 } \\
954\end{array}$ & MUC1 & None & $\begin{array}{l}\text { NK-92 } \\
\text { cell line }\end{array}$ & $\begin{array}{l}\text { HCC, } \\
\text { NSCLC, } \\
\text { pancreatic }\end{array}$ & $\mathrm{Ph} \mathrm{I/II}$ & $\begin{array}{l}\text { Recruitin } \\
\mathrm{g}\end{array}$ & $\begin{array}{l}\text { PersonGen } \\
\text { BioTherapeu } \\
\text { tics (Suzhou) }\end{array}$ \\
\hline & & & & & $\begin{array}{l}\text { carcinoma } \\
\text { breast } \\
\text { cancer, } \\
\text { glioma, } \\
\text { CRC, } \\
\text { gastric } \\
\text { carcinoma }\end{array}$ & & & $\begin{array}{l}\text { Co., } \quad \text { Ltd, } \\
\text { China }\end{array}$ \\
\hline & $\begin{array}{l}\text { NCT04639 } \\
739\end{array}$ & CD19 & None & NK cell & $\begin{array}{l}\text { relapsed } \\
\text { and } \\
\text { refractory } \\
\text { B-cell } \\
\text { non- } \\
\text { Hodgkin } \\
\text { lymphoma }\end{array}$ & $\mathrm{Ph} 0$ & Planned & $\begin{array}{l}\text { Xinqiao } \\
\text { Hospital, } \\
\text { Chongqing, } \\
\text { China }\end{array}$ \\
\hline $\begin{array}{l}\text { Acade } \\
\text { mic }\end{array}$ & $\begin{array}{l}\text { NCT03056 } \\
339\end{array}$ & CD19 & $\begin{array}{l}\text { chemotherapy } \\
+ \text { BSCT }\end{array}$ & CB-NK & $\begin{array}{l}\text { B-cell } \\
\text { lymphoma } \\
\text { or } \\
\text { leukemia }\end{array}$ & $\mathrm{Ph} \mathrm{I/II}$ & Ongoing & $\begin{array}{l}\text { M.D. } \\
\text { Anderson } \\
\text { Cancer } \\
\text { Center, USA }\end{array}$ \\
\hline & $\begin{array}{l}\text { NCT03383 } \\
978\end{array}$ & HER2 & None & $\begin{array}{l}\text { NK-92 } \\
\text { cell line }\end{array}$ & $\begin{array}{l}\text { Glioblasto } \\
\text { ma }\end{array}$ & $\mathrm{Ph} \mathrm{I/II}$ & Ongoing & $\begin{array}{l}\text { Johann } \\
\text { Goethe } \\
\text { University } \\
\text { Hospital, } \\
\text { Germany }\end{array}$ \\
\hline & $\begin{array}{l}\text { NCT01974 } \\
479\end{array}$ & CD19 & IL-2 sc & $\begin{array}{l}\text { PB-NK } \\
\text { on } \\
\text { K562- } \\
\text { mblL15- } \\
\text { 41BBL }\end{array}$ & ALL & $\mathrm{Ph} \mathrm{I/II}$ & $\begin{array}{l}\text { Suspend } \\
\text { ed for an } \\
\text { interim } \\
\text { review }\end{array}$ & $\begin{array}{l}\text { National } \\
\text { University } \\
\text { Health } \\
\text { System, } \\
\text { Singapore }\end{array}$ \\
\hline
\end{tabular}

PB-NK = peripheral blood-derived natural killer cells. CB-NK = cord blood-derived natural killer cells. 
Box 0. My personal road to the generation of CAR-NK cells: table of contents.

The boxes in this chapter will describe the experimental road I took in the generation of CAR-NK cells. It describes the bumpy pavement and obstacles I came across along the way. The roadmap has become more detailed during the journey, but is not finished yet. As project time is running out, I still want to share the obtained knowledge and results. They fit in nicely within the extensive review of the CAR-NK cell field and as such form now one integrated chapter of my journey written in this thesis.

The 12 boxes will describe:

1) Overview of generated CAR-NK constructs.

2) Comparison between BaEV and VSV-G lentiviral envelope alternatives.

3) AAV6 and retrovirus transduction of NK cells.

4) Electroporation on human NK cells.

5) Different CAR-NK expression detection methods to be analyzed by FACS.

6) $\quad$ QPCR detection on $\alpha$-Her2-CAR transduced NK cells.

7) $\quad \alpha-C D 3 \zeta$ Western blot detection of CAR transduced NK cells.

8) Human primary NK cells expand on in house generated K-562 membrane IL21/4-1BBL feeder cells.

9) Different promoters have an effect on $\alpha$-Her2-CAR and a-MUC1-CAR expression.

10) Cytotoxicity assay of $\alpha-H e r 2-C A R$ and $\alpha-M U C 1-C A R$ NK cells towards different tumor cell lines.

11) Intracellular staining of effector molecules in primary NK cells transduced with a-Her2-CAR and $\alpha$-MUC1-CAR with different cell lines;

12) Conclusion of the boxes on the generation of CAR-NK cells plus recommendations for the future. 


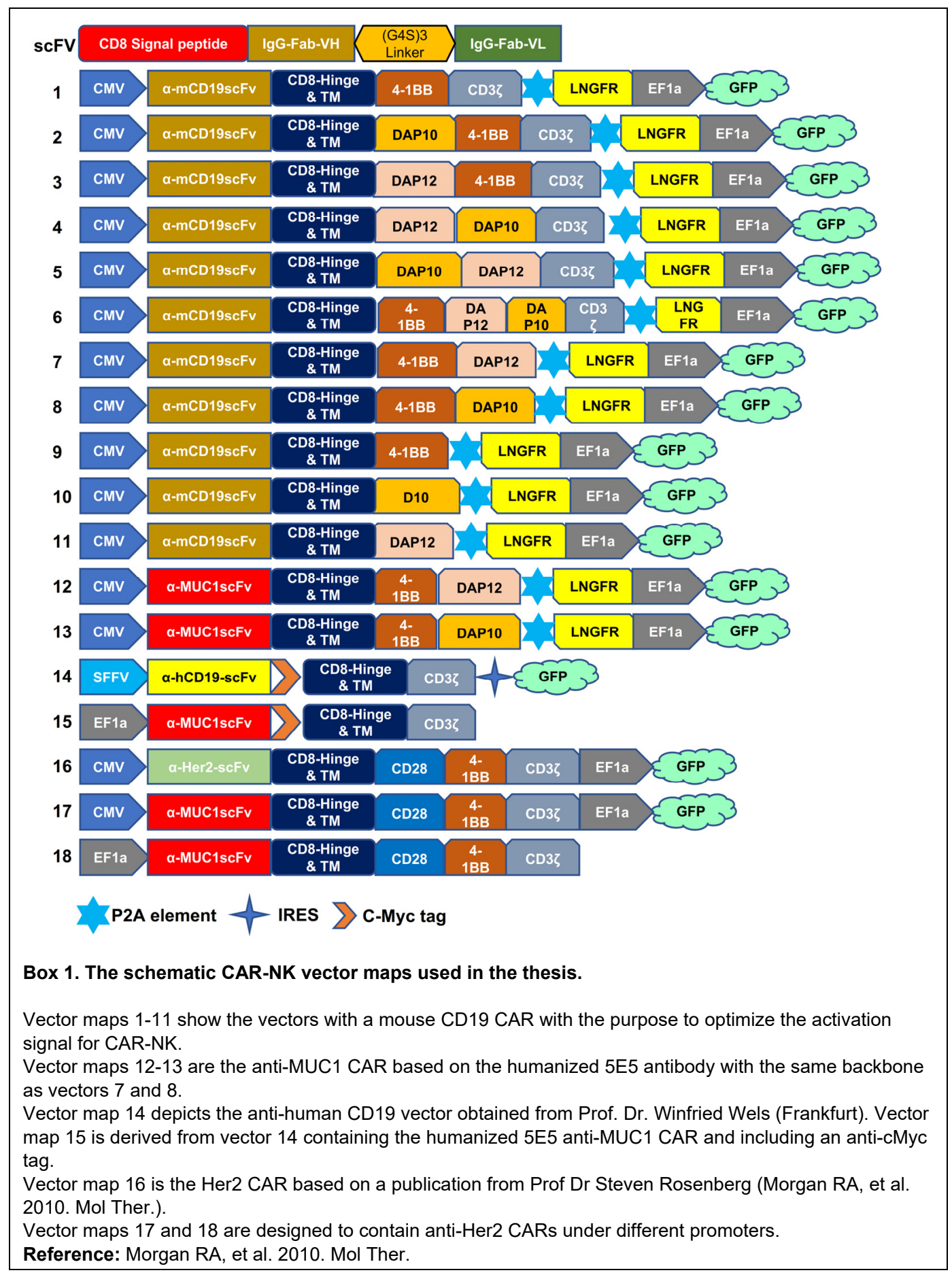




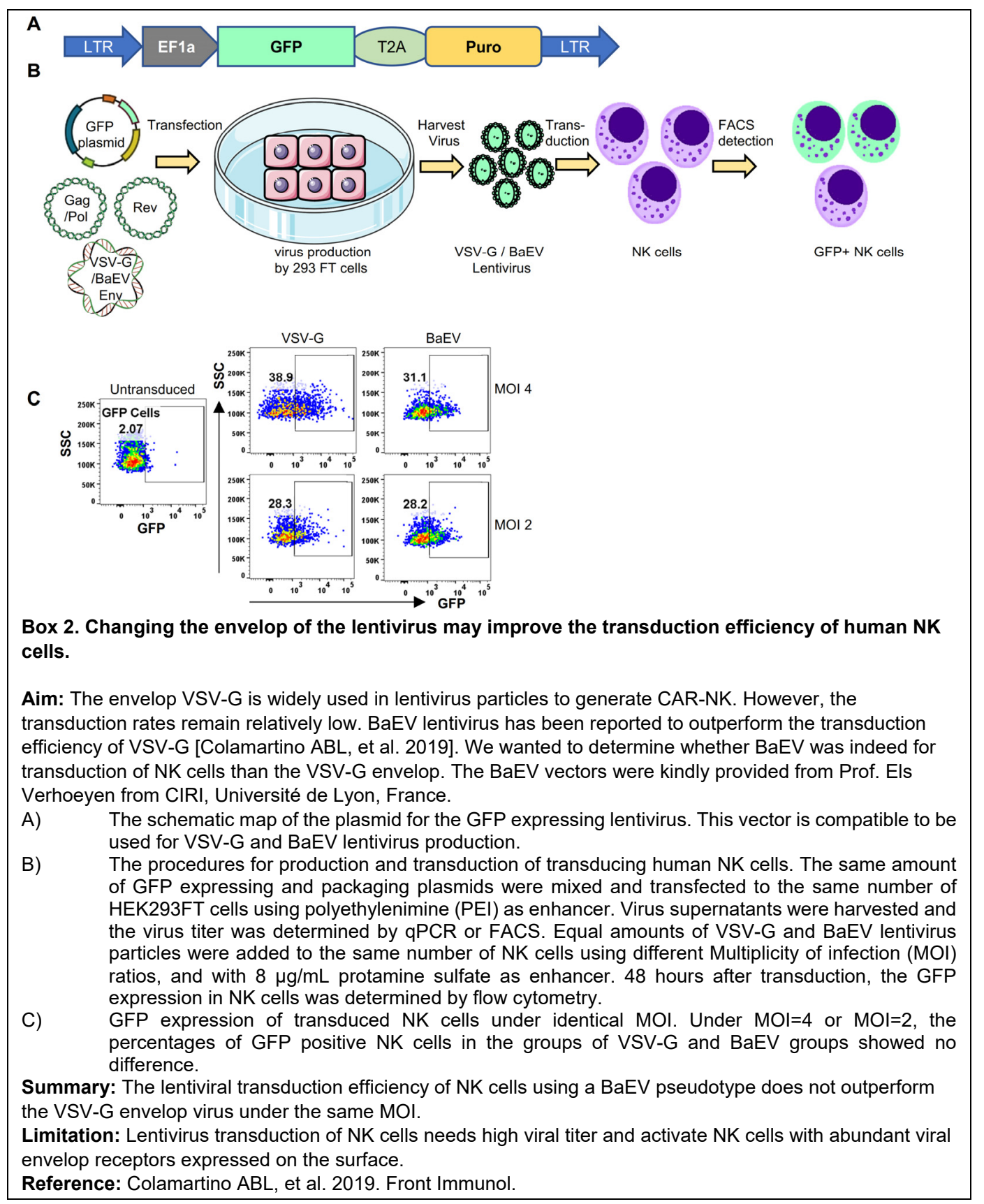




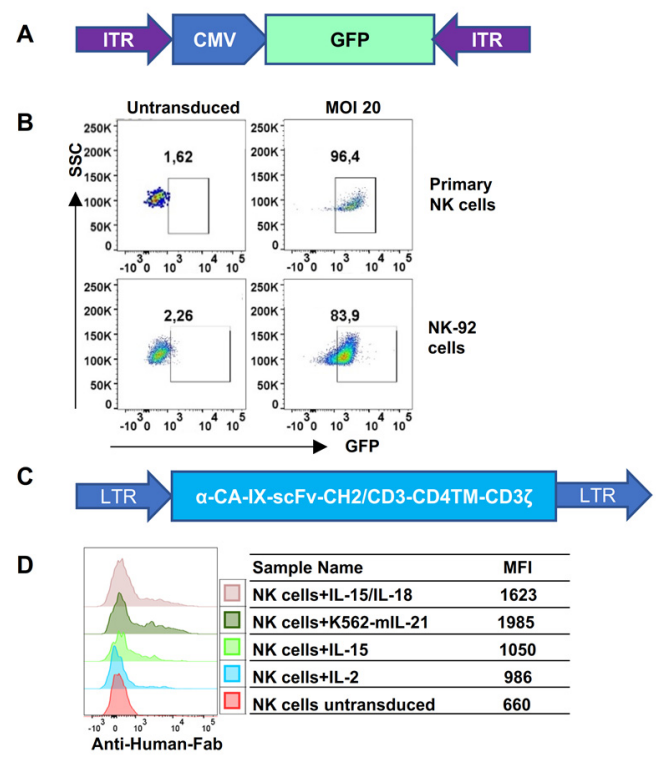

Box 3. AAV-6 and retrovirus transduction of human NK cells.

Aim: Alternative viral system may yield better results to transduce human primary NK cells. To explore whether recombinant Adeno Associated Viruses (AAV) or retroviruses are able to transduce human primary NK cells efficiently. The anti-CAIX-CAR is a positive control for the CAR-NK staining.

\section{Procedures:}

For AAV transduction: The AVV-6 serotype virus was produced in 293FT cells and added in a MOI=100 virus particles to the NK cells. After 7 Days, NK cells were harvested and after staining, the CAR expression levels were detected on a FACS machine.

For Retrovirus transduction: The anti-CAIX-CAR virus was kindly provided by Prof Reno Debets and Dr Cor Lamers, Erasmus MC+, Rotterdam. NK cells were pre-stimulated with different cytokines for 48 hours. Then retrovirus supernatant was mixed in the culture medium of NK cells in a retronectin pre-coated cell culture plate. After 48 hours, NK cells were stained with anti-human IgG Fab to checked the CAR expression.

A) A schematic vector map of the AAV GFP expressing vector.

B) The FACS plot showing GFP expression in NK cells on Day 7 after AAV-6 virus transduction. The number indicates the percentage of GFP positive NK cells. After 7 days about $90 \%$ NK cells are GFP positive in both primary NK cells and the NK-92 cell line.

C) The vector map of anti-CAIX-CD3ろ.

D) The histogram overlay of the expression of CAIX-CAR on human primary NK cells after stimulation with different cytokines. The Fab signal represents the anti-CAIX CAR expression level. NK cells stimulated with IL-2/IL-15/IL-18 or expanded on K562-mIL-21/4-1BBL enhance the retrovirus transduction efficiency.

Summary: AAV-6 virus is efficient in transducing both primary NK cells and the NK-92 cell line. Cytokine stimulation of human primary NK cells can further improve the retroviral transduction efficiency.

Limitations: AAV-6 is highly efficient in transducing human NK cells, but the AAV virus is a DNA virus that is difficult to integrate into the genome of NK cells, which means it is not able to propagate into new generations after further cell division. Moreover, the gene delivery size limitation of AAV-6 is around $4.5 \mathrm{~kb}$ between the two ITR ranges. Retrovirus gene transfer has been reported to induce leukemia due to activation of oncogenes after integration of the vector, still posing a high risk and as such not preferred to use. With these disadvantages in hand and mind, we continued to use a lentivirus for CAR-NK generation. Only the $3^{\text {rd }}$ generation of lentivirus has a good safety profile and a high transduction efficiency and therefore we will only use this type of vector to produce CAR-NK. 


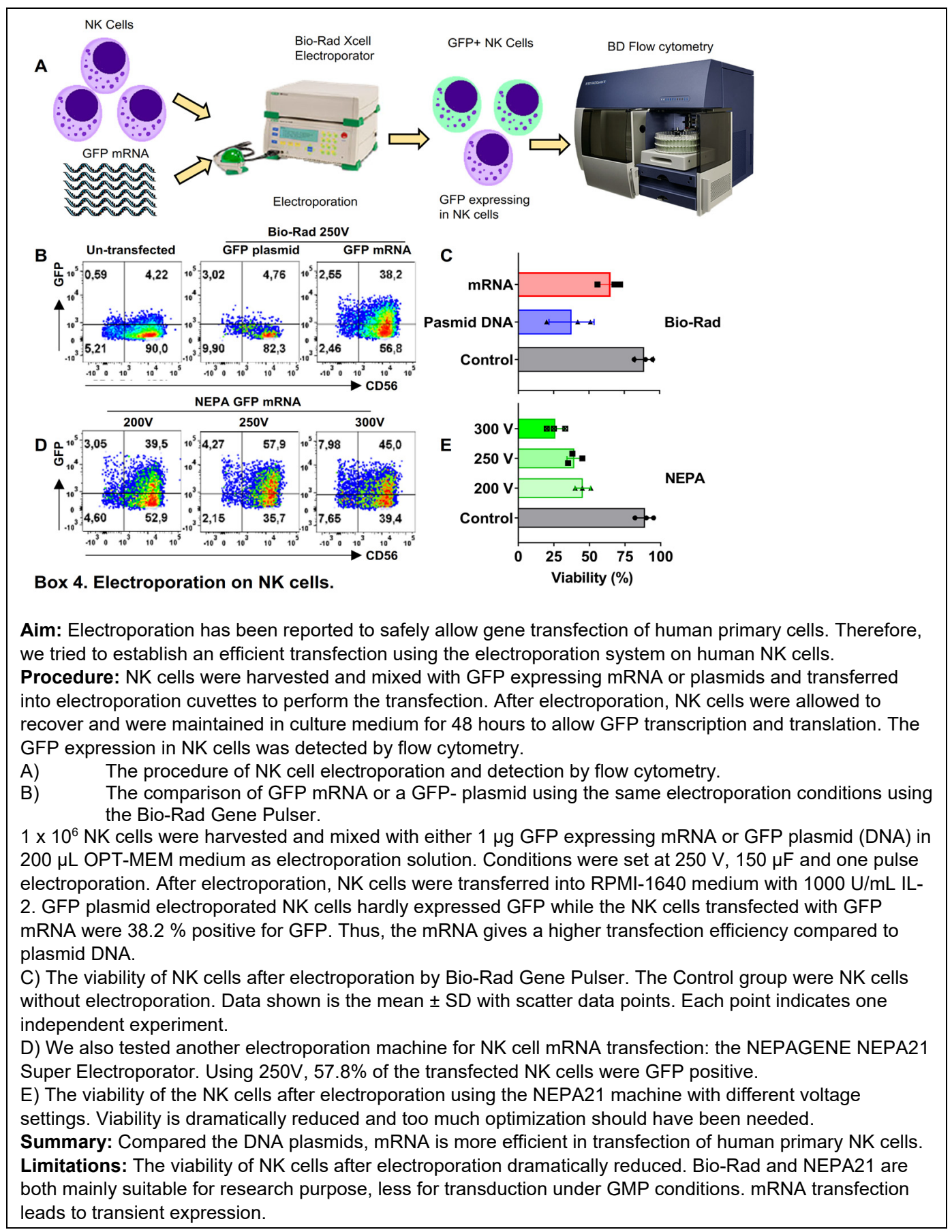




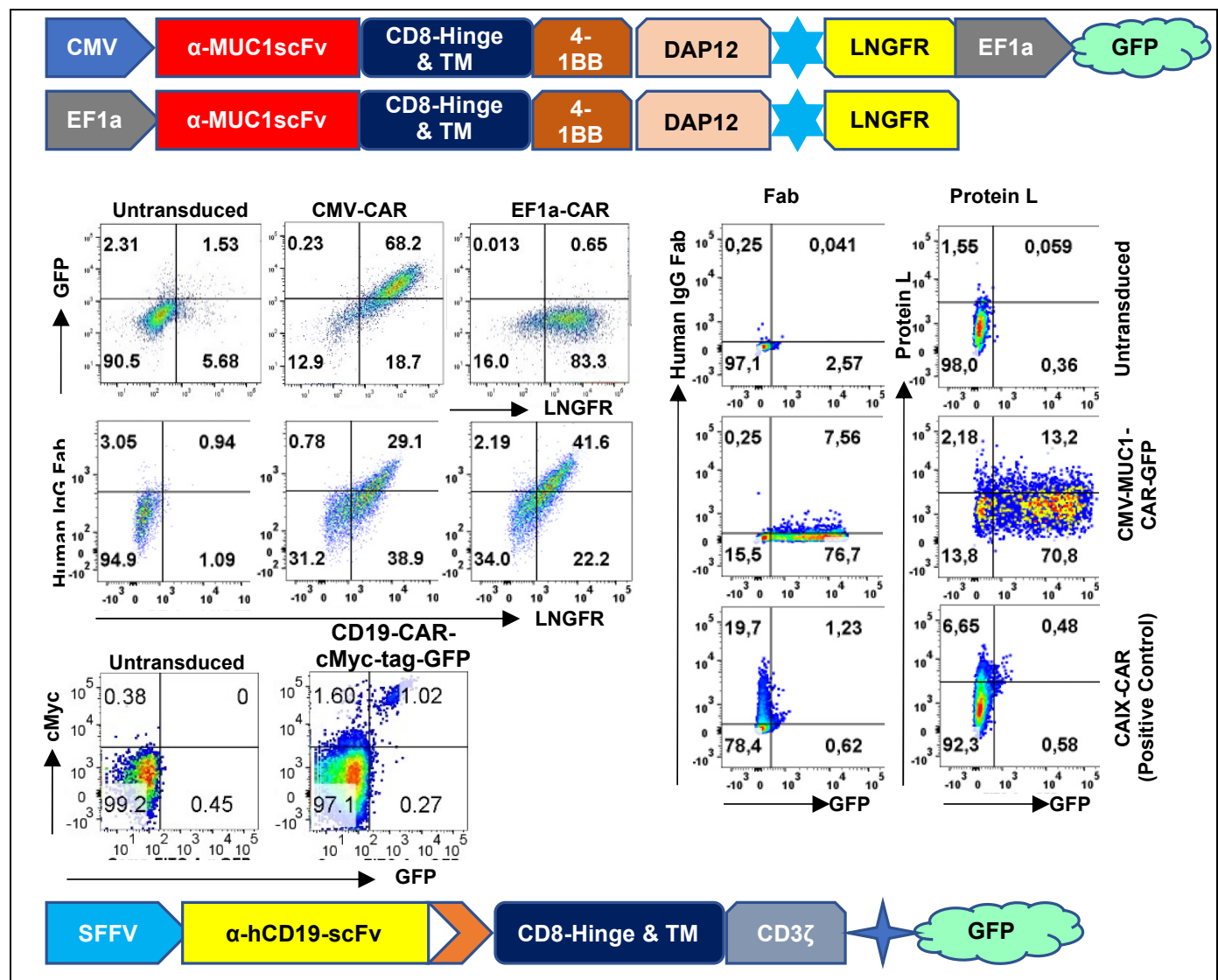

Box 5. Detection of CAR expression on NK cells using flow cytometry.

Aim: To explore the flow cytometry-based detection method for CAR expression on transduced NK cells. As CAIX-CAR retroviral supernatant was kindly provided by Prof. Reno Debets, we intended to use this to setup a positive CAR-NK detection system using FACS.

Procedures: NK cells were transduced with CAR encoding $3^{\text {rd }}$ generation lentivirus. 48 hours, or longer, after transduction, NK cells were harvested and stained with antibodies. After washing the cells with PBS, CAR expression on NK cells was analyzed by flow cytometry.

A) Schematic maps of CAR vectors.

B) Representative FACS plot of GFP and LNGFR expression after CMV-CAR or EF1a-CAR lentivirus transduction as well as the LNGFR with Fab co-expression.

C) Representative FACS plot of anti-IgG-Fab and protein-L anti-human IgG kappa chain to detect the scFv structure of the CAR. CMV-CAR-GFP and positive control CAIX-CAR were transduced in NK cells and were stained after 48 hours with anti-human-IgG Fab and protein L anti IgG kappa chain antibodies. More than $80 \%$ of the population NK cells was positive for GMP after transduction with the CMV-CAR-GFP virus, but only $7 \%$ of the NK cells were Fab positive and $15 \%$ was protein-L positive. In CAIX-CAR positive NK cells, $20 \%$ of the cells were Fab positive and $6 \%$ was protein-L positive.

D) A representative FACS plots for cMyc-tag staining of human primary NK cells $48 \mathrm{~h}$ after lentivirus transduction. A population of $1 \%$ cMyc and GFP double positive population was detected by FACS.

E) Schematic map of the anti-h-CD19-cMyc-GFP vector. This vector is kindly provided by Prof. Winfried Wels from Georg-Speyer-Haus, Institute for Tumor Biology and Experimental Therapy, Frankfurt am Main, Germany. 
Summary: The GFP and CAR expression are controlled by different promotors in the constructs while LNGFR and CAR are behind the same promoter. This difference may explain why GFP is not correlated with Fab and protein $L$ staining. Markers that are used for CAR detection should be expressed under the same promoter which is possible by including IRES/2A sites. Moreover, even with bicistronic elements, detection of the marker protein (LNGFR, or GFP if under the same promoter), does not guarantee CAR expression on the membrane. Hence, a Myc or His tag is more useful because it is integrated in the CAR protein itself. In addition, Fab staining or detection of binding of the antigen (e.g. the relevant CD19 antigen with an Fc-tail, which is then detected) is also a good way to detect a CAR protein on the surface.

Limitations: Some targets that CARs recognize may not be directly stained, thus may restrain the staining procedure. Concluding: The Fab or a tag can used as a versatile approach to detecting CAR expression on NK cells.

Reference: Zheng Z, et al. (2012). J Transl Med. 


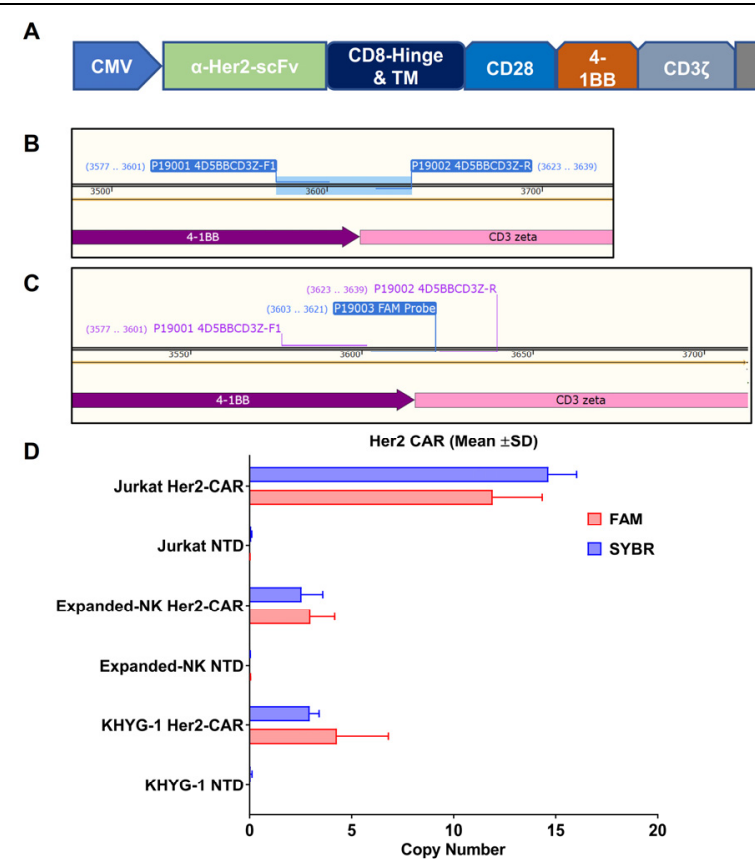

Box 6. qPCR-based CAR detection in NK cells.

Aim: To set-up methods of CAR detection by real-time qPCR with SYBR green and TaqMan probes.

Procedure: mRNA of $1 \times 10^{6}$ CAR transduced NK cells was isolated and treated with DNase I. Then, cDNA was synthesized using oligo dT primers and used as template for qPCR. Then, the SYBR and TaqMan qPCR were conducted at the same time. In parallel, the copy number of the integrated CAR construct in NK cells was determined in relation to a standard curve based on a plasmid serial dilution.

A) Schematic map of the CAR vector, containing the 4-1BB and CD3 3 gene elements adjacently as a fusion gene and is as such not present in natural NK cells.

B) The primers used in the qPCR where the forward primer locates in 4-1BB while the reverse primer is designed in the $\mathrm{CD} 3 \zeta$ fragment, creating a unique fragment only detectable when the CAR is introduced.

C) The TaqMan probe was chosen in the center of the 4-1BB and CD3 3 adjacent parts.

D) The GPCR results of SYBR and TaqMan methods of the CAR transduced NK cells.

Summary: The SYBR and TaqMan are equally sensitive to detect the CAR vector copy numbers in transduced NK cells.

Limitations: qPCR can detect the copy number of vectors in CAR transduced NK cells, but the copy number cannot predict the protein expression levels on the NK cell surface and the functionality of CAR. 


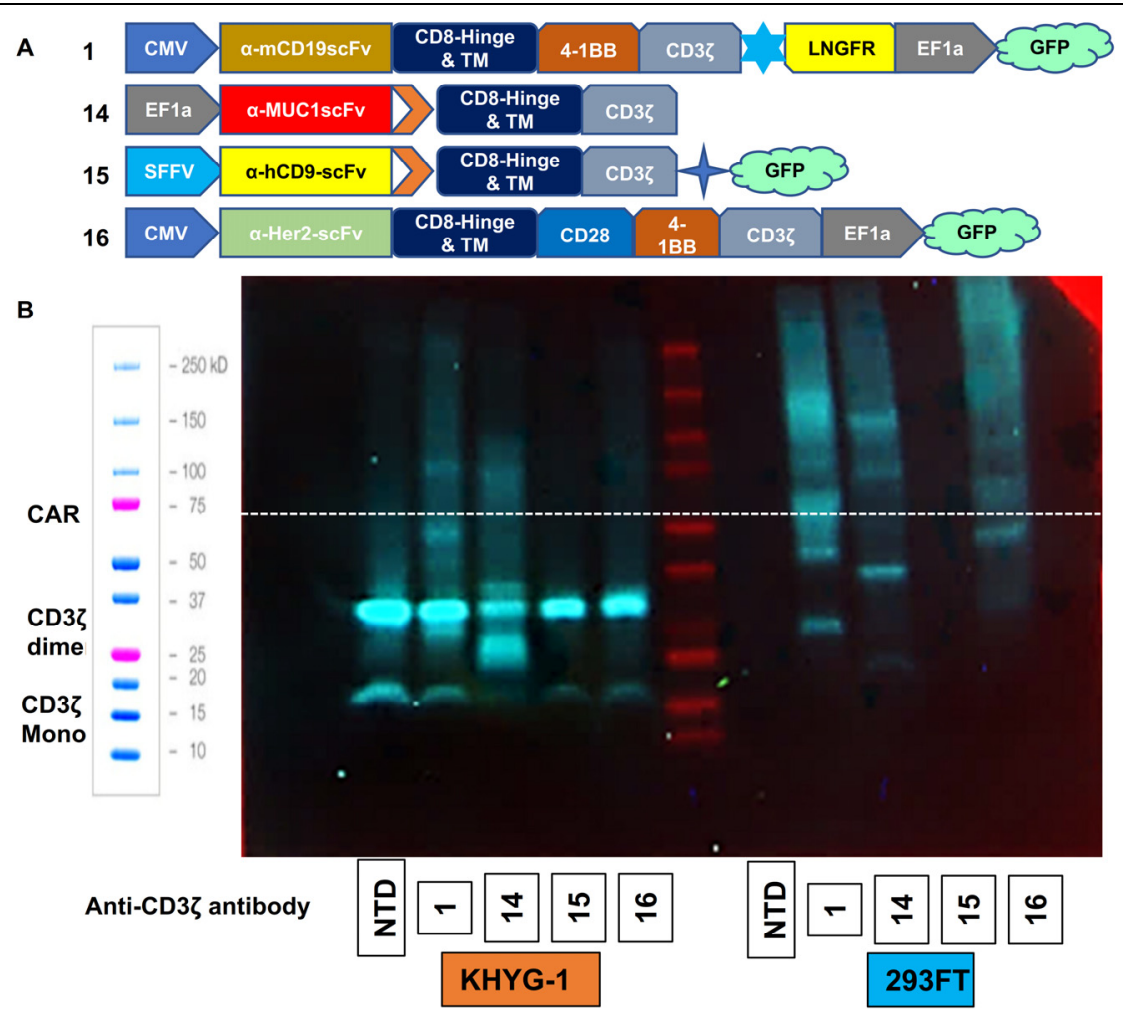

\section{Box 7. CAR expression on NK cells as detected by Western Blot analysis}

Aim: To set-up the detection method of CARs using the Western Blot technology.

Procedure: The NK cell line KHYG-1 was transduced with 4 different CAR constructs, all containing the $\mathrm{CD} 3 \zeta$ intracellular activation signal. 293FT is the lentivirus producing cell line that was transfected the CAR expressing vectors. 1 × $10^{6}$ CAR-transduced KHYG-1 and CAR-transfected 293FT cells were harvested and lysed in RIPA buffer without DTT or $\beta$-mercaptoethanol ( $\beta$-ME). After protein denaturation, the cell lysates were loaded onto a SDS-PAGE gel and subjected to electrophoresis. As a result, the protein was separated on size and transferred to a PVDF membrane. After protein transfer, the membrane was blocked with $0.5 \%$ BSA and stained with a mouse IgG anti-human-CD3 $\zeta$ primary antibody. Goat anti-mouse-lgG-HRP was used as secondary antibody for final detection. The band of the WB blot was visualized by luminescence by adding ECL Western Blotting Substrate and the light was captured by the Bio-Rad ChemiDoc MP system.

A) Vector maps of 4 CAR encoding CD3 3 constructs

B) The result of the Western blot of transduced KHYG-1 cell with 4 CAR vectors and transfected 293FT cells with the same 4 CAR vectors. The target band of the CAR is $65 \mathrm{kDa}$, which is indicated with the dashed line. In KHYG-1, there is abundant endogenous CD3 3 expression, while 293 FT lacks $\mathrm{CD} 3 \zeta$ expression (the monomer is $18 \mathrm{kDa}$, the dimer is about $36 \mathrm{kDa}$ ). After introducing the CAR, there was a smear in both KHYG-1 and 293FT cells around the expected height.

Summary: Although the CAR introduced into the cells can be detected by western blot, it generates a smeared band. Therefore, further optimization would be need for the western blot to be really useful.

Limitation: The CAR target band density is determined by the transduction or the transfection efficiency. A low transduction efficiency may induce a smeared target band. The western blot can prove that the CAR protein is present in the cells but cannot see whether the CAR is properly expressed on the NK cell surface. 

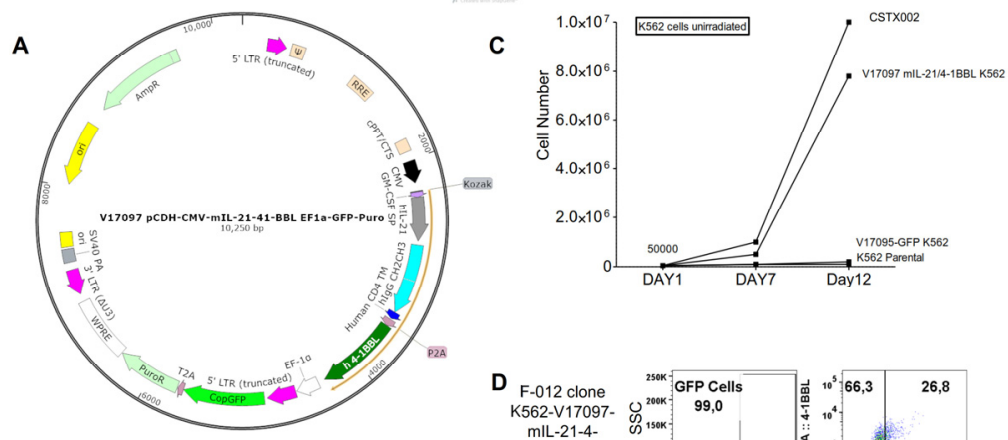

B

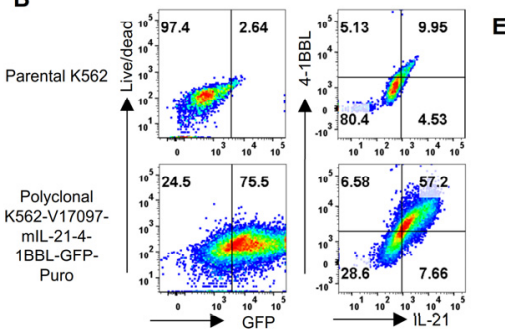

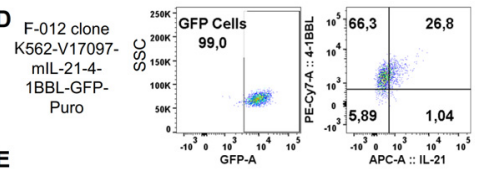

K562 mIL-21/4-1BBL clones for NK cell expansion

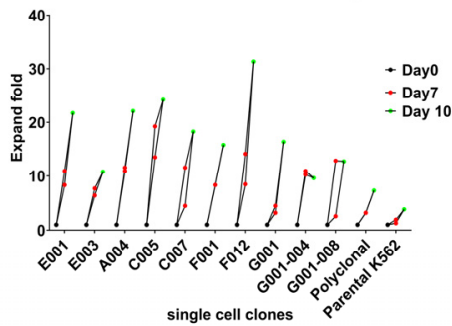

Box 8. Generation of a K562-mIL-21-4-1BBL-GFP based NK cell expansion method.

Aim: To generate an NK cells expansion method with K562-mIL-21-4-1BBL feeder cells.

Procedure: At first the feeder cell line was generated. To this end, the IL-21 (attached with a transmembrane region) and 4-1BBL genes were clone into a $3^{\text {rd }}$ generation lentivirus vector, which further contain the GFP and puromycin resistance genes. With this construct, lentiviral particles were produced in HEK293FT cells. K-562 cells were transduced with the mIL-21-4-1BBL encoding lentivirus. After puromycin selection, K562 expressed high GFP, 4-1BBL and IL-21. From the bulk, single cell clones were sorted out by flow cytometry FACS sorting. After single cells propagated, 10 clones of K562-mIL-21-4-1BBL-GFP were chosen for further verification and expansion assays. Finally, one clone was selected that showed the greatest NK cell expansion support.

A) The vector map of the $3^{\text {rd }}$ generation lentivirus expression vector encoding membrane bound IL-21 and 4-1BBL.

B) The expression levels of GFP, mIL-21 and 4-1BBL in the K-562 cells transduced with lentivirus. After puromycin selection, K-562 highly expressed GFP, mIL-21 and 4-1BBL.

C) Primary NK cell expansion using the polyclonal K562-mIL-21-4-1BBL-GFP cells in comparison to the CSTX002 obtained from Prof. Dean Lee. The parental K-562 cells and K-562-GFP only cells were used as negative control for the expansion. The newly generated K562-mIL-21-4-1BBL-GFP cells could expand human primary NK cells.

D) The representative FACS plot of GFP, IL-21 and 4-1BBL expression on K562-mIL-21-4-1BBL-GFP the single cell clone F-012.

E) The primary NK cell expansion assays by 10 K562-mIL-21-4-1BBL-GFP single cell clones in comparison to the polyclonal cells.

Summary: The K562-mIL-21-4-1BBL-GFP cells is able to expand human primary NK cells.

Limitations: The expansion fold is encouraging, although the expansion protocol needs further optimization. By now, colleagues at CiMaas have performed these experiments and the current status is that the irradiated F012 clone supports NK cells expansion so much that clinically applicable numbers $\left(3 \times 10^{10}\right)$ have repeatedly been obtained from different donors creating a protocol that is the base for a clinical trial using primary expanded NK cells. 


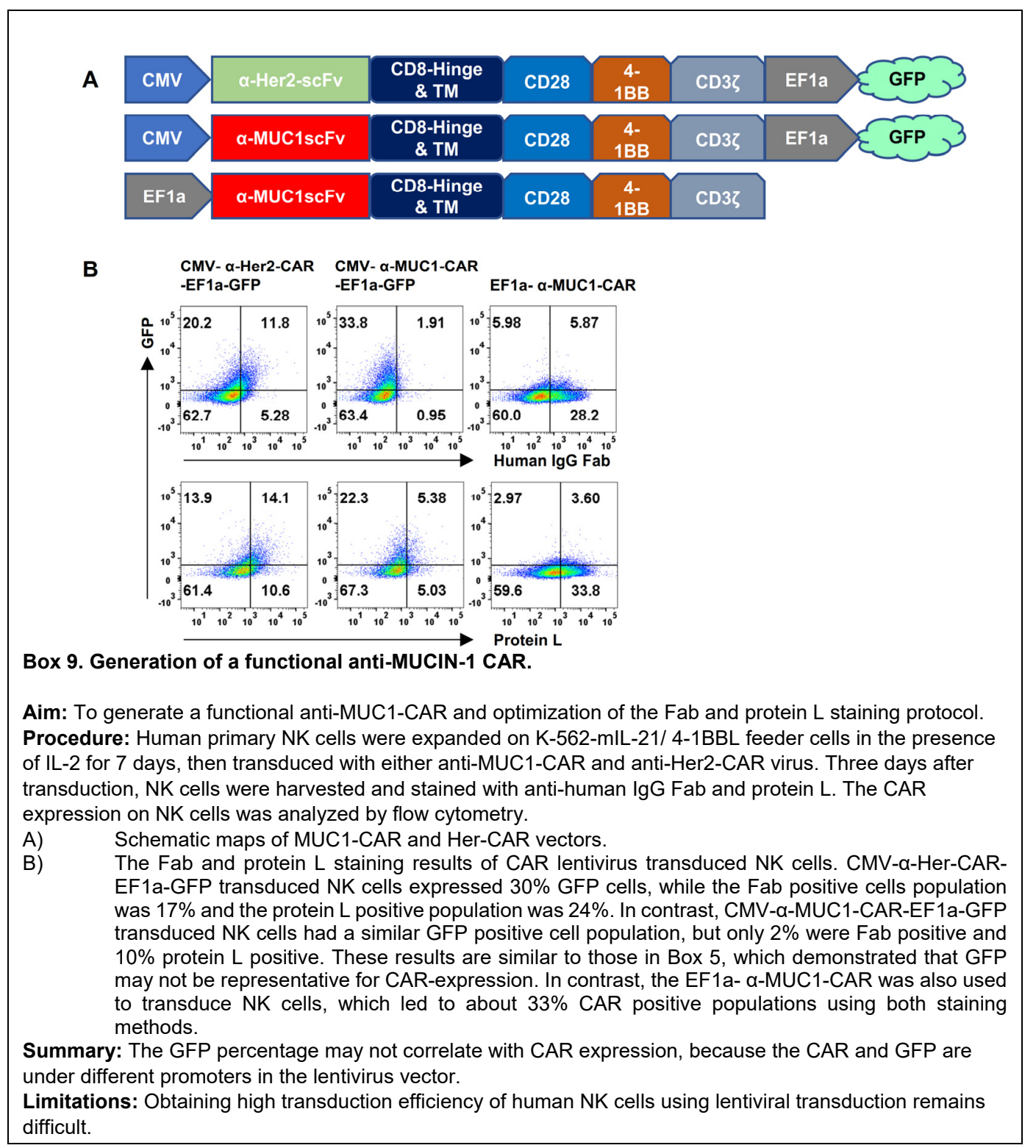


A

\begin{tabular}{|c|c|c|c|c|c|c|}
\hline CMV & a-Her2-scFv & $\begin{array}{l}\text { CD8-Hinge } \\
\text { \& TM }\end{array}$ & CD28 & $\begin{array}{c}\text { 4- } \\
1 \mathrm{BB}\end{array}$ & CD3ろ & EF1a \\
\hline CMV & $\alpha-M U C 1 s c F v$ & $\begin{array}{c}\text { CD8-Hinge } \\
\text { \& TM }\end{array}$ & CD28 & $\begin{array}{c}4- \\
1 \mathrm{BB}\end{array}$ & CD3 $\zeta$ & EF1a \\
\hline EF1a & a-MUC1scFv & $\begin{array}{c}\text { CD8-Hinge } \\
\text { \& TM }\end{array}$ & CD28 & $\begin{array}{c}4- \\
1 \mathrm{BB}\end{array}$ & CD3 $\zeta$ & \\
\hline
\end{tabular}

B

K562 (Her2-, MUC1-)

T-47D (Her2+, MUC1+)

Jurkat (Her2-, MUC1+)
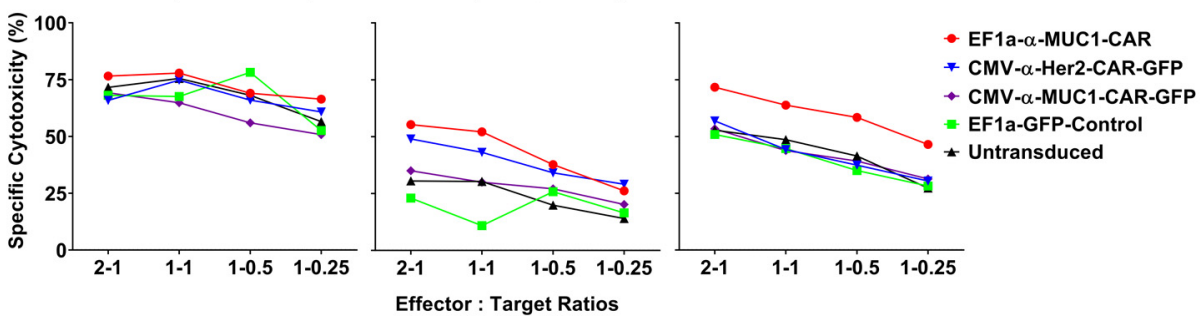

Box 10. Cytotoxicity of CAR transduced primary NK cells.

Aim: To validate the cytotoxicity potential of virus transduced NK cells expressing a CAR.

Procedure: After verification of CAR expression Fab and protein L CAR detection methods, NK cells were harvested and cytotoxicity assays were conducted to validate the functionality of CAR-NK cells. Tumor cells (K-562, T-47D and Jurkat) with or without Her2 and MUC1-Tn antigens were used as the target cells and labelled with CIM-DiL dye. Target cells in a fixed cell number of 20,000 cells per condition in a 96-well plate, NK cell were seeded in different effector : target ratios. After $4 \mathrm{~h}$ co-culturing, tumor cells were stained with the live/dead staining dye aqua-V500. Dead tumor cells were positive for the V500 signal and analyzed by flow cytometry.

A) Schematic maps of CAR-NK vectors

B) The cytotoxicity results with CAR-NK cells and control primary NK cells. In K-562 cells, which lack the Her2 and MUC-Tn antigens, all the CAR-NK cells had equal killing capacity compared with untransduced primary NK cells. Using T-47D cells, which express both the Her2 and MUC-Tn antigens, the CMV- $\alpha-H e r 2-C A R-E F 1 a-G F P$ and EF1a- $\alpha-M U C 1-C A R$ induced higher cytotoxicity than the untransduced or GFP-only control transduced primary NK cells. On the other hand, CMVa-MUC1-CAR-EF1a-GFP were low Fab and protein $L$ positive, indicating a low a-MUC1-CAR expression also showed no enhancement in the killing assay.

Summary: The a-MUC1-CAR function correlates with the Fab and protein $L$ staining results. The higher the staining, the more CAR positive NK cells, the higher the cytotoxicity. Now, a maximum of $33 \%$ transduced cells was obtained, it can thus be expected that after optimizing transduction efficiencies, the cytotoxicity will increase further. This will be the main target in future experiments.

Limitations: Flow cytometry-based cytotoxicity detection method is an endpoint approach, which may not enough for dynamic monitor the killing capacity. 


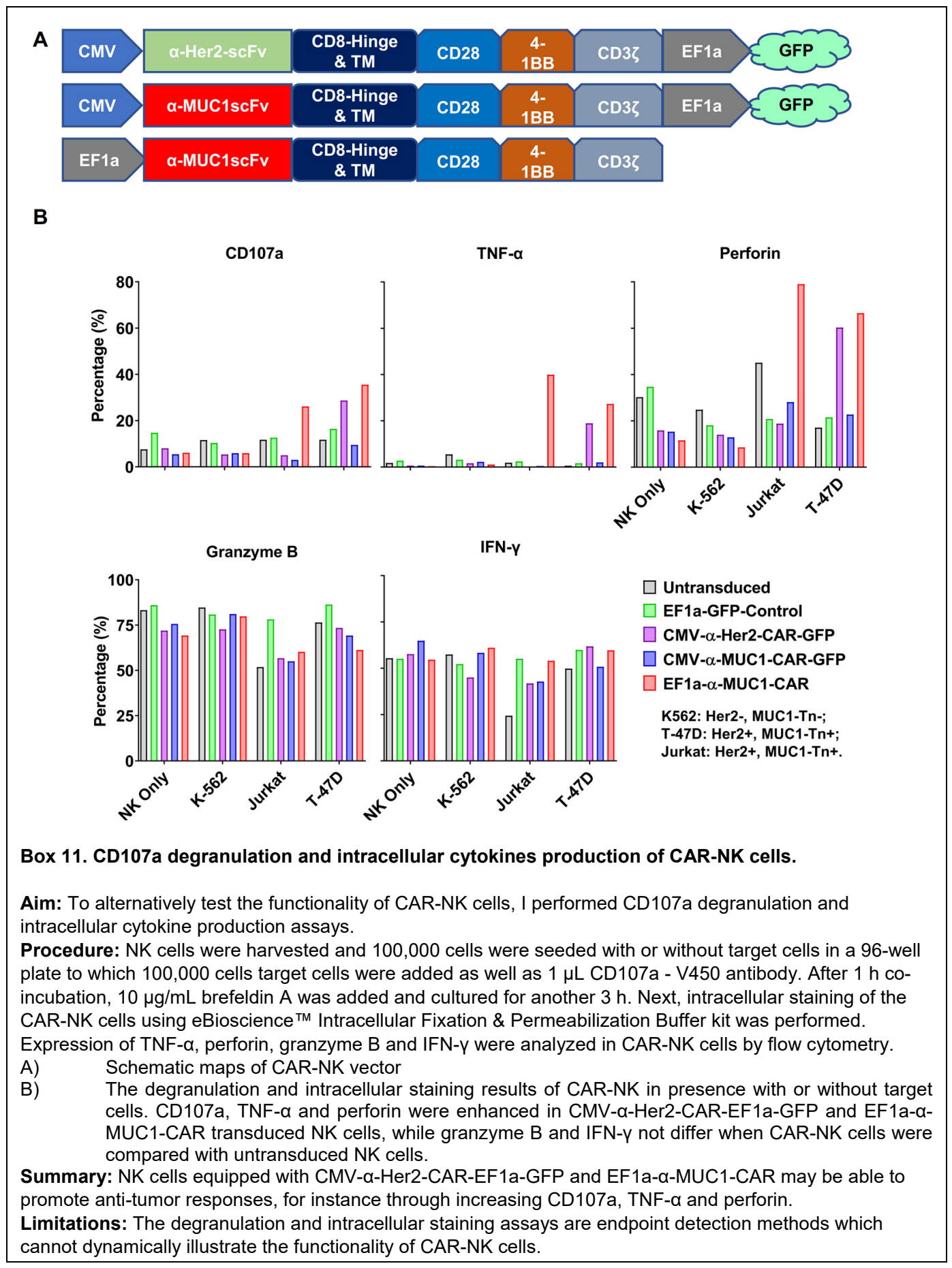




\section{Box 12. Conclusion of the boxes on the generation of CAR-NK cells plus recommendations for the future.}

During the past years I have explored the procedures to generate a functional CAR-NK. Initially, we designed $11 \mathrm{mCD} 19-\mathrm{CAR}$ constructs that aimed to determine the optimal intracellular activation signal for CAR-NK. However, the vectors contained the markers GFP and LNGFR to represent CAR expression on the NK cells but I hardly observed any enhancement in cytotoxicity with all 11 CARs. Detection of the CAR itself became the target of the next series of experiments. To that end, I cloned an anti-MUC1 scFV derived from the 5E5 humanized antibody described in Chapter 2 into a 4-1BB-DAP12 or 4-1BB-DAP10 containing vector, hypothesizing that NK cell specific intracellular domains would be optimal for CAR activity. As I demonstrated that GFP or LNGFR did not correlate with CAR expression, other detection methods were explored. CAR expression could be detected with Protein $L$ staining or a specific Fab fragment. Finally, I obtained preliminary results showing enhancement of cytotoxicity against $\mathrm{MUC1^{+ }}$ tumor cell lines using a MUC1-CAR with the CD28-4-1BB-CD3乙 activation signal.

In the future, I propose to pay more attention on the transduction efficiency that will lead to the generation of a more potent CAR-NK cell population and then try to compare the cytotoxicity of CAR-NK with antibody triggered ADCC. We should continue to use the safe lentivirus system to generate an anti-MUC1 CAR-NK and keep trying to optimize the design of the structure of the CAR-NK using the lessons learned in this review. The Fab antibody staining method to evaluate the CAR expression level should be continued. The current cytotoxicity and functional assays are useful assays to validate the CAR-NK, but a dynamic killing assay as can be measured on a live-cell culture analysis system (e.g. Incucyte) may useful as well to measure the kinetics of killing in real time.

All the virus work in this study is under the GMO bio-safety license: GGO-00-177 and GGO-17-006 under the supervision from department of CRISP bio-safety administration of Maastricht University. We thank Prof. dr. Reno Debets and Dr Cor Hj Lamers kindly provide the CAIX CAR retrovirus supernatant. 


\section{Chapter 5}

NKG2A genetic deletion promotes human NK cell antitumor responses by reversing HLA-E-mediated inhibition

Gong Y, Germeraad WTV, Janssen L, Gijsbers BLMG, Olieslagers TI, Bos GMJ, Klein Wolterink RGJ.

Submitted to Frontiers in Immunology 


\section{Chapter 6}

\section{General Discussion}


Cancer is considered one of the leading causes of mortality and also responsible for reducing life expectancy globally [1]. Conventional treatments like surgery, chemotherapy, and radiotherapy have been reported to be effective in eliminating tumor cells. Surgery is the method of choice when tumors are not metastasised. Next to that, Radio- and especially chemotherapy are found to possess low tumor specificity, thus cannot always discriminate between normal and malignant cells. This results in damage to healthy cells during therapy [2]. Moreover, the traditional therapies have been reported to possess poor efficacy in controlling metastasized tumors [3]. Therefore, novel therapies with enhanced tumor specificity and reduced adverse effects are urgently required for the effective management of malignant diseases.

Recently, cancer immunotherapy has gained importance as a promising anti-cancer therapy due to its impressive outcomes. The human immune system can be trained to target highly specific tumor cells and even the metastatic cancer cells (to the distal organs) without injuring or damaging healthy cells and tissues [4,5]. Technological advancements have facilitated this type of therapy to employ the human immune system to eradicate neoplastic cells specifically. Cancer immunotherapy has been broadly implemented in cancer treatment, and detailed information on this therapy has been introduced in chapter 1 . Adoptive cell therapies directed against tumors have achieved unprecedented success in patients with malignant disease with CAR-T cells at the forefront of success [6]. Moreover, natural killer (NK) cells are found to exhibit high cytolytic activity towards malignant cells (even without prior activation) and capable of alleviating side effects in patients [7]. Furthermore, the risk of graft versus host disease due to infusion of NK cells is low-to-none [7,8]. Therefore, NK cell-based immunotherapy has been regarded as an effective anti-cancer therapy and a possible alternative treatment to other conventional cancer therapies.

Until July 2021, the clinicaltrials.gov database recorded 540 clinical studies involving NK cells, by searching with "natural killer cell" as keywords. Among these studies, 288 clinical trials are found to be investigating the role of NK cells in treating cancers [9]. However, the overall response in cancer patients (especially with solid tumors) receiving NK cell infusion needs further improvement [10-12]. Certain critical aspects (such as the suppressive tumor microenvironment (TME) [13], reduced expression levels of NK cell activation receptors [14] and inadequate infiltration of NK cells in the tumor) are reported to be responsible for diminishing the outcome of NK cell activity $[7,9,15]$.

\section{Is the NK cell regimen solely effective for cancer immunotherapy?}

Our group has investigated NK cell therapy against multiple myeloma (MM) and breast cancer for two decades. We have found NK cells to be effective in eliminating multiple types of cancer cells in vitro and mouse models, but the efficiency of the NK cells in vivo needs to be studied further. About two decades ago, the first application of NK cell-based therapy had achieved an impressive anti-leukaemia response. This was due to the killer Ig-like receptors (KIRs) of donor NK cells that showed a mismatch with the recipient's human leukocyte antigen (HLA) class I alleles $[16,17]$. This mismatch between KIR and HLA further boosted the ability of the anti-tumor response of NK cells 
via the graft-versus-leukaemia (GVL) reaction. Recently, a phase I clinical trial using dose escalation has demonstrated high numbers of expanded NK cells on K562 feeder cells (expressing membrane-bound IL-21 and 4-1BBL) as safe with elevated tumor eradication in 13 patients with high-risk acute myeloid leukaemia (AML), myelodysplastic syndromes (MDSs) or chronic myeloid leukaemia (CML). Eleven out of 13 patients achieved remission after infusion of expanded NK plus hematopoietic stem cell transplantation (HSCT) [18]. Interestingly, a small prospective phase 2 study demonstrated that in chemo-resistant MM patients KIR-mismatch NK cell alloreactivity haploidentical stem cell transplantation (haploSCT) is not superior to conventional alloreactive stem cell transplantation (alloSCT) [19]. This variation might be due to the difference in the TME profiles of MM and AML that suppress the function of NK cells [20]. One study observed an upregulated HLA-E expression in MM cells in a murine model [20].

Although NK cell therapy might become effective in hematologic malignancies, the treatment of solid tumors is even more challenging. In a clinical trial on patients with recurrent paediatric medulloblastoma and ependymoma, they received the NK cell infusion through the lateral ventricle or fourth ventricle to bypass the blood-brain barrier. This renders the expanded NK cells to access the tumor site easily. However, an inadequate clinical response was observed in patients towards this NK cell therapy [12]. It has been demonstrated that the function of NK has been restrained by multiple factors in a TME [21]. These factors include stromal cell and tumor cell-induced hypoxia and surroundings with low $\mathrm{pH}$, secretion of a plethora of suppressive cytokines (such as TGF- $\beta$ ), and elements (such as PGE2, NO) by tumor cells [22]. These factors can dramatically reduce the infiltration of NK cells and their persistent functioning.

The clinical studies on adoptive NK cells where effector cells have been infused multiple times to maintain enough killer cells have demonstrated NK cell therapy as feasible and safe $[12,18]$. Based on the clinical outcome, encouraging remission has been observed in some haematological malignancies when treated solely with NK cell regimen. In contrast, $\mathrm{MM}$ and the majority of solid tumors with advanced suppressive TME have shown limited response. Fortunately, scientists and physicians have implemented combination therapy (with antibodies) to control neoplastic diseases.

\section{Antibody therapy endows a tumor antigen-specific receptor to NK cells engaged with CD16}

Antibodies have become a regular medication for cancer therapy due to their ability to recognize tumor antigens and simultaneously binding with the Fc receptors on the effector cells, thereby boosting the anti-tumor response. Currently, there are 43 FDAapproved marketed antibody-based cancer therapies. These therapies have shown impressive treatment outcomes both in hematologic and solid tumors [23]. NK cells can identify their target cells through a series of activation and inhibition receptors that are not antigen-specific receptors and are capable of binding to a broad range of ligands expressed on tumor cells. This property endows NK cells with the ability to recognize a wide spectrum of target cells [24]. Although derived from normal cells, the tumor cells 
usually express some tumor-associated antigens (TAA). The antibodies can bind with these TAAs simultaneously encountering the FcyRIIla (CD16a) on NK cells, giving the effector cells another and specific ability to lyse the tumor cells. This process is known as antibody-dependent cell-mediated cytotoxicity (ADCC) [8]. Besides inducing ADCC, the antibody can also trigger complement-dependent cytotoxicity (CDC) and antibodydependent cellular phagocytosis (ADCP) that help the human immune system to conduct tumor eradication [25].

We have investigated current commercial antibodies for $\mathrm{MM}$ for a better understanding of the therapy. At present, there are 3 FDA approved antibodies specific for MM therapy that includes elotuzumab (anti-SLAMF7/CS1) [25,26], daratumumab, and isatuximab targeting CD38 $[27,28]$. Although these three antibodies have been successfully implemented in clinical therapy and have achieved encouraging outcomes in MM patients (especially in the early stage of disease progression), the results in some advance or refractory/relapsed MM still need to be improved [29]. The inability in some MM patients to achieve any improvement of the disease may be attributed to three NK cell-related factors. Firstly, some NK cells either lack CD16 receptors or have downregulated CD16 receptors, and also, a particular polymorphism of CD16 (V158F) constrains the antibody binding with the NK cells [30]. Secondly, the CD38 and SLAMF7 antigens expressed on NK cells may induce NK cell-fratricide [27,31]. Lastly, in the TME of MM, the percentage of NK cells is decreased, and the NK cells are found to express a high level of checkpoint receptors like TIM3, TIGIT, and CD96, which indicate exhaustion of NK cells on encountering tumor cells [30]. These exhaustion markers are highly upregulated on activated effector NK and T cells, and they transmit the inhibitory signal that reduces the functional activities of the effector cells.

Another application of antibodies is to block these inhibitory signal transduction pathways by impeding the binding of the trafficking checkpoint receptors with their ligands [32-34]. These antibodies have dramatically unleashed the potential of NK cells or T cells in vivo and have demonstrated an improvement in the overall survival rate in multiple cancer types [35,36]. A clinical trial recently demonstrated that anti-PD1 plus allogeneic NK cells resulted in better survival benefits than anti-PD1 alone in advanced non-small-cell lung cancer patients [37]. Poznanski et al. showed that combined therapy of anti-PD1-blockade and expanded NK cells from lung cancer patients augment the cytotoxic ability of NK cells and provide strong antitumor activity against PDL1 positive and PDL1 negative tumors [38]. Thus, the checkpoint blockade antibodies can be considered as a combinational therapy with NK cells.

As mentioned, antibody treatment in cancer aims to endow the NK cells with an antigen-specific CD16-engaged receptor to redirect the target cells. To further augment the tumoricidal effect of this antibody-NK cell therapy, there are several combinational therapeutic approaches like chemotherapy combined with steroid conjugates and radiations [39]. The combination of the novel drugs, including proteasome inhibitors ( $\mathrm{PI})$ (such as carfilzomib and bortezomib) and immunomodulatory agents (such as pomalidomide, lenalidomide, and thalidomide), are predominantly found to be effective in MM patients [40]. Therefore, the antibodies plus NK cells still need further supplemental regimens in order to attain a satisfactory clinical outcome. 


\section{Augmentation of NK cell function through a disruption of the inhibitory signal}

NKG2A is an inhibitory receptor on NK cells and is considered a novel checkpoint inhibitory target. HLA-E, an MHC class I molecule expressed on tumor cells, is the ligand of NKG2A expressed on NK cells [41,42]. The TCGA cohort transcriptomes' data indicated that HLA-E was expressed at higher levels than PD-L1 in various cancer types [43]. Moreover, NKG2A is a dominant transcriptionally induced checkpoint important for cytokine-induced memory NK cells that may reduce the function of the effector cells in the TME $[44,45]$.

NKG2A is an important molecule responsible for the development and maturation of NK cells [46-49]. The NKG2A receptor and activated KIR molecules can recognize selfMHC class I proteins followed by binding and eventually killing normal healthy cells. Therefore, education of NK cells to distinguish "non-self" abnormal cells from "self" healthy cells is essential to inhibit NK-cell mediated autoimmunity (described in Chapter 1) [50]. NKG2A delivers a suppressive signal, which restrains the activities of NK cells. Antibody blockade and shRNA interference on NKG2A have proven to enhance the function of NK cells [51-53]. However, the anti-NKG2A antibody clinical trial demonstrated that the antibody blockade of NKG2A alone had a less pronounced tumoricidal effect than its use in combination with anti-PD-L1 and/or anti-EGFR antibodies [51]. Although the use of the anti-NKG2A antibody has shown enhancement of the anti-tumor activity of NK cells and T cells, the efficiency of this antibody was found to be limited [51]. Interestingly, a novel approach involving intracellular targeting of NKG2A to retain the protein in the endoplasmic reticulum has exerted stronger blockade efficiency than anti-NKG2A antibody both in vitro and in a mouse model [52].

We hypothesized that ablation of the NKG2A receptor gene may exert better blockade efficiency than the antibody blockade. In chapter $\mathbf{5}$, we have described the generation of NKG2A knockout human primary NK cells by applying CRISPR/Cas9 technology. We observed no significant differences in the transcriptome between NKG2A knockout and wild-type (WT) human primary NK cells. Moreover, the expression level of phenotypic and functional molecules has not changed. Interestingly, the NKG2A ${ }^{\mathrm{KO}}$ NK cells can overcome the inhibitory signal from high HLA-E expressing tumor cells. Thus, NKG2A ${ }^{\mathrm{KO}}$ may improve the persistence of NK cells towards a high HLA-E expressing tumor suppressive microenvironment. Further experiments to verify the safety of NKG2AKO NK cells in clinical trials are needed [20]. It will be important to conduct a study in a xenograft mice model to validate our hypothesis further. Furthermore, it will be interesting to compare the siRNA or shRNA silencing, antibody blockade, and CRISPR/Cas9 mediated gene knockout. We know that these three approaches can down-regulate gene expression or block the signal transduction pathway; however, comparing the blockade efficiency between these three methods would be important research based on the current observations that differences do exist. One of the major concerns of the CRISPR/Cas9 mediated gene modification of the immune cell is safety since engineered cells may induce severe adverse effects after infusion. Fortunately, the first-in-human, phase I clinical trial on CRISPR/Cas9 engineered $T$ cell therapy has demonstrated multiple gene-modified $T$ cells as safe and feasible in advanced refractory tumor patients [54]. In this study, PDCD1 (encoding PD1), 
TRAC (encoding TCR alpha), and TRBC (encoding TCR beta) have been successfully knocked out in T cells, and simultaneous lentivirus transduction of the anti-NY-ESO-1 cancer antigen-specific was conducted in the same T cells [54]. Removal of the endogenous TCR to reduce mispairing of TCR-chains has been reported to induce severe adverse effects [55,56], while disruption of the PD1 gene is aimed to improve the persistence of T cells in vivo [57]. Interestingly, the infusions with NY-ESO-1 TCR CRISPR/Cas9 T cells in this study were well tolerated with no serious adverse events and no cases of cytokine release syndrome (CRS), which is a potentially lethal adverse effect of cellular immunotherapy $[54,58]$. Thus, we believe that CRISPR/Cas9 modified NK cells can be considered safe and potentially feasible for cancer treatment.

Besides, there are other inhibitory receptors on NK cells transmitting suppressive signals, which could be alternative candidates possible to boost functional activities. Two studies have illustrated that CRISPR/Cas9 mediated knockout of cytokine-inducible Src homology 2-containing (CIS) protein (encoded by $\mathrm{CISH}$ gene) can boost the cytotoxicity and aerobic glycolysis of NK cells $[59,60]$. Furthermore, the checkpoint inhibitors (like TIM3 and TIGIT) are abundantly expressed on human mature NK cells, which deliver the suppressive signals that inhibit the functioning of the NK cells. In a glioma cell model, the tumor lytic abilities of TIM3 ${ }^{K O}$ NK cells outperformed TIM3 expressing NK cells. Interestingly, in this study, the growth inhibitory effect of exon $2 \mathrm{TIM}^{\mathrm{KO}}$ surplus exon 5 TIM3 $^{\mathrm{KO}}$ NK cells, due to increased expression of TIM3 molecules in exon 5 TIM3 ${ }^{\mathrm{KO}}$ NK cells [61]. Another study used CRISPR/Cas9 mediated ablation technology to target the Casitas B-lineage lymphoma pro-oncogene-b (CBLB), a ubiquitin ligase, and found that NK cells without $C B L B$ exhibited enhanced cytotoxicity on MM and leukaemia cells both in vitro and in a murine xenograft model [62]. Similarly, Cas9-mediated knockout of $A D A M 17$, a disintegrin and metalloprotease would cleave CD16a molecules after activation of NK cells by an antibody in human NK cells, further augmented ADCC $[63,64]$. Above all, numerous attempts have been made to boost the cytotoxicity of NK cells via reducing the inhibitory signal on NK cells, which can be further utilized clinically to treat cancer.

\section{MUC1-Tn is still a promising target for solid cancer}

The target epitope of any molecule of an antibody will determine the efficacy and outcome of the immunotherapy treatment. An ideal tumor antigen target should be abundantly expressed on cancerous cells while rarely presented on normal healthy cells. Mucin1 (MUC1), a tumor-associated antigen, is a heavily O-glycosylated membrane glycoprotein, which is expressed at a low level on the normal epithelial tissue $[65,66]$. The core element of MUC1 consists of variable number tandem repeats (VNTR) of 20 amino acids (AHGVTSAPDTRPAPGSTAPP). There are 5 available glycosylation sites in the repeats, mainly on the serine and tyrosine residues [67].

The MUC1 protein is partially glycosylated (or heavily underglycosylated) and overexpressed in tumor cells, facilitating the discrimination of healthy $\mathrm{MUC}^{+}$cells from MUC1 overexpressing cells in cancer patients. This is associated with a poor prognosis, making MUC1 a promising target for tumor immunotherapy [67-69]. Our group has previously tested six anti-MUC1 antibodies (214D4, HMFG-1, VU4H5, SM3, 1B9, and 
5E5) by staining the MUC1 expression on bone marrow (BM) of immature and mature plasma cells. Surprisingly, we found that only four out of 19 MM patients' BM cells are positive for the 5E5 antibody. Thus, the MUC1-Tn epitope might not be an ideal tumor target for MM [70].

MUC1-Tn might be barely expressed on hematologic tumors, but is extensively expressed on solid tumors. Previous studies found $>90 \%$ of breast cancer biopsies to be MUC1-positive [71] and our group has found that all most $100 \%$ of ovarian cancer is underglycosylated MUC1 positive [72]. Previously, several antibodies against MUC1 have been applied in clinical trials, but these studies lack promising clinical outcomes even when combined with an aromatase inhibitor, which have been proven to enhance the ADCC of anti-MUC1 therapy [73-76]. Moreover, a study reported anti-MUC1 antibody HMFG1 mediated ADCC can be inhibited by the high level of MUC1 in the serum derived from ovarian and breast cancer patients [77]. Additionally, the HMFG-1 antibody can be internalized in the cytoplasm of tumor cells within 15 min that may hamper the ADCC [78]. A phase II clinical trial conducted on breast tumor patients using another anti-MUC1 antibody, AS 1402, have reported that no encouraging outcomes after receiving the antibody therapy [73]. Novel antibodies targeting MUC1 are still burgeoning to improve the efficiency of MUC1 targeting therapy.

The murine 5E5 targeting MUC1-Tn epitope antibody has exerted high binding affinity and can trigger ADCC towards MUC-Tn positive tumor cells in vitro [79,80]. Murine 5E5 antibody showed potent tumoricidal activities toward pancreatic and T cell lymphoma cells in a mice model [81]. Notably, these 5E5 antibodies have no cross-reaction with activated T cells [81]. In chapter 2, we continued our previous research on antibodies targeted towards MUC1. We humanized the 5E5 anti-MUC1-Tn antibody to explore the possibility of applying it in combination with human NK cells to conquer MUC1-Tn positive tumor cells. Our results showed that the humanized 5E5 antibody (both CIM3011 and CIM301-8) can activate NK cells and trigger ADCC. Moreover, the defucosylation on Fc fragments (CIM301-8) can further augment the ADCC. Surprisingly, we observed a reduction in the level of CD16 on NK cells on increasing the antibody dose, and the endocytosis inhibitors could not further enhance the ADCC (Chapter 2). In the next step, we aim to further verify whether our humanized 5E5 can efficiently work in a xenograft mice model. The work presented in this chapter is still encouraging to continue to investigate MUC1 as a target using immunotherapy for cancer.

\section{Ideal NK cell source for cancer immunotherapy}

NK cells are achieving great interest in cancer immunotherapy; however, the source of NK cells will dramatically influence the quality of the effector cells. The presence of heterogeneous NK cells can pose challenges in selecting the appropriate NK cells for immunotherapy [8]. NK cells, initially characterized as potent cytotoxic killer cells, have shown an unexpected cell complexity (both phenotypic and functional aspects) with the help of novel techniques (e.g., mass spectrometry, single-cell sequencing technology, and flow cytometry) [82-87]. A study estimated about 6,000 to 30,000 phenotypic NK cell sub-populations to be present in individual human adult peripheral blood with the help of mass spectrometry [85]. This diversity in the NK cell population could be due to 
antiviral responses by the human body during early life stages, which indicates the immune experience and correlates with the viral susceptibility [88]. Despite the fact that NK cells are complex in the human body, mature NK cells in peripheral blood are capable of killing target cells after they received properly activation signals [88].

There are several sources for NK cell adoptive therapy, such as peripheral blood NK cells from allogeneic donors, umbilical cord blood NK cells, and stem cell-differentiated NK cells. Different sources of NK cells manifest dissimilar phenotypes and functions. Peripheral blood-derived CD19 CAR-NK cells possess a slightly stronger anti-B cell tumor response, while CD19 CAR-NK cells engineered from cord blood are more potent in expansion and susceptible to cytokine stimulation [89]. The reason for the difference could be due to the experience of the peripheral blood NK cells interacting with antigens, while cord blood NK cells lack such processes. However, the degranulation ability shows no difference in these two sources of NK cells. In addition, human embryonic stem cell (hESC) differentiated NK cells possess a higher killing capacity than cord blood-derived NK cells since hESC-derived NK cells are uniformly CD94 ${ }^{+}$CD117low/- [90]. Moreover, CD34+ umbilical cord blood (UCB) derived NK cells have a phenotype expressing similar levels compared with CD56+ UCB NK cells when obtained from the same donor [91]. However, the CD2, CD16, and KIR receptors (KIR2DL3 and KIR2DL1) are expressed higher on the CD56+UCB derived NK cells and these molecules have been demonstrated to be associated with the NK cell differentiation stage. Interestingly, CD56+ NK cells directly isolated (and expanded) from UC have consistently better cytotoxic activity than CD34+ derived UCB NK [91]. This study also described the cytotoxicity difference between KIR positive and KIR negative iPSC derived NK cells. KIR+ iPSC and KIR- iPSC derived NK cells expressed similar levels of activating receptors, including FasL, TRAIL, NKp44, NKp46, and NKG2D, but the CD16 expression is higher in KIR+ iPSC derived NK cells [91]. Surprisingly, there is no significant difference of cytotoxicity between KIR+ iPSC and KIR- iPSC derived NK cells against K562 target cells [91]. NK cell lines have also proven their safety in several clinical trials. Adoptive NK-92 cells, one of the most common used NK leukaemia cell line, have been infused in both lymphoma and solid tumor patients, like breast cancer, glioma, gastric carcinoma [92]. Thus, various NK cells source may exert different functionality.

iPSC-derived NK cells can be utilized as an important NK cell source because this method can generate uniform effector cells to avoid the heterogeneity of mature NK cells in the peripheral blood or cord blood. In addition, the iPSC-derived NK cells can undergo clonal expansion even after genetic modifications. However, the main limitation of iPSCderived NK cells is the differentiation procedure, which is laborious, challenging and expensive. Furthermore, it remains to be established whether these cells will be subject to a host vs graft reaction in patients. Thus, multiple sources of NK cells can be utilized for cancer immunotherapy.

\section{CAR-NK is becoming an "Off-the-shelf" cell product for cancer immunotherapy}

NK cells are potent killer cells, and their tumor specific cytotoxicity can be further enhanced by genetic modification with a chimeric antigen receptor (CAR). The CAR 
comprises three parts, which include an antibody-based receptor on the cell surface, a transmembrane domain anchoring on the membrane, and an intracellular domain (Chapter 1 and Chapter 4). The first clinical trial on primary CAR-NK cells has revealed promising results for CD19-positive B cell malignancies and did not indicate severe adverse effects (e.g., cytokines release storm (CRS) and immune cell-associated neurotoxicity syndrome (ICANS) that are often reported in CAR-T clinical trials) [93]. This CD19-CAR-NK study demonstrated that effector cells could persist for more than one year. However, in this study, CAR-NK cells were traced by a CAR gene PCR test instead of flow cytometry or other NK cell-specific marker staining methods (discussed in Chapter 4) [93].

In Chapter 4, we reviewed the state-of-the-art design and engineering of CAR-NK. We elaborated every CAR construct and performed statistical analysis of the CAR designing, gene-transfer approaches, detection methods, and NK source from the currently published valuable studies. In the meantime, we summarized several key progressions while we attempted generating our 5E5 anti-MUC1-Tn CAR-NK. These preliminary data can help in achieving a more straightforward understanding of the difficult procedures to successfully generate CAR-NK. Moreover, the murine 5E5 antiMUC1antibody based CAR-T has shown impressive results in eradicating $T$ cell lymphoma and pancreatic cancer cells in xenograft mouse models [81]. These 5E5 engineered CAR-T lack the ability to self-react with human T cells when activated T cells express healthy MUC1 [81]. Recently, an in vivo study has reported that the CRS is alleviated in CAR-NK infused mice compared to CAR-T cells [94]. Thus, our humanized 5E5 anti-MUC1 CAR-NK could be able to display a strong ability to kill tumor cells while being safe on tumor patients. In the next step, several direct cytotoxicity assays will be conducted to evaluate and compare the enhancement of antibody binding capacity of NK cells and CAR-NK cells.

Inspired by the encouraging data from murine 5E5 CAR-T by Posey et al. [81], we planned to generate humanized 5E5 CAR-NK for tumor immunotherapy. Regarding the impressive results from 5E5 CAR-T and our humanized antibody study (Chapter 2), we hypothesize that MUC1-Tn specifically expressed on solid tumor cells is a good target. The murine 5E5 CAR-T may induce a host versus graft disease as the scFv is derived from a mouse antibody [81]. In Chapter 4, we elaborate on our road to generate a humanized 5E5 CAR-NK. Although we came across many obstacles, we encouragingly observed once an enhanced killing capacity with the MUC-Tn-CAR-NK. Therefore, we still consider the humanized MUC1-Tn CAR-NK as potentially powerful effector cells to conquer MUC-Tn positive cells.

Besides generating CAR-NK, the humanized 5E5 antibody can be further applied with other antibodies as a combination therapy. Our data showed the level of CD16 on NK cells reduced after binding with CIM301-8; thus, the ADAM17 inhibitor may rescue the dis-regulation of CD16 molecules. Moreover, checkpoint blockade antibodies may also facilitate the CIM301-8 antibody to achieve a better outcome. In our future experiments, this CIM301-8 antibody can also be utilized to generate a TriKE [8,95], which contains the 5E5 scFv, IL-15, and anti-CD16 that may further enhance the efficiency of NK cells to eradicate MUC1-Tn epitope positive tumor cells in vivo. 


\section{Boosting lentiviral transduction efficiency via upregulating viral receptor expression on NK cells}

To generate CAR-NK, the most commonly used vehicle to introduce a CAR is viral transduction. Unfortunately, NK cells are resistant to viral infections which may be the reason for their transduction difficulty $[96,97]$. The VSV-G enveloped lentivirus is widely used in gene therapy. LDLR has been demonstrated as the receptor for the VSV-G virus $[98,99]$. In Chapter 3, we screened 5 statins and 5 non-statins to test whether these compounds can boost the expression of LDLR on NK cells and thereby enhancing lentiviral gene transfer. The screened 5 non-statin compounds included IL-21 cytokine [100], ascorbic acid (Vitamin C) [101], dextran [102], prostaglandin E2 [103], and vectofusin-1 [104]. They were chosen from previous studies that have been reported to enhance virus transduction efficiency on human immune cells. We observed that statins boost the expression of LDLR receptor both on primary NK cells and NK-92 cells and were correlated with the GFP positive population when transduced with a GFP coding VSV-G lentivirus. Unfortunately, statins have been reported to impair the function of NK cells [105-107]. When the geranylgeranyl pyrophosphate (GGPP), a molecule part of the statin metabolism pathway, was added in the NK cell culture, the cytotoxic functions of NK cells were completely reversed. Thus, we believe that the statins (especially rosuvastatin) plus GGPP may serve as an alternative pathway to boost lentiviral transduction, facilitating CAR-NK generation for tumor immunotherapy.

Although we demonstrated that lentiviral transduced NK cells are correlated with LDLR receptor expression both on NK-92 and primary NK cells, other receptors (e.g., VLDLR, LRP1 LRP2, LRP8, and MEGF7) have been reported to serve as the receptors for VSV-G lentivirus $[98,108]$. These alternative VSV-G receptors may guide future research to find more potent candidates that can boost the VSV-G mediated transduction efficiency. Moreover, other pseudo-type viruses may facilitate NK cell transduction efficiency. Thus, boosting the LDLR receptor on NK cells is not the only approach to increase the transduction efficiency of NK cells. In the future, more potent compounds will be developed to ensure improved lentiviral transduction on human NK cells. Optimization of the lentiviral-mediated transduction efficiency on NK cells will pave the way to generate CAR-NK.

Besides viral transduction, alternative approaches have been applied to generate CAR-NK. Li et al. used a transposon to introduce the CAR gene into iPSC cells and then differentiated them into CAR-NK cells, generating homogeneous effector cells [94]. In Chapter 4, we introduced the CRISPR-mediated CAR introduction, which may be considered as an alternative method [109]. With the CRISPR/Cas9 system, a gene can be targeted to integrate into the desired gene locus, thereby circumventing the viral gene promoter silencing after transduction and the risk of leukemogenesis [110,111]. Moreover, CAR mRNA could be electroporated into human NK cells, and a robust antitumor response mediated by CAR can be generated [112]. The advantages and disadvantages of these CAR gene delivery methods are summarized in Chapter 4. 


\section{Clinical applications and social impact of CAR-NK therapy}

With the advancement of CAR-T technology, CAR-modified T cells have achieved great progress in cancer immunotherapy. In 2017, the FDA approved two CAR-T cell therapies for diffuse large B-cell lymphoma: axicabtagene ciloleucel (axicel) (priced $\$ 373,000$ ) and tisagenlecleucel (priced $\$ 475,000$ ) $[113,114]$. However, CAR-T therapy requires additional supplemental drugs to reduce its adverse effects and assure its efficiency. Hence, the overall price of each treatment may increase up to \$1 million [115]. Despite the effectiveness of these novel therapies leading to a prolonged survival period of malignant patients, the economic burden associated with these therapies is regarded as the primary constrain for their worldwide implementation [116]. Technological advancements might reduce the cost of CAR-T by around $30 \%$ by 2030 [116].

Considering the substantial economic burden, alternative cheaper therapies are urgently needed in clinical practice. NK cells without severe adverse effects and being less expensive can be utilized as an alternative therapy for tumor patients. The development of GMP compliant, large scale expansion systems for NK cell production can assure the application of NK cells as affordable "living drugs" even in developing countries.

We have discussed the different sources of NK cells in generating CAR-NK (Chapter 4). From this, one can hypothesize that an ideal candidate NK effector cell should possess several of the following:

i) highly specific scFv (Chapter 2),

ii) high expression levels of activating receptors like NKG2D, natural killer cell receptors (NCR) and cytokine receptors,

iii) reduced or no expression of inhibitory receptors, like NKG2A, PD-1, TIGIT, and/or inhibitory KIR receptors (Chapter 5).

iv) in case of lentiviral vectors: high LDLR expression levels to facilitate VSV-G virus transduction (Chapter 3),

Such NK cells can be implemented as an off-the-shelf product, activated by the tumor antigens through the CAR and tolerable under the immunosuppressive tumor microenvironment.

To make an "ideal NK cell" a realistic "off-the-shelf" cell product for tumor immunotherapy, one can hypothesize that iPSC differentiated NK might be a good option after further genetic manipulation.

Several questions need to be answered before our community obtains the ideal NK cell. Some of these outstanding questions are:

i) how to efficiently introduce CAR genes into NK cells?

ii) how to make NK cells persist and active in a suppressive tumor microenvironment?

iii) does downregulation of inhibitory receptors like NKG2A or KIR on NK cells render NK cells to lose the ability to identify "self-antigens" from "non-selfantigens," required for leaving healthy tissue untouched?

iv) How can donor NK cells survive host immune responses against them? 


\section{4 | Chapter 6}

Still, expanded NK cells, obtained from peripheral blood in combination with other therapies (antibodies/checkpoint inhibitors) either or not genetically modified and used in a clinical donor bone marrow transplantation setting might be the optimal way to explore the clinical opportunities of NK cells.

Overall, in the present thesis, our data indicated various approaches to enhance the functions of NK cells used for tumor immunotherapy, which can be considered a safe and effective treatment of cancer. Future clinical trials will validate these findings. 
Table 6.1 Main findings, limitations and applications of each Chapter.

\begin{tabular}{|c|c|c|c|}
\hline Chapters & Main findings & $\begin{array}{l}\text { Limitations and } \\
\text { next steps }\end{array}$ & Applications \\
\hline Chapter 2 & $\begin{array}{l}\text { Successfully Humanized 5E5 anti- } \\
\text { MUC1-Tn antibody (CIM301-1 and } \\
\text { 8); } \\
\text { CIM301-1 and } 8 \text { antibodies } \\
\text { recognize the MUC1-Tn on tumor } \\
\text { cells and bind CD16 on NK cells; } \\
\text { CIM301-1 and } 8 \text { antibodies activate } \\
\text { NK cells and trigger ADCC; } \\
\text { CIM301-8 with defucosylated Fc } \\
\text { further enhances the cytotoxicity of } \\
\text { NK cells. }\end{array}$ & $\begin{array}{l}\text { More cancer cell } \\
\text { types need to be } \\
\text { verified with } \\
\text { CIM301-1 and } 8 \\
\text { antibodies; } \\
\text { In vivo models to } \\
\text { validate functional } \\
\text { activities of } \\
\text { CIM301-1 and } 8 \\
\text { antibodies. }\end{array}$ & $\begin{array}{l}\text { The Fc defucosylation of } \\
\text { antibodies further boosts the } \\
\text { anti-tumor functionality when } \\
\text { combined with NK cells; } \\
\text { CIM301-1 scFv can be used } \\
\text { to generate anti-MUC1 CAR- } \\
\text { NK. }\end{array}$ \\
\hline Chapter 3 & $\begin{array}{l}\text { LDLR receptor expression level on } \\
\text { NK cells was correlated with VSV-G } \\
\text { transduction efficiency; } \\
\text { Rosuvastatin plus GGPP can } \\
\text { improve the VSV-G transduction } \\
\text { efficiency on NK cells and facilitate } \\
\text { viral gene integration into the NK } \\
\text { cell genome. }\end{array}$ & $\begin{array}{l}\text { Statins will inhibit } \\
\text { the function of NK } \\
\text { cells, GGPP can } \\
\text { reverse the } \\
\text { suppression. }\end{array}$ & $\begin{array}{l}\text { Rosuvastatin plus GGPP can } \\
\text { boost the VSV-G lentivirus } \\
\text { transduction that can be } \\
\text { further applied in NK cell } \\
\text { genetic modification. }\end{array}$ \\
\hline Chapter 4 & $\begin{array}{l}\text { We reviewed the "state-of-the-art" } \\
\text { CAR-NK progress from the } \\
\text { published literature and clinical } \\
\text { trials; } \\
\text { We showed the current stage for } \\
\text { generating anti-MUC1 CAR-NK in } \\
\text { our lab; }\end{array}$ & $\begin{array}{l}\text { As a model, NK-92 } \\
\text { will be transduced } \\
\text { with an anti-MUC1 } \\
\text { CAR, and FACS } \\
\text { sorting will be used } \\
\text { to make a CAR } \\
\text { stable expressing } \\
\text { cell line. }\end{array}$ & $\begin{array}{l}\text { Anti-MUC1 CAR-NK will be } \\
\text { generated in primary NK cells } \\
\text { and will be used in clinical } \\
\text { trials in the future. }\end{array}$ \\
\hline Chapter 5 & $\begin{array}{l}\text { Successfully knockout NKG2A in } \\
\text { expanded human primary NK cells } \\
\text { by CRISPR/Cas9; } \\
\text { NKG2A KO didn't alter the } \\
\text { transcriptome, phenotype, and } \\
\text { functional molecules in human NK } \\
\text { cells; } \\
\text { NKG2A KO cells overcome the } \\
\text { inhibitory signal from high HLA-E } \\
\text { expressing tumor cells, which } \\
\text { outperform the NKG2A antibody } \\
\text { blockade. }\end{array}$ & $\begin{array}{l}\text { Dynamic } \\
\text { cytotoxicity assays } \\
\text { with Incucyte } \\
\text { equipment may } \\
\text { help to understand } \\
\text { the cytotoxic } \\
\text { function of NKG2A } \\
\text { KO cells. } \\
\text { In vivo validation. }\end{array}$ & $\begin{array}{l}\text { The genetic knockout of } \\
\text { NKG2A may help NK cells } \\
\text { overcome the inhibitory tumor } \\
\text { microenvironment, which can } \\
\text { make NK cells more } \\
\text { persistent under TME } \\
\text { surroundings. }\end{array}$ \\
\hline
\end{tabular}




\section{References}

1. Sung H, Ferlay J, Siegel RL, Laversanne M, Soerjomataram I, Jemal A, et al. Global cancer statistics 2020: Globocan estimates of incidence and mortality worldwide for 36 cancers in 185 countries. CA Cancer J Clin. 2021. doi:10.3322/caac.21660.

2. Waldman AD, Fritz JM, Lenardo MJ. A guide to cancer immunotherapy: From $t$ cell basic science to clinical practice. Nat Rev Immunol. 2020; 20:651-668. doi:10.1038/s41577-020-0306-5.

3. Andrea N, Vivian B, Piermario B, Paola F, Giuseppe R, Angelo C. The use of immunotherapy to treat metastatic breast cancer. Current Medicinal Chemistry. 2019; 26:941-962. doi:http://dx.doi.org/10.2174/0929867325666180209124052.

4. Dianat-Moghadam H, Mahari A, Heidarifard M, Parnianfard N, Pourmousavi-Kh L, Rahbarghazi R, et al. Nk cells-directed therapies target circulating tumor cells and metastasis. Cancer Lett. 2021; 497:41-53. doi:https://doi.org/10.1016/j.canlet.2020.09.021.

5. Lorenzo-Herrero S, López-Soto A, Sordo-Bahamonde C, Gonzalez-Rodriguez AP, Vitale M, Gonzalez S. Nk cell-based immunotherapy in cancer metastasis. Cancers (Basel). 2018; 11:29

6. Demaria O, Cornen S, Daëron M, Morel Y, Medzhitov R, Vivier E. Harnessing innate immunity in cancer therapy. Nature. 2019; 574:45-56. doi:10.1038/s41586-019-1593-5.

7. Mujal AM, Delconte RB, Sun JC. Natural killer cells: From innate to adaptive features. Annu Rev Immunol. 2021; 39:417-447. doi:10.1146/annurev-immunol-101819-074948.

8. Myers JA, Miller JS. Exploring the nk cell platform for cancer immunotherapy. Nat Rev Clin Oncol. 2020. doi:10.1038/s41571-020-0426-7.

9. Kim KS, Kim DH, Kim DH. Recent advances to augment nk cell cancer immunotherapy using nanoparticles. Pharmaceutics. 2021; 13. doi:10.3390/pharmaceutics13040525.

10. Shaver KA, Croom-Perez TJ, Copik AJ. Natural killer cells: The linchpin for successful cancer immunotherapy. Front Immunol. 2021; 12:679117. doi:10.3389/fimmu.2021.679117.

11. Lamb MG, Rangarajan HG, Tullius BP, Lee DA. Natural killer cell therapy for hematologic malignancies: Successes, challenges, and the future. Stem Cell Res Ther. 2021; 12:211. doi:10.1186/s13287-021-02277-x.

12. Khatua S, Cooper LJN, Sandberg DI, Ketonen L, Johnson JM, Rytting ME, et al. Phase i study of intraventricular infusions of autologous ex vivo expanded nk cells in children with recurrent medulloblastoma and ependymoma. Neuro Oncol. 2020; 22:1214-1225. doi:10.1093/neuonc/noaa047.

13. Melaiu O, Lucarini V, Cifaldi L, Fruci D. Influence of the tumor microenvironment on nk cell function in solid tumors. Front Immunol. 2020; 10:3038. doi:10.3389/fimmu.2019.03038.

14. Parkhurst MR, Riley JP, Dudley ME, Rosenberg SA. Adoptive transfer of autologous natural killer cells leads to high levels of circulating natural killer cells but does not mediate tumor regression. Clin Cancer Res. 2011; 17:6287-6297. doi:10.1158/1078-0432.ccr-11-1347.

15. Melaiu O, Lucarini V, Cifaldi L, Fruci D. Influence of the tumor microenvironment on nk cell function in solid tumors. Front Immunol. 2019; 10:3038. doi:10.3389/fimmu.2019.03038.

16. Ruggeri L, Capanni M, Urbani E, Perruccio K, Shlomchik WD, Tosti A, et al. Effectiveness of donor natural killer cell alloreactivity in mismatched hematopoietic transplants. Science. 2002; 295:20972100. doi:10.1126/science.1068440.

17. Miller JS, Soignier Y, Panoskaltsis-Mortari A, McNearney SA, Yun GH, Fautsch SK, et al. Successful adoptive transfer and in vivo expansion of human haploidentical nk cells in patients with cancer. Blood. 2005; 105:3051-3057. doi:10.1182/blood-2004-07-2974.

18. Ciurea SO, Schafer JR, Bassett R, Denman CJ, Cao K, Willis D, et al. Phase 1 clinical trial using mbil21 ex vivo-expanded donor-derived nk cells after haploidentical transplantation. Blood. 2017; 130:1857-1868. doi:10.1182/blood-2017-05-785659.

19. Van Elssen C, van Gorkom G, Voorter C, von dem Borne P, Meijer E, Wieten L, et al. Haploidentical transplantation in patients with multiple myeloma making use of natural killer cell alloreactive donors. Annals of Hematology. 2020. doi:10.1007/s00277-020-04303-z.

20. Sarkar S, van Gelder M, Noort W, Xu Y, Rouschop KM, Groen R, et al. Optimal selection of natural killer cells to kill myeloma: The role of hla-e and nkg2a. Cancer Immunol Immunother. 2015; 64:951963. doi:10.1007/s00262-015-1694-4.

21. Binnewies M, Roberts EW, Kersten K, Chan V, Fearon DF, Merad M, et al. Understanding the tumor immune microenvironment (time) for effective therapy. Nat Med. 2018; 24:541-550. doi:10.1038/s41591-018-0014-x. 
22. Sordo-Bahamonde C, Vitale M, Lorenzo-Herrero S, López-Soto A, Gonzalez S. Mechanisms of resistance to nk cell immunotherapy. Cancers (Basel). 2020; 12:893. doi:10.3390/cancers12040893.

23. Goydel RS, Rader C. Antibody-based cancer therapy. Oncogene. 2021; 40:3655-3664. doi:10.1038/s41388-021-01811-8.

24. Guillerey C, Huntington ND, Smyth MJ. Targeting natural killer cells in cancer immunotherapy. Nat Immunol. 2016; 17:1025. doi:10.1038/ni.3518.

25. Shah UA, Mailankody S. Emerging immunotherapies in multiple myeloma. Bmj. 2020; 370:m3176. doi:10.1136/bmj.m3176.

26. Lonial S, Dimopoulos M, Palumbo A, White D, Grosicki S, Spicka I, et al. Elotuzumab therapy for relapsed or refractory multiple myeloma. N Engl J Med. 2015; 373:621-631. doi:10.1056/NEJMoa1505654.

27. Martin TG, Corzo K, Chiron M, Velde HV, Abbadessa G, Campana F, et al. Therapeutic opportunities with pharmacological inhibition of cd38 with isatuximab. Cells. 2019; 8. doi:10.3390/cells8121522.

28. Gauthier M, Laroye C, Bensoussan D, Boura C, Decot V. Natural killer cells and monoclonal antibodies: Two partners for successful antibody dependent cytotoxicity against tumor cells. Critical $\begin{array}{llll}\text { Reviews } & \text { in } & \text { Oncology/Hematology. } & \text { 2021; }\end{array}$ doi:https://doi.org/10.1016/j.critrevonc.2021.103261.

29. Minnie SA, Hill GR. Immunotherapy of multiple myeloma. J Clin Invest. 2020; 130:1565-1575. doi:10.1172/jci129205.

30. Pazina T, MacFarlane AWt, Bernabei L, Dulaimi E, Kotcher R, Yam C, et al. Alterations of nk cell phenotype in the disease course of multiple myeloma. Cancers (Basel). 2021; 13:226. doi:10.3390/cancers13020226.

31. Mahaweni NM, Bos GMJ, Mitsiades CS, Tilanus MGJ, Wieten L. Daratumumab augments alloreactive natural killer cell cytotoxicity towards cd38+ multiple myeloma cell lines in a biochemical context mimicking tumour microenvironment conditions. Cancer Immunol Immunother. 2018; 67:861-872. doi:10.1007/s00262-018-2140-1.

32. Zhang C, Liu Y. Targeting nk cell checkpoint receptors or molecules for cancer immunotherapy. Front Immunol. 2020; 11:1295. doi:10.3389/fimmu.2020.01295.

33. Khan M, Arooj S, Wang H. Nk cell-based immune checkpoint inhibition. Front Immunol. 2020; $11: 167$. doi:10.3389/fimmu.2020.00167.

34. Bi J, Tian Z. Nk cell dysfunction and checkpoint immunotherapy. Front Immunol. 2019; $10: 1999$. doi:10.3389/fimmu.2019.01999.

35. Darvin P, Toor SM, Sasidharan Nair V, Elkord E. Immune checkpoint inhibitors: Recent progress and potential biomarkers. Exp Mol Med. 2018; 50:1-11. doi:10.1038/s12276-018-0191-1.

36. Gubin MM, Zhang X, Schuster H, Caron E, Ward JP, Noguchi T, et al. Checkpoint blockade cancer immunotherapy targets tumour-specific mutant antigens. Nature. 2014; 515:577-581. doi:10.1038/nature13988.

37. Lin M, Luo H, Liang S, Chen J, Liu A, Niu L, et al. Pembrolizumab plus allogeneic nk cells in advanced non-small cell lung cancer patients. J Clin Invest. 2020; 130:2560-2569. doi:10.1172/jci132712.

38. Poznanski SM, Ritchie TM, Fan IY, El-Sayes A, Portillo AL, Ben-Avi R, et al. Expanded human nk cells from lung cancer patients sensitize patients' pdl1-negative tumors to pd1-blockade therapy. J Immunother Cancer. 2021; 9. doi:10.1136/jitc-2020-001933.

39. Dimopoulos MA, Dytfeld D, Grosicki S, Moreau P, Takezako N, Hori M, et al. Elotuzumab plus pomalidomide and dexamethasone for multiple myeloma. N Engl J Med. 2018; 379:1811-1822. doi:10.1056/NEJMoa1805762.

40. Gandhi UH, Cornell RF, Lakshman A, Gahvari ZJ, McGehee E, Jagosky MH, et al. Outcomes of patients with multiple myeloma refractory to cd38-targeted monoclonal antibody therapy. Leukemia. 2019; 33:2266-2275. doi:10.1038/s41375-019-0435-7.

41. Creelan BC, Antonia SJ. The nkg2a immune checkpoint - a new direction in cancer immunotherapy. Nat Rev Clin Oncol. 2019; 16:277-278. doi:10.1038/s41571-019-0182-8.

42. McWilliams EM, Mele JM, Cheney C, Timmerman EA, Fiazuddin F, Strattan EJ, et al. Therapeutic cd94/nkg2a blockade improves natural killer cell dysfunction in chronic lymphocytic leukemia. Oncoimmunology. 2016; 5:e1226720. doi:10.1080/2162402x.2016.1226720.

43. Borst $\mathrm{L}$, van der Burg SH, van Hall T. The nkg2a - hla-e axis as a novel checkpoint in the tumor microenvironment. Clin Cancer Res. 2020; 26:5549-5556. doi:10.1158/1078-0432.ccr-19-2095.

44. Gang M, Marin ND, Wong P, Neal CC, Marsala L, Foster M, et al. Car-modified memory-like nk cells exhibit potent responses to nk-resistant lymphomas. Blood. 2020; 136:2308-2318. doi:10.1182/blood.2020006619. 
45. Berrien-Elliott MM, Cashen AF, Cubitt CC, Neal CC, Wong P, Wagner JA, et al. Multidimensional analyses of donor memory-like nk cells reveal new associations with response after adoptive immunotherapy for leukemia. Cancer Discov. 2020; 10:1854-1871. doi:10.1158/2159-8290.cd-200312.

46. Sun JC, Lanier LL. Nk cell development, homeostasis and function: Parallels with cd8 ${ }^{+} \mathrm{t}$ cells. Nat Rev Immunol. 2011; 11:645-657. doi:10.1038/nri3044.

47. Jonsson AH, Yokoyama WM. Natural killer cell tolerance licensing and other mechanisms. Adv Immunol. 2009; 101:27-79. doi:10.1016/s0065-2776(08)01002-x.

48. Anfossi N, André P, Guia S, Falk CS, Roetynck S, Stewart CA, et al. Human nk cell education by inhibitory receptors for mhc class i. Immunity. 2006; 25:331-342. doi:10.1016/j.immuni.2006.06.013.

49. Kim S, Poursine-Laurent J, Truscott SM, Lybarger L, Song Y-J, Yang L, et al. Licensing of natural killer cells by host major histocompatibility complex class i molecules. Nature. 2005; 436:709-713. doi:10.1038/nature03847.

50. Orr MT, Lanier LL. Natural killer cell education and tolerance. Cell. 2010; 142:847-856. doi:https://doi.org/10.1016/j.cell.2010.08.031.

51. André P, Denis C, Soulas C, Bourbon-Caillet C, Lopez J, Arnoux T, et al. Anti-nkg2a mab is a checkpoint inhibitor that promotes anti-tumor immunity by unleashing both $t$ and nk cells. Cell. 2018; 175:1731-1743.e1713. doi:10.1016/j.cell.2018.10.014.

52. Kamiya T, Seow SV, Wong D, Robinson M, Campana D. Blocking expression of inhibitory receptor nkg2a overcomes tumor resistance to nk cells. J Clin Invest. 2019; 129:2094-2106. doi:10.1172/JCl123955.

53. Figueiredo C, Seltsam A, Blasczyk R. Permanent silencing of nkg2a expression for cell-based therapeutics. J Mol Med (Berl). 2009; 87:199-210. doi:10.1007/s00109-008-0417-0.

54. Stadtmauer EA, Fraietta JA, Davis MM, Cohen AD, Weber KL, Lancaster E, et al. Crispr-engineered $\mathrm{t}$ cells in patients with refractory cancer. Science. 2020; 367:eaba7365. doi:10.1126/science.aba7365.

55. van Loenen MM, de Boer R, Amir AL, Hagedoorn RS, Volbeda GL, Willemze R, et al. Mixed t cell receptor dimers harbor potentially harmful neoreactivity. Proc Natl Acad Sci U S A. 2010; 107:1097210977. doi:10.1073/pnas.1005802107.

56. Bendle GM, Linnemann C, Hooijkaas AI, Bies L, de Witte MA, Jorritsma A, et al. Lethal graft-versushost disease in mouse models of $t$ cell receptor gene therapy. Nat Med. 2010; 16:565-570, 561p following 570. doi:10.1038/nm.2128.

57. Hu W, Zi Z, Jin Y, Li G, Shao K, Cai Q, et al. Crispr/cas9-mediated pd-1 disruption enhances human mesothelin-targeted car t cell effector functions. Cancer Immunol Immunother. 2019; 68:365-377. doi:10.1007/s00262-018-2281-2.

58. Schubert ML, Schmitt M, Wang L, Ramos CA, Jordan K, Müller-Tidow C, et al. Side-effect management of chimeric antigen receptor (car) t-cell therapy. Ann Oncol. 2021; 32:34-48. doi:10.1016/j.annonc.2020.10.478.

59. Daher M, Basar R, Gokdemir E, Baran N, Uprety N, Nunez Cortes AK, et al. Targeting a cytokine checkpoint enhances the fitness of armored cord blood car-nk cells. Blood. 2021; 137:624-636. doi:10.1182/blood.2020007748.

60. Rautela J, Surgenor E, Huntington ND. Drug target validation in primary human natural killer cells using crispr rnp. J Leukoc Biol. 2020; 108:1397-1408. doi:10.1002/jlb.2ma0620-074r.

61. Morimoto T, Nakazawa T, Matsuda R, Nishimura F, Nakamura M, Yamada S, et al. Crispr-cas9mediated tim3 knockout in human natural killer cells enhances growth inhibitory effects on human glioma cells. Int J Mol Sci. 2021; 22. doi:10.3390/ijms22073489.

62. Guo X, Mahlakõiv T, Ye Q, Somanchi S, He S, Rana H, et al. Cblb ablation with crispr/cas9 enhances cytotoxicity of human placental stem cell-derived nk cells for cancer immunotherapy. J Immunother Cancer. 2021; 9:e001975. doi:10.1136/jitc-2020-001975.

63. Romee R, Foley B, Lenvik T, Wang Y, Zhang B, Ankarlo D, et al. Nk cell cd16 surface expression and function is regulated by a disintegrin and metalloprotease-17 (adam17). Blood. 2013; 121:35993608. doi:10.1182/blood-2012-04-425397.

64. Pomeroy EJ, Hunzeker JT, Kluesner MG, Lahr WS, Smeester BA, Crosby MR, et al. A genetically engineered primary human natural killer cell platform for cancer immunotherapy. Mol Ther. 2020; 28:52-63. doi:https://doi.org/10.1016/j.ymthe.2019.10.009.

65. Pillai K, Pourgholami MH, Chua TC, Morris DL. Muc1 as a potential target in anticancer therapies. Am J Clin Oncol. 2015; 38:108-118. doi:10.1097/COC.0b013e31828f5a07.

66. Kaur S, Kumar S, Momi N, Sasson AR, Batra SK. Mucins in pancreatic cancer and its microenvironment. Nat Rev Gastroenterol Hepatol. 2013; 10:607. doi:10.1038/nrgastro.2013.120. 
67. Martínez-Sáez N, Castro-López J, Valero-González J, Madariaga D, Compañón I, Somovilla VJ, et al. Deciphering the non-equivalence of serine and threonine o-glycosylation points: Implications for molecular recognition of the th antigen by an anti-muc1 antibody. Angew Chem Int Ed Engl. 2015; 54:9830-9834. doi:10.1002/anie.201502813.

68. Jing X, Liang H, Hao C, Yang X, Cui X. Overexpression of muc1 predicts poor prognosis in patients with breast cancer. Oncol Rep. 2019; 41:801-810. doi:10.3892/or.2018.6887.

69. Bhatia R, Gautam SK, Cannon A, Thompson C, Hall BR, Aithal A, et al. Cancer-associated mucins: Role in immune modulation and metastasis. Cancer Metastasis Rev. 2019; 38:223-236. doi:10.1007/s10555-018-09775-0.

70. Cloosen S, Gratama J, Van Leeuwen EBM, Senden-Gijsbers BLMG, Oving EBH, Von MensdorffPouilly S, et al. Cancer specific mucin-1 glycoforms are expressed on multiple myeloma. $\mathrm{Br} \mathrm{J}$ Haematol. 2006; 135:513-516. doi:10.1111/j.1365-2141.2006.06331.x.

71. Baldus SE, Wienand JR, Werner JP, Landsberg S, Drebber U, Hanisch FG, et al. Expression of muc1, muc2 and oligosaccharide epitopes in breast cancer: Prognostic significance of a sialylated muc1 epitope. Int J Oncol. 2005; 27:1289-1297

72. Van Elssen CH, Frings PW, Bot FJ, Van de Vijver KK, Huls MB, Meek B, et al. Expression of aberrantly glycosylated mucin-1 in ovarian cancer. Histopathology. 2010; 57:597-606. doi:10.1111/j.1365-2559.2010.03667.x.

73. Ibrahim NK, Yariz KO, Bondarenko I, Manikhas A, Semiglazov V, Alyasova A, et al. Randomized phase ii trial of letrozole plus anti-muc1 antibody as1402 in hormone receptor-positive locally advanced or metastatic breast cancer. Clin Cancer Res. 2011; 17:6822-6830. doi:10.1158/10780432.ccr-11-1151.

74. Braun DP, Crist KA, Shaheen F, Staren ED, Andrews S, Parker J. Aromatase inhibitors increase the sensitivity of human tumor cells to monocyte-mediated, antibody-dependent cellular cytotoxicity. Am J Surg. 2005; 190:570-571. doi:10.1016/j.amjsurg.2005.06.013.

75. Verheijen RH, Massuger LF, Benigno BB, Epenetos AA, Lopes A, Soper JT, et al. Phase iii trial of intraperitoneal therapy with yttrium-90-labeled hmfg1 murine monoclonal antibody in patients with epithelial ovarian cancer after a surgically defined complete remission. J Clin Oncol. 2006; 24:571578. doi:10.1200/jco.2005.02.5973.

76. Taylor-Papadimitriou J, Burchell JM, Graham R, Beatson R. Latest developments in muc1 immunotherapy. Biochem. Soc. Trans. 2018; 46:659-668. doi:10.1042/BST20170400.

77. Moreno M, Bontkes HJ, Scheper RJ, Kenemans P, Verheijen RHM, von Mensdorff-Pouilly S. High level of muc1 in serum of ovarian and breast cancer patients inhibits huhmfg-1 dependent cell-

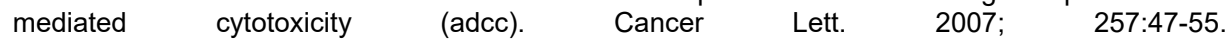
doi:https://doi.org/10.1016/j.canlet.2007.06.016.

78. Pericleous LM, Richards J, Epenetos AA, Courtenay-Luck N, Deonarain MP. Characterisation and internalisation of recombinant humanised hmfg-1 antibodies against muc1. Br J Cancer. 2005; 93:1257-1266. doi:10.1038/sj.bjc.6602847.

79. Madsen CB, Lavrsen K, Steentoft C, Vester-Christensen MB, Clausen H, Wandall HH, et al. Glycan elongation beyond the mucin associated tn antigen protects tumor cells from immune-mediated killing. PLoS One. 2013; 8:e72413. doi:10.1371/journal.pone.0072413.

80. Wandall HH, Blixt O, Tarp MA, Pedersen JW, Bennett EP, Mandel U, et al. Cancer biomarkers defined by autoantibody signatures to aberrant o-glycopeptide epitopes. Cancer Res. 2010; 70:1306-1313. doi:10.1158/0008-5472.can-09-2893.

81. Posey AD, Schwab RD, Boesteanu AC, Steentoft C, Mandel U, Engels B, et al. Engineered car $t$ cells targeting the cancer-associated tn-glycoform of the membrane mucin muc1 control adenocarcinoma. Immunity. 2016; 44:1444-1454. doi:10.1016/j.immuni.2016.05.014.

82. Smith SL, Kennedy PR, Stacey KB, Worboys JD, Yarwood A, Seo S, et al. Diversity of peripheral blood human nk cells identified by single-cell rna sequencing. Blood Adv. 2020; 4:1388-1406. doi:10.1182/bloodadvances.2019000699.

83. Filipovic I, Sönnerborg I, Strunz B, Friberg D, Cornillet M, Hertwig L, et al. 29-color flow cytometry: Unraveling human liver $\mathrm{nk}$ cell repertoire diversity. Front Immunol. 2019; 10:2692. doi:10.3389/fimmu.2019.02692.

84. Mahapatra S, Mace EM, Minard CG, Forbes LR, Vargas-Hernandez A, Duryea TK, et al. Highresolution phenotyping identifies nk cell subsets that distinguish healthy children from adults. PLoS One. 2017; 12:e0181134. doi:10.1371/journal.pone.0181134.

85. Horowitz A, Strauss-Albee DM, Leipold M, Kubo J, Nemat-Gorgani N, Dogan OC, et al. Genetic and environmental determinants of human nk cell diversity revealed by mass cytometry. Sci Transl Med. 2013; 5:208ra145. doi:10.1126/scitranslmed.3006702. 
86. Ni J, Wang X, Stojanovic A, Zhang Q, Wincher M, Bühler L, et al. Single-cell rna sequencing of tumor-infiltrating nk cells reveals that inhibition of transcription factor hif-1 $\alpha$ unleashes nk cell activity. Immunity. 2020; 52:1075-1087.e1078. doi:10.1016/j.immuni.2020.05.001.

87. Papalexi E, Satija R. Single-cell rna sequencing to explore immune cell heterogeneity. Nat Rev Immunol. 2018; 18:35-45. doi:10.1038/nri.2017.76.

88. Strauss-Albee DM, Fukuyama J, Liang EC, Yao Y, Jarrell JA, Drake AL, et al. Human nk cell repertoire diversity reflects immune experience and correlates with viral susceptibility. Sci Transl Med. 2015; 7:297ra115. doi:10.1126/scitranslmed.aac5722.

89. Herrera L, Santos S, Vesga MA, Anguita J, Martin-Ruiz I, Carrascosa T, et al. Adult peripheral blood and umbilical cord blood nk cells are good sources for effective car therapy against cd19 positive leukemic cells. Sci Rep. 2019; 9:18729. doi:10.1038/s41598-019-55239-y.

90. Woll PS, Grzywacz B, Tian X, Marcus RK, Knorr DA, Verneris MR, et al. Human embryonic stem cells differentiate into a homogeneous population of natural killer cells with potent in vivo antitumor activity. Blood. 2009; 113:6094-6101. doi:10.1182/blood-2008-06-165225.

91. Goldenson BH, Zhu H, Wang YM, Heragu N, Bernareggi D, Ruiz-Cisneros A, et al. Umbilical cord blood and ipsc-derived natural killer cells demonstrate key differences in cytotoxic activity and kir profiles. Front Immunol. 2020; 11:561553. doi:10.3389/fimmu.2020.561553.

92. Gong Y, Klein Wolterink RGJ, Wang J, Bos GMJ, Germeraad WTV. Chimeric antigen receptor natural killer (car-nk) cell design and engineering for cancer therapy. J Hematol Oncol. 2021; 14:73. doi:10.1186/s13045-021-01083-5.

93. Liu E, Marin D, Banerjee P, Macapinlac HA, Thompson P, Basar R, et al. Use of car-transduced natural killer cells in cd19-positive lymphoid tumors. N Engl J Med. 2020; 382:545-553. doi:10.1056/NEJMoa1910607.

94. Li Y, Hermanson DL, Moriarity BS, Kaufman DS. Human ipsc-derived natural killer cells engineered with chimeric antigen receptors enhance anti-tumor activity. Cell Stem Cell. 2018; 23:181-192. doi:10.1016/j.stem.2018.06.002.

95. Sarhan D, Brandt L, Felices M, Guldevall K, Lenvik T, Hinderlie P, et al. 161533 trike stimulates nkcell function to overcome myeloid-derived suppressor cells in mds. Blood Adv. 2018; 2:1459-1469. doi:10.1182/bloodadvances.2017012369.

96. Sheppard S, Sun JC. Virus-specific nk cell memory. J Exp Med. $2021 ; 218$. doi:10.1084/jem.20201731.

97. Cerwenka A, Lanier LL. Natural killer cell memory in infection, inflammation and cancer. Nat Rev Immunol. 2016; 16:112-123. doi:10.1038/nri.2015.9.

98. Finkelshtein D, Werman A, Novick D, Barak S, Rubinstein M. Ldl receptor and its family members serve as the cellular receptors for vesicular stomatitis virus. Proc Natl Acad Sci U S A. 2013; 110:7306-7311

99. Casasnovas JM. Virus-receptor interactions and receptor-mediated virus entry into host cells. Subcellular biochemistry. 2013; 68:441-466. doi:10.1007/978-94-007-6552-8_15.

100. Sutlu T, Nyström S, Gilljam M, Stellan B, Applequist SE, Alici E. Inhibition of intracellular antiviral defense mechanisms augments lentiviral transduction of human natural killer cells: Implications for gene therapy. Hum Gene Ther. 2012; 23:1090-1100. doi:10.1089/hum.2012.080.

101. Huijskens M, Walczak M, Sarkar S, Atrafi F, Gijsbers BLMG, Tilanus MGJ, et al. Ascorbic acid promotes proliferation of natural killer cell populations in culture systems applicable for natural killer cell therapy. Cytotherapy. 2015; 17:613-620. doi:10.1016/j.jcyt.2015.01.004.

102. Nanbakhsh A, Best B, Riese M, Rao S, Wang L, Medin J, et al. Dextran enhances the lentiviral transduction efficiency of murine and human primary nk cells. J Vis Exp. 2018; 131:55063. doi:10.3791/55063.

103. Heffner GC, Bonner M, Christiansen L, Pierciey FJ, Campbell D, Smurnyy Y, et al. Prostaglandin e2 increases lentiviral vector transduction efficiency of adult human hematopoietic stem and progenitor cells. Mol Ther. 2018; 26:320-328. doi:10.1016/j.ymthe.2017.09.025.

104. Radek C, Bernadin O, Drechsel K, Cordes N, Pfeifer R, Sträßer P, et al. Vectofusin-1 improves transduction of primary human cells with diverse retroviral and lentiviral pseudotypes, enabling robust, automated closed-system manufacturing. Hum Gene Ther. 2019; 30:1477-1493. doi:10.1089/hum.2019.157.

105. Raemer PC, Kohl K, Watzl C. Statins inhibit nk-cell cytotoxicity by interfering with Ifa-1-mediated conjugate formation. Eur J Immunol. 2009; 39:1456-1465. doi:10.1002/eji.200838863.

106. Tanaka T, Porter CM, Horvath-Arcidiacono JA, Bloom ET. Lipophilic statins suppress cytotoxicity by freshly isolated natural killer cells through modulation of granule exocytosis. Int Immunol. 2007; 19:163-173. doi:10.1093/intimm/dxl133. 
107. Hillyard DZ, Nutt CD, Thomson J, McDonald KJ, Wan RK, Cameron AJ, et al. Statins inhibit nk cell cytotoxicity by membrane raft depletion rather than inhibition of isoprenylation. Atherosclerosis. 2007; 191:319-325

108. Colamartino ABL, Lemieux W, Bifsha P, Nicoletti S, Chakravarti N, Sanz J, et al. Efficient and robust nk-cell transduction with baboon envelope pseudotyped lentivector. Front Immunol. 2019; 10:2873. doi:10.3389/fimmu.2019.02873.

109. Eyquem J, Mansilla-Soto J, Giavridis T, van der Stegen SJ, Hamieh M, Cunanan KM, et al. Targeting a car to the trac locus with crispr/cas9 enhances tumour rejection. Nature. 2017; 543:113-117. doi:10.1038/nature21405.

110. Howe SJ, Mansour MR, Schwarzwaelder K, Bartholomae C, Hubank M, Kempski H, et al. Insertional mutagenesis combined with acquired somatic mutations causes leukemogenesis following gene therapy of scid-x1 patients. J Clin Invest. 2008; 118:3143-3150. doi:10.1172/JCI35798.

111. Hacein-Bey-Abina S, Garrigue A, Wang GP, Soulier J, Lim A, Morillon E, et al. Insertional oncogenesis in 4 patients after retrovirus-mediated gene therapy of scid-x1. J Clin Invest. 2008; 118:3132-3142. doi:10.1172/JCI35700.

112. Gurney M, Stikvoort A, Nolan E, Kirkham-McCarthy L, Khoruzhenko S, Shivakumar R, et al. Cd38 knockout natural killer cells expressing an affinity optimized cd38 chimeric antigen receptor successfully target acute myeloid leukemia with reduced effector cell fratricide. Haematologica. 2020; Online ahead of print. doi:10.3324/haematol.2020.271908.

113. Sarkar RR, Gloude NJ, Schiff D, Murphy JD. Cost-effectiveness of chimeric antigen receptor t-cell therapy in pediatric relapsed/refractory b-cell acute lymphoblastic leukemia. J Natl Cancer Inst. 2019; 111:719-726. doi:10.1093/jnci/djy193.

114. Lin JK, Muffly LS, Spinner MA, Barnes JI, Owens DK, Goldhaber-Fiebert JD. Cost effectiveness of chimeric antigen receptor t-cell therapy in multiply relapsed or refractory adult large b-cell lymphoma. J Clin Oncol. 2019; 37:2105-2119. doi:10.1200/jco.18.02079.

115. Chicaybam L, Bonamino MH, Luckow Invitti A, Bortman Rozenchan P, de Luna Vieira I, Strauss BE. Overhauling car $t$ cells to improve efficacy, safety and cost. Cancers (Basel). 2020; 12. doi:10.3390/cancers12092360.

116. Thornton Snider J, Brauer M, Kee R, Batt K, Karaca-Mandic P, Zhang J, et al. The potential impact of car t-cell treatment delays on society. Am J Manag Care. 2019; 25:379-386 
202 | Chapter 6 


\section{Chapter 7}

Summary 
204 | Chapter 7 


\section{Summary}

In the present thesis, we continued our group's previous research on developing NK cell-based immunotherapy. In Chapter 1, we summarized the progress on tumor immunotherapy in general and introduced the NK cell-based immunotherapy.

In Chapter 2, we continued our group's previous work on the cancer-associated antigen MUC1. We hypothesized that defucosylation of a humanized murine anti-MUC1 antibody improved anti-cancer responses by enhancing NK cell responses. Therefore, we humanized the murine 5E5 anti-MUC1-Tn antibody and also generated an Fc-tail defucosylated antibody. We confirmed that the humanized $5 \mathrm{E} 5$ antibody recognizes MUC1-Tn epitopes and binds to the CD16 (FcyRllla) receptor on NK cells. The humanized 5E5 antibody could induce CD107a degranulation and triggered ADCC on NK cells when added to MUC1-Tn positive tumor cells. The Fc defucosylated antibody further boosted ADCC. However, we also found a dose-dependent reduction of CD16 expression levels on NK cells. MUC1-Tn antigens have been reported that could be dynamic internalized via endocytosis on cancer cells which could be an important mechanism for tumor escape and resistance. However, we found that adding endocytosis inhibitors during the cytotoxicity assays of NK cells against MUC1-Tn positive tumor cells, antibodies neither further augment CD107a degranulation nor improved ADCC of NK cells. In conclusion, the newly generated humanized anti-MUC1 antibodies improved anti-cancer responses using NK cells, especially after defucosylation of the Fc-tail.

Genetic editing aided by lentiviral transduction of human NK cells is still a major hurdle in NK cell-based immunotherapy. Therefore, in Chapter 3, we tried to increase the lentiviral transduction efficiency on human NK cells. We screened 5 statins and 5 non-statin compounds for their ability to increase VSV-G receptor-LDLR expression levels on NK cells. Only statins significantly upregulated LDLR levels, thereby increasing the transduction efficiency. We confirmed that the LDLR surface expression level correlated with the VSV-G lentivirus transduction efficiency. The statins-induced reduction of NK cell cytotoxicity could be completely restored using GGPP. Thus, we found that statins (especially rosuvastatin) plus GGPP can increase VSV-G lentivirus transduction efficiency on NK cells via upregulating LDLR. This is an important step in increasing the efficiency of genetic editing of NK cells for immunotherapy.

In Chapter 4, we provide a comprehensive review of the state-of-the-art of CAR-NK development with a focus on the NK cell-specific design of the CAR. We conducted statistical analysis of the various components that make up a CAR-NK from all published CAR-NK studies. From these statistics, we extracted best-practice and future ideas for the design of an optimal CAR for NK cells. In this chapter, we also detail our own progress on the generation of anti-MUC1-Tn-based CAR-NK cells (scFv from the 5E5 
antibody tested in chapter 2). These preliminary data provide insights in the technical challenges posed by the generation of CAR-NK cells.

In Chapter 5, we explored if genetic deletion of the inhibitory NKG2A receptor from human NK cells can improve NK cell function. We successfully generated NKG2Adeficient NK cells by using Cas9 Ribonucleoprotein (RNP) electroporation on expanded NK cells. Then, we analyzed the transcriptome, phenotype and functional aspects of the NKG2A knockout NK cells. We observed neither substantial alterations in gene expression levels by using RNA-seq and RT-PCR analyses, nor phenotypic or functional differences at baseline. Interestingly, NKG2A-knockout NK cells can fully overcome the inhibitory signals provided by HLA-E+ tumor cells. Importantly, genetic deletion of NKG2A constantly outperformed anti-NKG2A antibody blockage. Thus, we confirm that Cas9-based deletion of NKG2A is a powerful and efficient way to enhance anti-tumor responses in NK cells. We envisage that NKG2A-deficient NK cells will persist and perform better in the suppressive tumor microenvironment.

In Chapter 6, we summarized the findings from the various chapters. We provide an in-depth discussion of how these findings can be applied to the tumor immunology field to enhance NK cell-based therapies. Performing this work, we expect that we have added small building blocks to make CAR-NK cells more functional in the challenge to cure cancer. 


\section{Samenvatting}

In dit proefschrift zetten we het eerdere onderzoek van onze groep naar de ontwikkeling van NK-cel-immunotherapie voort. In hoofdstuk 1 hebben we de voortgang van tumorimmunotherapie samengevat en expliciet de op NK-cellen gebaseerde immunotherapie geïntroduceerd.

In Hoofdstuk 2 gaan we door met onderzoek dat onze groep aan kanker geassocieerd antigeen MUC1 heeft uitgevoerd. We hebben het uit muis afkomstige 5E5 anti-MUC1-Tn antilichaam gehumaniseerd en dit antilichaam van een gedefucosyleerde Fc-staart voorzien. Het gehumaniseerde 5E5-antilichaam herkende de MUC1-Tnepitopen en bond de CD16 (FcyRIIl $\alpha$ )-receptor op NK-cellen. Het gehumaniseerde 5E5antilichaam induceerde degranulatie van NK-cellen en veroorzaakte ook een Antilichaam afhankelijke cellulaire cytotoxiciteit (ADCC in het Engels) van NK-cellen na het samenbrengen met MUC1-Tn-positieve tumorcellen. Het Fc-gedefucosyleerde antilichaam versterkte de ADCC verder. Er is melding gemaakt van MUC1-Tn-antigenen die dynamisch kunnen worden geïnternaliseerd op kankercellen via endocytose, wat een belangrijk mechanisme zou kunnen zijn voor het ontsnappen van tumoren en resistentie. We ontdekten echter dat het toevoegen van endocytoseremmers tijdens de cytotoxiciteitstesten van NK-cellen tegen MUC1-Tn-positieve tumorcellen, antilichamen de CD107a-degranulatie niet verder versterken, noch ADCC van NK-cellen verbeteren. Concluderend verbeterden de nieuw gegenereerde gehumaniseerde anti-MUC1antilichamen de antikankerreacties met behulp van NK-cellen, vooral na defucosylering van de Fc-staart.

In Hoofdstuk 3 hebben we geprobeerd de transductie-efficiëntie van menselijke NKcellen met lentivirussen te verhogen. We hebben 5 statines en 5 niet-statine verbindingen gescreend om LDL Receptor op NK-cellen te verhogen omdat de lentivirale VSV-G envelope via de LDLR naar binnen gaat. Alleen de statines verhoogden de LDLR aanzienlijk, waardoor de transductie-efficiëntie werd verhoogd. We hebben waargenomen dat het LDLR-oppervlakte-expressieniveau correleerde met de VSV-Glentivirus transductie-efficiëntie. Van statines is beschreven dat ze de functie van NKcellen beperken, maar GGPP zou de onderdrukking volledig kunnen herstellen. We zijn dus van mening dat statines (vooral rosuvastatine) plus GGPP de transductie-efficiëntie van VSV-G-lentivirus op NK-cellen kunnen verhogen via het opreguleren van LDLR.

In Hoofdstuk 4 hebben we een uitgebreid literatuuroverzicht gemaakt van de stateof-the-art van ontwerpen en maken van CAR-NK cellen. We hebben statistiek uitgevoerd over het ontwerp van elk element van het CAR-construct uit bijna alle gepubliceerde CAR-NK-onderzoeken. Op basis van deze statistieken hopen we het ontwerp van een optimale CAR voor NK-cellen te extrapoleren en begeleiden. Daarnaast hebben we onze eigen voortgang van het genereren van anti-MUC1-Tn CAR-NK beschreven in boxes. Deze voorlopige gegevens moeten leiden tot gedetailleerde procedures om CAR-NK in de praktijk te genereren. 
In Hoofdstuk 5 hebben we het transcriptoom, het fenotype en de functionele aspecten van NKG2A knock-out NK-cellen onderzocht. We hebben met succes een NKG2A knock-out gegenereerd met behulp van CRISPR guideRNA en Cas9 Ribonucleoproteïne (RNP) elektroporatie op menselijke geëxpandeerde NK-cellen. We hebben geen substantiële verandering in genexpressie waargenomen na RNA-Seq deep sequencing en qPCR, als ook niet in de fenotypemarkers en functionele moleculen. Deze cellen zijn dus nauwelijks veranderd. Interessant is dat de NKG2A knock-out NKcel populatie de remming van tumorcellen met hoge HLA-E-expressie kunnen weerstaan, beter dan blokkade door een anti-NKG2A-antilichaam. We voorzien dat de NKG2A knock-out NK-cellen productie van NK-cellen voor klinische toepassing kan vergemakkelijken. Van deze cellen verwachten we dat ze persistenter zijn in de onderdrukkende tumormicro-omgeving.

In Hoofdstuk 6 hebben we een samenvatting van elk hoofdstuk en daarover een diepgaande discussie gemaakt. We hebben de belangrijkste bevindingen, beperkingen en toepassingen van elk hoofdstuk op een rij gezet. We hopen dat deze ontdekkingen de theorie van NK-cellen voor tumorimmunotherapie zullen verrijken. Door het uitvoeren van dit werk verwachten we dat we kleine bouwstenen hebben toegevoegd aan het domein van de brede kennis om CAR-NK-cellen functioneler te maken in de ambitie om kanker te genezen. 


\section{论文总结}

本论文中，继续本课题组前期有关人自然杀伤性 (NK) 细胞肿瘤免疫疗法的研究。在第 一章中，我们总结了最新的肿瘤免疫疗法研究进展，并详细介绍了 NK 细胞相关肿瘤免 疫治疗方法。

在第二章中，结合本研究团队之前对癌症相关抗原 MUC1-Tn 靶点的研究成果，我们假 设将抗 MUC1-Tn 抗体的 Fc 段修饰，去除岩藻糖亚基，能够增强 NK 细胞的抗体依赖的 细胞毒性杀伤 (ADCC) 。因此本章研究内容为将鼠来源的 5E5 靶向 MUC1-Tn 抗体序 列人源化，同时构建 $\mathrm{Fc}$ 段去岩藻糖基化的抗体。结果证明了人源化 $5 \mathrm{E} 5$ 抗体能够特异性 识别 MUC1-Tn 肿瘤抗原表位，并能够结合 NK 细胞上的 CD16(FcyRIIIa)分子。当加入 MUC1-Tn 抗原阳性的肿瘤细胞混合后，人源化 5E5 抗体可诱导 NK 细胞进行 CD107a 脱颗粒并介导 NK 细胞的 ADCC 杀伤肿瘤。有趣的是，人源化的 $5 E 5$ 靶向 MUC1-Tn 抗 体在 FC 段去除岩藻糖基化后，更进一步诱导 NK 细胞的 CD07a 脱颗粒和诱导 ADCC。 然而，当添加更多抗体剂量时，NK 细胞上表达的 CD16 分子表达会减少。相关研究已 报道，MUC1-Tn 抗原可以通过癌细胞内吞作用动态内化，这可能是肿瘤逃逸和抵抗的 重要机制。通过在 MUC1-Tn 阳性肿瘤细胞与 NK 细胞细胞毒性测定过程中，添加内吞 抑制剂不会进一步增强 NK 细胞的 CD107a 脱颗粒和 ADCC。因此，本课题组研发的人 源化抗 MUC1 抗体，促进 NK 细胞的抗肿瘤细胞毒性，抗体 FC 段去岩藻糖基化更进一 步促进其抗肿瘤效应。

对人 NK 细胞进行基因编辑仍然是 NK 细胞免疫疗法一个很大挑战。在第三章中，我们 旨在如何提高人类 NK 细胞的慢病毒转导效率。我们簃选了 5 种他汀类药物和 5 种非他 汀类化合物，用来诱导 NK 细胞上的慢病毒 VSV-G 衣壳蛋白受体—LDLR 分子。只有 他汀类化合物显着上调 NK 细胞表面的 LDLR 分子，从而有助于 VSV-G 病毒颗粒结合 NK 细胞，提高慢病毒转导效率。我们发现在 NK 细胞中， LDLR 表达水平与 VSV-G 慢 病毒转导效率正相关。但是前期有报道显示，他汀类药物会抑制 NK 细胞的功能，我们 发现 GGPP 化合物可以完全恢复他汀类对 NK 细胞的抑制作用。因此，我们相信他汀类 药物（尤其是瑞舒伐他汀）加 GGPP 可以通过上调 LDLR 病毒蛋白受体来提高 NK 细胞 
上的 VSV-G 慢病毒转导效率。本章实验结果，将有助于改善人体原代 NK 细胞基因编辑 效率，将促进 NK 细胞肿瘤免疫疗法。

在第四章中，我们详细介绍了 $N K$ 细胞免疫疗法的最新技术一一嵌合抗原受体 NK 细胞 (CAR-NK) 。本章节回顾了 CAR-NK 肿瘤细胞治疗技术的最新进展。我们对已发表的 CAR-NK 技术进行了统计，分析了 CAR-NK 设计每个作用元素的效果，这些设计的细节 能够预测和指导 CAR-NK 的效果。同时，本章节也详细展示了本课题组在建立针对 MUC1-Tn 抗原靶点的 CARNK 进展，这是在第二章人源化 5E5 抗体和第三章 NK 细胞 慢病毒转导的研究前期成果上进行的。这些初步数据，展示了构建 CAR-NK 细胞治疗技 术过程的各种挑战和技术难点。

在第五章中，我们探讨了 NKG2A 抑制性受体在 CRISPR/Cas9 技术进行基因敲除后，是 否能够促进 NK 细胞的杀伤肿瘤效应，同时 NKG2A 的敲除对人原代 NK 细胞的转录组、 表型和功能方面的影响。对体外扩增的原代 NK 细胞，采用 Cas9 核糖核蛋白 (RNP)+gRNA 电穿孔的方式，成功敲除了 NKG2A 抑制性受体。我们通过 RNA-seq 转 录组深度测序和定量 $\mathrm{qPCR}$ 监测基因表达水平的变化、以及流式细胞术进行细胞变性和 功能分子表达检测。结果显示，通过与未詀除 NKG2A 的 NK 细胞相比较，NKG2A 敲除 的 NK 细胞从基因表达转录组水平至细胞表型和杀伤功能分子的表达没有显著差异。有 趣的是，NKG2A 敲除的 NK 细胞，可以克服来自高 HLA-E 表达肿瘤细胞的抑制作用， 其阻断效率比抗 NKG2A 抗体更好。由此我们可以推测，NKG2A 受体敲除的 NK 细胞， 可以促进 NK 细胞克服 HLA-E 分子高表达的抑制性肿瘤微环境的限制。

在第六章中，我们对每一章进行了总结和深入讨论。我们列出了每一章的主要发现并讨 论了研究的局限性，并探讨了这些研究成果在临床肿瘤疾病治疗中的转化应用。我们希 望这些发现将丰富 NK 细胞，尤其是 CAR-NK，用于肿瘤免疫治疗的理论。 
Impact Paragraph | 211

\section{Impact Paragraph}



Cancer is a leading cause of death that threatens the life quality of human beings all over the world [1]. Traditional treatments have achieved great success in early diagnosed cancer, but have poor outcome in the advanced disease, especially in refractory and relapse stages where metastasis tumors exist [2,3]. Immunotherapy is an emerging therapy that aims to boost the immune system to eliminate the tumor cells and has been considered as the scientific breakthrough in 2013 [4]. NK cells are potent killer cells against cancer cells and can be further genetically modified to express a chimeric antigen receptor (CAR), which will be a powerful alternative effector cell for cancer therapy [5,6]. In the current thesis, we continue our group's previous experience and try to generate a natural killer (NK) cell-based immunotherapy for hematologic and solid tumors.

\section{Target group}

In advanced stages of cancer, morbidity and mortality are high due to cancer cells becoming insensitive to traditional treatments, such as chemotherapy and radiotherapy [7]. Of note, an estimated 19.3 million new cancer cases and almost 10 million cancer deaths occurred in 2020 [1]. These patients are in need of an effective therapy to control the progress of their disease and alleviation of suffering. Immunotherapy was recently introduced as a new treatment modality that also shows promising results in advanced disease [8]. CAR-NK cells are an example of immunotherapy and have gained interest due to their effectiveness and favourable safety profile. Although the current CAR-NK may only be effective in some hematologic malignancies, there are also some effects on solid tumors reported. Combination therapy with cytokine and checkpoint blockade antibodies may further improve the outcome of NK cell-based therapy.

\section{Innovation and implementation}

Throughout my PhD project, we aimed to develop CAR-NK and CRISPR/Cas9 genetically modified NK cells as an advanced therapeutic medicinal product (ATMP) for cancer treatment. We attempted to generate a flexible strategy for making CAR-NK cells and also to systematically test additional mechanisms to enhance NK cell mediated anticancer effects via antibodies or CRISPR/Cas9 technology. These generated optimization strategies can be extended further than our model diseases, breast cancer and multiple myeloma (MM), as we expect that the prerequisites for effective signalling are universal for NK cells. We have successfully expanded human NK cells within a GMP facility that can be used in clinical trials for solid tumor patients, rendering a much cheaper ATMP (Chapter 2 4) compared to the current CAR-T cells (see below). Moreover, we also applied CRISPR/Cas9 to introduce genetic modifications, thereby disrupting the NKG2A inhibitory receptor on NK cells to boost the persistence and efficiency of killing tumor cells in an immunosuppressive tumor microenvironment (TME) (Chapter 5). Combined with the extensive immunotherapy experience in our laboratory, we expect that this knowledge will be easily adaptable and applicable for the development of other anti-tumor strategies in the coming future.

Even though CAR-engineered lymphocytes are currently only studied in the context of cancer, they can also be used to control diseases, such as infections and auto- 
immune disorders. Given the rapid increase in antibiotic resistance, the development of alternative strategies is highly relevant. As NK cells have the intrinsic capacity to recognize and eliminate virus-infected cells (such as SARS-CoV-2), their responses might be further enhanced by equipping them with a CAR [9]. Such cells could be applied in patients in the intensive care units, outbalancing the cost for long stay at the ICU ward. Till May 2021, there are five CAR-T ATMP products approved by the U.S. Food and Drug Administration (FDA) for hematologic malignancy, while there are currently no CAR-T cells registered as effective therapy for solid tumors. Moreover, in some CAR-T infused patients, several severe side-effects have been reported, including a cytokine storm that may even be lethal. Here, NK cells have several advantages over T cells. Firstly, NK cells are strong killer cells to cancerous target cells which can be generated in a GMP-compliant large-scale expansion in only 2 weeks. Secondly, NK cells lack the T cell receptor (TCR), which allows for the use of allogeneic NK cells, resulting in an "offthe-shelf" product. The TCR on T cells are the major molecule involved severe graft versus host disease (GVHD), which will not occur in case of NK cells. Moreover, several clinical trials have demonstrated that infusion of allogeneic NK cells to both hematologic and solid tumors are safe and feasible, and even induces graft-versus-leukaemia cytotoxicity [10-12]. This favourable profile of NK cells broadens the applicability of NK cells in clinical trials and daily clinical practice.

\section{Sociall and economic relevance}

With increasing numbers of patients being treated with ATMP and achieving encouraging outcomes, we can expect that more ATMP cellular products will be approved by the FDA or European Medicine Agency (EMA). The policies related to the ATMP will lift limitations on genetic modified cellular products to guarantee that more cancer patients can get appropriate and safe immunotherapy. These policies will also encourage companies to take part in the development of ATMPs. Together, this will lead to positive effects on the whole society by improving the health and welfare of all human beings.

Besides the encouraging social effects of these ATMPs, the economical aspect needs to be considered. One of the big hurdles of the distribution of these ATMPs is the economic burden [13]. The cost of the two FDA-approved anti-CD19 CAR-T cell therapy products are $\$ 475,000$ (Tisagenlecleucel; Novartis, Basel, Switzerland) and $\$ 373,000$ (Axicabtagene Ciloleucel; Gilead/Kite Pharma, Foster City, CA, USA) per patient, with an estimated amount of $\$ 1$ million per patient when costs with medical tests and hospitalization are included [14]. There are several studies conducted to evaluate the cost-effectiveness of the CAR-T in diffuse large B cells lymphoma (DLBL) and some models were made to predict how to reduce the cost $[15,16]$. If the price remains at this level, many patients will not be able to afford this therapy, especially in countries with a weaker economic status. As mentioned before, CAR-NK is an alternative effector cell source that most likely will be cheaper than CAR-T therapy.

Another approach for reducing the price of immunotherapy is to decrease the cost associated with CAR-NK production [14,17], such as using a non-viral transfection method to replace the current costly lentiviral approach, and produce the "off-the-shelf" CAR-NK products. The already mentioned severe cytokines release storms (CRS) that have often been reported in CAR-T infused patients, will also give an additional economic burden. The first Phase I/II CD19 CAR-NK expanded from cord blood 
elucidated that these cells are safe and effective for B cell lymphoma patients without severe side effects that have been reported in CAR-T therapy [12]. Thus, CAR-NK may further diminish the costs of ATMP products. Of note, one study proposed that decentralized ATMP production would allow even further cost reductions due to easier logistics and reduced cell preservation fees [18]. With the development of ATMP production technology, more people, or societies in general, ultimately can afford CARNK therapy.

Our group has investigated NK cells for more than 20 years and has developed several products for malignant disease. Our group leaders, Prof. Dr G.M.J. Bos and Dr. W.T.V. Germeraad, have established the start-up company CiMaas that transfers these novel cellular therapies to clinical application. CiMaas has the protocols, procedures and equipment in a B-grade clean room that that allows to produce GMP grade, expanded NK cells to clinical applicable numbers of 3E10 within 2 weeks. The infrastructure established by CiMaas together with Maastricht University Medical Center (MUMC+) will also be capable of testing CAR-NK cells in clinical trials in the future. Following the model of CiMaas, we believe more and more hospitals and institutes will transform and implement novel immunotherapies in clinical trials and will become a reality in cancer treatment. 


\section{References}

1. Sung H, Ferlay J, Siegel RL, Laversanne M, Soerjomataram I, Jemal A, et al. Global cancer statistics 2020: Globocan estimates of incidence and mortality worldwide for 36 cancers in 185 countries. CA Cancer J Clin. 2021. doi:10.3322/caac.21660.

2. Waldman AD, Fritz JM, Lenardo MJ. A guide to cancer immunotherapy: From t cell basic science to clinical practice. Nat Rev Immunol. 2020; 20:651-668. doi:10.1038/s41577-020-0306-5.

3. $\mathrm{Xu} \mathrm{X,} \mathrm{Li} \mathrm{T,} \mathrm{Shen} \mathrm{S,} \mathrm{Wang} \mathrm{J,} \mathrm{Abdou} \mathrm{P,} \mathrm{Gu} \mathrm{Z,} \mathrm{et} \mathrm{al.} \mathrm{Advances} \mathrm{in} \mathrm{engineering} \mathrm{cells} \mathrm{for} \mathrm{cancer}$ immunotherapy. Theranostics. 2019; 9:7889-7905. doi:10.7150/thno.38583.

4. Galluzzi L, Vacchelli E, Bravo-San Pedro JM, Buqué A, Senovilla L, Baracco EE, et al. Classification of current anticancer immunotherapies. Oncotarget. 2014; 5:12472-12508. doi:10.18632/oncotarget.2998.

5. June $\mathrm{CH}$, Sadelain M. Chimeric antigen receptor therapy. N Engl J Med. 2018; 379:64-73. doi:10.1056/NEJMra1706169.

6. Dunbar CE, High KA, Joung JK, Kohn DB, Ozawa K, Sadelain M. Gene therapy comes of age. Science. 2018; 359:eaan4672. doi:10.1126/science.aan4672.

7. Myers JA, Miller JS. Exploring the nk cell platform for cancer immunotherapy. Nat Rev Clin Oncol. 2020. doi:10.1038/s41571-020-0426-7.

8. Miller JS, Lanier LL. Natural killer cells in cancer immunotherapy. Annu Rev Cancer Biol. 2019; 3:77103. doi:10.1146/annurev-cancerbio-030518-055653.

9. Ma M, Badeti S, Chen $\mathrm{CH}$, Pinter A, Jiang Q, Shi L, et al. Car-nk cells effectively target the d614 and g614 sars-cov-2-infected cells. bioRxiv. 2021. doi:10.1101/2021.01.14.426742.

10. Khatua S, Cooper LJN, Sandberg DI, Ketonen L, Johnson JM, Rytting ME, et al. Phase i study of intraventricular infusions of autologous ex vivo expanded nk cells in children with recurrent medulloblastoma and ependymoma. Neuro Oncol. 2020; 22:1214-1225. doi:10.1093/neuonc/noaa047.

11. Ciurea SO, Schafer JR, Bassett R, Denman CJ, Cao K, Willis D, et al. Phase 1 clinical trial using mbil21 ex vivo-expanded donor-derived nk cells after haploidentical transplantation. Blood. 2017; 130:1857-1868. doi:10.1182/blood-2017-05-785659.

12. Liu E, Marin D, Banerjee P, Macapinlac HA, Thompson P, Basar R, et al. Use of car-transduced natural killer cells in cd19-positive lymphoid tumors. N Engl J Med. 2020; 382:545-553. doi:10.1056/NEJMoa1910607.

13. Lyman GH, Nguyen A, Snyder S, Gitlin M, Chung KC. Economic evaluation of chimeric antigen receptor t-cell therapy by site of care among patients with relapsed or refractory large b-cell lymphoma. JAMA Netw Open. 2020; 3:e202072. doi:10.1001/jamanetworkopen.2020.2072.

14. Chicaybam L, Bonamino MH, Luckow Invitti A, Bortman Rozenchan P, de Luna Vieira I, Strauss BE. Overhauling car $\mathrm{t}$ cells to improve efficacy, safety and cost. Cancers (Basel). 2020; 12. doi:10.3390/cancers12092360.

15. Thielen FW, van Dongen-Leunis A, Arons AMM, Ladestein JR, Hoogerbrugge PM, Uyl-de Groot CA. Cost-effectiveness of anti-cd19 chimeric antigen receptor t-cell therapy in pediatric relapsed/refractory b-cell acute lymphoblastic leukemia. A societal view. Eur J Haematol. 2020; 105:203-215. doi:10.1111/ejh.13427.

16. Lin JK, Muffly LS, Spinner MA, Barnes JI, Owens DK, Goldhaber-Fiebert JD. Cost effectiveness of chimeric antigen receptor t-cell therapy in multiply relapsed or refractory adult large b-cell lymphoma. J Clin Oncol. 2019; 37:2105-2119. doi:10.1200/jco.18.02079.

17. Couchoud C, Fagnoni P, Aubin F, Westeel V, Maurina T, Thiery-Vuillemin A, et al. Economic evaluations of cancer immunotherapy: A systematic review and quality evaluation. Cancer Immunol Immunother. 2020; 69:1947-1958. doi:10.1007/s00262-020-02646-0.

18. Ran T, Eichmüller SB, Schmidt $P$, Schlander M. Cost of decentralized car t-cell production in an academic nonprofit setting. Int J Cancer. 2020; 147:3438-3445. doi:10.1002/ijc.33156. 


\section{Acknowledgements}


I would like to express my sincere gratitude to my supervision team. Prof. Gerard M.J. Bos, dear Gerard, thank you so much for give this opportunity to conduct my PhD in this great group. Thanks for offer some furnitures when I arrived in Maastricht. Thank you for helping me with my CSC scholarship application. I still remember that once I would like stop the CAR-NK experiment and you encouraged me to continue and brought me to the international NK meeting in Luxemburg 2019. In the NK meeting, I was inspired by the great work from the presentations and posters and we had a detailed discussion on the plans for feasible CAR-NK in our lab. In November, we got the opportunity to visit the experted CAR-T/CAR-NK group of Prof Jianxiang Wang and Prof Min Wang in Institute of Hematology and Blood Diseases Hospital in Tianjin, P.R. China. After that, we visited the Palace Museum in the Forbidden City and the Great Wall in Beijing. There is a saying in Chinese that one who fails to reach the Great Wall is not a hero (real man). Thanks for gave me such a good experience and memory. In my last year of My PhD, thanks for your kindness support to extend 1 year for my PhD. I am very appreciated the questions you challenged during our Tuesday's group meeting and each projects, which are very important aspects to leading me critical thinking. Thanks for invite me to your home for playing table tennis, hiking on the Sint-Pietersberg and having dinner. Thanks for showing me all the perfect qualities that make you a wonderful boss. I feel very grateful for having had the opportunity to study in our group and having had you as my promotor. Thank you for your unfailing guidance and support throughout my years of PhD. Dr. Wilfred T.V. Germeraad, dear Wilfred, thank you for your fantastic supervising on my PhD. I am so lucky and proud to be your PhD student. Thank you for helping me with my CSC scholarship application. Thank you for your patient with me. You guided me, step by step, at the beginning of my study. I could discuss any problems with you that I met from every projects. I still remember the day when you pick me at the Maastricht central station and offer me some furnitures to live in September 2016. I also remember that you drive together with me to Prof. Winfried Wels' group in Franfurt, Germany to learn CAR-NK technology in September 2018. We had a nice Chinese cuisine there and a nice talk about the plans for my projects. I have learned so much from you. Thank you very much for your tremendous efforts to revise the published manustript and my PhD thesis. You always have a unique and sharp comments that are very crucial for the professional field of tumor immunotherapy. You are always there 
whenever I need you. Indeed, you are my 'Super Supervisor'! Dr. Roel G.J. Klein Wolterink, dear Roel, thank you for your intensive and professional supervision on my PhD. I am so lucky and proud to be your PhD student. Thank you for helping me with my CSC scholarship application. Your views on tumor immunotherapy and academic research have greatly enlightened me. Your continuous encouragement and recognition of my work have been a great inspiration to me. Thanks for inviting me to your home and Chinese restaurant for dinner. Although you continuned your Postdoc in Lisbon, the every Wednesday meeting always let us be connected. Thank you for your constant support and all your valuable feedback on my work. When encountering challenges, I always tend to make them more difficult, and you are always the one to help me to make them easier. You are always there whenever I need you. I have learned so much from you. Thank you for helping me to become a better person inside and out. Thank you to my supervisor team, we will keep in touch in the future and I will explore the possibility to further collaboration to allow me further learn from you.

I appreciate Dr. Lotte Wieten from department of Transplantation Immunology, Maastricht University Medical Center. Dear Lotte, thank you for your valuable comments and feedback on my presentations and manuscript. I still remember that you together with Wilfred and Gerard interviewed me at the first time. Thank you for bring me to the conferences (DHC and NK meetings) and we had nice talk during the journey. I learned a lot from you from the Basic Immunology class that you organized. Thanks for your kind words and encouragement to me, which have great impact on me. I am especially grateful to Dr. Arjan J. Groot from MAASTRO Lab for teaching me how to improve the lentiviral production and human primary cells transduction. Dear Arjan, I learned a lot from the discussion with you on some technical problems, such as CRISPR/cas 9 and molecular cloning. Your answers always help me a lot. Thanks for your encouraging on me. Thanks for MAASTRO Lab, Maastricht Radiation Oncology offer the equipments to allow me doing molecular cloning and plasmid purification.

I would like to thank my PhD defense paranymphs. Dear Nicky, You are always very friendly and helpful. Thank you for your help and encouragement. You are very smart and doing well with your projects and I do believe you will have a good outcome for your $\mathrm{PhD}$ and bright future. Good luck with your PhD and all your projects. Dear Antonia, you are very cheerful and hard work. Please be always keep smiling and being optimistic. 
You are clever and good at communication. It is a nice memory to chat with you during the coffee break and lunch time. Good luck with your $\mathrm{PhD}$ and all your projects.

I would like express my sincerely appreciation to my dear colleagues: Dr. Janine C.H.M.J Van Elssen, dear Janine, thank you for your help especially the valuable comments from you during my progress reports in the group meetings. You work careful and very hard. Good luck with your works and projects. Birgit, thanks for your wonderful organize the lab and the FACS machine. Thanks for set-up the protocol of K562-mIL21-4-1BBL feeder cell based human primay NK cell expansion protocol. Thank you very much for your kindness help during my PhD. Thanks for your kind words and encouragement to me. Eric-Jan, thanks for your help in set-up the F012 feeder cell based NK cell expansion in the lab. You have a good sense of humurous that everybody work with you are feel happy and comfortable. You are very professional in organizing and conducting experiments. I wish you all the best in the future. Femke, thank you for your help during my PhD. You are very smart, confident, and always helpful. You are good at planning and organizing the experiments. I learned a lot from you. I wish you all the best for your PhD and your future. Lynn, Thank you very much for your help during my PhD. You are familiar a lot of important experiment skills. You are eager to learn and always helpful to others. Sabine, Thank you for your kindness help to me. You work very hard and work enthusiasticly in the lab. It is a nice memory to chat with you during the coffee break and lunch time. You are very professional in organizing and conducting experiments. I wish you all the best in the future. Niken, thank you for your help and encouragement for me. Thanks for inviting me to join your wedding ceremony in Rotterdam. I wish you all the best with your family. Gwendolyn, thank you for your help and encouragement to me. I wish all the best to your work and PhD. Marijn, Thanks for invite me to your house. It is a nice memory with your cats. Thank you for your help during my PhD. Timo, thanks for arranging the Sanger sequencing and discussing the experiment set-up. I wish you all the best for your work and future. Fausto, thank you for taking care of my sanger sequencing samples. That help me a lot. My thanks are extended to all my other colleagues and friends: Robert, Burcu, Michel, Silvie, Lera, Kedir, Ian, Ahmed, Luuk, Maggee, Vicky, Maria and Jossie... Thanks the help from Secretaresse Hematologie Esther Houben for arranging the meeting with my supervisors and sending out my $\mathrm{PhD}$ thesis. Thank you for helping me out make appointment with all the oppsition professors and experts. Thanks the help from 
Managing Director of GROW Judith Doomen-Dormans and the Office Manager of GROW Brigitte Custers. I deeply appreciate the time spent with all of you, and thanks to all of you for giving me such a beautiful memory, one that I will cherish for a lifetime. I still remember the moment when I got the opportunity to fulfill my PhD dream in Prof. Gerard Bos group at Maastricht University. I was enthusiastically introuduced by Prof. Harry Steinbusch, we met in Beijing 2015 CSC workshop. I was so excited to start a new journey in research, and was curious about the life study abroad. Simultaneously, I was worried about the challenges that studying and living in a foreign country at that time. Now, I have been in Maastricht for five years, and I am about to complete my PhD. Looking back, I have enjoyed the life in Maastricht very much, which is a nice memory of my life. At the end of my PhD thesis, I want to express my sincerely appreciations to my dear supervisors, colleagues, friends, and family. This accomplishment would not have been possible without you. Thank you.

First, I would like to thank the funding from the China Scholarship Council (CSC: 201707720056) to support my PhD study. I am appreciated for the recommendation and assistance in applying the CSC funding from Prof. Harry Steinbusch and Mr. Wang Zhen.

I would also like to thank the members of my thesis evaluation committee: Prof. dr. Dirk De Ruysscher (Chairman), Prof. dr. Jürgen Kuball, Prof. dr. Tuna Mutis, Prof. dr. Erik Beckers, and Dr. Ludwig Dubois. Thank you for your effort and time to evaluate my thesis and thank you for your valuable comments and suggestions.

I would like to thank you the international collaborative team. Thank you Prof. Dr. Winfried Wels for granting me to learn CAR-NK technology from your group. Thanks for Dr CongCong Zhang's and other kind colleagues' help. Thanks for offer me the 2 weeks accomodation in the Georg-Speyer-Haus, Institute for Tumor Biology and Experimental Therapy, Frankfurt am Main, Germany. We thank Prof. dr. Reno Debets and Dr Cor Hj Lamers (Erasmus University Medical Center - Daniel den Hoed Cancer Center, Rotterdam, The Netherlands.) kindly provide the CAIX CAR retrovirus supernatant. 感谢天津血液病研究所的王建祥教授和王敏教授给予我和我导师参观您们 的实验室，让我有机会向您们课题组学习，期待未来有机会向您们进一步学习。 
I am also grateful to my Chinese colleagues and friends in Maastricht University. 在此特别感谢荷兰马斯特里赫特大学的各位老师、学长和同学的帮助：张明师姐和何彦 兵师兄，感谢您们对我的帮助，祝愿您们生活和工作一切顺利! 万俊和王珊珊，感谢俊 哥夫妇的款待和帮助，祝愿你们继续深造期间学业有成，工作顺利！丁世杰和刘美超， 感谢丁哥在马城的照顾和帮助，祝愿丁哥的“人造肉”事业大展鸿图! 李正文，感谢李 哥在马城对我的照顾和帮助，祝你工作顺利！感谢郭乐的周末热情款待和专业的学术交 流，祝你工作顺利！感谢武思宇的学术交流与分享和帮助，祝你学业有成，博士毕业顺 利! 感谢罗倩的帮助，感谢罗博士的学术交流和款待，祝你一切顺利！感谢王珊的帮助， 很佩服你能平衡工作和生活，你的摄影技术和厨艺都很棒，祝你在马城学习快乐！感谢 黄盛世的款待和周末一起锻炼身体，祝你学业有成，博士毕业顺利! 感谢罗江幸和列永 婵的帮助，感谢鸿幸积极组织周末篮球友谊赛，很佩服你们，祝你们在马城一切顺利， 工作顺利，期待广州见！感谢曾海燕的帮助和款待，祝愿你读博一切顺利！感谢邓敏的 帮助和学术交流，祝愿你读博和工作一切顺利！感谢间秋婷的款待、学术交流和帮助， 祝你学业有成，博士毕业顺利！感谢张幸真和邹今幂的款待和周末一起锻炼身体，祝你 学业有成，工作顺利，博士毕业顺利! 感谢孙思雨的款待和帮助，祝你学业有成，博士 毕业顺利！感谢王锐师兄、王萍老师、马老师、郭老师、战老师、李总、刘红、谭秀香、 石镇维、严承功、张帅、余艺文、刘豫、丁伶伶、陈琳、桑亚秋、殷冬梅、刘晓松、徐 盼师姐、辛葍师姐、姚天宇师姐、宗盛华师兄和徐青师姐、忝奇、安宁师姐、张硕师兄、 王振老师、李雯婕、李林峰、张笑迪、靳顺鍂、冯正师兄、刘丹、王朝、Sunny 老 师。。。非常幸运能够认识您们，感恩能够与您们一起在荷兰马斯特里赫打篮球、骑行、 爬山、吃饭、交流和学习，使得生活变得有趣很多! 感谢有您们的教海和帮助。非常感 谢沈倩姐和刘彦超学长的关照，祝您们工作顺利! 
感谢河北省唐山市华北理工大学医学院基础医学院李琳老师、裴银辉教授的教导，特别 感谢李老师的栽培，老师您的谆谆教诲时刻索绕在我的脑海中，令我一生受用！感谢唐 山市开滦集团总医院安新老师、张军老师、高利常老师的指导。感谢河南省郑州大学第 一附属医院苏会芳博士的帮助和鼓励。感谢广东省广州市中山大学中山医学院的吴长有 教授、李亮平教授的教导和栽培。感谢广州市红十字会医院吴文法博士、周涛龙医生的 帮助与支持。感谢中山大学中山眼科中心的曹乾忠博士的帮助。特别感谢江西省安远县 孔田镇黄晓龙和黄美龙一家人的大力帮助与支持! 特别感谢魏先生一家人的大力帮助与 支持！感谢各位老师们、长辈们、学长们和同学们的教导与帮助，我铭记于心! 感谢我的女朋友张棚琳的关心和支持，感谢你一直不离不弃，不断地鼓励我、开导我、 激发我的潜能，感谢你的陪伴，疫情原因，我们虽然有二十多个月未相见，但是彼此仍 能够相互鼓励相互促进，我一直都在，愿望往后余生，请你多多指教。

Finally, I must express my very profound gratitude to my family. 亲爱的爸爸妈妈， 感谢您们的鼓励、教导和无条件的支持，让我有机会去超越自己，让我更进一步认识自 己。感谢姐姐与姐夫和哥哥与大嫂的关心和支持，你们是我的好榜样。感谢大姑一家人、 大叔一家人、二叔一家人和二姑一家人等长辈们及各位家人们的教导和关心，让我感受 到大家庭的温暖和关怀。感谢外公外婆的挂念和鼓励，感谢舅舅们和姨妈们的支持与鼓 励。家人们的关心和问候，是我前行的强大动力，感谢您们! 感谢自己足够坚强和勇敢，二十余载的求学生涯，尚未有读完万卷书，却行了万里路， 带着满腔的热血，盼望回到我热爱的祖国，以梦为马，砥砺前行，未来可期，愿自己能 够为祖国卫生健康事业做出贡献! 
224 | Acknowledgements 


\section{Curriculum vitae}


Ying Gong was born on July 6th, 1989 in Ganzhou, Jiangxi Province, P.R. China. In 2007, he was admitted to Hebei United University and started studying in medical examination. In 2012, he obtained his Medical Bachelor's degree, after which he started studying his master of Medicine at Sun Yat-sen University. He obtained Medical Master's degree in 2015. In 2016, Ying was interviewed with Prof. Harry Steinbusch and was introduced to Prof. Gerard Bos' group in the department of Internal Medicine of the Maastricht University. Ying obtained his Chinese medical practitioner's Qualification Certificate in 2018. He was awarded a national grant from the Chinese Scholarship Council (CSC), which sponsored his PhD study. During his PhD, Ying researched on human natural killer cell-based immunotherapy for cancer immunotherapy under the supervision of Prof. Gerard M.J. Bos, Dr. Wilfred T.V. Germeraad and Dr. Roel G.J. Klein Wolterink. The results of his research are presented in current thesis. 


\section{Scientific output}




\section{List of publications}

- Gong Y, Klein Wolterink RGJ, Janssen I, Groot AJ, Bos GMJ, Germeraad WTV. Rosuvastatin enhances VSV-G lentiviral transduction of NK cells via upregulation of the low-density lipoprotein receptor. Mol Ther Methods Clin Dev. 2020; 17:634-646. doi:10.1016/j.omtm.2020.03.017.

- Gong Y, Klein Wolterink RGJ, Wang J, Bos GMJ, Germeraad WTV. Chimeric antigen receptor natural killer (CAR-NK) cell design and engineering for cancer therapy. J Hematol Oncol. 2021; 14:73. doi:10.1186/s13045-021-01083-5.

- Gong Y, Klein Wolterink RGJ, Gulaia V, Cloosen S, Ehlers FAI, Wieten L, Graus YF, Bos GMJ, Germeraad WTV. Defucosylation of tumor-specific humanized anti-MUC1 monoclonal antibody enhances NK cell-mediated anti-tumor cell cytotoxicity. Cancers. 2021; 13:2579. doi:10.3390/cancers13112579.

- Gong Y, Germeraad WTV, Janssen L, Gijsbers BLMG, Olieslagers TI, Bos GMJ, Klein Wolterink RGJ. NKG2A genetic deletion promotes human NK cell antitumor responses by reversing HLA-E-mediated inhibition (Submitted to Frontiers in Immunology).

\section{Oral presentations}

- 13th Dutch Hematology Congress 2019 (Arnhem, The Netherlands): MUC1CAR-NK therapy for cancer immunotherapy.

\section{Poster presentations}

- Maastricht University GROW school science day 2017 (Maastricht, The Netherlands): MUC1-CAR-NK therapy for cancer immunotherapy.

- Maastricht University GROW school science day 2020 (Maastricht, The Netherlands): Enhancing the killing capacity of NK cells by disrupting the inhibitory signal via CRISPR Cas9 technology (Best Poster Award). 